\title{
Raman-Spektroskopie kleiner Moleküle und Molekülaggregate im Überschallstrahl nach thermischer Anregung
}

\section{DisSERTATION}

zur Erlangung des mathematisch-naturwissenschaftlichen Doktorgrades „Doctor rerum naturalium“ der Georg-August-Universität Göttingen

$$
\text { im Promotionsprogramm Chemie }
$$

der Georg-August University School of Science (GAUSS)

vorgelegt von

Katharina Otto

aus Göttingen

Göttingen 2015 


\section{Betreuungsausschuss}

Prof. Dr. Martin A. Suhm

Georg-August-Universität Göttingen, Institut für Physikalische Chemie Jun.-Prof. Dr. Ricardo A. Mata

Georg-August-Universität Göttingen, Institut für Physikalische Chemie

\section{Mitglieder der Prüfungskommission}

Referent: Prof. Dr. Martin A. Suhm

Georg-August-Universität Göttingen, Institut für Physikalische Chemie Korreferent: Jun.-Prof. Dr. Ricardo A. Mata

Georg-August-Universität Göttingen, Institut für Physikalische Chemie

\section{Weitere Mitglieder der Prüfungskommission}

Prof. Dr. Ulf Diederichsen

Georg-August-Universität Göttingen, Institut für Organische und Biomolekulare Chemie Prof. Dr. Burkhard Geil

Georg-August-Universität Göttingen, Institut für Physikalische Chemie Prof. Dr. Andreas Janshoff

Georg-August-Universität Göttingen, Institut für Physikalische Chemie PD Dr. Thomas Zeuch

Georg-August-Universität Göttingen, Institut für Physikalische Chemie 
Zwei Dinge sind zu unserer Arbeit nötig: Unermüdliche Ausdauer und die Bereitschaft, etwas, in das man viel Zeit und Arbeit gesteckt hat, wieder wegzuwerfen.

Albert Einstein 

An erster Stelle möchte ich mich bei Prof. Dr. Martin A. Suhm für die exzellente Betreuung bedanken. Seine Diskussionsbereitschaft, Unterstützung und Begeisterungsfähigkeit haben entscheidend zum Gelingen dieser Arbeit beigetragen.

Bei Jun.-Prof. Dr. Ricardo A. Mata möchte ich mich für die Übernahme der Zweitbetreuung sowie die Beantwortung von Fragen zu quantenchemischen Rechnungen bedanken.

Dr. Nils Lüttschwager danke ich für die Einarbeitung in die heizbare Version der CurryJet Apparatur.

Für hilfreiche Diskussionen und für durch fachliche und nicht-fachliche Gespräche geprägte Kaffeepausen danke ich Dr. Julia Zischang, Matthias Heger, Thomas Forsting und Franz Kollipost. Für Unterstützung, wenn ich mal mehr als zwei Hände und ein Augenpaar brauchte, danke ich besonders meinem Labornachbar Jonas Altnöder und außerdem meinem Nachfolger Sebastian Bocklitz. Außerdem danke ich Jonas Altnöder, Sebastian Bocklitz, Thomas Forsting, Matthias Heger und Dr. Julia Zischang sowie Friederike Steensen für das Korrekturlesen von Teilen dieser Arbeit.

Bei der gesamten Arbeitsgruppe Suhm möchte ich mich für die angenehme Arbeitsatmosphäre bedanken, die über manch „Murphy-geprägten“ Arbeitstag hinweghalf.

Stellvertretend für die Werkstätten möchte ich mich bei Volker Meyer, Andreas Knorr und Annika von Roden bedanken. Ohne die Expertise der Werkstattmitarbeiter ist ein experimentelles Arbeiten kaum möglich. Besonders bei der Planung und Konstruktion der neuen Vakuumkammer und sämtlichen Hilfsmitteln, die für die Einkopplung des IRLasers notwendig waren, konnte ich mich immer auf den Ideenreichtum der Werkstätten hinsichtlich der praktischen Umsetzung meiner Wünsche verlassen.

Für finanzielle Unterstützung danke ich der DFG, die durch die Bewilligung des Antrags Su-121/2-2 das Arbeiten mit einem OPO-Laser erst ermöglicht hat, und außerdem dem Deutschen Akademischen Austauschdienst (DAAD) für die Ermöglichung einer Tagungsreise nach Belgien.

Nicht zuletzt möchte ich mich bei meiner Familie und meinen Freunden für ihre Unterstützung und den unerschütterlichen Glauben an meine Fähigkeiten bedanken. 



\section{Inhaltsverzeichnis}

1 Einleitung 1

2 Grundlagen 3

2.1 Lineare Raman-Spektroskopie im Überschallstrahl . . . . . . . . . . . . 3

2.2 Intensität von Raman-Übergängen . . . . . . . . . . . . . . . . . 4

2.2.1 Schwingungsübergänge . . . . . . . . . . . . . 5

2.2 .2 Rotationsübergänge . . . . . . . . . . . . . . . . . . 6

2.2.3 Depolarisation . . . . . . . . . . . . . . . . . . . 7

2.3 Temperaturbestimmung . . . . . . . . . . . . . . . 8

2.4 Davydov-Aufspaltung in symmetrischen Clustern . . . . . . . . . . 9

2.4 Trimer . . . . . . . . . . . . . . . . . 10

2.4 Tetramer............................... 11

2.4 .3 Pentamer . . . . . . . . . . . . . . . . . . 11

2.5 Optisch parametrischer Oszillator . . . . . . . . . . . . . . 12

3 Experimentelles 14

3.1 Curry-Jet Apparatur - Allgemeines . . . . . . . . . . . . . . . . . . 14

3.2 Eigenschaften der neuen Edelstahlexpansionskammer . . . . . . . . . . 17

3.3 Lineare Raman-Spektroskopie gekoppelt mit IR-Laseranregung . . . . . 20

3.3.1 OPO-Laser: Eigenschaften laut Hersteller . . . . . . . . . . . . . 21

3.3.2 OPO-Laser: Testmessungen . . . . . . . . . . . . . . 23

3.3.3 Unterdrückung der OPO-Strahlung zwischen 400 und $700 \mathrm{~nm} \quad$. 29

3.3.4 Idlerwellenlängenbestimmung mittels FTIR-Spektrometer . . . . 29

3.3.5 Einkopplung des OPO-Lasers in die Vakuumkammer und Justie-

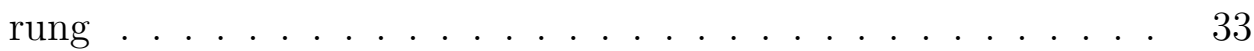

3.4 Spektrenauswertung und -abbildung . . . . . . . . . . . . . 37

4 Temperaturbestimmung in der Jetexpansion 39

4.1 Rotationstemperaturen . . . . . . . . . . . . . . . . . 40

4.1.1 Testsystem Stickstoff . . . . . . . . . . . . . . . . . . . . . 40

4.1.2 Referenzmessung Luft . . . . . . . . . . . . . . . . . 51

4.1.3 Rotationstemperatur von Wasser im Jet . . . . . . . . . . . . 54

4.2 Schwingungstemperaturen am Beispiel von 2,2,2-Trifluorethanol . . . . 57

4.3 Zusammenfassung und Ausblick . . . . . . . . . . . . . . . 61 
5 Raman-Spektroskopie kleiner Wasseraggregate 62

5.1 Einleitung . . . . . . . . . . . . . . . . . . 62

5.2 Jetexperimente im OH-Streckschwingungsbereich . . . . . . . . . . 64

5.2 .1 Tetramer........................... 68

5.2 .2 Trimer . . . . . . . . . . . . . . . . 70

5.2 .3 Pentamer ........................... 71

5.2.4 Weitere Clusterbanden . . . . . . . . . . . . . 75

5.3 OH-Knickschwingungen und OH-Streckschwingungsobertöne . . . . . . 76

5.4 Zusammenfassung und Ausblick . . . . . . . . . . . . . . . 78

6 Systeme zur Untersuchung von Isomerisierungen $\quad 80$

$6.12,2,2$-Trifluorethanol . . . . . . . . . . . . . . . . . 80

$6.2 \quad 3,3,3$-Trifluorpropanol . . . . . . . . . . . . . . . . . . . 88

$6.3 \quad N$-Methylformamid . . . . . . . . . . . . . . . . . . . . . . . . . . 93

6.4 Zusammenfassung und Ausblick . . . . . . . . . . . . . . . 101

7 IR-Laseranregung am Beispiel des Methanol-Dimers 102

7.1 Abschätzung des zu erwartenden Effektes auf das Raman-Spektrum . . 103

7.2 Testmessungen unter Nutzung der Paraboldüse . . . . . . . . . . . . . . 105

7.3 Testmessungen unter Nutzung der Zylinderlinse . . . . . . . . . . . . . 108

7.4 Diskussion und Ausblick . . . . . . . . . . . . . . . . . . . . . . 112

8 Zusammenfassung und Ausblick 116

A Anhang I

A.1 Publikationen ...................... I

A.2 Chemikalienliste . . . . . . . . . . . . . . I

A.3 Spektrenverzeichnisse . . . . . . . . . . . . . . . . . . II

A.4 Experimentelles . . . . . . . . . . . . . . . . . VII

A.4.1 Ergänzende Abbildungen . . . . . . . . . . . . . . . V VII

A.4.2 Experimentelle Parameter für die Aufnahme von FTIR-Spektren zur Bestimmung der Wellenzahl des OPO-Lasers . . . . . . . . . XII

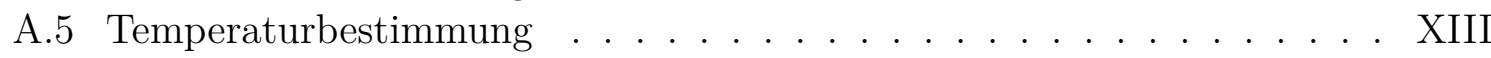

A.6 Wassercluster . . . . . . . . . . . . . . . . . XXI

A.7 Isomerisierungen . . . . . . . . . . . . . . . . XXIII

A.7.1 2,2,2-Trifluorethanol . . . . . . . . . . . . . . XXIII

A.7.2 3,3,3-Trifluorpropanol . . . . . . . . . . . . . XXIV

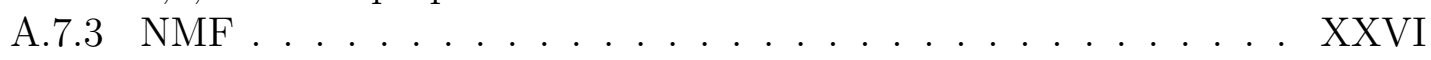

A.8 Methanol . . . . . . . . . . . . . . . . . . XXXX

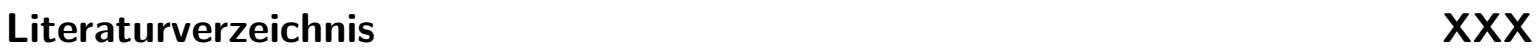




\section{Einleitung}

Zum Verständnis der Wechselwirkungen in kondensierten Systemen ist die Untersuchung kleiner Molekülaggregate eine gängige Methode um einen schrittweisen Übergang zwischen Gasphase und kondensierter Materie zu simulieren. ${ }^{1}$ Die Schwingungsspektroskopie ist ein wirkungsvolles Mittel zur Untersuchung von intermolekularen Wechselwirkungen. Besonders bei Wasserstoffbrücken-gebundenen Aggregaten ist die Technik wegen der systematischen Rotverschiebung der X-H-Streckschwingung mit der Stärke der Wasserstoffbrückenbindung sowie der Verstärkung der Intensität im IR-Spektrum gut geeignet. ${ }^{1}$ Die Kopplung dieser Spektroskopietechnik mit Überschallexpansionen bietet die Möglichkeit kleine Aggregate isoliert zu untersuchen. ${ }^{2}$ Neben den häufig genutzten IR-Techniken ${ }^{3,4}$ ist seit Mitte der 1970er Jahre auch die Raman-Spektroskopie genutzt und weiterentwickelt worden. ${ }^{5-7}$ In Göttingen ist sie seit 2006 etabliert $^{8}$ und mittlerweile hinsichtlich der Nachweisempfindlichkeit ${ }^{9,10}$ auch für schwerflüchtige Substanzen $^{11}$ verbessert worden. Sie liefert besonders für symmetrische Aggregate komplementäre Informationen zur IR-Spektroskopie. ${ }^{12}$ Außerdem ist sie für die Untersuchung von Monomeren, die im IR nur sehr schwache Signalstärken aufweisen, geeignet.

Als Weiterentwicklung der Methode sollte in der vorliegenden Arbeit eine Kopplung mit IR-Laseranregung erfolgen. Die Auswirkungen der Anregung können sich in verschiedenen Effekten niederschlagen. Neben der Erwärmung der Moleküle und Molekülaggregate sind auch Isomerisierungs- und für die Aggregate Dissoziationsreaktionen möglich. Weiterhin kann es zu einer intramolekularen Schwingungsumverteilung kommen (IVR), deren Auswirkungen im Spektrum sichtbar sind. ${ }^{13}$

Um diese verschiedenen Effekte eindeutig separieren zu können, ist es wichtig im Vorfeld verschiedene Methoden zur Interpretation von Spektren, die ohne eine IRLaseranregung aufgenommen wurden, zu entwickeln. Dementsprechend wurde in der vorliegenden Arbeit die bislang in der Jetspektroskopie wenig genutzte Methode zur Temperaturbestimmung unter Nutzung der Stokes- und Anti-Stokes-Streuintensitäten ${ }^{14,15}$ evaluiert. Außerdem wurde eine Kopplungsanalyse ${ }^{12}$ am Beispiel kleiner isolierter Wasseraggregate vorgenommen. ${ }^{15}$ Durch diese Analyse ist bereits ohne IRLaseranregung eine Aussage über die Geschwindigkeit der Energieumverteilung zwischen den miteinander gekoppelten, gebundenen $\mathrm{OH}-$ Oszillatoren möglich.

Zur Untersuchung von Isomerisierungsreaktionen ist es nützlich zunächst die Konformerenverteilung zu analysieren. ${ }^{16}$ Aus Raman-Jetspektren können unter bestimmten 
Voraussetzungen Energieunterschiede zwischen verschiedenen Konformeren ermittelt werden. ${ }^{17,18}$ In der vorliegenden Arbeit wurden die isomerisierungsdynamisch sehr unterschiedlichen Systeme 3,3,3-Trifluorpropanol und $N$-Methylformamid untersucht.

Nach einem kurzen Überblick über die Grundlagen folgt in Kapitel 3 die Beschreibung der bisherigen und der weiterentwickelten Apparatur. Für die Kopplung der beiden Anregungsmethoden wurde die verwendete Apparatur mit einer neuen Vakuumkammer ausgestattet. Außerdem wurde ein Dauerstrich-OPO-Laser beschafft, dessen Eigenschaften in umfangreichen Tests untersucht wurden. In Kapitel 4 werden dann zwei verschiedene Temperaturbestimmungsmethoden, die in der Raman-Jetspektroskopie genutzt werden können, vorgestellt und verglichen. Anschließend erfolgt in Kapitel 5 die Beschreibung der Experimente zu kleinen Wasseraggregaten, die IR-spektrokopisch im Jet bereits gut untersucht sind. ${ }^{4,19-22}$ Durch die Aufnahme von Raman-Spektren unter Nutzung einer heizbaren Düse wurde eine robuste Zuordnung der Banden bis zum Pentamer und unter Zuhilfenahme der vorhandenen IR-Daten die Analyse der Excitonenkopplung der gebundenen OH-Oszillatoren erst möglich. ${ }^{15}$ Danach werden in Kapitel 6 die Untersuchungen zu Isomerisierungsreaktionen im Jet erläutert. ${ }^{18}$ Erste Experimente zu Studien der Dissoziation mittels IR-Laseranregung am Beispiel des gut spektroskopisch untersuchten Methanoldimers ${ }^{12,23-25}$ werden in Kapitel 7 beschrieben. Abschließend folgen eine kurze Zusammenfassung sowie ein Ausblick auf weitere geplante Untersuchungen. 


\section{Grundlagen}

\subsection{Lineare Raman-Spektroskopie im Überschallstrahl}

Lineare Raman-Spektroskopie gekoppelt mit Überschallexpansionen ist seit 2006 in Göttingen etabliert. ${ }^{8}$ Die Technik nutzt den Raman-Effekt, der als inelastische Streuung von Photonen an Molekülen beschrieben werden kann. In erster Näherung ist die Streueffizienz proportional zur Änderung der Polarisierbarkeit $\alpha$ des Moleküls während der Molekülbewegung. Die Polarisierbarkeit selbst muss für anisotrop polarisierbare Moleküle als Tensor zweiter Stufe beschrieben werden. Üblicherweise werden die Invarianten $a$ und $\gamma$ des Tensors zur Beschreibung verwendet, dabei ist $a$ die mittlere Polarisierbarkeit und $\gamma$ die symmetrische Anisotropie. ${ }^{26}$ Der Hauptvorteil der Technik gegenüber nichtlinearen Raman-Verfahren liegt in der universellen Anwendbarkeit, so sind die Voraussetzungen für die Raman-Aktivität für Molekülrotationen eine anisotrope Polarisierbarkeit und für Molekülschwingungen die Änderung der Polarisierbarkeit mit der Schwingung. ${ }^{8}$ Insbesondere für inversionssymmetrische Moleküle und Molekülaggregate kann die Raman-Spektroskopie komplementäre Informationen liefern, die mittels IR-Techniken aufgrund des Alternativverbots nicht zugänglich sind. Ein Nachteil der Technik ist die geringe Effizienz des Streuprozesses. Dieser Umstand kann aber mittlerweile durch den Einsatz leistungsstarker Laser als Lichtquellen und empfindlicher Detektoren kompensiert werden.

Die Kopplung mit einer Überschallexpansion liefert ein leistungsstarkes Werkzeug zur Untersuchung isolierter Moleküle und Molekülkomplexe. Im Folgenden sollen einige Eigenschaften der Überschallexpansion erläutert werden. Genauere theoretische Betrachtungen können in Ref. 2,27-30 nachgelesen werden.

Bei einer Überschallexpansion wird ein Gas oder Gasgemisch von einem Reservoir mit dem Druck $p_{0}$ und der Temperatur $T_{0}$ durch eine kleine Öffnung in eine Kammer niedrigen Restdrucks $p_{\mathrm{b}}$ expandiert. Für eine freie Überschallexpansion muss der Durchmesser der Düse $D$ sehr viel größer als die mittlere freie Weglänge der Moleküle $\lambda_{0}$ sein, außerdem muss die Druckdifferenz zwischen Reservoir und Kammer ausreichend groß sein. ${ }^{2}$

Im Anfangsstadium erfolgt eine nahezu isentrope Expansion und die inneren Freiheitsgrade der Moleküle sind im Gleichgewicht. Durch eine hohe Anzahl an Stößen erfolgt 
einerseits die „Monochromatisierung“ der Geschwindigkeiten, die zu einer engen Geschwindigkeitsverteilung und somit zu einer starken Abkühlung führt, ${ }^{28}$ andererseits werden durch Stöße mindestens dreier Teilchen Molekülaggregate gebildet. ${ }^{29}$ Entlang der Expansion findet ein starker Abfall der Dichte statt, so dass ab einem bestimmten Punkt die Anzahl der Stöße nicht mehr ausreicht, um die Gleichgewichtsbedingungen der einzelnen Freiheitsgrade aufrecht zu erhalten. ${ }^{28}$ Die effizienteste Relaxation erfolgt für die Translationsfreiheitsgrade, daher können hier die Gleichgewichtsbedingungen am längsten aufrecht erhalten werden. Sobald das auch für die Translation nicht mehr möglich ist, endet die Abkühlung. Für die Endtemperaturen gilt $T_{\text {vib }}>T_{\text {rot }}>T_{\text {trans. }}{ }^{27}$ In diesem Bereich befindet sich die „Ruhezone“ der Expansion, in der die nun sehr stark abgekühlten Moleküle und Molekülaggregate in nahezu kollisionsloser Umgebung untersucht werden können. ${ }^{27}$

Die erreichbaren Endtemperaturen hängen hierbei maßgeblich vom Trägergas ab. In der vorliegenden Arbeit wurde mit He, Ar sowie Mischungen aus He mit Ne und He mit Ar gearbeitet. Stöße mit Trägergasatomen mit höherer Masse führen zu einer effizienteren Relaxation. Allerdings wird durch ein schwereres Trägergas auch die Clusterbildung begünstigt, was zu einer Erwärmung des Trägergases führt, da die Bindungsenergie der Aggregate abgeführt werden muss. ${ }^{29}$ Außerdem können die leichter polarisierbaren größeren Edelgasatome in höherem Ausmaß Induktions- und Dispersionswechselwirkungen mit den zu untersuchenden Molekülen eingehen, so dass es mitunter zu einer Kondensation des Trägergases auf einem Molekül(aggregat) kommen kann. ${ }^{16}$ Wenn unterschiedliche Konformere vorliegen, deren Energiebarrieren $>400 \mathrm{~cm}^{-1}$ sind, kann durch eine Expansion mit einem geeigneten Trägergas (meist Helium) das Konformerenverhältnis, das im thermischen Gleichgewicht vorlag, in einem frühen Stadium der Expansion eingefroren werden. ${ }^{31}$ Unter dieser Annahme können aus den Intensitätsunterschieden der zu verschiedenen Konformeren gehörigen Banden Rückschlüsse auf die Energieunterschiede zwischen den Konformeren gezogen werden. ${ }^{17}$

\subsection{Intensität von Raman-Übergängen}

Im folgenden Kapitel sollen die Intensitäten von Schwingungs- und Rotations-RamanÜbergängen diskutiert werden. Es soll dabei nur ein kurzer Überblick erfolgen; detaillierte Herleitungen finden sich in Ref. 8,9,26,32,33.

Die Intensität $I$ eines Raman-Übergangs folgt der allgemeinen Formel:

$$
I=C \cdot N_{\mathrm{k}}^{\mathrm{i}} \cdot \widetilde{\nu}_{\mathrm{s}}^{4} \cdot \Phi\left(a_{\mathrm{k}}^{2}, \gamma_{\mathrm{k}}^{2}, \theta\right) \cdot \mathcal{I}_{0}
$$

Hierbei ist $C$ eine Konstante, $N_{\mathrm{k}}^{\mathrm{i}}$ die Besetzung des Ausgangsniveaus $i$ und $\widetilde{\nu}_{\mathrm{s}}$ die Wellenzahl der Streustrahlung mit $\widetilde{\nu}_{\mathrm{s}}=\widetilde{\nu}_{0}-\widetilde{\nu}_{\mathrm{k}}$ für den k-ten Stokes-Übergang und $\widetilde{\nu}_{\mathrm{s}}=\widetilde{\nu}_{0}+\widetilde{\nu}_{\mathrm{k}}$ für den k-ten Anti-Stokes-Übergang. $\widetilde{\nu}_{0}$ ist die Wellenzahl und $\mathcal{I}_{0}$ die Irra- 
dianz des eingestrahlten Lichts. $\Phi$ beschreibt die Abhängigkeit der Intensität von den Invarianten des Polarisierbarkeitstensors $a_{\mathrm{k}}$ und $\gamma_{\mathrm{k}}$ (mit $a_{\mathrm{k}}$ der mittleren Polarisierbarkeit und $\gamma_{\mathrm{k}}$ der symmetrischen Anisotropie) sowie von der Streugeometrie ausgedrückt durch den Beobachtungswinkel $\theta \cdot{ }^{26} \Phi$ beinhaltet auch die Ableitungen der Invarianten nach den entsprechenden Schwingungskoordinaten.

\subsubsection{Schwingungsübergänge}

Zur Betrachtung von Schwingungen wird hier die doppelt harmonische Näherung angewendet. Im Rahmen der Näherung fallen die Schwingungswellenzahlen der Fundamentalen und aller heißen Banden zusammen. Durch Anharmonizitätseffekte können die Schwingungswellenzahlen der heißen Banden im Experiment auch abweichen. Bei Betrachtung der Streustrahlung, die von mehr als einem Molekül erzeugt wird, muss daher für die Intensität des Übergangs nicht nur die Besetzung des Grundzustands, sondern auch die Population höherer Niveaus sowie deren Übergangsmomente einbezogen werden.

Der gewichtete Besetzungsfaktor ${ }^{26} N_{\mathrm{k}}^{\mathrm{i}}$ ergibt sich aus der Summe der Beiträge der Fundamentale sowie aller heißen Banden.

Für Stokes-Übergänge ${ }^{26}$ ergibt sich somit

$$
N_{\mathrm{k}}^{\mathrm{S}}=N \cdot g_{\mathrm{k}} \cdot \sum_{i}\left(v_{\mathrm{k}}^{\mathrm{i}}+1\right) \frac{\exp \left\{-\left(v_{\mathrm{k}}^{\mathrm{i}}+1 / 2\right) h c_{0} \widetilde{\nu}_{k} / k T\right\}}{\sum \exp \left\{-\left(v_{\mathrm{k}}^{\mathrm{i}}+1 / 2\right) h c_{0} \widetilde{\nu}_{k} / k T\right\}}=\frac{N \cdot g_{\mathrm{k}}}{1-\exp \left\{-h c_{0} \widetilde{\nu}_{\mathrm{k}} / k T\right\}}
$$

und für Anti-Stokes-Übergänge ${ }^{26}$

$$
N_{\mathrm{k}}^{\mathrm{AS}}=\frac{N \cdot g_{\mathrm{k}}}{\exp \left\{h c_{0} \widetilde{\nu}_{\mathrm{k}} / k T\right\}-1}
$$

Mit $N$ der Gesamtzahl der Moleküle, $g_{\mathrm{k}}$ dem Entartungsfaktor der Schwingung, $\widetilde{\nu}_{\mathrm{k}}$ der Schwingungswellenzahl, $h$ der Planck-Konstante, $c_{0}$ der Vakuumlichtgeschwindigkeit, $k$ der Boltzmann-Konstante, $T$ der absoluten Temperatur und $v_{\mathrm{k}}^{\mathrm{i}}$ der Schwingungsquantenzahl des Ausgangsniveaus. Da die Gesamtzahl der Moleküle $N$ im Experiment nicht bekannt ist, können nur relative Intensitäten betrachtet werden.

Für die Gesamtintensität ergibt sich für senkrecht zur Streuebene polarisiert eingestrahltes Licht $\left(\perp^{i}\right)$, eine Streugeometrie von $90^{\circ}(\theta=\pi / 2)$ und die Erfassung sowohl senkrecht als auch parallel zur Streuebene polarisierter Streustrahlung $\left(\perp^{s}+\|^{s}\right)$ für Stokes Übergänge: ${ }^{26}$

$$
I^{\mathrm{S}}\left(\pi / 2, \perp^{s}+\|^{s}, \perp^{i}\right)=\frac{2 \pi^{2} h}{45 c_{0} \widetilde{\nu}_{\mathrm{k}}} \cdot \frac{\left(\widetilde{\nu}_{0}-\widetilde{\nu}_{\mathrm{k}}\right)^{4}}{1-\exp \left\{-h c_{0} \widetilde{\nu}_{\mathrm{k}} / k T\right\}} \cdot g_{\mathrm{k}} \cdot\left(45 a_{\mathrm{k}}^{\prime 2}+7 \gamma_{\mathrm{k}}^{\prime 2}\right) \cdot \mathcal{I}_{0}
$$


Und für Anti-Stokes-Übergänge ${ }^{26}$

$$
I^{\mathrm{AS}}\left(\pi / 2, \perp^{s}+\|^{s}, \perp^{i}\right)=\frac{2 \pi^{2} h}{45 c_{0} \widetilde{\nu}_{\mathrm{k}}} \cdot \frac{\left(\widetilde{\nu}_{0}+\widetilde{\nu}_{\mathrm{k}}\right)^{4}}{\exp \left\{h c_{0} \widetilde{\nu}_{\mathrm{k}} / k T\right\}-1} \cdot g_{\mathrm{k}} \cdot\left(45 a_{\mathrm{k}}^{2}+7 \gamma_{\mathrm{k}}^{\prime 2}\right) \cdot \mathcal{I}_{0}
$$

Hierbei sind $a_{\mathrm{k}}^{\prime}$ und $\gamma_{\mathrm{k}}^{\prime}$ die abgeleiteten Invarianten nach der Schwingungskoordinate $Q_{\mathrm{k}}$ und beschreiben somit die Änderung der Invarianten mit der k-ten Schwingung. Diese werden auch aus quantenchemischen Rechnungen mithilfe der Programme Gaussian $09^{34}$ und TURBOMOLE v6. $5^{35}$ erhalten. Im Falle von Gaussian 09 wird eine Streuaktivität $A_{\text {Streu }}$ ausgegeben. Sie ist folgendermaßen mit den Invarianten verknüpft:

$$
A_{\text {Streu }}=45 a_{\mathrm{k}}^{\prime 2}+7 \gamma_{\mathrm{k}}^{\prime 2}
$$

Zum Vergleich der Intensitäten aus Rechnung und Experiment wird der differentielle Streuquerschnitt $\sigma^{\prime}=I / \mathcal{I}_{0}$ verwendet. Da im Experiment keine Energiedichte gemessen, sondern die Anzahl der Photonen bestimmt wird, muss die Formel dementsprechend angepasst werden (siehe Ref. 9). Somit ergibt sich für den differentiellen Streuquerschnitt für Stokes-Schwingungsübergänge: ${ }^{9,26}$

$$
\sigma^{\prime}\left(\pi / 2, \perp^{s}+\|^{s}, \perp^{i}\right)=\frac{2 \pi^{2} h}{45 c_{0} \widetilde{\nu}_{\mathrm{k}}} \cdot \frac{\left(\widetilde{\nu}_{0}-\widetilde{\nu}_{\mathrm{k}}\right)^{3} \cdot \widetilde{\nu}_{0}}{1-\exp \left\{-h c_{0} \widetilde{\nu}_{\mathrm{k}} / k T\right\}} \cdot g_{\mathrm{k}} \cdot\left(45 a_{\mathrm{k}}^{\prime 2}+7 \gamma_{\mathrm{k}}^{\prime 2}\right)
$$

und entsprechend für Anti-Stokes-Schwingungsübergänge:

$$
\sigma^{\prime}\left(\pi / 2, \perp^{s}+\|^{s}, \perp^{i}\right)=\frac{2 \pi^{2} h}{45 c_{0} \widetilde{\nu}_{\mathrm{k}}} \cdot \frac{\left(\widetilde{\nu}_{0}+\widetilde{\nu}_{\mathrm{k}}\right)^{3} \cdot \widetilde{\nu}_{0}}{\exp \left\{h c_{0} \widetilde{\nu}_{\mathrm{k}} / k T\right\}-1} \cdot g_{\mathrm{k}} \cdot\left(45 a_{\mathrm{k}}^{\prime 2}+7{\gamma_{\mathrm{k}}^{\prime}}^{2}\right)
$$

\subsubsection{Rotationsübergänge}

Für reine Rotationsübergänge sollen hier nur zweiatomige Moleküle in der Näherung des starren Rotators betrachtet werden.

Für die Besetzung gilt: ${ }^{26,36,37}$

$N_{\mathrm{k}}=N \cdot g_{\mathrm{N}} \cdot\left(2 J_{\mathrm{k}}+1\right) \frac{\exp \left\{-E\left(J_{\mathrm{k}}\right) / k T\right\}}{Z_{\mathrm{rot}}}=N \cdot g_{\mathrm{N}} \cdot\left(2 J_{\mathrm{k}}+1\right) \frac{\exp \left\{-B \cdot J_{\mathrm{k}}\left(J_{\mathrm{k}}+1\right) h c_{0} / k T\right\}}{Z_{\mathrm{rot}}}$

Wobei $g_{\mathrm{N}}$ der Entartungsfaktor aus der Kernspinstatistik, $B$ die Rotationskonstante, $J_{\mathrm{k}}$ die Rotationsquantenzahl des Ausgangsniveaus und $Z_{\text {rot }}$ die Rotationszustandssumme ist. Als Gesamtintensität ergibt sich für Stokes-Übergänge: ${ }^{26}$

$$
\begin{aligned}
& I^{\mathrm{S}}\left(\pi / 2, \perp^{s}+\|^{s}, \perp^{i}\right)= \\
& \frac{\pi^{2}}{6 \epsilon_{0}^{2}} \cdot g_{\mathrm{N}} \cdot N\left(\widetilde{\nu}_{0}-\widetilde{\nu}_{\mathrm{k}}\right)^{4} \frac{\exp \left\{-B \cdot J_{\mathrm{k}}\left(J_{\mathrm{k}}+1\right) h c_{0} / k T\right\}}{Z_{\mathrm{rot}}} \frac{\left(J_{\mathrm{k}}+1\right)\left(J_{\mathrm{k}}+2\right)}{\left(2 J_{\mathrm{k}}+3\right)} \cdot \gamma_{0}^{2} \cdot \mathcal{I}_{0}
\end{aligned}
$$


und für Anti-Stokes-Übergänge: ${ }^{26}$

$$
\begin{aligned}
& I^{\mathrm{AS}}\left(\pi / 2, \perp^{s}+\|^{s}, \perp^{i}\right)= \\
& \quad \frac{\pi^{2}}{6 \epsilon_{0}^{2}} \cdot g_{\mathrm{N}} \cdot N\left(\widetilde{\nu}_{0}+\widetilde{\nu}_{\mathrm{k}}\right)^{4} \frac{\exp \left\{-B \cdot J_{\mathrm{k}}\left(J_{\mathrm{k}}+1\right) h c_{0} / k T\right\}}{Z_{\mathrm{rot}}} \frac{J_{\mathrm{k}}\left(J_{\mathrm{k}}-1\right)}{\left(2 J_{\mathrm{k}}-1\right)} \cdot \gamma_{0}^{2} \cdot \mathcal{I}_{0}
\end{aligned}
$$

Mit $\widetilde{\nu}_{\mathrm{k}}$ als Wellenzahl des k-ten Rotationsübergangs und $\gamma_{0}$ der symmetrischen Anisotropie im Schwingungsgrundzustand.

\subsubsection{Depolarisation}

Das Depolarisationsverhältnis $\delta$ ist das Intensitätsverhältnis der senkrecht und parallel zur Streuebene polarisierten Streustrahlung. Es ist charakteristisch für einen Schwingungsübergang und folgendermaßen mit den abgeleiteten Invarianten des Polarisierbarkeitstensors ${ }^{8-10,26}$ verknüpft:

$$
\delta=\frac{I\left(\pi / 2, \|^{s}, \perp^{i}\right)}{I\left(\pi / 2, \perp^{s}, \perp^{i}\right)}=\frac{3 \gamma_{\mathrm{k}}^{\prime 2}}{45 a_{\mathrm{k}}^{\prime 2}+4 \gamma_{\mathrm{k}}^{\prime 2}} \leq 0.75
$$

Experimentell kann das Depolarisationsverhältnis durch Einsatz eines Polarisationsfilters ermittelt werden, der jeweils nur eine Polarisationsrichtung und daher nur einen Teil des Streulichts durchlässt. Der Nachteil dieser Methode ist, dass hierdurch ein Teil des Signals verloren geht. Daher empfiehlt es sich, stattdessen die Polarisationsrichtung des einfallenden Lichts mithilfe eines $\lambda / 2$-Plättchens zu drehen, so dass es einmal senkrecht und einmal parallel zur Streuebene polarisiert ist. ${ }^{8}$ Detektiert wird sowohl das senkrecht und als auch das parallel zur Streuebene polarisierte Streulicht. Hierdurch ergibt sich das Depolarisationsverhältnis $\delta^{\prime}: 9,10,26$

$$
\delta^{\prime}=\frac{I\left(\pi / 2, \perp^{s}+\left\|^{s},\right\|^{i}\right)}{I\left(\pi / 2, \perp^{s}+\|^{s}, \perp^{i}\right)}=\frac{6 \gamma_{\mathrm{k}}^{\prime 2}}{45 a_{\mathrm{k}}^{\prime 2}+7 \gamma_{\mathrm{k}}^{\prime 2}} \leq 0.857
$$

Die senkrecht und parallel zur Streuebene polarisierten Anteile der Streustrahlung ergeben sich wie folgt: ${ }^{26}$

$$
\begin{aligned}
I\left(\pi / 2, \perp^{s}, \|^{i}\right) & =3 \gamma_{\mathrm{k}}^{\prime 2} \\
I\left(\pi / 2,\left\|^{s},\right\|^{i}\right) & =3 \gamma_{\mathrm{k}}^{\prime 2} \\
I\left(\pi / 2, \perp^{s}, \perp^{i}\right) & =45 a_{\mathrm{k}}^{\prime 2}+4 \gamma_{\mathrm{k}}^{\prime 2} \\
I\left(\pi / 2, \|^{s}, \perp^{i}\right) & =3 \gamma_{\mathrm{k}}^{\prime 2}
\end{aligned}
$$

Durch Vergleich der experimentell bestimmten Depolarisationsverhältnisse mit den aus quantenchemischen Rechnungen erhaltenen kann die Zuordnung der Schwingungsban- 
den erleichtert werden. Insbesondere totalsymmetrische Schwingungen, bei denen die Polarisationsrichtung des eingestrahlten Lichts beim Streuvorgang weitgehend erhalten bleibt, können so eindeutig ermittelt werden.

Um berechnete und experimentell ermittelte Depolarisationsverhältnisse vergleichen zu können, muss allerdings die Effizienz des Monochromatorgitters für senkrecht und parallel zur Streuebene polarisiertes Licht einbezogen werden. ${ }^{11}$ Der verwendete Monochromator (McPherson Model 2051 s. auch Kap. 3) ist für senkrecht zur Streuebene polarisiertes Licht je nach Wellenlänge um 1.5-2.5 mal effizienter. In Ref. 11 findet sich eine Eichkurve, die Gewichtungsfaktoren für die beiden Polarisationsrichtungen ausgibt. Mithilfe dieser Kurve können die berechneten Streuquerschnitte und Depolarisationsverhältnisse an das in diesem Fall verwendete Experiment angepasst werden.

\subsection{Temperaturbestimmung}

Die effektive Temperatur sowohl von einer Schwingung als auch von einer Rotation kann aus dem Verhältnis der Intensitäten der Stokes- und Anti-Stokes-Übergänge bestimmt werden. Es gilt für eine Energiedichtemessung: ${ }^{26}$

$$
\frac{I^{\mathrm{S}}}{I^{\mathrm{AS}}}=\frac{\left(\widetilde{\nu}_{0}-\widetilde{\nu}_{\mathrm{k}}\right)^{4}}{\left(\widetilde{\nu}_{0}+\widetilde{\nu}_{\mathrm{k}}\right)^{4}} \cdot \exp \left\{\frac{h c_{0} \widetilde{\nu}_{\mathrm{k}}}{k T}\right\}
$$

bzw. für die Messmethode des Photonenzählens, ${ }^{9}$ die in dieser Arbeit angewendet wurde:

$$
\frac{I^{\mathrm{S}}}{I^{\mathrm{AS}}}=\frac{\left(\widetilde{\nu}_{0}-\widetilde{\nu}_{\mathrm{k}}\right)^{3}}{\left(\widetilde{\nu}_{0}+\widetilde{\nu}_{\mathrm{k}}\right)^{3}} \cdot \exp \left\{\frac{h c_{0} \widetilde{\nu}_{\mathrm{k}}}{k T}\right\}
$$

Daraus folgt für die Temperatur

$$
T=\frac{h c_{0} \widetilde{\nu}_{\mathrm{k}}}{\left(\ln \frac{I^{\mathrm{S}}}{I^{\mathrm{AS}}}-\ln \frac{\left(\widetilde{\nu}_{0}-\widetilde{\nu}_{\mathrm{k}}\right)^{3}}{\left(\widetilde{\nu}_{0}+\widetilde{\nu}_{\mathrm{k}}\right)^{3}}\right) \cdot k}
$$

Für die Rotationstemperatur können für den Fall eines zweiatomigen homonuklearen Moleküls (wie z.B. $\mathrm{N}_{2}$ ) die in Kapitel 2.2.2 gezeigten Formeln verwendet werden. Zur Vereinfachung werden alle Werte, die sich für verschiedene Rotationsübergänge nicht ändern, in einer Konstante $C$ zusammengefasst. Es ergeben sich folgende vereinfachte Formeln (ebenfalls für das Photonenzählen als Messmethode)

Für Stokes-Rotationslinien:

$$
I^{\mathrm{S}}=C \cdot g_{N}\left(\widetilde{\nu}_{0}-\widetilde{\nu}_{\mathrm{k}}\right)^{3} \frac{\left(J_{\mathrm{k}}+1\right)\left(J_{\mathrm{k}}+2\right)}{\left(2 J_{\mathrm{k}}+3\right)} \cdot \exp \left(-B \cdot J_{\mathrm{k}}\left(J_{\mathrm{k}}+1\right) h c_{0} / k T\right)
$$


und für Anti-Stokes-Rotationslinien:

$$
I^{\mathrm{AS}}=C \cdot g_{N}\left(\widetilde{\nu}_{0}+\widetilde{\nu}_{\mathrm{k}}\right)^{3} \frac{J_{\mathrm{k}}\left(J_{\mathrm{k}}-1\right)}{\left(2 J_{\mathrm{k}}-1\right)} \cdot \exp \left(-B \cdot J_{\mathrm{k}}\left(J_{\mathrm{k}}+1\right) h c_{0} / k T\right)
$$

Der Einfluss des Faktors $\left(\widetilde{\nu}_{0} \pm \widetilde{\nu}_{\mathrm{k}}\right)^{3}$ ist für Rotationsübergänge $\left(\widetilde{\nu}_{\mathrm{k}} \ll \widetilde{\nu}_{0}\right)$ relativ gering und wird somit mit der Konstante $C$ zusammengefasst. ${ }^{a}$

Daher kann aus einer Auftragung von $\ln \left(\frac{2 J_{\mathrm{k}}+3}{\left(J_{\mathrm{k}}+1\right)\left(J_{\mathrm{k}}+2\right)} \cdot I^{\mathrm{S}}\right)$ bzw. von $\ln \left(\frac{2 J_{\mathrm{k}}-1}{J_{\mathrm{k}} \cdot\left(J_{\mathrm{k}}-1\right)} \cdot I^{\mathrm{AS}}\right)$ gegen $J_{\mathrm{k}}\left(J_{\mathrm{k}}+1\right)$ aus der Steigung $m$ die Temperatur bestimmt werden.

$$
\begin{aligned}
& m=-\frac{h c_{0} B}{k T} \\
& T=-\frac{h c_{0} B}{k \cdot m}
\end{aligned}
$$

Für homonukleare zweiatomige Moleküle muss außerdem die Kernspinstatistik beachtet werden, die die unterschiedliche Besetzung von Zuständen mit gerader und ungerader Kernspinwellenfunktion beschreibt. Bei optischen Übergängen kann sich die Parität der Kernspinwellenfunktion nicht ändern, daher müssen Zustände mit geraden und ungeraden Kernspinwellenfunktionen getrennt voneinander betrachtet werden. ${ }^{9,36}$

\subsection{Davydov-Aufspaltung in symmetrischen Clustern}

Der Begriff Davydov-Aufspaltung rührt von der Aufspaltung von elektronischen oder Schwingungsbanden in symmetrischen Molekülkristallen her. Diese entstehen aufgrund von Wechselwirkungen zwischen mehreren äquivalenten molekularen Einheiten. ${ }^{8,10}$ In symmetrischen Molekülaggregaten werden in Analogie äquivalente Oszillatoren betrachtet, deren Kopplung ebenfalls zu einer Aufspaltung der Schwingungsniveaus führt (Schwingungsexcitonenmodell). Die Kopplung wird nachfolgend auch als Excitonenkopplung bezeichnet. Im Folgenden werden die Aufspaltungen und die hieraus resultierenden Kopplungskonstanten für cyclische Trimere, Tetramere und Pentamere kurz dargestellt. Zur Beschreibung der Kopplungen wird ein Ansatz verwendet, der ähnlich dem Hückel-Modell zur Beschreibung von Molekülorbitalen in konjugierten $\pi$-Systemen ist. Ausführlichere Herleitungen finden sich in Ref. 8,10,12,15.

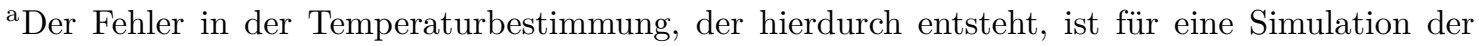
Stickstoffrotationslinien im thermischen Gleichgewicht bei $300 \mathrm{~K} \mathrm{zu} \leq 2 \mathrm{~K}$ bestimmt worden. Somit ist der Fehler, der durch diese Vereinfachung entsteht, deutlich geringer als die durch das Experiment hervorgerufenen Fehler (wie z.B. Fehler durch unterschiedliche Pixelsensitivitäten.) 


\subsubsection{Trimer}

Für cyclische Trimere müssen zwei verschiedene Kopplungsfälle betrachtet werden zum einen der symmetrische, bei dem alle Kopplungen äquivalent sind, und zum anderen der asymmetrische Fall, bei dem sich die Stärke der Kopplungen unterscheidet. Im symmetrischen Fall ergibt sich eine $C_{3}$-Symmetrie und somit folgende Determinantengleichung:

$$
\left|\begin{array}{ccc}
x & W & W \\
W & x & W \\
W & W & x
\end{array}\right|=0
$$

Deren Lösungen sind:

$$
\begin{array}{rlll}
x_{1} & = & -2 W & (\mathrm{~A}) \\
x_{2,3} & =W & (\mathrm{E})
\end{array}
$$

Somit ergibt sich also eine A-symmetrische stark Raman-aktive Schwingung und eine zweifach entartete stark IR-aktive Schwingung (E-Symmetrie). Die Aufspaltung zwischen den Niveaus beträgt $3 W$. Die Kopplungskonstante $W$ kann also aus dem Abstand der beiden Banden dividiert durch drei ermittelt werden.

Für den asymmetrischen Fall werden zwei verschiedene Kopplungskonstanten $W_{1}$ und $W_{2}$ angenommen. Die Determinantengleichung verändert sich zu:

$$
\left|\begin{array}{ccc}
x & W_{1} & W_{2} \\
W_{1} & x & W_{1} \\
W_{2} & W_{1} & x
\end{array}\right|=0
$$

Als Lösungen ergeben sich:

$$
\begin{aligned}
& x_{1}=-\frac{\sqrt{8 W_{1}^{2}+W_{2}^{2}}}{2}-\frac{W_{2}}{2} \\
& x_{2}=\quad W_{2} \\
& x_{3}=\frac{\sqrt{8 W_{1}^{2}+W_{2}^{2}}}{2}-\frac{W_{2}}{2}
\end{aligned}
$$

Die Aufspaltung zwischen dem höchsten und dem niedrigsten Niveau beträgt $\sqrt{8 W_{1}^{2}+W_{2}^{2}}$, das mittlere Niveau ist vom Schwerpunkt des Multipletts um $W_{2}$ verschoben. 


\subsubsection{Tetramer}

Für cyclische Tetramere sollen hier $S_{4}$-symmetrische Aggregate betrachtet werden. Neben der Kopplung zum benachbarten Oszillator muss zusätzlich die Kopplung zum zweitnächsten Oszillator berücksichtigt werden. Daher ergeben sich auch hier zwei verschiedene Kopplungskonstanten $W_{1}$ und $W_{2}$. Die Determinante der Kopplungsmatrix

$$
\left|\begin{array}{cccc}
x & W_{1} & W_{2} & W_{1} \\
W_{1} & x & W_{1} & W_{2} \\
W_{2} & W_{1} & x & W_{1} \\
W_{1} & W_{2} & W_{1} & x
\end{array}\right|=0
$$

besitzt die Lösungen:

$$
\begin{array}{cccc}
x_{1} & = & -2 W_{1}-W_{2} & (\mathrm{~A}) \\
x_{2,3} & = & W_{2} & (\mathrm{E}) \\
x_{4} & = & 2 W_{1}-W_{2} & (\mathrm{~B})
\end{array}
$$

Es ergibt sich somit eine stark Raman-aktive A-symmetrische Schwingung, eine zweifach entartete, stark IR-aktive Schwingung und eine schwach Raman-aktive B-symmetrische Schwingung.

\subsubsection{Pentamer}

Für cyclische Pentamere soll hier nur der Kopplungsfall für $C_{5 h}$-symmetrische Aggregate betrachtet werden. Auch hier muss zwischen der Kopplung zum Nachbaroszillator und zum zweiten Nachbaroszillator unterschieden werden. Als Determinante der Kopplungsmatrix ergibt sich:

$$
\left|\begin{array}{ccccc}
x & W_{1} & W_{2} & W_{2} & W_{1} \\
W_{1} & x & W_{1} & W_{2} & W_{2} \\
W_{2} & W_{1} & x & W_{1} & W_{2} \\
W_{2} & W_{2} & W_{1} & x & W_{1} \\
W_{1} & W_{2} & W_{2} & W_{1} & x
\end{array}\right|=0
$$

Als Lösungen der Determinantengleichung $x_{i}$ relativ zum Schwerpunkt des Multipletts (mit $g=\frac{1}{g}-1=(\sqrt{5}-1) / 2$, dem goldenen Schnitt) ergeben sich:

$$
\begin{array}{rll}
x_{1} & =-2 W_{1}-2 W_{2} & \left(\mathrm{~A}^{\prime}\right) \\
x_{2,3} & =-g W_{1}+W_{2} / g & \left(\mathrm{E}_{1}^{\prime}\right) \\
x_{4,5} & =W_{1} / g-g W_{2} & \left(\mathrm{E}_{2}^{\prime}\right)
\end{array}
$$


Die Aufspaltung zwischen der intensiven IR-Bande $\left(\mathrm{E}_{1}^{\prime}\right)$ und der intensiven RamanBande $\left(\mathrm{A}^{\prime}\right)$ beträgt $(2-g) W_{1}+(3+g) W_{2}$, wohingegen die Aufspaltung zwischen den beiden Raman-aktiven Banden $(3+g) W_{1}+(2-g) W_{2}$ ist. Bei Auslenkung aus der $C_{5 h^{-}}$ Symmetrie, wie beispielsweise im Wasserpentamer, ${ }^{38}$ werden die entarteten Niveaus noch weiter aufgespalten, allerdings nur um einen kleinen Betrag.

\subsection{Optisch parametrischer Oszillator}

In der vorliegenden Arbeit wurde ein IR-Laser verwendet, der aufgrund von optisch parametrischer Oszillation durchstimmbar ist. Im Folgenden soll kurz erläutert werden, wie die Strahlung in diesem Laser erzeugt wird. Im Wesentlichen wird die intensive Strahlung eines Pumplasers in zwei Teilstrahlen aufgeteilt. Diese Aufteilung erfolgt durch den parametrischen Prozess, der eine nichtlineare Wechselwirkung dreier elektromagnetischer Wellen darstellt. ${ }^{39}$

Wird ein Pumpstrahl hoher Energie in ein optisch nichtlineares Medium eingestrahlt, so ergibt sich eine inelastische Streuung der Pumpphotonen mit der Kreisfrequenz $\omega_{\mathrm{p}}$, die dazu führt, dass zwei neue Strahlen, der Idler- $\left(\omega_{\mathrm{i}}\right)$ und der Signalstrahl $\left(\omega_{\mathrm{s}}\right)$, generiert werden. ${ }^{39,40}$ Hierbei gilt sowohl Energie- als auch Impulserhaltung mit

$$
\omega_{\mathrm{p}}=\omega_{\mathrm{s}}+\omega_{\mathrm{i}}
$$

und

$$
k_{\mathrm{p}}=k_{\mathrm{s}}+k_{\mathrm{i}}
$$

Wobei $k_{\mathrm{p}, \mathrm{s}, \mathrm{i}}$ der Wellenvektor des Pump- (p), Signal- (s) und Idlerphotons (i) ist. Die Auswahl eines bestimmten Signal-Idler-Paares, für das eine Verstärkung auftritt, kann durch die Wahl des Winkels, in dem die Pumpwelle in Bezug auf die Kristallachse eingestrahlt wird, erfolgen. ${ }^{39,41}$ Die größte Effizienz erhält man aber bei kollinearer Phasenanpassung. Diese kann realisiert werden, wenn die Pumpwelle als ordentlicher Strahl einfällt, während die erzeugten Wellen außerordentliche Strahlen sind. Für größtmögliche Effizienz müssen Pump- und Signal/Idlerwelle senkrecht zueinander polarisiert sein. ${ }^{40,42}$ Ein Nachteil dieser Methode ist, dass es nur einen geringen Winkelbereich gibt, in dem der Laser durchgestimmt werden kann, ohne dass größere Verluste auftreten. ${ }^{43}$ Weiterhin ist der erreichbare Wellenlängenbereich stark von den zugänglichen Kristallmaterialien abhängig.

Eine weit robustere Methode zur Verstärkung der entstandenen Wellenlängen ist die Quasiphasenanpassung (QPM). Hierbei werden die Kristalleigenschaften periodisch so modifiziert, dass für eine bestimmte Wellenlänge immer eine konstruktive Interferenz gewährleistet ist. ${ }^{43}$ Die Kristalle weisen somit eine Domänenstruktur auf. Die Inversion 


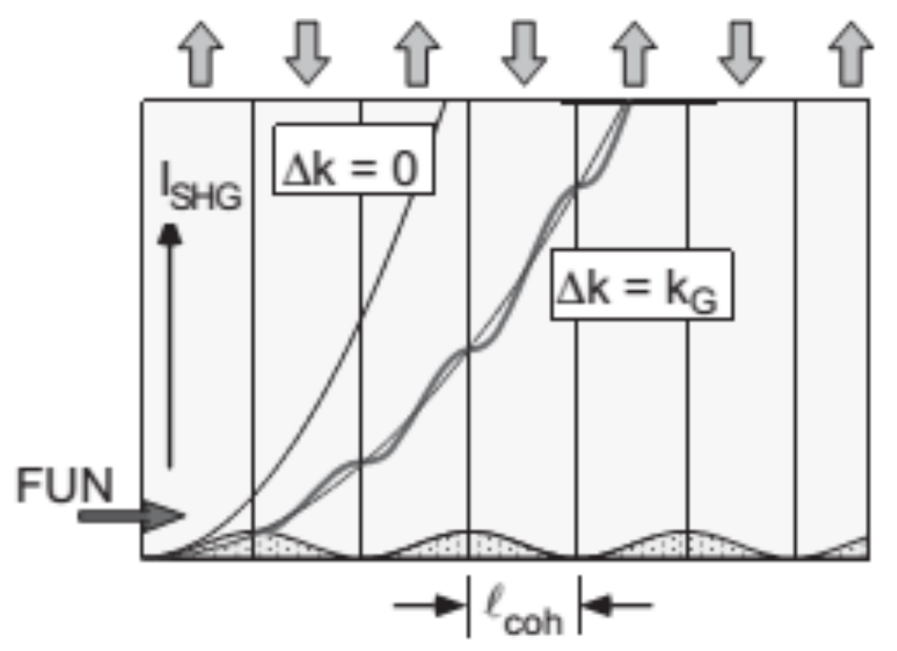

Abbildung 2.1: Intensitätsverlauf für perfekte Phasenanpassung $(\Delta k=0)$, Quasiphasenanpassung $\left(\Delta k=k_{\mathrm{G}}\right)$ und ohne Phasenanpassung (unten). ${ }^{44}$ Die Bezeichnung „FUN“ gibt die Einstrahlungsrichtung des Pumplasers an. Die Domänenstruktur des Kristalls ist im oberen Bereich der Abbildung mit Pfeilen dargestellt. Die Dicke der Domänen entspricht einer Kohärenzlänge $l_{\text {coh}}$. Abbildung entnommen aus Ref. 44.

der Kristalleigenschaften erfolgt nach einer Kohärenzlänge $l_{\text {coh }}$. Diese legt daher die Dicke der Domänen fest. ${ }^{44}$

Abbildung 2.1 zeigt den räumlichen Intensitätsverlauf der erzeugten Strahlung bei perfekter Phasenanpassung $(\Delta k=0)$, Quasiphasenanpassung $\left(\Delta k=k_{\mathrm{G}}\right)$ und für nicht phasenangepasste Photonen (unten). ${ }^{44}$ Die Domänenstruktur ist mit Pfeilen angedeutet. Für perfekte Phasenanpassung erfolgt der Intensitätsanstieg quadratisch mit der Kristalllänge, während sich für die Quasiphasenanpassung ein geringerer Anstieg der Intensität ergibt. ${ }^{44}$ Die Vorteile der Quasiphasenanpassung liegen allerdings darin, dass auch für große Kristalllängen eine kollineare Quasiphasenanpassung erreicht werden kann. ${ }^{39}$ Außerdem müssen die drei Strahlen (Pump, Signal und Idler) für eine große Konversionseffizienz keine festgelegten Polarisationsrichtungen zueinander haben. ${ }^{45} \mathrm{Zu}-$ dem können durch eine geeignete Wahl der Domänendicke Wellenlängen im gesamten Bereich, in dem der Kristall durchlässig ist, erzeugt werden. ${ }^{46}$

Der für diese Arbeit verwendete Laser besitzt einen periodisch gepolten $\mathrm{LiNbO}_{3}$-Kristall (PPLN), der über ferroelektrische Domänen mit alternierender Polarität verfügt. ${ }^{40,43}$ Diese Kristalle können so gefertigt werden, dass sie verschiedene Bereiche mit unterschiedlichen Perioden aufweisen. Daher können durch das laterale Verschieben des Kristalls im Bezug auf den Pumplaser verschiedene Signal-Idler-Paare generiert werden. Zusätzlich sind durch Temperaturänderung des Kristalls noch weitere Signal-Idler-Paare zugänglich. ${ }^{43}$ 


\section{Experimentelles}

\subsection{Curry-Jet Apparatur - Allgemeines}

Zur Aufnahme der in dieser Arbeit verwendeten Spektren wurde die Curry-Jet Apparatur verwendet, mit der Schwingungen und Rotationen kalter, isolierter Moleküle und Molekülaggregate untersucht werden können. Die Apparatur wurde von P. Zielke entwickelt $^{8}$ und von T. Wassermann ${ }^{9}$ und Z. Xue ${ }^{10}$ zunächst bezüglich des Signalzu-Rausch-Verhältnisses verbessert. Durch N. Lüttschwager wurde die Apparatur so umgebaut, dass auch schwerflüchtige Substanzen untersucht werden können. ${ }^{11}$ Die genannten Referenzen beinhalten ausführliche Beschreibungen der Apparatur, daher wird im Folgenden nur eine kurze Zusammenfassung der verschiedenen Messmodi gegeben. In Abbildung 3.1 ist die Apparatur schematisch gezeigt. Die zu messenden Substanzen werden in einen temperierten mit Trägergas durchströmten Glassättiger gefüllt, wodurch ein Substanz-Trägergas-Gemisch entsteht. Das Gemisch wird zunächst in einem Reservoir (4.8 L, Teflon-beschichtet) gelagert und anschließend durch eine Schlitzdüse in eine Vakuumkammer expandiert. Es stehen Düsen mit verschiedene Abmessungen zur Verfügung. Wenn nicht eigens im Spektrenverzeichnis (Anhang A.3) angegeben, wurde in der vorliegenden Arbeit eine $4 \times 0.15 \mathrm{~mm}^{2}$ Düse verwendet.

Die Raman-Anregung erfolgt mittels eines grünen Dauerstrichlasers (Coherent Verdi V18, $532 \mathrm{~nm}$ ) mit $18 \mathrm{~W}$ Maximalleistung. Dieser wird mithilfe einer Plankonvexlinse (Qioptiq, Nr 312-300-322, $\varnothing=22.4 \mathrm{~mm}, f=50 \mathrm{~mm}$, Beschichtung ARB2) senkrecht zur Expansionsrichtung auf die Expansion fokussiert. Das gestreute Licht wird im $90^{\circ}-$ Winkel zur Expansion und zur Anregung mit einem Objektiv (Nikon Nikkor $50 \mathrm{~mm}$, $f / 1.2$ ) kollimiert und danach mit einer achromatischen Sammellinse (für Monochromator Model 2051: Edmund Optics GmbH Typ L45-354, $\varnothing=50 \mathrm{~mm}, f=350 \mathrm{~mm}$, $f / 7$; für Model 205f: Newport GmbH, Typ PAC087AR.14, $\varnothing=50.8 \mathrm{~mm}, f=200 \mathrm{~mm}$, $f / 3.9$ ) auf den Eintrittspalt (Spaltbreite $75 \mu \mathrm{m}$ ) eines Monochromators fokussiert. Die Rayleigh-Streuung wird mithilfe eines Kantenfilters entfernt, je nach Messmethode stehen ein Stokes- und ein Anti-Stokes-Kantenfilter zur Verfügung (Angaben über die Spezifikationen der Filter finden sich in Kap. A.4 im Anhang). Die Detektion des Lichtes erfolgt mit einer durch Flüssigstickstoff gekühlten CCD-Kamera (Princeton Instruments Digital Spectroscopy, Spec-10, 400B/LN) mit $1340 \times 400$ Pixeln. Die Belichtungszeiten der Kamera belaufen sich meist auf 1-10 min pro Aufnahme. 


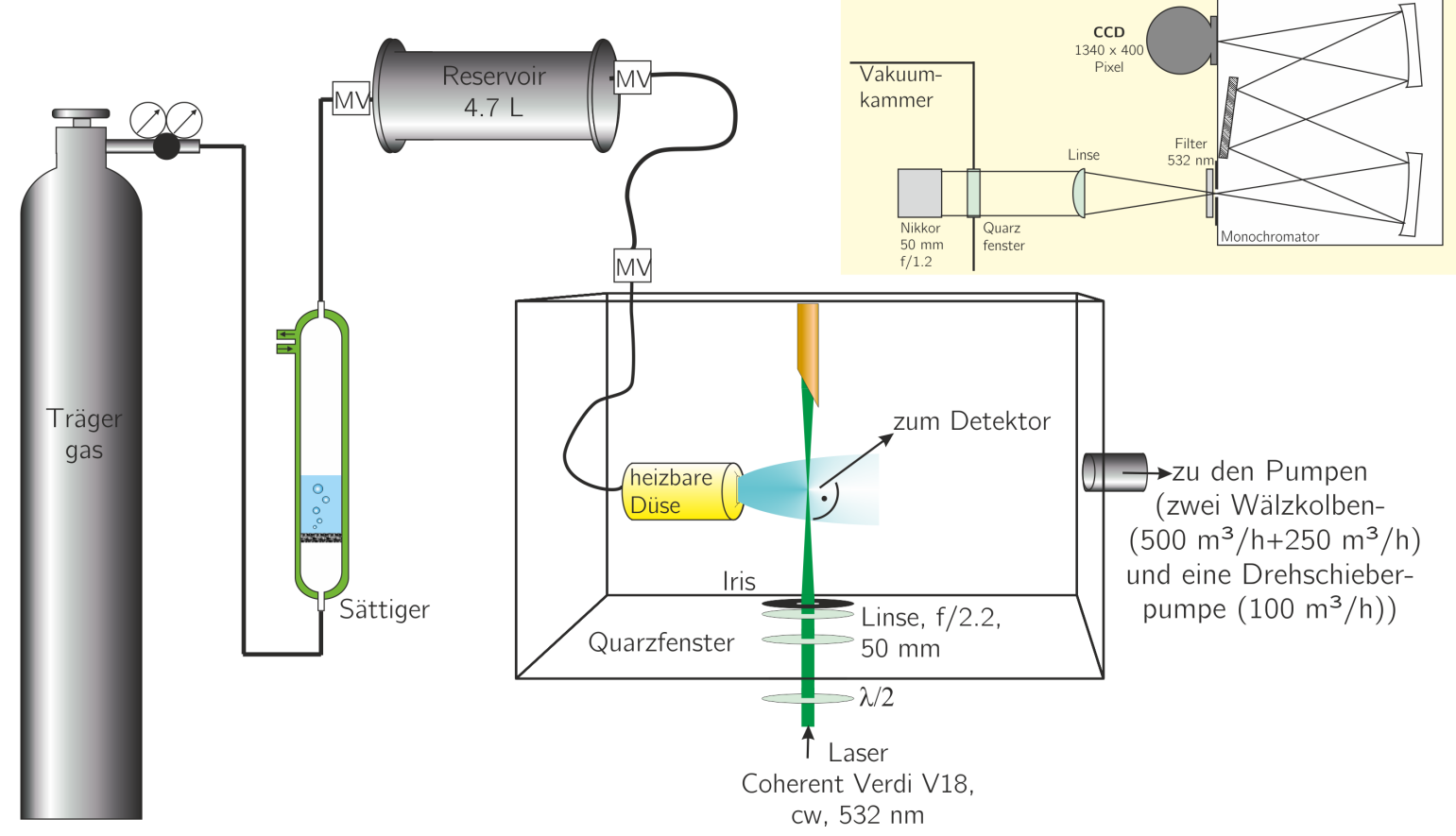

Abbildung 3.1: Schematische Darstellung der Curry-Jet Apparatur.

Es stehen zwei verschiedene Monochromatoren zur Verfügung und zwar ein Monochromator mit einer Brennweite von $1 \mathrm{~m}$ (McPherson Inc. Model 2051, f/8.7, Gitter 1200 Linien/mm) und einer mit $0.5 \mathrm{~m}$ Brennweite (McPherson Inc. Model 205f, f/3.2), der zwei verschiedene Gitter besitzt (600 und 1800 Linien/mm). In der vorliegenden Arbeit wurde der McPherson 205f Monochromator ausschließlich mit dem gröberen Gitter mit 600 Linien/mm verwendet, mit dem sich eine Auflösung von $6-8 \mathrm{~cm}^{-1}$ ergibt. Der McPherson Model 2051 Monochromator hat eine Auflösung von 1-2 $\mathrm{cm}^{-1}$. (Zur Abschätzung der Auflösung wurden die vollen Halbwertsbreiten der intensiven Ne-Linien im Bereich eines Stokes-Raman-Shifts von $3600 \mathrm{~cm}^{-1}$ und nahe der Rayleighlinie um $288 \mathrm{~cm}^{-1}$ jeweils bei einer Monochromator-Spaltbreite von $75 \mu \mathrm{m}$ bestimmt.)

Standardmäßig wird der Monochromator mit besserer Auflösung verwendet. Der Vorteil des kürzeren Monochromators ist jedoch, dass mit ihm Übersichtsspektren, die einen Bereich von $2000 \mathrm{~cm}^{-1}$ umfassen, aufgenommen werden können, wodurch erschlossen werden kann in welchen Bereichen es sinnvoll ist ergänzende Spektren mit höherer Auflösung aufzunehmen.

Zur Signaloptimierung wird eine Expansion von Raumluft genutzt und das Signal des Q-Zweigs der Stickstoffschwingung maximiert. Hierzu können das Objektiv in xyzRichtung und die Düse in yz-Richtung mithilfe von Präzisionselektromotoren (min. Schrittweite $0.5 \mu \mathrm{m}$ ) verschoben werden (siehe auch Ref. 9). Der Abstand zwischen Raman-Anregungslaser und Düse kann über einen Präzisionsschrittmotor (min. Schritt- 
weite $0.1 \mu \mathrm{m}$ ) eingestellt werden. Hierzu muss zunächst die Nullposition der Düse bekannt sein, die wie in Ref. 9 beschrieben bestimmt wurde.

Die Kalibrierung der Spektren erfolgte mit einem Neonlinienstrahler (L.O.T.-Oriel GmbH \& Co. KG, Typ LSP032). Außerdem müssen aus den Rohdaten noch sog. Spikes, hochenergetische meist ionische Strahlung, die statistisch während der Messung auf den Detektor trifft, entfernt werden. Mittels eines von N. Lüttschwager geschriebenen Matlab-Skripts, ${ }^{11}$ das nach einem von P. Zielke entwickelten Algorithmus ${ }^{8}$ arbeitet, werden hintereinander aufgenommene Einzelspektren verglichen und gemittelt, bei Abweichungen der Intensität eines Datenpunkts in einem Spektrum oberhalb eines vom Anwender definierten Schwellenwerts wird die Intensität auf die mittlere Intensität aller anderen Spektren gesetzt. Durch eine iterative Anwendung des Verfahren mit sukzessiver Erniedrigung des Schwellenwerts können i.d.R. alle Spikes entfernt werden. Das Verfahren erfordert für eine effiziente Anwendung die Aufnahme von mindestens 4 Einzelspektren hintereinander. ${ }^{11}$

Abweichend von der oben beschriebenen Methode kann die Apparatur noch in anderen Messmodi verwendet werden. Beispielsweise kann allein die Düse geheizt werden, oder aber es können durch Verwendung eines heizbaren Sättigers schwerflüchtige Substanzen untersucht werden. Eine ausführliche Beschreibung der heizbaren Düse und des Sättigers findet sich in Ref. 11.

Durch das Heizen der Düse kann die Clusterbildung je nach Temperatur teilweise oder vollständig unterdrückt werden. Durch Aufnahme mehrerer Spektren bei verschiedenen Düsentemperaturen kann die Zuordnung der Peaks zu verschiedenen Clustergrößen erleichtert werden. In der vorliegenden Arbeit wurde die heizbare Düse außerdem dazu genutzt, das Konformerengleichgewicht von Monomerkonformeren vor der Expansion $\mathrm{zu}$ verschieben und die Auswirkungen auf das Jetspektrum zu evaluieren.

Für Messungen schwerflüchtiger Substanzen wird ein heizbarer Metallsättiger zwischen Reservoir und Jetkammer geschaltet. In ihm wird die Substanz vorgelegt und auf bis zu $160^{\circ} \mathrm{C}$ erhitzt. Außerdem werden alle Schlauchverbindungen vom Reservoir bis zur Düse geheizt. Hierbei ist darauf zu achten, dass am Sättiger die niedrigste Temperatur eingestellt ist, so dass, falls es doch zu einer Kondensation kommt, diese vorrangig im Sättiger geschieht. Es können hierbei einige Probleme auftreten, die in Ref. 11 ausführlich diskutiert sind. Der Vollständigkeit halber werden sie hier noch einmal erwähnt. Beim Erhitzen der Düse dehnt diese sich aus, daher muss die Düsennullposition für verschiedene Düsentemperaturen immer wieder neu bestimmt werden. Des Weiteren kann durch den Wärmeaufschlag in der Kammer ein passives Heizen besonders des Objektivs erfolgen, wodurch eine erneute Justierung notwendig sein kann. (Bei ungeheizter Düse ist eine Justierung zu Beginn des Messtages vollkommen ausreichend.) Bei Verwendung hoher Substanzkonzentrationen können sich die schwerflüchtigen Substanzen in den Schlauchleitungen und auf den Optikkomponenten absetzen, was teils recht aufwändige Reinigungsmaßnahmen notwendig macht. 


\subsection{Eigenschaften der neuen Edelstahlexpansionskammer}

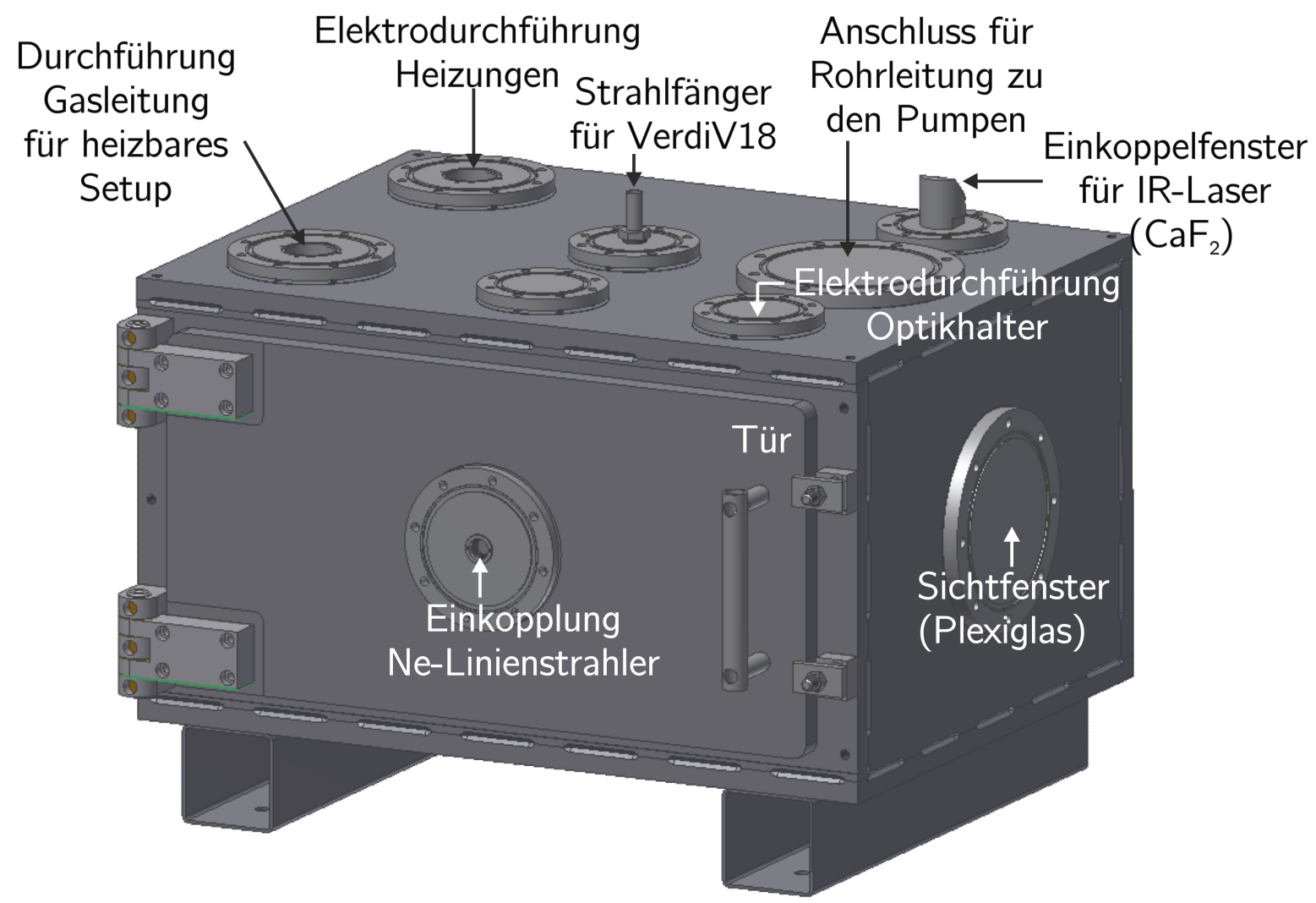

Abbildung 3.2: Technische 3D-Darstellung ${ }^{47}$ der Edelstahlvakuumkammer. Neben den schon vorhandenen Durchführungen und Fenstern für die in Kapitel 3.1 beschriebenen Nutzungsmöglichkeiten der Apparatur, ist nun auch die Einkopplung eines IR-Lasers über ein entsprechendes Einkoppelfenster möglich.

Die in Kapitel 3.1 beschriebene Apparatur ist mit einer Vakuumkammer aus schwarz eloxiertem Aluminium ausgestattet. Diese musste im Laufe dieser Arbeit ausgetauscht werden, da die Anzahl an Flanschen nicht ausreichte um die Einkopplung eines IRLasers zu realisieren. Außerdem wies die Aluminiumkammer undichte Schweißnähte auf, so dass Messungen von Gasphasen unter Gleichgewichtsbedingungen nur bei kurzen Messzeiten möglich waren. Eine technische Zeichnung ${ }^{47}$ der neuen Vakuumkammer ist in Abbildung 3.2 gezeigt. Sie besteht aus Edelstahl und besitzt zur Streulichtunterdrückung innen eine schwarze Lackierung (Kunstschmiede-Mattlack, PU-Alkydharzlack, Farbe: schwarz, P. A. Jansen GmbH \& Co., KG). ${ }^{48}$ Die Abmessungen der Kammer betragen $80 \times 40 \times 60 \mathrm{~cm}^{3}(B \times H \times T)$. Die Anzahl der Flansche wurde auf 14 erhöht und damit im Vergleich zur Aluminiumkammer fast verdoppelt, außerdem verfügt die 
Edelstahlkammer über eine Tür, die ein sehr komfortables Arbeiten bei Umbauten und Justierung in der Kammer ermöglicht. Die Geometrie von Anregung, Expansion und Detektion wurde beibehalten. Im Unterschied zu der Aluminiumkammer befindet sich der Anschluss für die Pumpen an der Oberseite der Kammer. Außerdem kann einer der Flansche (s. Abb. 3.2) durch ein Sichtfenster aus Plexiglas ${ }^{\circledR}$ ausgetauscht werden. Reflexionen am Fenster führen jedoch zu einer leichten Erniedrigung des Signal-zuRausch-Verhältnisses (um ca. $5 \%$ ). Bei ersten Testmessungen, bei denen zunächst nur starke Raman-Streuer untersucht wurden, konnten hierdurch aber keine Einschränkungen ermittelt werden. Hingegen sollte bei späteren Routinemessungen auf das Sichtfenster verzichtet werden. Wie in Abbildung 3.2 eingezeichnet kann an der Oberseite der Kammer ein Einkoppelfenster aus $\mathrm{CaF}_{2}$ angebracht werden. Eine Elektrodurchführung ermöglicht die Steuerung der Optiken, die für die Nutzung des IR-Lasers erforderlich sind.

Zur Ermittlung der Dichtigkeit wurde die Kammer vollständig evakuiert und der Druckanstieg über 24 Stunden beobachtet. Es wurde eine Leckrate von $<0.015 \mathrm{mbar} \mathrm{h}^{-1}$ bestimmt. Im Vergleich zur Aluminiumkammer ${ }^{9}$ ist diese um fast 2 Größenordnungen kleiner. Im Zuge des Umbaus wurde auch der Pumpstand aus dem Messraum in einen Lüftungsschacht verlegt, so dass die Wärmebelastung im Messlabor deutlich verringert werden konnte. Der durchschnittliche Hintergrunddruck $\left(p_{\mathrm{b}}\right)$ bei Expansion der Umgebungsluft (Stagnationsdruck $p_{\mathrm{S}} \approx 1$ bar) ist mit 0.86 mbar um ca. $15 \%$ höher als bei der alten Kammer. Diese Erhöhung ist wahrscheinlich vorwiegend auf den längeren Rohrleitungsweg zurückzuführen. Als Maß für die Saugleistung in einer Vakuumapparatur gilt der Leitwert $L$, dieser hängt maßgeblich von Länge und Durchmesser sowie der Anzahl an Umlenkungen in der Rohrleitung ab. Der Gesamtleitwert $L_{\text {ges }}$ wird als Summe der Leitwerte für laminare und molekulare Strömung (in der Rohrleitung) angegeben. ${ }^{49}$

$$
L_{\mathrm{ges}}=L_{\mathrm{lam}}+L_{\mathrm{mol}}=\frac{\pi \cdot d^{4}}{128 \cdot \eta \cdot l} \cdot \bar{p}+\frac{\pi \cdot \bar{c} \cdot d^{3}}{12 \cdot l}
$$

Hierbei ist $d$ der Durchmesser und $l$ die Länge der Rohrleitung, $\eta$ die Viskosität des zu fördernden Gases, $\bar{p}$ der mittlere Druck in der Leitung und $\bar{c}$ die mittlere thermische Geschwindigkeit. Die letzten drei Größen sowie der Durchmesser der Leitung haben sich bei der neuen Kammer gegenüber der alten nicht geändert. Die Länge der Rohrleitung hat sich allerdings vervierfacht, wodurch sich der Leitwert verkleinert. Ein Knick in der Rohrleitung führt ebenfalls zu einer Verringerung des Leitwertes, da sich hierdurch ein zusätzlicher Strömungswiderstand ergibt. (In der oben angegebenen Formel können Knicke durch die Annahme einer größeren effektiven Länge berücksichtigt werden.) ${ }^{50}$ Die Anzahl an Umlenkungen in der Rohrleitung wurde von drei auf zwei verringert, wodurch der Effekt der längeren Rohrleitung abgeschwächt wird. Ein weiterer Unterschied besteht darin, dass die Abpumprichtung in der neuen Kammer orthogonal zur Expansionsrichtung und nicht in Strömungsrichtung wie in der alten Kammer ist. Der 
Effekt auf den Leitwert ist nicht eindeutig ermittelbar, vermutlich ergibt sich aber eine weitere Verringerung des Leitwertes, da auch ein Absaugen senkrecht zur Expansionsrichtung eine Art „Knick“ ergibt. Die Leitwertänderung kann den um $15 \%$ höheren Hintergrunddruck erklären.

Die erforderlichen Optikkomponenten wurden analog zum vorherigen Aufbau wieder verwendet. Die Signalqualität wurde mittels einer Jetexpansion von Luft anhand der Stickstoffschwingung, die als interner Standard für die Justierung genutzt wird, er-

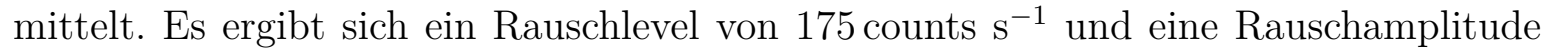
von 13 counts s ${ }^{-1}$ somit also vergleichbare Werte zum vorherigen Aufbau. Dementsprechend ist die Unterdrückung von Reflexionen durch den schwarzen Kunstschmiedelack der Edelstahlkammer ähnlich gut wie durch die schwarze Eloxalschicht der Aluminiumkammer.

Die Höhe des Q-Zweigs der Stickstoffschwingung ist um ca. $10 \%$ geringer als der durchschnittliche Wert, der für die Aluminiumkammer ermittelt wurde. Hier kann durch weitere Justierung beispielsweise der Ausrichtung des Monochromators noch eine Verbesserung erzielt werden, für Standardmessungen ist die Signalstärke aber vollkommen hinreichend.

Für den heizbaren Teil der Apparatur wurde ein neues Design des Sättigers entworfen. Der Sättiger besteht aus einer Außenhülle aus Edelstahl, die mit Heizbändern (HSS450, HORST GmbH) versehen ist, und einem Messinginnenteil, das auf der Außenseite eine Wendelstruktur besitzt. Das Trägergas wird über die Wendelstruktur nach unten geleitet und so vorgewärmt. Es tritt an der Unterseite des Sättigerinnenteils durch eine Fritte in die mit dem Analyten befüllte Innenkammer des Sättigers. Das SubstanzTrägergasgemisch wird dann über geheizte Schlauchleitungen in die Vakuumkammer befördert. Außerdem ist der Sättiger mehr als doppelt so lang wie der Sättiger der ersten Generation. ${ }^{11}$ Hierdurch soll verhindert werden, dass die Substanz in Tröpfchenform aus dem Sättiger herausgetragen wird, ein Problem das bei dem ersten Metallsättiger nur durch den Einsatz von Glaswolle abgemildert werden konnte. ${ }^{11}$ Des Weiteren wurde ein 4-Wege Kugelhahn mit einer X-Bohrung (Hydac Accessories GmbH 4/2 Wege Kugelhahn), der mit einer Heizung ( $4 \times$ Hochleistungs-Heizpatrone Typ 654, $\varnothing=6.5 \mathrm{~mm}$, Länge $40 \mathrm{~mm}, 100 \mathrm{~W}$, HS-Heizelemente, Heinz Stegmeier GmbH) versehen ist, eingesetzt. Hierdurch kann der Sättiger ohne aufwendige Umbaumaßnahmen umgangen werden. Eine Abbildung der beiden Bauteile befindet sich im Anhang (Abb. A.4.2). Allerdings wiesen einige Schlauchverbindungen gravierende Undichtigkeiten auf, die bis zum Ende dieser Arbeit noch nicht beseitigt werden konnten, so dass ein abschließender Test des neuen Sättigers ausblieb. 


\subsection{Lineare Raman-Spektroskopie gekoppelt mit IR-Laseranregung}

In der vorliegenden Arbeit wurde die in Kapitel 3.2 beschriebene Apparatur mit einer IR-Laseranregung gekoppelt. IR-Laseranregung mithilfe von OPO-Lasern wird bereits angewendet zur Untersuchung von Molekülen oder Molekülaggregaten in Heliumnanotröpfchen ${ }^{51,52}$ oder in Überschallexpansionen ${ }^{53}$ mittels IR-Photodissoziationsspektroskopie. Hierbei wird die IR-Laseranregung allerdings mit Massenspektrometrie gekoppelt. Außerdem werden bei dieser Technik meist gepulste Laser mit hoher Spitzenleistung oder besonders schmalbandige cw-Laser eingesetzt. Mittels eines IR-Lasers können selektiv Molekülschwingungen angeregt werden; die Folgen dieser Anregung sollen in der vorliegenden Arbeit mittels spontaner Raman-Streuung beobachtet werden.

Nach der IR-Anregung sind mehrere Prozesse möglich. Im Falle einer harmonischen Schwingung zeigt sich die IR-Anregung im Raman-Spektrum durch Zunahme des StokesRaman-Signals und gleichzeitige Zunahme des Anti-Stokes-Raman-Signals im Vergleich zum Raman-Spektrum ohne IR-Laseranregung. Die Zunahme des Stokes-Signals erfolgt aufgrund des größeren Übergangsmoments bei Übergängen aus schwingungsangeregten Zuständen, während die Zunahme des Anti-Stokes-Signals aufgrund der höheren Besetzung des Ausgangsniveaus erfolgt. (Siehe auch Kapitel 2.2 und Ref. 26.) Im anharmonischen Fall würde das Stokes-Signal abnehmen, während das Anti-Stokes-Signal zunimmt. Zusätzlich müssen im anharmonischen Fall noch Kopplungen zwischen den verschiedenen Schwingungen berücksichtigt werden, hier kann eine Energieumverteilung (IVR) stattfinden. ${ }^{13}$ Mittels linearer Raman-Spektroskopie kann zwar keine zeitaufgelöste Messung der Umverteilung erfolgen, es können aber Veränderungen von Signalintensitäten und Schwingungswellenzahlen beobachtet werden, die Hinweise darauf geben können, welche Schwingungen besonders stark aneinander gekoppelt sind. Der Ansatz die zeitabhängige Umverteilung in der Frequenzdomäne zu beobachten existiert bereits in der hochaufgelösten IR-Spektroskopie, wo aus der Intensitätsverteilung von Rotationsschwingungsübergängen Rückschlüsse auf die Energielokalisierung in einer Mode gezogen werden. ${ }^{54,55}$

Des Weiteren könnten durch die IR-Anregung Isomerisierungsbarrieren überwunden werden. Als Folge würde sich die Intensität von Banden, die zu weniger stabilen Isomeren gehören, erhöhen.

Bei schwach gebundenen Molekülkomplexen kann die Anregung von intramolekularen Schwingungen zur Destabilisierung der schwachen intermolekularen Bindungen führen, so dass es zum Bindungsbruch kommt. ${ }^{56}$ Bei acyclischen Komplexen kommt es dann zu einer einfachen Dissoziation, während bei cyclischen Aggregaten auch die Bildung von offenkettigen Strukturen bei gleichbleibender Anzahl an Monomereinheiten 
im Cluster möglich ist. Erste Hinweise auf solche Strukturen wurden bereits in warmen Expansionen mittels IR-Spektroskopie gefunden. ${ }^{57,58}$ Dieser Effekt würde sich im Raman-Spektrum ebenfalls als Abnahme des Clustersignals zeigen.

Von besonderem Interesse sind außerdem Komplexe mit Edelgasen. Beispielsweise wurden für fluorierte Alkohole sukzessive Umhüllungen mit Argon beobachtet. ${ }^{59}$ Mithilfe der IR-Anregung könnten die Argon-Atome abgedampft und möglicherweise auch verschiedene Umhüllungsstufen präpariert werden.

Für die verschiedenen Möglichkeiten ergeben sich unterschiedliche Anforderungen an die zu untersuchenden Moleküle und Molekülaggregate. Grundsätzlich sollten die Analyten eine stark IR-aktive Schwingungsbande im Bereich der Emission des verwendeten Lasers, in diesem Fall zwischen 3125 und $4000 \mathrm{~cm}^{-1}$, aufweisen. Ebenso sollte die zu untersuchende Schwingung, die von der IR-angeregten Schwingung verschieden sein kann, für erste Testexperimente eine starke Raman-Aktivität aufweisen, da so ein gutes Signal-zu-Rausch-Verhältnis erhalten wird. Für die Dissoziation von Clustern muss zudem die Dissoziationsenergie des Komplexes mit der Energie eines Photons überwunden werden können.

Als geeignete Systeme wurden zunächst aliphatische kurzkettige Alkohole ausgewählt. Für Ar-Umhüllungs- und Isomerisierungsexperimente eignen sich fluorierte Alkohole. ${ }^{17,60}$

\subsubsection{OPO-Laser: Eigenschaften laut Hersteller}

Zur IR-Anregung von Molekülkomplexen wurde ein durchstimmbarer IR-Laser (Optisch parametrischer Oszillator, OPO) der Firma Lockheed Martin Aculight (Argos Model 2400-BB-30(B)) verwendet, der laut Hersteller die in Tabelle 3.1 angegebenen Spezifikationen besitzt. ${ }^{43}$

\begin{tabular}{llll}
\hline & Pump & Signal & Idler \\
\hline$P_{\max } / \mathrm{W}$ & 32 & 15 & 9 \\
$\lambda_{\mathrm{IR}} / \mu \mathrm{m}$ & 1.064 & $1.6-1.85$ & $2.5-3.2$ \\
$\lambda_{\mathrm{VIS}} ; P$ & $500-700 \mathrm{~nm} ;<1 \mathrm{~mW}$ & $500-700 \mathrm{~nm} ;<1 \mathrm{~mW}$ & $500-700 \mathrm{~nm} ;<1 \mathrm{~mW}$ \\
\hline
\end{tabular}

Tabelle 3.1: Leistungs- und Wellenlängenspezifikationen des OPO-Lasers laut Hersteller. ${ }^{43}$

Zur Wellenlängeneinstellung können verschiedene Wege genutzt werden. Wichtigste Möglichkeit ist die Verstellung der Kristallposition. Durch Translation des Kristalls mittels einer Schraube trifft der Pumplaser auf verschiedene Bereiche des PPLN-Kristalls und somit auf verschiedene Abstände der gepolten Bereiche, so dass unterschiedliche Wellenlängen die Bedingung für die Quasiphasenanpassung erfüllen. Außerdem kann 
die Temperatur des Kristalls $\left(\vartheta_{\text {Crystal }}\right)$ variiert werden, was ebenfalls zu einer Veränderung der Wellenlänge führt. Laut Hersteller gelten für die Verstellung der Idlerwellenlänge $\lambda_{\mathrm{I}}$ die groben Richtwerte von $23.2 \mathrm{~nm} /$ Schraubendrehung und $-2.4 \mathrm{~nm} / \mathrm{K}{ }^{43}$

Außerdem verfügt der Laser über ein Etalon, das eine zusätzliche Frequenzstabilisierung bietet. Durch Variation des Etalonwinkels wird die Abstimmung der Oszillationswellenlänge des Etalons auf die maximal verstärkte Wellenlänge des Kristalls erreicht. ${ }^{43}$ Durch Variation des Etalonwinkels verändert sich die Frequenz um bis zu $800 \mathrm{GHz}$, was einer Wellenzahländerung von ca. $27 \mathrm{~cm}^{-1}$ entspricht.

Die Einstellung der Wellenlänge in diesem Modus ist sehr zeitraubend: iterativ muss die Kristallposition eingestellt, dann die Wellenlänge bestimmt und der Etalonwinkel auf maximale Leistung optimiert werden, dann muss erneut die Wellenlänge bestimmt werden.

Douberly et al. lösten dieses Problem indem sie die Optimierung automatisierten. ${ }^{52}$ Der technische Aufwand ist für den in Göttingen verwendeten Aufbau allerdings nicht gerechtfertigt, da hier kein Scan der Wellenlänge notwendig ist, sondern vielmehr nach der Einstellung die gewählte Wellenlänge über einen längeren Zeitraum von Stunden bis hin zu Wochen beibehalten wird. Zudem bietet der Betrieb mit Etalon außer der Frequenzstabilisierung keine großen Vorteile gegenüber dem Betrieb ohne, so dass das Etalon später entfernt werden konnte. Bei hohen Idlerfrequenzen kann das Etalon allerdings relevant sein, da hier mehrere Moden resonant sein können und mittels des Etalons dann eine Mode ausgewählt werden kann. ${ }^{52}$ Solange dieses Verhalten aber nicht beobachtet wird, kann auf das Etalon verzichtet werden.

Die angegebenen Maximalwerte können nicht in allen Bereichen realisiert werden. Als weitaus wichtigere Spezifikationen gelten daher die garantierten Mindestleistungen von Signal- und Idlerstrahl. Sie belaufen sich auf $2 \mathrm{~W}$ für den Idler- und $4 \mathrm{~W}$ für den Signalausgang. ${ }^{61}$ Leistungseinbußen bestehen vor allem im Bereich um $2830 \mathrm{~nm}\left(3534 \mathrm{~cm}^{-1}\right)$, da hier OH-Absorptionen im MgO:PPLN-Kristall auftreten. Außerdem variiert die Ausgangsleistung im Bereich zwischen 2400 und $2700 \mathrm{~nm}$ aufgrund von Wasserabsorptionen in der Kavität, was aber durch eine Trockenluftspülung abgeschwächt werden kann. ${ }^{43}$

Neben dem infraroten Licht emittieren alle drei Ausgänge parasitär auch im sichtbaren Bereich mit Leistungen im sub-mW Bereich und zwar einerseits bei $532 \mathrm{~nm}$ (frequenzverdoppelte Pumpwellenlänge) und andererseits im roten Bereich bei verschiedenen Wellenlängen in Abhängigkeit von der Kristallposition (Summenfrequenz von Signalund Pumpstrahl). Der Strahldurchmesser $\left(1 / \mathrm{e}^{2}-\right.$ Wert $)$ des Idlers beträgt am Laserkopfausgang je nach Wellenlänge ca. 3-4 mm. Von hier aus pflanzt sich der Laserstrahl gemäß Gaußoptik fort. Alle drei Laserstrahlen sind linear polarisiert mit horizontaler Richtung des elektrischen Feldes. ${ }^{62}$ 


\subsubsection{OPO-Laser: Testmessungen}

Als erster Test wurde die Leistung des Idler-, Signal- und Pumpstrahls bei den voreingestellten Bedingungen $\left(\vartheta_{\text {Crystal }}=52{ }^{\circ} \mathrm{C}, \vartheta_{\text {Block }}=38.5^{\circ} \mathrm{C}, \lambda=2942.0 \mathrm{~nm}\right)$ unter Verwendung desselben Powermeters ermittelt. In Abbildung 3.3 ist der Verlauf der Leistung in Abhängigkeit des Quellstroms des Pumplasers (angegeben in \% des Quellstroms) gezeigt. Die Pumpleistung durchläuft bei $60 \%$ ein Maximum. Hier setzt die Konversion der Pumpstrahlung in Idler und Signal ein. Bis zu diesem Wert besitzen Idler und Signal keine signifikante Intensität, danach steigen sie kontinuierlich und näherungsweise linear mit steigendem Quellstrom an. Außerdem wird ab diesem Wert neben dem grünen Licht auch noch rotes Licht emittiert. Als Endwerte bei voller Pumplaserleistung ergeben sich $5 \mathrm{~W}$ Idler-, $11 \mathrm{~W}$ Signal- und $5.5 \mathrm{~W}$ Pumpleistung. In Abbildung 3.3 ist außerdem die Gesamtleistung von S, P und I gezeigt (unausgefüllte schwarze Kreise); der Anstieg verläuft näherungsweise linear.

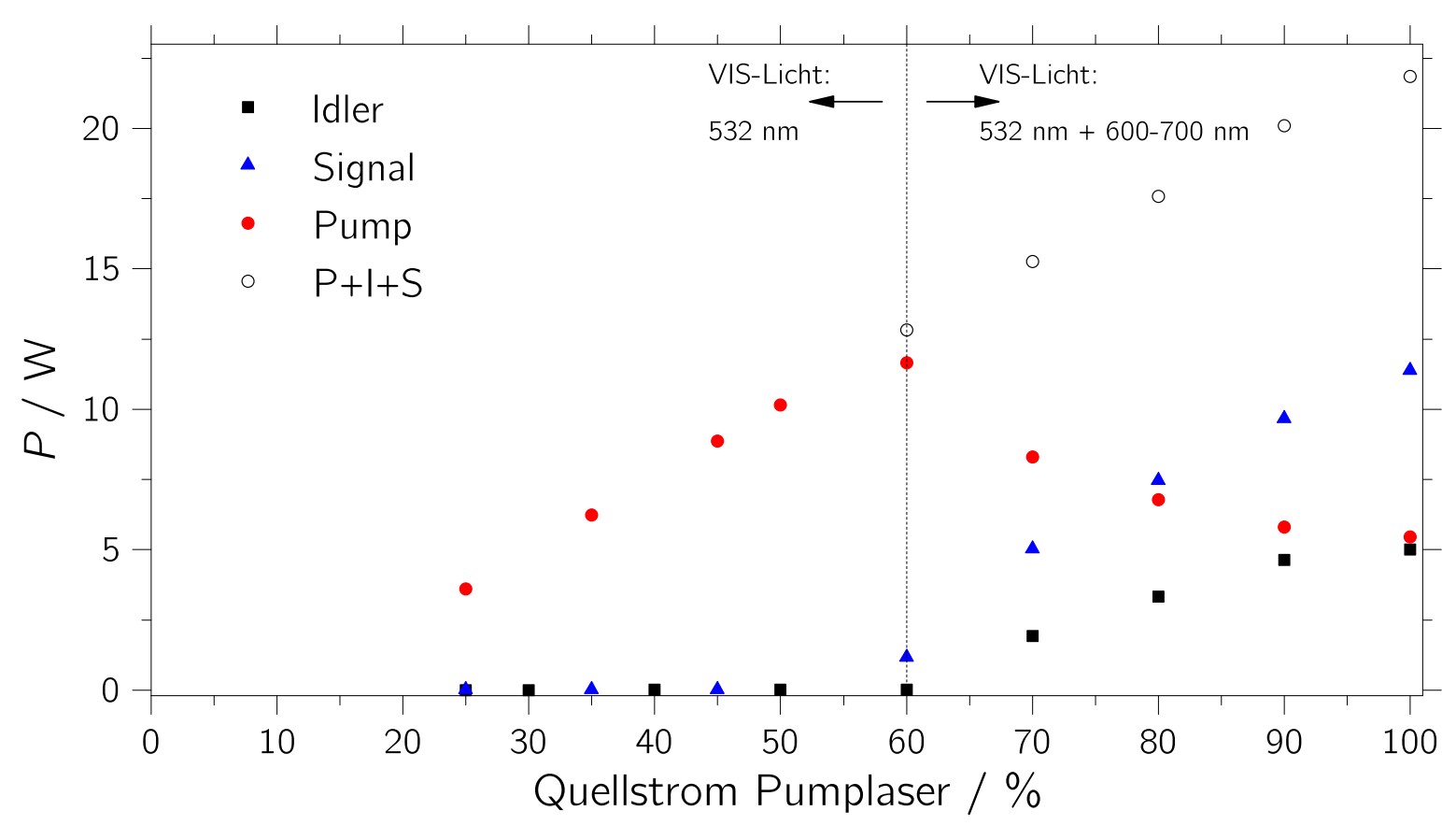

Abbildung 3.3: Leistungsverlauf in Abhängigkeit vom Quellstrom des Pumplasers. Bei Anlieferung gegebene Einstellungen $\vartheta_{\text {Crystal }}=52.0^{\circ} \mathrm{C}, \vartheta_{\text {Block }}=38.5^{\circ} \mathrm{C}, \lambda_{\mathrm{I}}=2942.0 \mathrm{~nm}$.

Des Weiteren wurde die Leistungsstabilität überprüft. Hierzu wurde eine Langzeitleistungsmessung (Abb. 3.4) durchgeführt. Die Leistung wurde über einen Zeitraum von 3 Stunden erfasst. In der ersten halben Stunde wurde sie in Abständen von 5 Minuten verfolgt; danach in 10 Minuten Abständen erfasst. Es zeigt sich, dass die Leistung nach der ersten Stunde um 5\% abfällt, danach jedoch stabil ist. Das kann daran liegen, 
dass der OPO eine Aufwärmphase durchläuft, die nach einer Stunde abgeschlossen ist. In jedem Fall ist ein Leistungseinbruch von $5 \%$ vernachlässigbar klein und stellt für spätere Messungen kein signifikantes Problem dar.

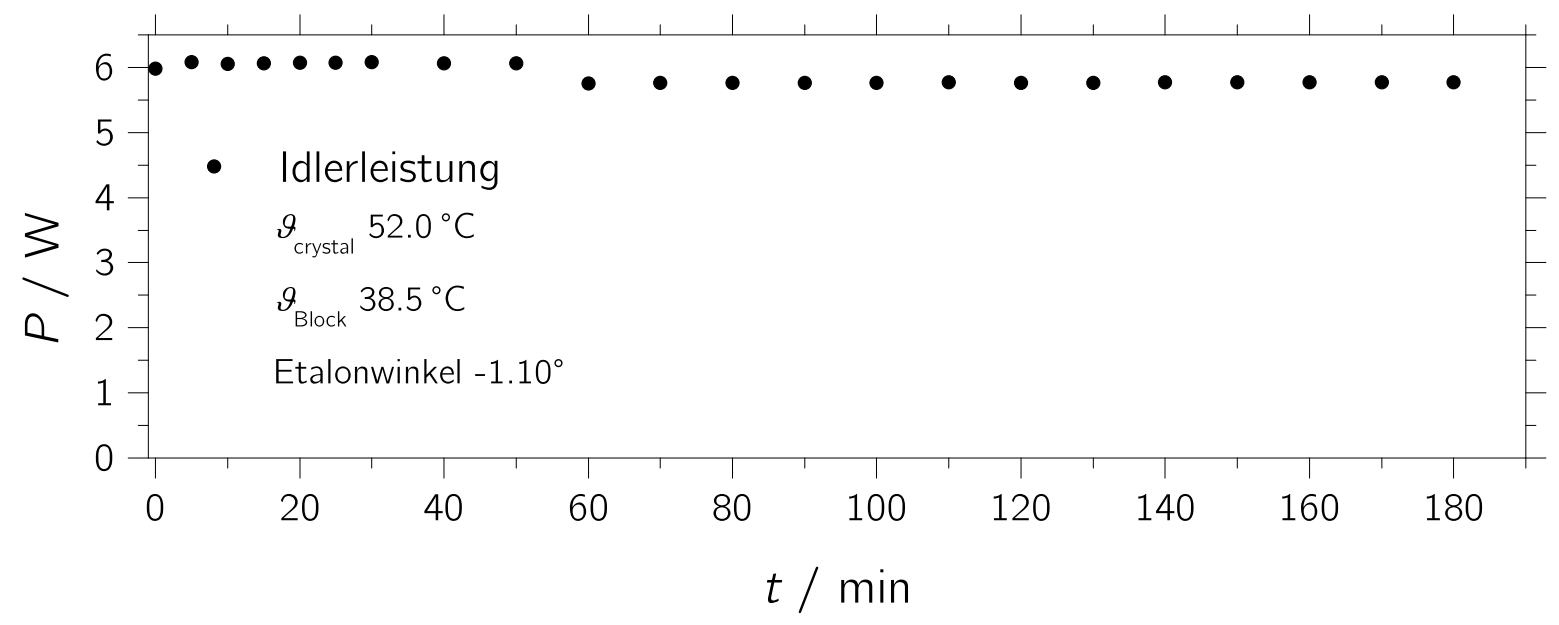

Abbildung 3.4: Erfassung der Idlerleistung über einen Zeitraum von $3 \mathrm{~h}$.

Neben der Stabilität und dem Leistungsverlauf bei verschiedenen Quellströmen ist besonders die Leistung in Abhängigkeit von der Kristallposition und somit von der Wellenlänge von Interesse. Zunächst wurde die Leistung ohne eine Verstellung des Etalonwinkels gemessen. In Abbildung 3.5 ist die Leistung gegen die Kristallposition ausgedrückt als Schraubenumdrehung, wobei positive Werte eine Rechtsdrehung bezeichnen, gezeigt. Da die Wellenzahl an der Stelle Umdrehung $=0$ mit $3400 \mathrm{~cm}^{-1}$ bekannt war, konnte außerdem mithilfe der Herstellerangaben die ungefähre Wellenzahl berechnet werden. Sie ist in der oberen $x$-Achse angegeben. Abbildung 3.5 zeigt eine Oszillation der Leistung mit der Wellenzahl, da das Etalon nur bestimmte Wellenlängen verstärkt. Diese Oszillation konnte in mehreren Messungen an verschiedenen Versuchstagen reproduziert werden. In der Abbildung ist außerdem die theoretische Leistung unter der Annahme, dass für jede Wellenlänge die gleiche Anzahl Photonen emittiert wird, eingezeichnet. Als Referenzpunkt wurde das Maximum bei $3461 \mathrm{~cm}^{-1}$ verwendet. Zwischen 3100 und $3500 \mathrm{~cm}^{-1}$ ergibt sich eine regelmäßige Oszillation der Leistung und die Leistungsmaxima folgen dem theoretisch berechneten Trend. Um $3535 \mathrm{~cm}^{-1}$ sind Unregelmäßigkeiten mit um bis zu $50 \%$ geringerer Leistung zu erkennen. Der Bereich des Leistungseinbruchs stimmt mit dem vom Hersteller angegebenen Bereich der $\mathrm{OH}-$ Absorption im Kristall überein. Weiterhin sind geringere Leistungen zwischen 3650 und $3750 \mathrm{~cm}^{-1} \mathrm{zu}$ verzeichnen. Hier befindet sich der Bereich der Absorptionen durch Wasserdampf in der Kavität.

Die OH-Absorption im Kristall kann nicht durch Optimierung des experimentellen Aufbaus (beispielsweise einer Luftspülung) verringert werden. Daher ist dieser Bereich kritisch für die Angabe der garantierten Mindestleistung. In diesem Bereich wurde 


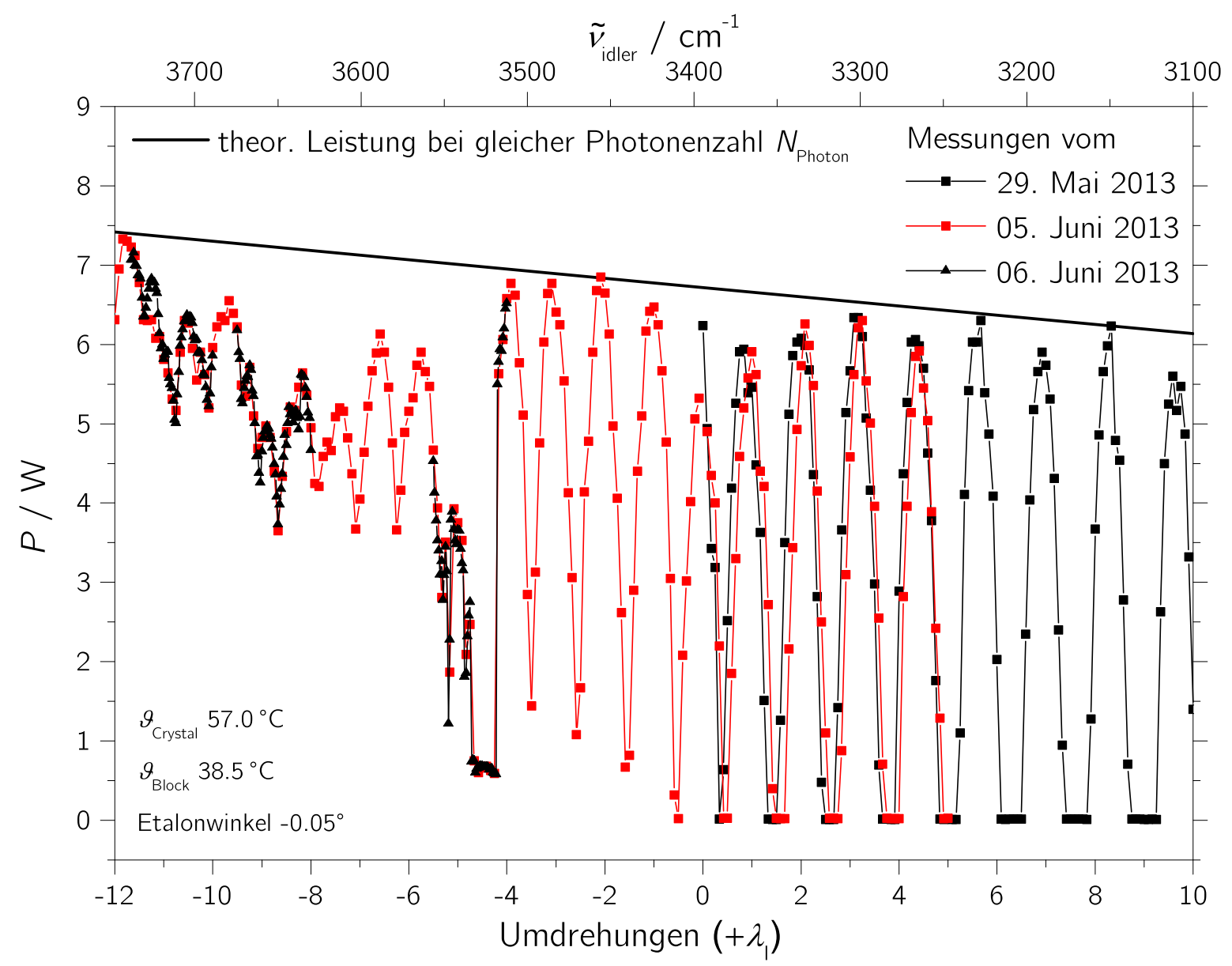

Abbildung 3.5: Leistungsentwicklung mit Veränderung der Kristallposition bei konstantem Etalonwinkel. Die in der oberen $x$-Achse angegebenen Wellenzahlwerte wurden aus den Herstellerangaben und dem Referenzpunkt Umdrehung $=0$ berechnet. Es handelt sich also um ungefähre Werte. Unregelmäßigkeiten in der Oszillation der Leistung weisen auf Leistungseinbrüche durch Absorptionen im Kristall oder in der Kavität und auf dem Weg zum Detektor hin. Die Messergebnisse konnten an verschiedenen Messtagen reproduziert werden. Die Maxima der Leistung folgen mehrheitlich dem theoretischen Trend. 
dementsprechend die maximal erreichbare Leistung durch Optimierung des Etalonwinkels untersucht. Abbildung 3.6 zeigt eine Auftragung der Leistung gegen den Etalonwinkel. Es ergeben sich zwei Leistungsmaxima, ein lokales Maximum bei $-1.6^{\circ}$ mit $2.53 \mathrm{~W}$ und ein globales bei $1.3^{\circ}$ mit $3.06 \mathrm{~W}$. Beide Maxima liegen aber oberhalb der garantierten Mindestleistung.

Auch die Temperaturabhängigkeit der Leistung wurde bei festgehaltenem Etalonwinkel bestimmt, die in Abbildung A.4.3 im Anhang gezeigt ist. Es ergibt sich erneut eine Leistungsoszillation. Aus den Abständen der Leistungsminima konnte eine Idlerwellenlängenänderung von $-2.4 \mathrm{~nm} / \mathrm{K}$ ermittelt werden, die mit den Angaben des Herstellers übereinstimmt.

Die weiteren Tests und die Verwendung des OPO-Lasers im Experiment erfolgten aufgrund der oben genannten Gründe nach Ausbau des Etalons.

Um die Einstellung der Wellenlänge komfortabler zu gestalten wurde ein elektronisches Zählwerk eingebaut, das über einen Inbusschlüssel mit der Stellschraube verbunden ist. Eine Schraubenumdrehung entspricht einer Zählerstandsänderung von 500. Zur Grobeinstellung der Wellenlänge ohne Ermittlung der genauen Wellenzahl der Idlerstrahlung, wurde eine Kalibrierkurve für eine Kristalltemperatur von $57^{\circ} \mathrm{C}$ aufgenommen. Der Zählerstand wurde in Schritten von 250 variiert und jeweils die Idlerwellenzahl mittels FTIR-Spektroskopie bestimmt. Abbildung 3.7 zeigt die Auftragung des Zählerstands gegen die Idlerwellenzahl. Es wurde ein Polynom dritten Grades an die Kurve angepasst. Mit diesem kann nun berechnet werden welcher Zählerstand für eine bestimmte Idlerwellenzahl eingestellt werden muss. Die Kurve besitzt lediglich eine Genauigkeit von $6 \mathrm{~cm}^{-1}$.

Nach der Grobeinstellung mithilfe der Kalibrierkurve wird die Wellenzahl bestimmt und gegebenenfalls der Zählerstand weiter angepasst. Danach wird die Ausgangsleistung bestimmt. Ist diese zu gering, wird eine Anpassung der Kristalltemperatur vorgenommen und ggf. noch einmal die Kristallposition verändert bis die gewünschte Wellenzahl mit der gewünschten Ausgangsleistung erreicht ist.

Diese Vorgehensweise ist notwendig, da der Laser bei einer bestimmten Kristalltemperatur verschiedene Moden besitzt. Der Modenabstand ist für den Bereich um $4000 \mathrm{~cm}^{-1}$ $6 \mathrm{~cm}^{-1}$, für den Bereich um $3125 \mathrm{~cm}^{-1} 1-2 \mathrm{~cm}^{-1}$.

Dementsprechend sind bestimmte Wellenlängen nur durch eine Anpassung der Temperatur zugänglich. Abbildung A.4.4 im Anhang zeigt die Leistungsschwankung im Bereich um $4000 \mathrm{~cm}^{-1}$ beispielhaft für drei Lasermoden bei einer Kristalltemperatur von $57^{\circ} \mathrm{C}$. Die Kristallposition wurde hier maximal um einen Zählerstand von \pm 50 variiert. Es zeigen sich Abweichungen von bis zu 1.2 W. Die Leistungsschwankung könnte durch eine geringere Konversionseffizienz bedingt sein, die möglicherweise auftreten, wenn der gepolte Bereich nicht zentral vom Pumplaser getroffen wird. 


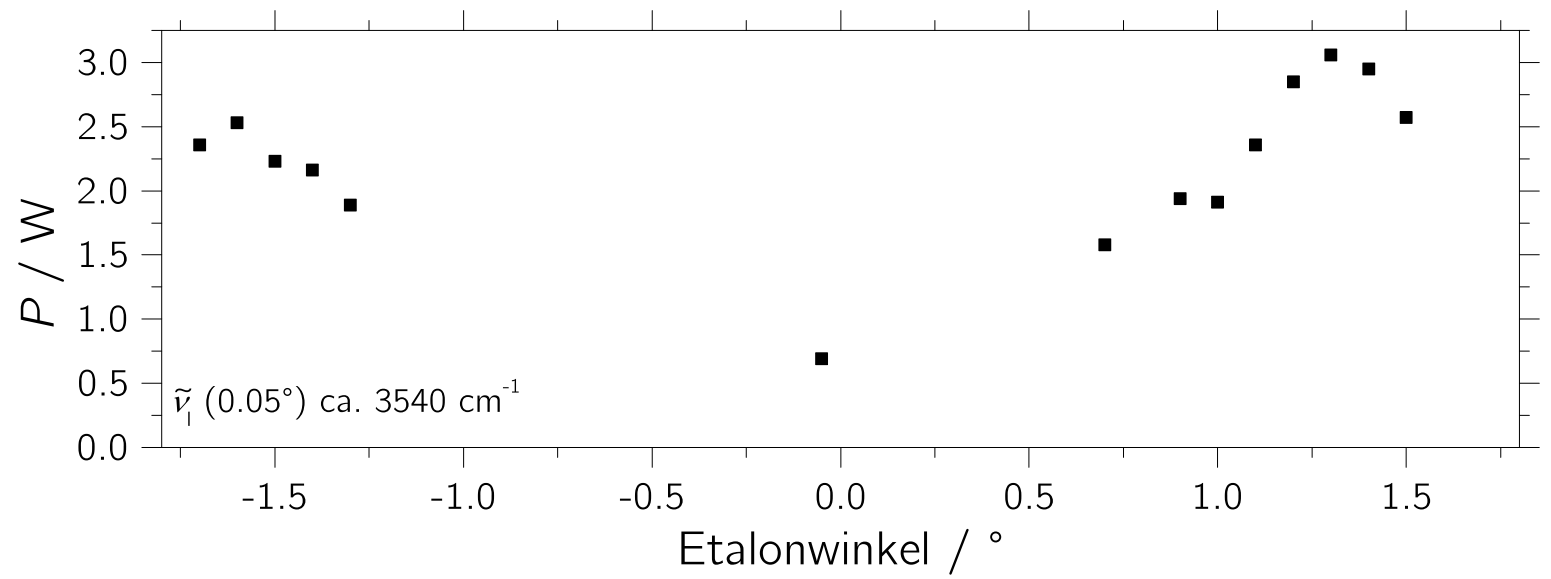

Abbildung 3.6: Abhängikeit der Leistung vom Etalonwinkel im Bereich der OH-Absorption im PPLN-Kristall.

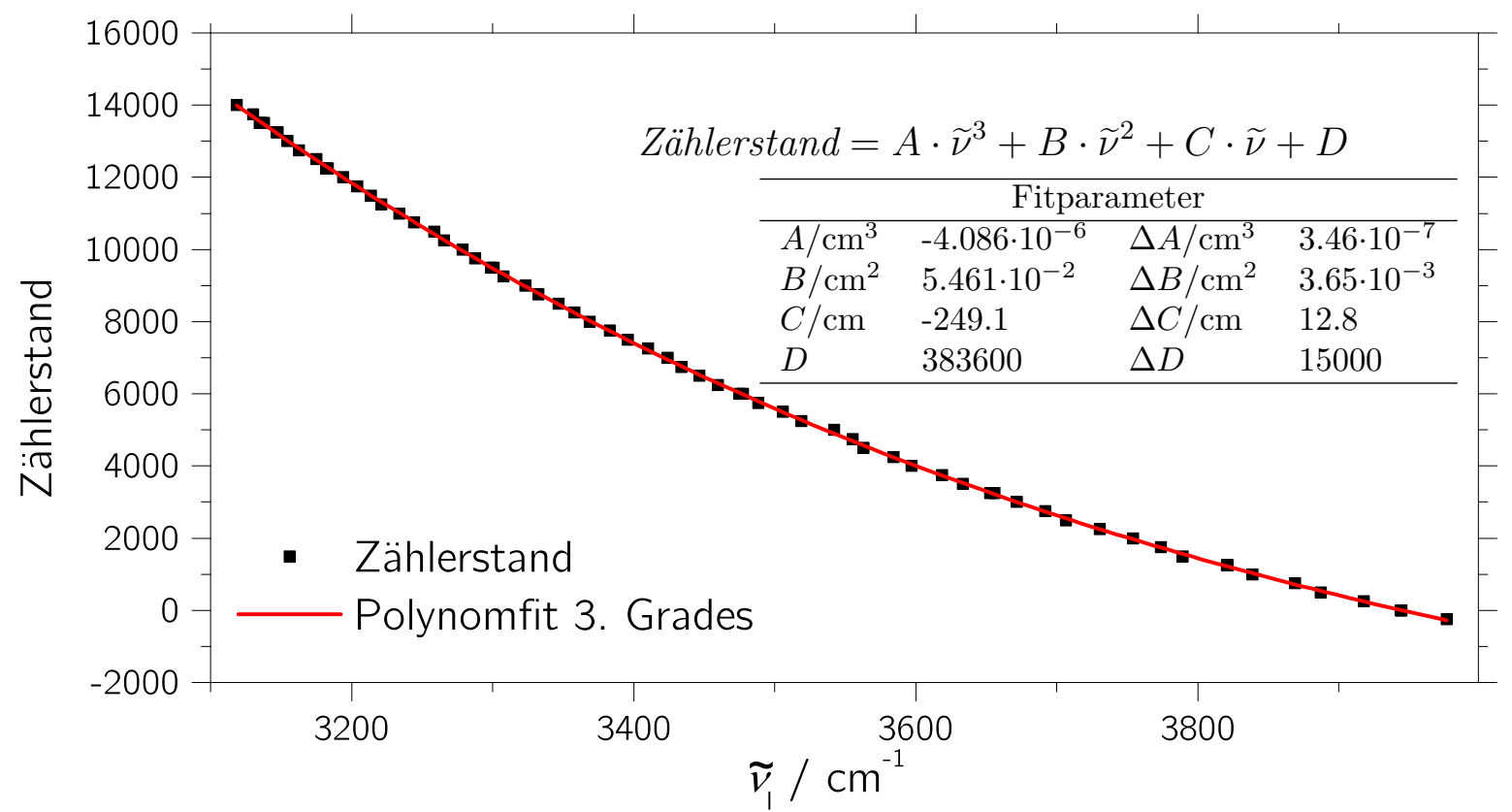

Abbildung 3.7: Kalibrierkurve zur Ermittlung der Kristallposition (ausgedrückt durch den Zählerstand), die für eine gewünschte Idlerwellenzahl eingestellt werden muss. Gültig für: $\vartheta_{\text {Crystal }}=57.0^{\circ} \mathrm{C}, \vartheta_{\text {Block }}=40.5^{\circ} \mathrm{C}$. 


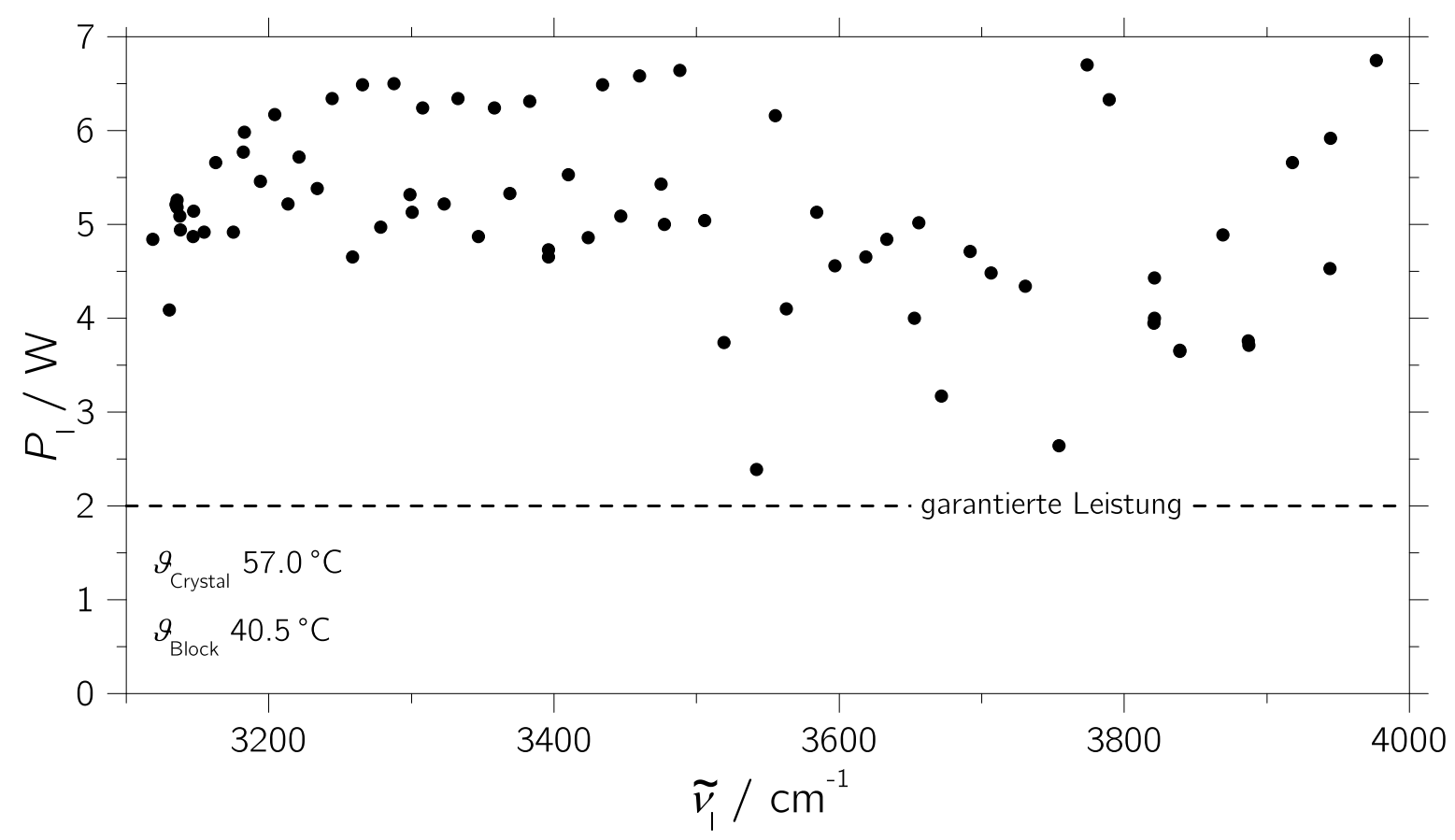

Abbildung 3.8: Leistungswerte des OPO-Lasers in Abhängigkeit von der Idlerwellenzahl nach Ausbau des Etalons.

Es wurde außerdem erneut die Leistung in Abhängigkeit von der Wellenzahl bestimmt. Hier zeigte sich keine systematische Entwicklung der Leistung mit der Wellenlänge. Hierbei wurde allerdings keine weitere Leistungsoptimierung durchgeführt, so dass die maximal erreichbaren Leistungswerte höher liegen als in Abbildung 3.8 gezeigt. Zwischen 3100 und $3500 \mathrm{~cm}^{-1}$ können Leistungswerte zwischen 4 und $7 \mathrm{~W}$ erreicht werden. Zwischen 3500 und $4000 \mathrm{~cm}^{-1}$ sind die Leistungswerte im Durchschnitt niedriger. Hier hängt die erreichte Leistung maßgeblich davon ab, ob die eingestellte Wellenzahl mit einem Rotationsschwingungsübergang von Wasser zusammenfällt.

Die Polarisationsrichtung des Idlerstrahls wurde mittels eines Siliziumfensters bestimmt. Das Fenster wurde in den Strahlengang gebracht und die transmittierte und reflektierte Strahlung in Abhängigkeit des Winkels zwischen Fenster und Ausbreitungsrichtung des Lasers mittels zweier Leistungsmessgeräte ermittelt. Es konnte bestätigt werden, dass die Idlerstrahlung horizontal polarisiert ist. 


\subsubsection{Unterdrückung der OPO-Strahlung zwischen 400 und $700 \mathrm{~nm}$}

Ein erhebliches Problem für ein IR-Laser-Raman-Experiment stellt das vom OPO emittierte sichtbare Licht dar. Zum einen kann das im roten Bereich liegende Licht die zu messenden Stokes-Banden durch Reflexionen bspw. an der Düse überlagern, zum anderen kann auch das grüne Licht, das dieselbe Wellenlänge wie der Raman-Anregungslaser besitzt, stören, da die Intensität der zu entfernenden Rayleighstreuung um den Betrag der vom OPO emittierten Strahlung erhöht ist. Andererseits ist die sichtbare Strahlung aber zur Justierung sehr nützlich, so dass ein „An- und Abschalten“ der Emission vorteilhaft ist. Aus diesem Grund wurde ein motorisierter Klapphalter (Newport Spectra Physics GmbH, Motorized Flipper Mount, Art.-No. 8892-K-M, $\varnothing=25.4$ mm, Wiederholgenauigkeit $<25 \mu \mathrm{rad}$ ) installiert, mit dem ein optisches Element mit großer Reproduzierbarkeit in den Strahlengang eingebracht und wieder hinaus gefahren werden kann. Zunächst wurde ein Siliziumfenster verwendet. Um Verluste zu minimieren wurde das Fenster im Brewsterwinkel eingesetzt, wobei es sich um einen mittleren Brewsterwinkel für den ansteuerbaren Frequenzbereich von ca. $75^{\circ}$ handelt. Aufgrund dieses Winkels erzeugt das Fenster aber einen Strahlversatz, daher wurde das Siliziumfenster später durch einen IR-Filter (Alkor Technologies, Ge-Substrat $\varnothing=25 \mathrm{~mm} \times 1 \mathrm{~mm}$, $4100-2500 \mathrm{~cm}^{-1}$, Transmission $>80 \%$ ) ersetzt, der im $90^{\circ}$-Winkel zum Strahlverlauf eingesetzt wurde. Der Filter führt aber im Gegensatz zum Siliziumfenster zu einem Verlust von bis zu $20 \%$ der Leistung.

\subsubsection{Idlerwellenlängenbestimmung mittels FTIR-Spektrometer}

Um die Idlerwellenlänge zu bestimmen wurde ein FTIR-Spektrometer der Firma Bruker (Vector 22, Bruker Optik GmbH, Ettlingen, Deutschland) modifiziert. Die Breitbandlichtquelle wurde entfernt und zwei Einkoppelspiegel eingesetzt. Der Laser muss zum Schutz des LADTGS-Detektors abgeschwächt werden. Hierzu wurde zunächst ein rückwandig polierter Silberspiegel (backside-polished protected silver mirror, Thorlabs GmbH, Art.-Nr.:PF10-03-P01P) verwendet. Dieser lässt ca. 0.01 \% der Strahlung durch und ist daher nur für kurze Weglängen zwischen OPO und Spektrometer geeignet. Bei längeren Wegen treten zu große Verluste durch Streuung oder Absorption in der Umgebungsluft auf, so dass die Signalstärke unterhalb der Detektorschwelle liegt. Daher wurde der Spiegel für die spätere Messroutine durch zwei Ni-beschichtete ZnSe-Filter (IR Reflective Neutral Density Filter, Thorlabs GmbH) ersetzt, so dass das Signal zuerst um zwei (OD 2.0) und dann um eine (OD 1.0) Größenordnung abgeschwächt wird. Insgesamt werden also noch ca. $0.1 \%$ der Ausgangsintensität der Laserstrahlung in das Spektrometer eingekoppelt. Nach Abschwächung wird die OPO-Strahlung zusammen mit der breitbandigen Strahlung einer Glühlampe über die zwei Einkoppelspiegel 


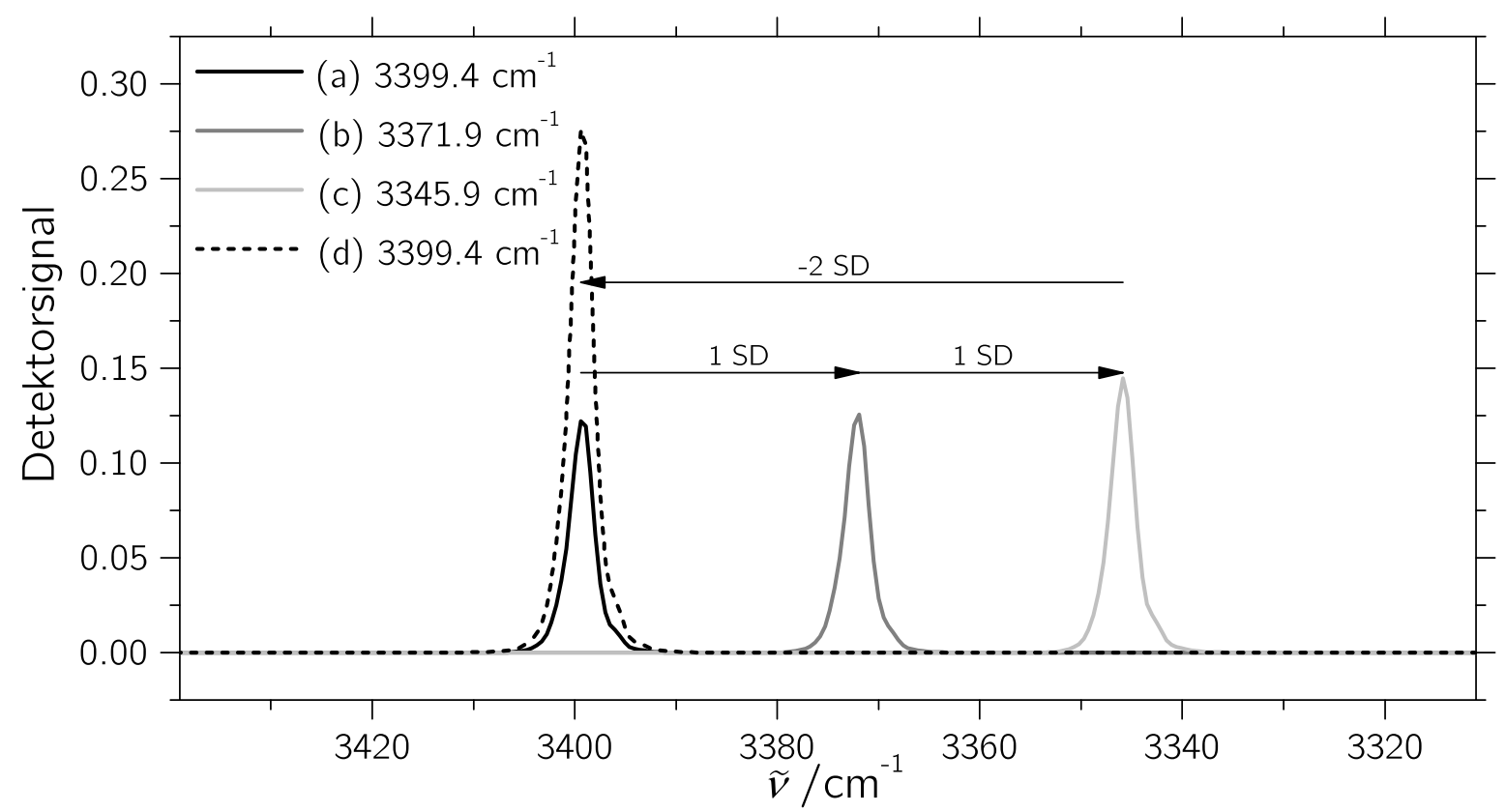

Abbildung 3.9: Beispiele von FTIR-Spektren zur Wellenlängenbestimmung des Idlers. Die Messreihenfolge sowie die Abstände der Spektren in Abhängigkeit von der Kristallposition sind durch Pfeile und die Anzahl der Schraubenumdrehungen (SD) angegeben.

in das Spektrometer gelenkt. Die zusätzliche Breitbandlichtquelle ist notwendig, da das Spektrometer die Nullposition des beweglichen Interferometerspiegels sonst nicht bestimmen kann.

Die Einstellungen des FTIR-Spektrometers zur Spektrenaufnahme befinden sich im Anhang (Tabelle A.4.2). Es wurden Einkanalspektren aufgenommen, daher die Angabe Detektorsignal für die Intensität anstelle der für basislinienkorrigierte Spektren üblichen Absorbanz.

Das OPO-Signal zeigt sich als gaußförmiges Profil im Spektrum. In Abbildung 3.9 sind Spektren für vier verschiedene Zählerstände aufgetragen, die Peakpositionen sind in der Legende angegeben. Die Reihenfolge der Spektrenaufnahme ist mit Pfeilen gekennzeichnet. Es wurde jeweils eine Schraubenumdrehung (SD) ausgeführt um von Spektrum (a) zu (b) und von Spektrum (b) zu (c) zu gelangen. Hierdurch konnte eine Wellenlängenänderung der Idlerstrahlung pro Schraubenumdrehung von 23-24 nm ermittelt werden. Diese stimmt gut mit den Herstellerangaben überein. Des Weiteren konnte die Reproduzierbarkeit der Schraubeneinstellung geprüft werden, indem nach Aufnahme Spektrum (c) um zwei Umdrehungen zurück auf den Ausgangswert gestellt wurde. In Spektrum (d) besitzt die OPO-Strahlung im Rahmen der Messgenauigkeit die gleiche Wellenzahl wie in Spektrum (a). Die Wellenlängenänderung pro Schraubenumdrehung konnte auch in weiteren Test bestätigt werden. Allerdings ist die Reproduzierbarkeit 


\begin{tabular}{cccc}
\hline Spektrum & Idlerwellenzahl $/ \mathrm{cm}^{-1}$ & FWHM $/ \mathrm{cm}^{-1}$ & $\begin{array}{c}\text { Spezifikation }^{61} \\
\text { FWHM } / \mathrm{cm}^{-1}\end{array}$ \\
\hline 20130924_OPO_2 & 3938 & 4.2 & $<6.7$ \\
20130909_OPO_16 & 3505 & 5.2 & $<6.7$ \\
20130924_OPO_28 & 3137 & 5.6 & $<6.7$ \\
\hline
\end{tabular}

Tabelle 3.2: Volle Halbwertsbreite der Idlerstrahlung exemplarisch für drei Wellenzahlwerte.

der Wellenzahlen besonders bei kleinen Verstellwegen der Schraube (= Zählerstandsänderung von $\leq \pm 50)$ nicht so präzise wie in dem oben gezeigten Beispiel. Es kann zu Abweichungen um bis zu $6 \mathrm{~cm}^{-1}$ kommen, da ein Sprung zur nächsten Lasermode erfolgt. Um diesen Effekt zu vermeiden empfiehlt es sich möglichst große Verfahrwege zu wählen.

In einigen FTIR-Spektren konnte neben der Idlerstrahlung gleichzeitig auch noch die Signalstrahlung beobachtet werden. Diese ist wahrscheinlich aufgrund von Reflexionen am Strahlfänger und am OPO-Kopf in den Idlerstrahlengang eingekoppelt worden. (Bei späteren Routineaufnahmen wurde ausschließlich die Idlerstrahlung beobachtet.) Sie zeigt sich als zusätzlicher Peak zwischen 5400 und $6250 \mathrm{~cm}^{-1}$. In Abbildung A.4.5 im Anhang sind beispielhaft vier FTIR-Spektren, die sowohl den Signal- als auch den Idlerbereich abdecken, gezeigt und in Tabelle A.4.1 sind die zugehörigen Wellenzahlen der Signal- und Idlerstrahlung aufgelistet. Als Plausibilitätsprüfung ist auch die Summe der beiden Werte angegeben. Sie ergibt sich für alle vier Spektren zu $9398 \mathrm{~cm}^{-1}$ und entspricht somit der Pumpwellenlänge von $1064 \mathrm{~nm}$.

Mithilfe der Software OPUS können nach der Spektrenaufnahme die genaue Position und die volle Halbwertsbreite des Idlers ermittelt werden. In Tabelle 3.2 sind die vollen Halbwertsbreiten für drei Beispielspektren im oberen, mittleren und unteren erreichbaren Wellenzahlbereich aufgelistet. Alle drei Werte liegen unterhalb der Herstellerangabe und die Halbwertsbreiten sinken für steigende Wellenzahlen. Hierbei muss allerdings beachtet werden, dass die Auflösung des FTIR-Spektrometers empfindlich von der Justierung abhängt. Bei Einkopplung der Strahlung abweichend vom idealen Winkel kommt es zu einer zusätzlichen Dämpfung des Signals. Dieser Effekt wird als Selbstapodisierung bezeichnet und zeigt sich im Spektrum durch verbreiterte Linien. ${ }^{63} \mathrm{Um}$ zu überprüfen, ob der Effekt einen signifikanten Einfluss auf die Spektren hat, wurden die Wasserrotationsschwingungsübergänge betrachtet. Einmal wurden sie mit der Glühlampe als Lichtquelle und offener Apertur gemessen und einmal mit dem Globar und einer Apertur von $3 \mathrm{~mm}$. Es konnte keine unterschiedliche Verbreiterung festgestellt werden, daher kann davon ausgegangen werden, dass der Effekt eine sehr kleine Auswirkung auf die Laserlinienbreite hat. Einen weiteren Einfluss auf die gemessene Linienbreite kann die Apodisierungsfunktion haben, weshalb aus den gemessenen Interferogrammen Spektren unter Nutzung dreier verschiedener Apodisierungsfunktio- 
nen (Boxcar, Norton-Beer, Medium und Blackman-Harris, 3 Term) erzeugt wurden. Die durch die Apodisierungsfunktion auftretende Verbreiterung des Lasersignals beläuft sich auf $0.1-0.3 \mathrm{~cm}^{-1}$ und ist somit vernachlässigbar. Die Linienbreiten werden also durch die Messmethode kaum verändert und sind daher als verlässliche Werte anzusehen.

Im Bereich der Rotationsschwingungsübergänge von Wasser zeigen sich Bandenformen im Spektrum, die vom Gaußprofil stark abweichen. Abbildung 3.10 zeigt eine Gegenüberstellung der Wasserbanden mit drei gemessenen Laserprofilen. Einbrüche in den OPO-Spektren stimmen mit den Positionen der Absorptionsbanden von Wasser überein, während sich Maxima bei in den Zwischenräumen zwischen den Banden ergeben. Da als Wellenzahl immer die höchste Intensität verwendet wurde und nicht die bei einer asymmetrischen Bande schwierig zu bestimmende Mitte der Bande (s. grau gestrichelte Linie in Abbildung 3.10), können die gemessenen Wellenzahlwerte im Bereich der Wasserabsorption stärker von den theoretisch erwarteten Werten abweichen. In diesem Wellenzahlbereich könnte eine höhere Leistung erzielt werden, indem durch eine Ummantelung des gesamten Strahlengangs außerhalb der Vakuumkammer (einschließlich des Laserkopfs) verbunden mit einer Trockenluftspülung die Absorption minimiert wird.

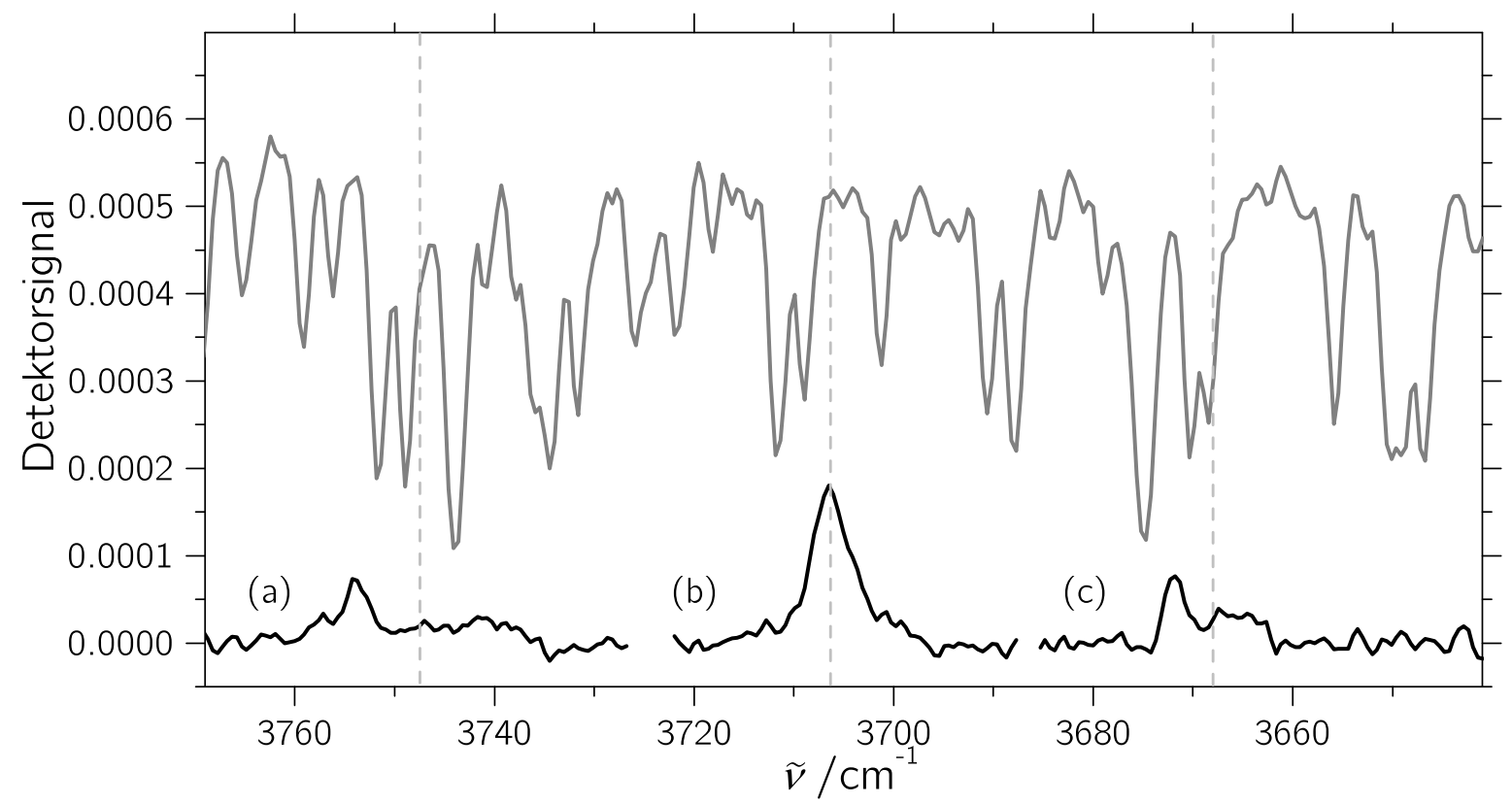

Abbildung 3.10: Gegenüberstellung der Wasserabsorptionsbanden (oben) mit drei verschiedenen OPO-Spektren. Spektren (a) und (c) zeigen stark asymmetrische Bandenformen, da sie mit Absorptionsbanden von Wasser zusammenfallen, während (b) nahezu ein Gaußprofil besitzt. Die grau gestrichelten Linien geben die ungefähre Bandenmitte wieder. 


\subsubsection{Einkopplung des OPO-Lasers in die Vakuumkammer und Justierung}

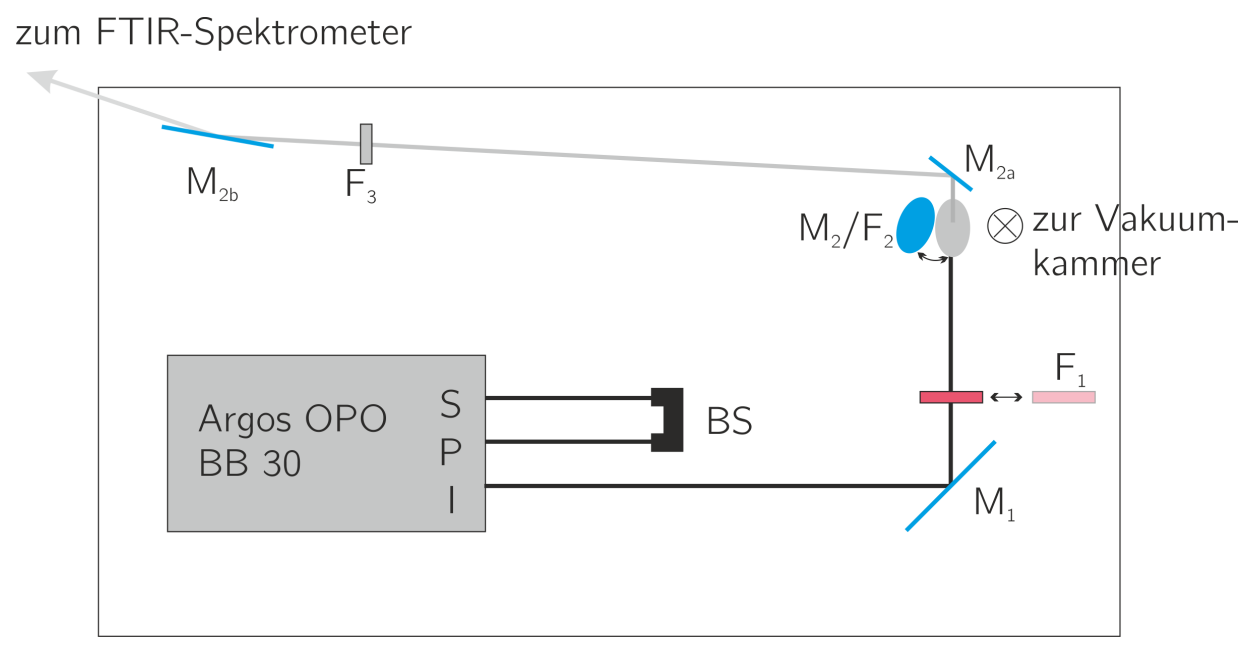

Abbildung 3.11: Schematischer Strahlengang des Idlers. Mit M werden Spiegel und mit F Filter bezeichnet. Signal- und Pumpstrahl werden durch einen Strahlfänger (BS) abgefangen. Mittels eines Klapphalters kann zwischen Einkopplung in die Kammer (Nutzung von $\mathrm{M}_{2}$ ) und in das FTIR-Spektrometer (Nutzung von $\mathrm{F}_{2}$ ) gewechselt werden.

Der Laser wurde auf einem Gestell, das an der Vakuumkammer angebracht ist, positioniert. Der Strahlengang ist in Abbildung 3.11 und 3.12 dargestellt. Mit einem Spiegel $\left(\mathrm{M}_{1}\right)$ wird der IR-Strahl um $90^{\circ}$ umgelenkt. Anschließend passiert er einen Filter $\left(\mathrm{F}_{1}\right)$, der das sichtbare Licht des Lasers entfernt (s. Kap. 3.3.3). Der Strahl wird danach entweder mittels eines Filters $\left(\mathrm{F}_{2}\right)$ abgeschwächt (s. Kap. 3.3.4) und mithilfe zweier Spiegel $\left(\mathrm{M}_{2 a}\right.$ und $\left.\mathrm{M}_{2 b}\right)$ und durch einen weiteren Filter $\left(\mathrm{F}_{3}\right)$ in Richtung des FTIRSpektrometers gelenkt oder er wird mittels eines Spiegels $\left(\mathrm{M}_{2}\right)$ senkrecht nach unten durch ein $\mathrm{CaF}_{2}$-Fenster, das im Brewsterwinkel montiert ist, in die Kammer gelenkt. Innerhalb der Kammer wird er dann mittels zweier weiterer Spiegel $\left(\mathrm{M}_{3}\right.$ und $\left.\mathrm{M}_{4}\right)$ direkt auf die Düse gelenkt, also antiparallel zur Expansionsrichtung eingekoppelt. Bei den verwendeten Spiegeln handelt es sich entweder um Silber-beschichtete Spiegel verschiedener Substrate oder um Hartgold-beschichtete Kupferspiegel.

Zur Fokussierung des Lasers auf den Raman-Anregungslaser wurden zwei verschiedene Ansätze erprobt. Der erste ist in Abbildung 3.12 dargestellt. Es wurde ein neues Düsendesign verwendet, bei dem die Schlitzdüse so modifiziert wurde, dass sich ein parabelförmiges Profil mit einer Brennweite von $3 \mathrm{~mm}$ ergibt. Eine technische Zeichnung der Düse ist im Anhang in Abbildung A.4.6 gezeigt. Trifft der IR-Laser auf dieses Profil, werden seine Randstrahlen reflektiert und als Linie im Abstand von $3 \mathrm{~mm}$ auf den Raman-Anregungslaser fokussiert. Hinter dem Fokus divergiert der Laserstrahl und 


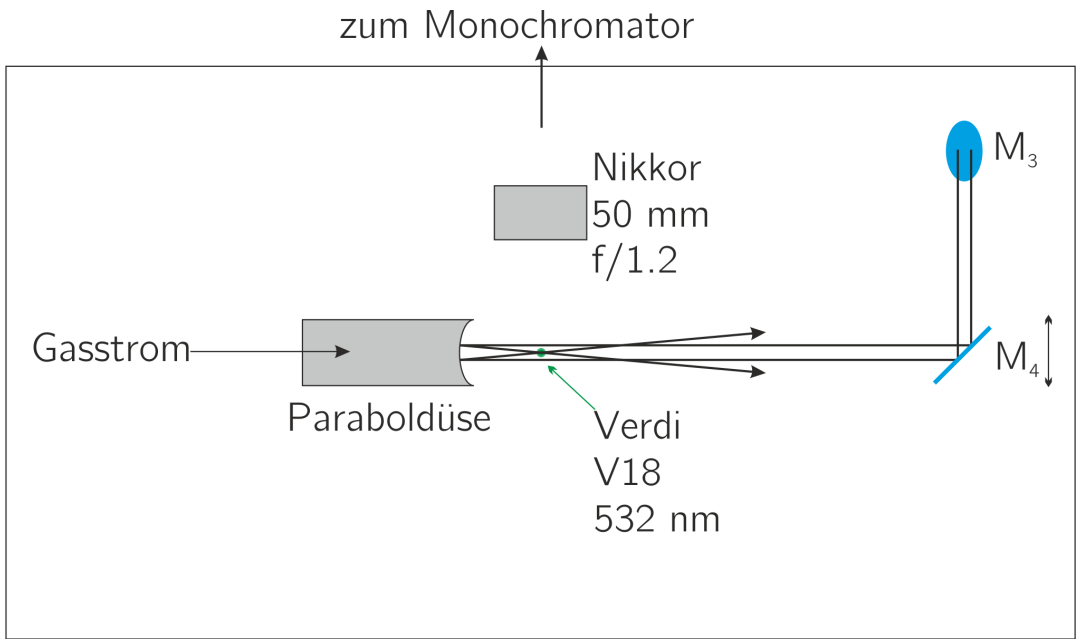

Abbildung 3.12: Strahlverlauf in der Vakuumkammer bei Nutzung der Paraboldüse.

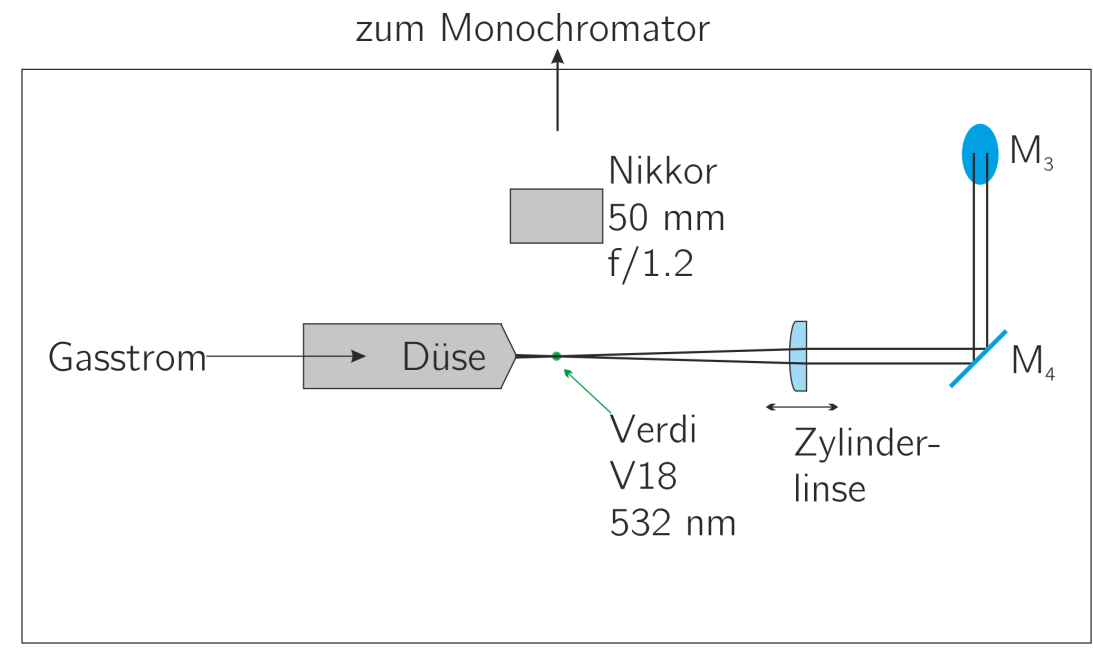

Abbildung 3.13: Strahlverlauf in der Vakuumkammer bei Nutzung der Zylinderlinse. Die Düse fungiert als Strahlfänger. 
trifft letztendlich auf die Wand der Vakuumkammer. Durch diese Anordnung kann auf einen Strahlfänger verzichtet werden.

Zur Justierung wird in folgender Weise vorgegangen. Bei evakuierter Kammer wird zunächst das Raman-Signal optimiert. Danach wird die Vakuumkammer belüftet und zunächst unter Nutzung des sichtbaren OPO-Lichts der Laserstrahl auf die Mitte der Paraboldüse gelenkt (Grobjustierung). Danach wird das sichtbare Licht mittels des Filters entfernt und mithilfe einer Fluoreszenzplatte der IR-Strahl verfolgt. Dieser Schritt ist nur notwendig, wenn das Si-Fenster verwendet wird, andernfalls kommt es zu keinem Strahlversatz und da sichtbares und IR-Licht überlappen, ist automatisch gewährleistet, dass die Justierung korrekt ist.

Danach muss allerdings der Leistungsverlust minimiert werden (Feinjustierung). Hierfür wird zunächst die Leistung am Laserkopf bestimmt. Anschließend wird das Leistungsmessgerät vor der Düse positioniert und die Leistung an dieser Stelle bestimmt. Durch Justierung der Spiegel M1-M4 sowie des $\mathrm{CaF}_{2}$-Fensters kann der Verlust auf ca. $15 \%$ minimiert werden. Im Folgenden wird die Kammer wieder evakuiert. Hiernach muss erneut das Kameraobjektiv justiert werden, die Düsenposition darf allerdings nicht weiter verändert werden. Die Bestimmung der Nullposition der Düse erfolgt wie in Ref. 9 beschrieben. Bei der Paraboldüse muss allerdings miteinbezogen werden, dass der Gasauslass um $0.7 \mathrm{~mm}$ weiter hinten liegt als die Spitze der Düse, mit der die Nullposition festgelegt wird. Der einzustellende Abstand zwischen Raman-Laser und Paraboldüse ist durch die Brennweite der Parabel auf $3 \mathrm{~mm}$ festgelegt.

Ein Nachteil des Ansatzes besteht darin, dass die Düse, die von der Werkstatt des IPC gefertigt wurde, wahrscheinlich nicht ideale Reflexionseigenschaften besitzt, so dass ein Teil der Strahlung beispielsweise durch eine raue Oberfläche zerstreut wird. Außerdem reflektiert die Düse den zentralen Teil der Strahlung nicht, so dass nicht die volle Leistung des Lasers genutzt werden kann.

Die zweite Möglichkeit ist in Abbildung 3.13 gezeigt. Der IR-Strahl wird mithilfe einer $\mathrm{CaF}_{2}$-Zylinderlinse (Korth Kristalle, $\mathrm{CaF}_{2}$-VUV-Zylinderlinse, plankonvex, $22 \mathrm{~mm} \times 22 \mathrm{~mm}, f(193 \mathrm{~nm})=55.82 \mathrm{~mm}$, optisch poliert) auf den Raman-Anregungslaser fokussiert. Es wird eine spitz zulaufende Schlitzdüse verwendet, die auch als Strahlfänger dient. Zur Justierung wurden verschiedene Ansätze erprobt. Als erste Möglichkeit wurde auch hier die sichtbare Emission des OPO genutzt. Die Düse wurde auf die Nullposition eingestellt und die Linse in der Kammer so eingestellt, dass der Fokus des roten Lichts genau auf der Düse liegt. Dann wurde die Wellenlänge von roter und infraroter Emission bestimmt. Mithilfe der Linsenformel kann hieraus die Differenz der Brennpunkte bestimmt werden. Die Linse wird so verschoben, dass nun der Fokus der infraroten Emission mit der Düsennullposition übereinstimmt. Danach kann die Düse auf den für die Messung gewünschten Abstand gebracht werden.

Eine weitere Idee bestand darin, die durch die rote Strahlung erzeugte Rayleighstreuung zu nutzen. Hierfür wurde zunächst ein Test mit dem Raman-Laser durchgeführt. 
Der Laser wurde auf eine Leistung von $20 \mathrm{~mW}$ eingestellt und bei evakuierter Kammer wurde das Verhältnis des Rayleigh-Signals ohne und mit Expansion bestimmt. Es zeigte sich, dass das Signal mit Expansion ungefähr dreimal so stark war wie ohne Expansion. Die ungefähre Leistung der roten Emission des OPO wurde mithilfe des SiFensters sowie durch Vergleich mit einem Justierlaser auf ungefähr $1-3 \mathrm{~mW}$ bestimmt. Die Ergebnisse des Raman-Lasers konnten jedoch nicht für den OPO-Laser reproduziert werden. Das liegt höchstwahrscheinlich an der Geometrie der Einkopplung. Die Methode funktioniert nur, wenn die Rayleighstreuung die Reflexion überwiegt. Da der OPO jedoch frontal auf die Düse trifft, sind die Reflexionen sehr viel stärker als die Rayleighstreuung, so dass auf diese Weise keine genaue Justierung möglich ist.

Als weitere Möglichkeit wurde genutzt, dass die Düse bei Justierung des Fokus direkt auf sie punktuell so stark erhitzt wurde, dass sie unter Vakuumbedingungen anfing zu leuchten. Die Stärke des Leuchtens hängt dabei besonders davon ab an welcher Stelle die Düse getroffen wird. Das bedeutet, wenn die Linse exakt justiert ist, trifft der Laser genau in den Düsenschlitz, wodurch die Emission minimal wird. Dieser Effekt zeigt sich, wenn die Linse vor- und zurückbewegt wird, durch einen Einbruch in der Emission.

Zur Justierung wurde folgendermaßen vorgegangen. Zunächst wurde das sichtbare OPO-Licht zur Vorjustierung bei belüfteter Kammer verwendet. Hierbei wurde auch besonders darauf geachtet, dass die OPO-Strahlung senkrecht auf die Düse trifft, so dass es beim Verstellen von Düse oder Linse keinen Versatz gibt. Nach Evakuieren der Kammer wurde dann zunächst eine Einstellung gewählt, bei der die Düse sehr stark leuchtet. Ausgehend hiervon wurde der Punkt der minimalen Emission durch Verfahren der Linse bestimmt. Das größte Problem an dieser Methode stellt die Bestimmung der Leuchtkraft dar. In Ermangelung eines geeigneten Messgeräts (wie z.B. einer Photodiode) wurde die Stärke der Emission im sichtbaren Spektralbereich zunächst nach Augenmaß bestimmt. Hierbei ist jedoch zu beachten, dass der Lichteindruck sehr stark von den äußeren Gegebenheiten abhängt und natürlich auch individuell verschieden ist. Die Reproduzierbarkeit wurde mithilfe mehrerer Personen geprüft. Es konnte festgestellt werden, dass der Erfolg der Methode stark davon abhängt wie genau die Vorjustierung ausgeführt wurde. Außerdem ist der Unterschied der Strahlungsleistung im sichtbaren Bereich beispielsweise bei einer Temperatur von $1020 \mathrm{~K}$ bereits 1.5 mal so groß wie für $1000 \mathrm{~K}^{\mathrm{a}}{ }^{\mathrm{a}}$ Ein Temperaturunterschied von lediglich $20 \mathrm{~K}$ hat also einen sehr großen Einfluss auf die wahrnehmbare Leuchtkraft. Bereits bei einer geringen Leistungszunahme (ca. 0.4 W) kann das Leuchten so stark werden, dass es nach Minimierung immer noch zu stark ist um Raman-Spektren mit akzeptablen S/N-Verhältnis aufzunehmen. Bei Anwendung der Methode empfiehlt es sich somit die Leistung des OPO-Lasers auf $5 \mathrm{~W}$ zu begrenzen. Ein weiteres Problem besteht darin, dass die Methode nicht auch für die Voroptimierung bei belüfteter Kammer genutzt werden kann, da durch die Wärmeabfuhr an Luft keine Emission wahrzunehmen ist. Durch den Einsatz einer

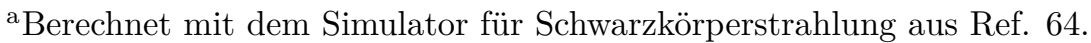


Photodiode könnte die Methode verbessert werden. Außerdem soll durch ein anderes Herstellungsverfahren die Düsengeometrie verbessert werden. In Abbildung A.4.7 im Anhang sind die Profile der alten Schlitzdüse und der neuen, durch Drahterosion hergestellten Schlitzdüse gezeigt. Die alte Schlitzdüse besitzt ein eher elliptisches Profil, während die neue ein rechteckiges Profil besitzt. Hierdurch könnte sich der Erfolg der Justiermethode ebenfalls verbessern, da die Breite des Düsenschlitzes bei dem neuen Herstellungsverfahren über die gesamte Länge des Düsenschlitzes gleich ist und daher die exakte Justierung der Düsenhöhe nicht kritisch ist.

Ein weiteres Problem der Anordnung besteht darin, dass die verwendete Zylinderlinse keine Antireflexbeschichtung besitzt, so dass ein Teil der Strahlung durch Reflexion verloren geht. Erste Ergebnisse von Testmessungen mit Methanol sowie eine ausführliche Diskussion weiterer Probleme sind in Kapitel 7 dargestellt.

\subsection{Spektrenauswertung und -abbildung}

\section{Bestimmung von Intensitäten}

\section{Integration}

Zur Bestimmung der Linien- oder Bandenintensitäten, die für die Temperaturbestimmung notwendig sind, wurde in frühen Auswertungen mit der zum verwendeten und den sonstigen in der Arbeitsgruppe vorhandenen FTIR-Spektrometern gehörigen Software OPUS (Bruker Optik GmbH, Ettlingen, Deutschland, Versionen 6.0, 6.5 und 7.0) gearbeitet. Dieses Programmpaket ist aber auf äquidistante Datenpunkte in der Wellenzahlskala angewiesen und rechnet beim Import von Raman-Spektren, deren Datenpunkte naturgemäß nicht äquidistant in der Wellenzahlskala sind, auf eine äquidistante Wellenzahlskala um. Dementsprechend ist es ungeeignet zur Auswertung von RamanSpektren. Da dieser Befund erst zu einem späteren Zeitpunkt festgestellt wurde, kann es zu Abweichungen von bis zu $9 \mathrm{~K}$ der in dieser Arbeit dargestellten Werte gegenüber schon publizierten Werten kommen (Ref. 15, ESI). Alle hier gezeigten Integrationswerte wurden mit dem Quickintegrations-Werkzeug des Programms Origin (OriginPro 8.5, OriginLab, Northampton, MA) bestimmt, wobei als Basislinie eine gerade Linie verwendet wurde.

\section{Fitfunktionen}

In einigen Fällen wurden auch Lorentzfunktionen an die gemessenen Spektren angepasst. Hierfür wurde das Quickfittool des Programms Origin (OriginPro 8.5, OriginLab, Northampton, MA) verwendet. 


\section{Hintergrundspektren und gemittelte Spektren}

Besonders für die mit dem MC205f-Monochromator aufgenommenen Spektren mussten aufgrund der gekrümmten Basislinie Hintergrundspektren aufgenommen werden. Hierzu wurde eine Expansion des jeweiligen Trägergases bei gleichbleibenden Messbedingungen aufgenommen. Diese wurden mithilfe von Origin (OriginPro 8.5, OriginLab, Northampton, MA) von den Substanzspektren subtrahiert. In einigen Fällen konnten Spektren, die an verschiedenen Messtagen aber zu gleichen Bedingungen (auch hinsichtlich der Qualität der Justierung) aufgenommen wurden, gemittelt werden. Auch hierfür wurde mit Origin (OriginPro 8.5, OriginLab, Northampton, MA) gearbeitet.

\section{IR-Spektren}

IR-Spektren wurden weiterhin mit OPUS (Version 7.0) ausgewertet, da hier das oben genannte Problem nicht auftaucht.

\section{Abbildungen}

In dieser Arbeit gezeigte Abbildungen wurden entweder mit Origin (OriginPro 8.5, OriginLab, Northampton, MA), mit CorelDRAW ${ }^{\circledR}$ X5 (Corel Corporation, Ottawa, CA) oder mit InkScape (Version 0.48.4) erstellt. 


\section{Temperaturbestimmung in der Jetexpansion}

Die lokale Temperatur dient als wichtiges Charakteristikum der Überschallexpansionen. Da hier aber eine Nicht-Gleichgewichtssituation vorliegt, in der somit keine Boltzmannverteilung existiert, ist eine generelle Definition der Temperatur kaum möglich. Innerhalb der verschiedenen Freiheitsgrade liegen jedoch näherungsweise lokale Boltzmannverteilungen vor, was die Definition von Translations-, Rotations- und Schwingungstemperaturen ermöglicht. ${ }^{65}$

Zur Bestimmung von Rotationstemperaturen zweiatomiger und linearer mehratomiger Moleküle kann die relative Population der Rotationsniveaus wie in Kapitel 2.3 vorgestellt bestimmt werden. ${ }^{8,9,65}$ Bei nicht spektral aufgelösten Rotationsübergängen wird auch der Abstand der P- und R-Zweig-Maxima genutzt. ${ }^{57}$ Für komplexere Moleküle werden daher Spektrendatenbanken oder Simulationsprogramme zu Hilfe genommen. ${ }^{22,57}$

Zur Bestimmung von Schwingungstemperaturen dienen relative Populationen von Fundamentalen und heißen Banden, ${ }^{8,65-67}$ wobei hier zur Abschätzung zumindest die relativen Übergangsmomente, die beispielsweise aus einer Referenzmessung bei bekannter Temperatur bestimmt werden können, bekannt sein müssen. ${ }^{8}$

Generell ist für die Temperaturbestimmung in der Überschallexpansion zu beachten, dass unterschiedliche im Jet vorhandene Spezies getrennt voneinander betrachtet werden sollten, da sie sich unterschiedlich verhalten können. Das schließt auch unterschiedliche Kernspinisomere eines Monomers ${ }^{68}$ ein.

Die Temperaturbestimmung der Rotationsfreiheitsgrade unter Nutzung der relativen Intensitäten der verschiedenen Übergänge im Stokes-Bereich, die u.a. für zweiatomige Moleküle verwendet werden kann, (im weiteren als "Auftragung gegen $J(J+1)$ “ bezeichnet) wurde bereits von P. Zielke und T. Wassermann zur Charakterisierung der Jetexpansion im Curry-Jet erprobt. ${ }^{8,9}$ Diese etablierte Methode soll zur Evaluierung der in Kapitel 2.3 vorgestellten Stokes-Anti-Stokes-Methode (S/AS), die in dieser Arbeit zur Bestimmung der Temperaturen im Jet angewendet wurde, dienen.

Das Stokes-Anti-Stokes-Intensitätsverhältnis wurde bereits zur Bestimmung von lokalen Heizeffekten an Polymeren ${ }^{69}$ und zur Temperaturbestimmung beispielsweise von 
Flammen ${ }^{70}$ und Mikropartikeln ${ }^{71}$ oder in Wasserproben ${ }^{72}$ sowie in OLEDs ${ }^{73}$ angewendet. Für die Überschallexpansion existieren bislang nur wenige Publikationen, in denen die Methode genutzt wird. ${ }^{14,15}$

Die Vorteile des Verfahrens liegen in der einfachen Anwendbarkeit, so müssen weder Streuquerschnitte noch die Zuordnung der Linien oder Banden zu bestimmten Übergängen bekannt sein, so dass eine rein empirische Abschätzung der Temperatur möglich ist. Allerdings ist die Methode in der Überschallexpansion aufgrund der tiefen Temperaturen nur auf niederfrequente Banden anwendbar. ${ }^{9}$ In Kapitel 4.1 wird das Auswertungsverfahren zunächst anhand eines einfachen Testsystems erprobt und danach auf komplexere Systeme angewendet.

\subsection{Rotationstemperaturen}

\subsubsection{Testsystem Stickstoff}

Als einfaches Testsystem dienen die Rotationsübergänge des Stickstoff-Moleküls, die aufgrund ihrer großen Raman-Aktivität gut geeignet sind. Für die Testmessungen wurde eine Mischung von 2\% Stickstoff in Helium verwendet. Zunächst wurden Gasphasenspektren bei Raumtemperatur von ca. $300 \mathrm{~K}$ aufgenommen. Um die Rayleighstreuung zu entfernen wurden zwei verschiedene Ansätze erprobt. Zum einen wurden die in Kapitel 3.1 beschriebenen Kantenfilter verwendet, zum anderen wurde ein Polfilter (Hama Hoya Polfilter linear 450/670, e67 mit Drehfassung) eingesetzt. Der Polfilter kann genutzt werden, da bei der Rayleighstreuung die Polarisationsrichtung weitgehend erhalten bleibt, während die Raman-Streuung der Rotationsübergänge vollständig depolarisiert (Depolarisationsverhältnis von 0.75) ist, so dass die Rayleighstreuung deutlich stärker abgeschwächt wird als die Raman-Streuung. Allerdings ist aufgrund des großen Anteils an Rayleighstreuung die Rayleighlinie trotz Filter immer noch sehr intensiv, so dass zum Schutz der CCD-Kamera nur sehr kurze Messzeiten von 1-2s verwendet wurden. Die Kantenfilter hingegen unterdrücken die Rayleighlinie mindestens um den Faktor $10^{6}$, schneiden jedoch auch diejenigen Rotationslinien, die besonders nah an der Rayleighlinie liegen ab, so dass besonders bei kalten Temperaturen nur noch sehr wenige Linien zur Auswertung genutzt werden können. Außerdem muss zwischen den beiden Filtern gewechselt werden um einmal ein Stokes- und einmal ein Anti-Stokes-Spektrum aufzunehmen. Da die Filter im fokussierten Strahl eingesetzt sind, kommt es hierdurch zu Veränderungen der Justierung, also zu Signaleinbußen und somit zu einer Verfälschung der Intensitäten. Eine Nachjustierung nach dem Filterwechsel ist besonders bei Jetexpansionen aufgrund der geringen Signalstärke der zur Justierung verwendeten Rotationslinien von Luft nicht möglich, außerdem birgt dies die Gefahr der Überkompensation. Aus diesem Grund wurde auf eine Nachjustierung verzichtet und vielmehr die Größe des Fehlers anhand von Gasphasenspektren bestimmt. Für den Filterwechsel 
wurde ein Filterrad genutzt, so dass ein Verkippen der Filter und hierdurch weitere Signalverschlechterungen ausgeschlossen werden können. Aufeinander folgende Messungen, bei denen alternierend Stokes- und Anti-Stokes-Spektren aufgenommen wurden, ergaben, dass der durch den Filterwechsel entstehende Fehler robust ist. So können beispielsweise auch Spektren, die nicht direkt hintereinander aufgenommen wurden, zur Auswertung genutzt werden, ohne dass sich Mittelwert und Fehlergröße deutlich ändern.

Trotz dieser Nachteile ist die Nutzung der Kantenfilter besonders für die in Kapitel 4.2 beschriebene Bestimmung von Schwingungstemperaturen unabdingbar, da hier erstens sehr viel längere Messzeiten als für die Rotationsspektren benötigt werden und zweitens durch Nutzung des Polfilters der Q-Zweig der Schwingung, in dem häufig der größte Teil der Intensität enthalten ist, stark abgeschwächt wird. Hierdurch wären besonders die Anti-Stokes-Spektren aufgrund ihrer deutlich geringeren Signalstärke nur noch bedingt auswertbar.

Neben den experimentellen Spektren wurden außerdem aus Gleichung 2.14 und 2.15 theoretische Spektren für Stickstoff in der Näherung des starren Rotators berechnet, die mit den gemessenen Spektren verglichen werden können. Für die Rotationskonstante $\widetilde{B}_{0}$ von Stickstoff wird hierbei ein Wert von $1.989574 \mathrm{~cm}^{-1}$ verwendet. ${ }^{74}$

In Abbildung 4.1 sind Gasphasenspektren für eine Temperatur von ca. $300 \mathrm{~K}$ gezeigt, und zwar das berechnete (a) und das mit dem Polfilter gemessene Spektrum (b) sowie die beiden Spektren (c), die mit dem Stokes- (c, schwarz) und Anti-Stokes-Kantenfilter (c, grau) aufgenommen wurden. In Spektrenpaar (c) ist der Bereich, in dem eine Auswertung der Intensitäten aufgrund der Filterkanten nicht möglich ist, durch graugestrichelten Linien gekennzeichnet.

Es zeigt sich, dass sowohl die relativen Intensitäten von Stokes- und Anti-Stokes-Linien als auch der Intensitätsverlauf der verschiedenen Rotationsübergänge innerhalb des Stokes- bzw. Anti-Stokes Bereichs für Spektren (a) und (b) sehr gut übereinstimmen. Die relative Intensität der Spektren (b) und (c) entspricht den Erwartungen für ein Depolarisationsverhältnis der Rotationslinien von 0.75 unter Berücksichtigung, dass der Monochromator in diesem Wellenlängenbereich für senkrecht polarisiertes Licht ca. 1.5 mal effizienter ${ }^{11}$ ist. Bei Spektrenpaar (c) fällt außerdem auf, dass die Intensität der Anti-Stokes-Linien relativ zu den Stokes-Linien insgesamt deutlich geringer als in den anderen Spektren ist. Des Weiteren ist die Intensität der ersten Anti-Stokes-Linie nach der Filterkante deutlich abgeschwächt, so dass davon ausgegangen werden muss, dass hier eine Verfälschung durch den Filter vorliegt. Daher wurde die Auswertung grundsätzlich erst ab der zweiten Rotationslinie nach der Filterkante durchgeführt. Beim Vergleich der Intensitätsmaxima der Rayleighlinien fällt auf, dass diese bei Nutzung des Stokes-Kantenfilters bereits bei einer Messzeit von $5 \mathrm{~s}$ übersättigt (>65000 counts) ist, während bei Nutzung des Anti-Stokes-Filters lediglich 670 counts erreicht werden, was sowohl auf die bessere Unterdrückung der Rayleighstreuung (laut Hersteller >OD 


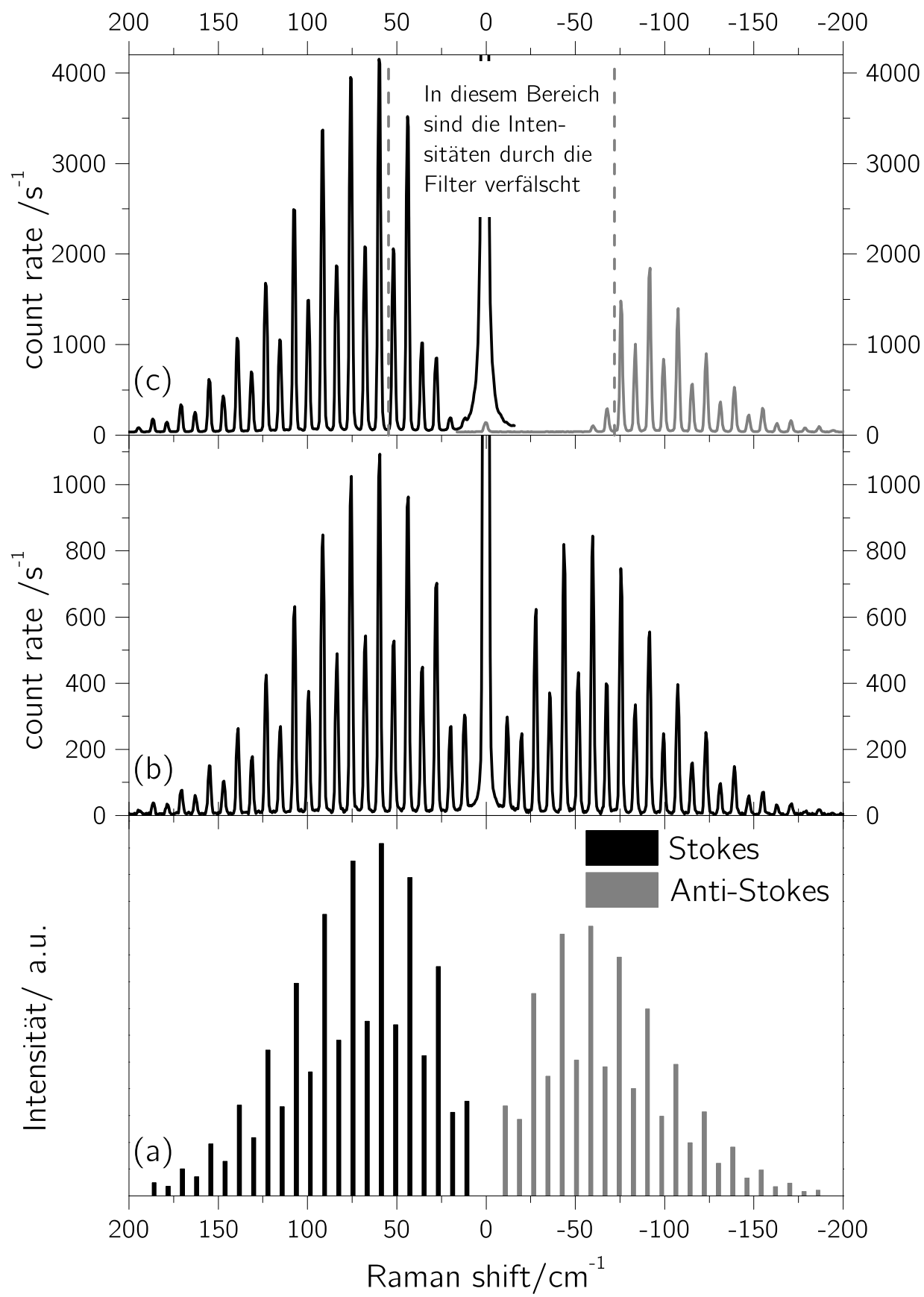

Abbildung 4.1: Vergleich von theoretischem und experimentellen RamanRotationsspektren von Stickstoff in der Gasphase bei ca. 300 K. (a) Berechnetes Raman-Rotationsspektrum von Stickstoff bei $300 \mathrm{~K}$, (b) mithilfe eines Polfilters aufgenommenes experimentelles Spektrum von 2\% Stickstoff in Helium und (c) schwarz: experimentelles Stokes-Spektrum und grau: experimentelles Anti-Stokes-Spektrum von jeweils $2 \%$ Stickstoff in Helium jeweils bei $293 \mathrm{~K}$. Die grau-gestrichelte Linie gibt die Wellenzahl an, ab der jeweils die Filterkante beginnt. (Exp.-Bed.: s. Anhang A.3.) 


\begin{tabular}{lll}
\hline Gasphase (60 mbar) & Methode & $\bar{T}_{\text {rot }} / \mathrm{K}$ \\
\hline Polfilter & $J(J+1)$ & $291 \pm 3$ \\
& S/AS & $300 \pm 30$ \\
Kantenfilter & $J(J+1)$ & $299 \pm 8$ \\
& S/AS & $250 \pm 40$ \\
& S/AS korr & $270 \pm 40$ \\
Labortemperatur: & & 293 \\
\hline
\end{tabular}

Tabelle 4.1: Vergleich der Mittelwerte der Rotationstemperatur von Stickstoff für Gasphasen-Spektren (60 mbar). Jeweils ermittelt aus Spektren, die mit Pol- oder Kantenfiltern gemessen wurden und jeweils aus einer Auftragung gegen $J(J+1)$ und aus dem Stokes/Anti-Stokes Intensitätsverhältnis. Für die Messungen bei Nutzung der Kantenfilter wurden außerdem noch korrigierte Werte ermittelt, die sowohl die polarisationsrichtungsspezifische Effizienz des Monochromators als auch die unterschiedlichen Transmissionseigenschaften der Kantenfilter miteinbeziehen.

6 anstelle von OD 6 für den Stokes-Filter) als auch auf die generellen Signaleinbußen durch den Filterwechsel zurückzuführen ist. Für den Polfilter wurde bei einer Messzeit von $1 \mathrm{~s}$ ein Wert von 24000 counts erreicht. Hier ist eine Erhöhung der Messzeit um ein besseres Signal-zu-Rausch-Verhältnis zu erhalten noch möglich.

Die Intensität der gemessenen Rotationslinien wurde durch Integration bestimmt. Die Wellenzahlen der Übergänge wurden in einem der gemessenen Spektren bestimmt und für die übrigen Spektren übernommen. Die Kalibrierung erfolgte abweichend von der üblichen Methode (Ne-Linien) über die Literatur-bekannten Übergänge von Stickstoff, da hierdurch robustere Werte erzielt werden konnten. Zur Temperaturbestimmung wurden bei Spektrum (b) die ersten 20 Rotationslinien verwendet, bei Spektrenpaar (c) die Rotationsübergänge 9-26. Die Zuordnung erfolgte gemäß Ref. 74. Die Bestimmung der Temperatur erfolgte gemäß den Gleichungen 2.13 und 2.17. Die Ergebnisse sind in Tabelle 4.1 aufgeführt, wobei hier die Mittelwerte der über die zwei verschiedenen Methoden bestimmten Temperaturen sowie deren Standardabweichungen angegeben sind. Vollständige Tabellen für Spektrum (b) und Spektrenpaar (c) mit allen Wertepaaren der Stokes-Anti-Stokes Auswertung und den jeweils vier Werten aus der Auftragung sowie exemplarisch die Auftragung gegen $J(J+1)$ für Spektrum (b) sind im Anhang in Tabellen A.5.1-A.5.3 bzw. in Abbildung A.5.1 aufgeführt. Als Plausibilitätsprüfung wurde auch das berechnete Spektrum ausgewertet. Hier ergeben sich bei Auswertung nach Stokes-Anti-Stokes-Verhältnis für alle Wellenzahlen erwartungsgemäß Temperaturen von $300 \mathrm{~K}$ und bei der Auftragung gegen $J(J+1)$ Werte von $298-301 \mathrm{~K}{ }^{\text {a }}$

Die in Tabelle 4.1 aufgeführten Werte zeigen, dass die Fehlerbalken bei der Auftragung gegen $J(J+1)$ deutlich kleiner als für die Stokes-Anti-Stokes Methode sind. Bei letzterer

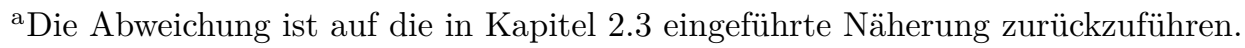


können sich besonders für die ersten beiden Rotationsübergänge Abweichungen von der gemessenen Temperatur von mehr als $50 \mathrm{~K}$ ergeben. Dies ist auf den bei Raumtemperatur für diese Linien vorliegenden geringen Besetzungsunterschied zurückzuführen, der dafür sorgt, dass die Methode sehr empfindlich auf geringe Veränderungen in der Bestimmung des Integrals reagiert. Anhand einer Fehlerfortpflanzung von Gleichung 2.13 lässt sich das gut erkennen. Der Fehler der Temperatur ist laut Fehlerfortpflanzung (Größtfehler) wie folgt vom Intensitätsverhältnis $I_{\mathrm{S} / \mathrm{AS}}$ abhängig:

$$
\begin{aligned}
\Delta T & =\left|\frac{\partial T}{\partial I_{\mathrm{S} / \mathrm{AS}}}\right| \cdot \Delta I_{\mathrm{S} / \mathrm{AS}} \\
& =\frac{h c_{0} \widetilde{\nu}_{\mathrm{k}}}{k \cdot I_{\mathrm{S} / \mathrm{AS}}\left(\ln I_{\mathrm{S} / \mathrm{AS}}-\ln \frac{\left(\widetilde{\nu}_{0}-\widetilde{\nu}_{\mathrm{k}}\right)^{3}}{\left(\widetilde{\nu}_{0}+\widetilde{\nu}_{\mathrm{k}}\right)^{3}}\right)^{2}} \cdot \Delta I_{\mathrm{S} / \mathrm{AS}} \\
& =T \cdot \frac{\Delta I_{\mathrm{S} / \mathrm{AS}}}{I_{\mathrm{S} / \mathrm{AS}} \cdot\left(\ln I_{\mathrm{S} / \mathrm{AS}}-\ln \frac{\left(\widetilde{\nu}_{0}-\widetilde{\nu}_{\mathrm{k}}\right)^{3}}{\left(\widetilde{\nu}_{0}+\widetilde{\nu}_{\mathrm{k}}\right)^{3}}\right)}
\end{aligned}
$$

Als relativer Fehler ergibt sich:

$$
\frac{\Delta T}{T}=\frac{\Delta I_{\mathrm{S} / \mathrm{AS}}}{I_{\mathrm{S} / \mathrm{AS}} \cdot\left(\ln I_{\mathrm{S} / \mathrm{AS}}-\ln \frac{\left(\widetilde{\nu}_{0}-\widetilde{\nu}_{\mathrm{k}}\right)^{3}}{\left(\widetilde{\nu}_{0}+\widetilde{\nu}_{\mathrm{k}}\right)^{3}}\right)}
$$

Bei Gleichbesetzung der Stokes- und Anti-Stokes Niveaus (Grenzfall $I_{\mathrm{S} / \mathrm{AS}} \rightarrow 1$ ) ergibt sich:

$$
\frac{\Delta T}{T}=\frac{\Delta I_{\mathrm{S} / \mathrm{AS}}}{-\ln \frac{\left(\widetilde{\nu}_{0}-\widetilde{\nu}_{\mathrm{k}}\right)^{3}}{\left(\widetilde{\nu}_{0}+\widetilde{\nu}_{\mathrm{k}}\right)^{3}}}
$$

Der relative Fehler also ist direkt proportional zum Fehler des Intensitätsverhältnisses und außerdem umso größer je kleiner die Wellenzahl des Übergangs ist. Die optimalen Wellenzahlen, die zur Temperaturbestimmung genutzt werden sollten, liegen laut Ref. $75 \mathrm{im}$ Bereich $k T<h c \widetilde{\nu}<4 k T$, also für $300 \mathrm{~K}$ zwischen $210 \mathrm{~cm}^{-1}$ und $830 \mathrm{~cm}^{-1}$.

Beim Vergleich der Ergebnisse aus Spektrum (b) und (c) fällt auf, dass die Temperaturen aus der S/AS-Methode für Spektren (c) deutlich zu niedrig bestimmt werden. Ein Teil dieses Fehlers ist auf die schlechteren Transmissionseigenschaften des Anti-StokesFilters und auf die wellenlängenspezifische Effizienz des Monochromators für parallel und senkrecht polarisiertes Licht zurückzuführen. Es wurde ein Korrekturfaktor von $5 \%$ der Linienintensität abgeschätzt (3\% Monochromatorfehler $+2 \%$ Filterfehler), mit dem die Anti-Stokes-Integrale korrigiert wurden. Die korrigierten Werte $\left(\mathrm{S} / \mathrm{AS}_{\text {korr }}\right)$ sind jedoch immer noch systematisch zu niedrig. Dementsprechend können als größte Fehlerquelle die Signaleinbußen, die durch den Filterwechsel entstehen, angesehen werden. 


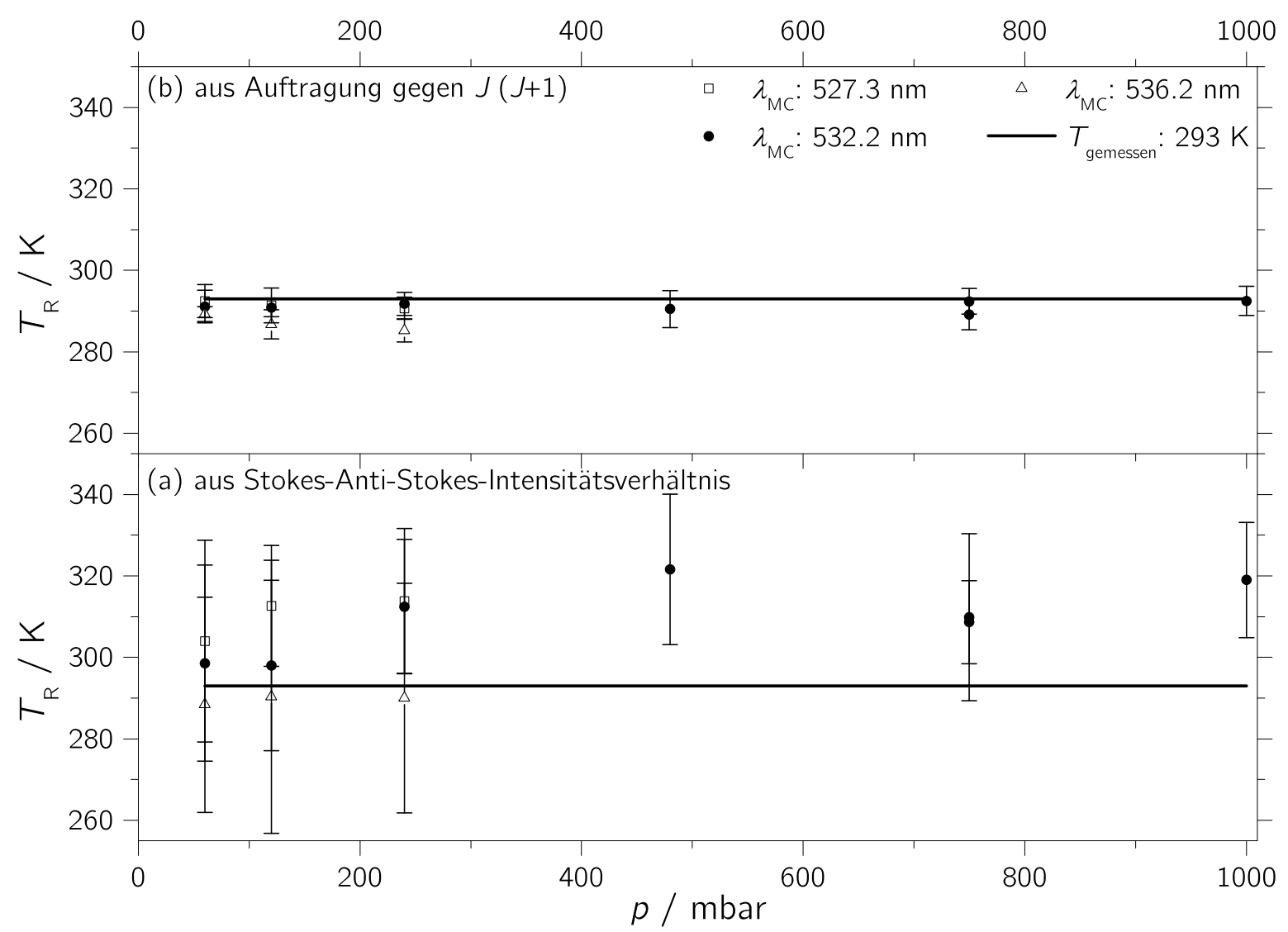

Abbildung 4.2: Druck- und Pixelbereich-Abhängigkeit der ermittelten Rotationstemperaturen. (a) aus dem S/AS-Intensitätsverhältnis bestimmte mittlere Rotationstemperaturen inkl. Standardabweichungen. (b) aus der Auftragung gegen $J(J+1)$ ermittelte Werte. Für 60-240 mbar wurde geprüft, ob die CCD-Kamera unterschiedliche Pixelsensitivitäten besitzt, indem verschiedene Wellenlängen am Monochromator $\left(\lambda_{\mathrm{MC}}\right)$ eingestellt wurden.

Da durch die Messmethode mit dem Polfilter Stokes- und Anti-Stokes-Bereich gleichzeitig aufgenommen werden können, wurde diese Methode verwendet um weitere Aspekte des Experiments zu prüfen. Zum einen wurde die Robustheit der Ergebnisse der Gasphasenmessungen in Abhängigkeit vom Druck geprüft. Zum anderen wurde evaluiert, ob der CCD-Chip verschiedene Pixelsensitivitäten besitzt. Hierfür wurden Spektren bei unterschiedlichen Monochromatorwellenlängen aufgenommen, so dass die Rotationslinien im linken, rechten oder mittleren Bereich des CCD-Chips lagen. Die Ergebnisse der beiden Messreihen sind in Abbildung 4.2 gezeigt. Es wurden die Mittelwerte aller Rotationslinien bzw. aller Auftragungen sowie deren Standardabweichung als Fehlerbalken verwendet. Es zeigt sich erneut, dass die $J(J+1)$-Auftragung deutlich näher am gemessenen Temperaturwert liegt und auch die Fehlergrenzen deutlich geringer sind. Bei der Stokes-Anti-Stokes Methode kommt es bei den Mittelwerten zu Abweichungen um bis 
zu $20 \mathrm{~K}$, was auf die oben erwähnte Empfindlichkeit der Methode auf die Integration zurückzuführen ist. Außerdem zeigt sich, dass der CCD-Chip leicht unterschiedliche Pixelsensitivitäten aufweist. So wird die Temperatur bei geringer Monochromatorwellenlänge (entsprechend dem Pixelbereich 500-1340) systematisch größer bestimmt als bei großer Monochromatorwellenlänge (entsprechend dem Pixelbereich 0-700). Allerdings sind die Unterschiede im Rahmen der Fehlergrenzen klein.

Insgesamt erscheint die Stokes/Anti-Stokes-Methode mit Abweichungen von maximal $16 \%$ (unkorrigierte Werte bei Nutzung der Kantenfilter) vom per Thermometer bestimmten Wert als gut geeignet.

Beim Übergang zur Jetexpansion zeigt sich, dass die Rotationslinien maximal bis zu einer Wellenzahl von $100 \mathrm{~cm}^{-1}$ sinnvoll auswertbar sind, da die Besetzung für höhere Werte zu gering ist. Abbildung 4.3 ist analog zu Abbildung 4.1 aufgebaut. Spektrum (a) ist ein für eine Temperatur von $40 \mathrm{~K}$ berechnetes Spektrum; Spektren (b) und (c) sind am Curry-Jet bei einem Abstand von $1 \mathrm{~mm}$ zur Düse aufgenommene Spektren, wobei für (b) der Polfilter und für (c) die Kantenfilter verwendet wurden. Bereits durch Vergleich von Spektrum (b) mit berechneten Spektren bei verschiedenen Temperaturen konnte die Rotationstemperatur auf $30-50 \mathrm{~K}$ abgeschätzt werden. Die Übereinstimmung zwischen Spektrum (a) und (b) ist sehr gut während bei Spektrum (c) bedingt durch die Filter Abweichungen auftreten. Auch hier kann die Peakhöhe der Rayleighlinien verglichen werden. Da keines der Spektren übersättigt war können direkt die Zählraten genutzt werden. Für Spektrum (b) ergibt sich ein Wert von 32500 counts/s; für Spektrenpaar (c) Werte von 590 counts/s (Stokes) und 5 counts/s (Anti-Stokes). In Spektrum (b) ist die Rayleighlinie bei einer Messzeit von $2 \mathrm{~s}$ schon nahe an der Sättigungsgrenze, daher wurde auf eine weitere Erhöhung der Messzeit verzichtet, allerdings wäre durchaus noch eine Steigerung der Messzeit auf bspw. 3-4s möglich ohne den Detektor zu beschädigen.

Bei Spektrenpaar (c) ist durch den Bereich der Filterkanten die Anzahl an auswertbaren Linien sehr gering. Bei den in Tabelle 4.2 aufgeführten Werten handelt es sich um Mittelwerte, vollständige Tabellen aller auswertbaren Linien befinden sich im Anhang in Tabelle A.5.4 und A.5.5. Die Werte ergeben für die Auswertung nach $J(J+1)$ ein konsistentes Bild mit einer Temperatur von ca. $40 \mathrm{~K}$, während für die S/AS-Methode nur bei Spektrum (b) eine ähnliche Temperatur bestimmt werden konnte. Bei Spektrum (c) ergeben sich Temperaturwerte, die um $85 \%$ höher liegen als alle anderen Werte. Die Korrektur um 5\% führt zu einer weiteren Erhöhung der Werte. Die starke Abweichung ist einerseits auf die extrem geringen Intensitäten zurückzuführen andererseits auf den Filterwechsel. Die Messung wurde nur einmal durchgeführt, so dass hier auch möglicherweise eine Signalerhöhung, die zufällig durch den Filterwechsel auftrat erfolgt sein könnte. Allerdings müsste sich hierfür das Signal mehr als verdoppelt haben, was eher unwahrscheinlich ist. Hier stößt die Messmethode unter Nutzung der Kantenfilter 
an ihre Grenzen. Das Verfahren ist eher für Schwingungstemperaturen geeignet (vgl. Kap.4.2).

Unter Nutzung des Polfilters scheint die S/AS-Methode aber robust zu sein, daher wurde als weitere Charakterisierung die Rotationstemperatur im Jet in Abhängigkeit vom Düsenabstand bestimmt. Die Ergebnisse der beiden Auswertungsmethoden sind in Abbildung 4.4 dargestellt.

\begin{tabular}{lll}
\hline Jet $(d=1 \mathrm{~mm})$ & Methode & $\bar{T}_{\text {rot }} / \mathrm{K}$ \\
\hline Polfilter & $J(J+1)$ & $39 \pm 1$ \\
& S/AS & $40 \pm 4$ \\
Kantenfilter & $J(J+1)$ & $41 \pm 6$ \\
& S/AS & $74 \pm 9$ \\
& S/AS & $76 \pm 9$ \\
\hline
\end{tabular}

Tabelle 4.2: Vergleich der Mittelwerte der Rotationstemperatur von Stickstoff für Jetspektren bei $1 \mathrm{~mm}$ Abstand zur Düse. Jeweils ermittelt aus Spektren, die mit Pol- oder Kantenfiltern gemessen wurden und jeweils aus einer Auftragung gegen $J(J+1)$ und aus dem Stokes/Anti-Stokes Intensitätsverhältnis. Für die mit Kantenfilter durchgeführten Messungen wurden außerdem noch korrigierte Werte (S/AS korr) ermittelt, die sowohl die polarisationsrichtungsspezifische Effizienz des Monochromators als auch die unterschiedlichen Transmissionseigenschaften der Kantenfilter näherungsweise miteinbeziehen.

Auftragung (a) zeigt den Temperaturverlauf für die ersten fünf Rotationsübergänge sowie den Mittelwert dieser (rot). Außerdem ist noch der Mittelwert aller auswertbaren Übergänge angegeben (rot, gestrichelt). Die jeweilige Anzahl an auswertbaren Übergängen ist in Tabelle A.5.6 im Anhang zusammen mit den zugehörigen Temperaturen angegeben, sie beläuft sich auf 4-11. Die Übergänge sind mit der Ausgangs- und Endquantenzahl des Stokes-Rotationsübergangs bezeichnet. Der Übergang $(6 \leftarrow 4)$ konnte für den Abstand von $4 \mathrm{~mm}$ nicht mehr ausgewertet werden, daher fehlt hier ein Datenpunkt. Für den angegebenen Mittelwert wurden hier entsprechend die ersten vier Übergänge verwendet.

Bei Einbeziehung aller auswertbaren Übergänge ergeben sich ab einem Abstand von $6 \mathrm{~mm}$ Temperaturen, die im Mittel deutlich höher liegen als die der ersten fünf Rotationsübergänge. Diese Abweichung deutet darauf hin, dass die Annahme einer lokalen Boltzmannverteilung in diesem Bereich der Expansion ungültig ist. Die für die einzelnen Rotationsübergänge bestimmten Temperaturen steigen systematisch mit steigender Quantenzahl an, was auf eine überproportional große Besetzung dieser Zustände im Vergleich zu den niedriger gelegenen hinweist. Da in diesem Bereich jedoch nicht mehr die Ruhezone der Expansion geprobt wird, kann der Befund auch lediglich die Heterogenität der Expansion widerspiegeln. Hinweise hierauf zeigen sich auch in den Auftragungen gegen $J(J+1)$. Exemplarisch ist hierfür die Auftragung für einen Abstand von $8 \mathrm{~mm}$ zur Düse in Abbildung A.5.2 im Anhang aufgeführt. Es zeigt sich 


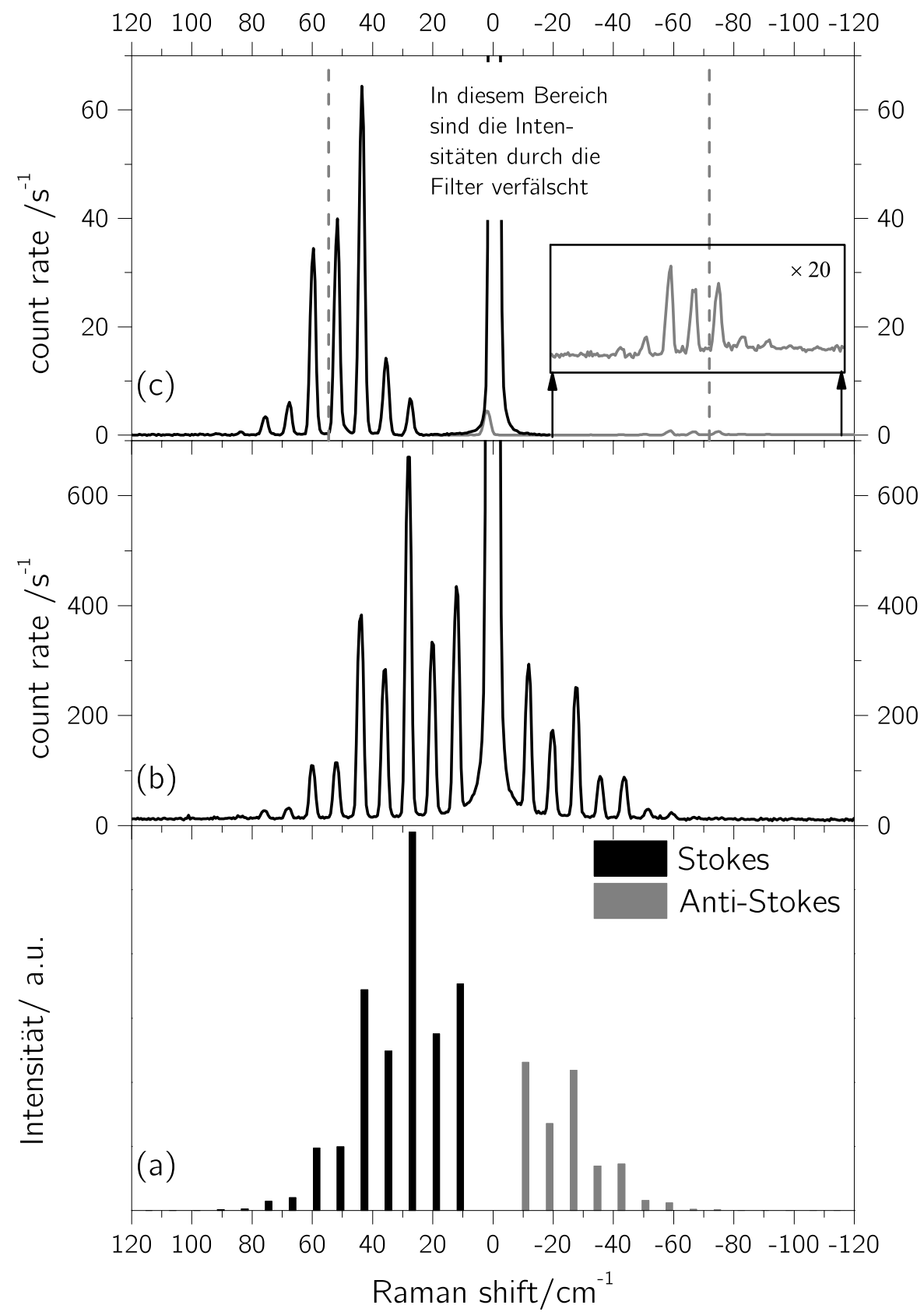

Abbildung 4.3: Vergleich von theoretischem und experimentellen RamanRotationsspektren von Stickstoff für die Jetexpansion. (a) berechnetes Spektrum bei einer Temperatur von $40 \mathrm{~K}$ (b) mit Polfilter aufgenommenes experimentelles Spektrum, (c) mit Stokes- (schwarz) und Anti-Stokes- (grau) Filter aufgenommene Spektren jeweils bei $1 \mathrm{~mm}$ Abstand zur Düse. Außerdem ist ein mit Faktor 20 skalierter Ausschnitt des Anti-Stokes-Spektrums (c, Kasten) gezeigt. Die Expansionsbedingungen sind in Anhang A.3 aufgeführt. Die grau-gestrichelte Linie gibt die Wellenzahl an, ab der jeweils die Filterkante beginnt. 
ein „Knick“ in der Auftragung der besonders für die geraden $J$ gut erkennbar ist. Für Rotationsquantenzahlen von $J \geq 8$ ergibt sich eine flachere Steigung und somit eine höhere Temperatur.

Auftragung (b) in Abbildung 4.4 zeigt den Temperaturverlauf, der sich aus der $J(J+1)$ Auftragung ergibt für Stokes- und Anti-Stokes-Übergänge und jeweils für gerade und ungerade Übergänge sowie den Mittelwert (rot). Auftragung (a) und (b) stimmen für die Mittelwerte aller auswertbaren Linien (a) und den Mittelwert von (b) gut überein. Auch hier ist allerdings zu erkennen, dass die Streuung um den Mittelwert für die Auftragung geringer ist als für die S/AS-Methode. Des Weiteren zeigt sich, dass die Temperaturen von geraden und ungeraden Übergängen sich nicht stärker unterscheiden als diejenigen unterschiedlicher Übergänge gleicher Parität. Insgesamt erweist sich die S/AS-Methode (unter Berücksichtigung der Fehlergrenzen) als ebenso gut geeignet ist wie die $J(J+1)$-Auftragung. Beide Temperaturverläufe stimmen qualitativ mit dem von T. Wassermann ermittelten überein jedoch ist der Temperaturanstieg, der bei Nutzung der vorherigen Apparatur bereits ab $4 \mathrm{~mm}$ einsetzt deutlich abrupter, ${ }^{9}$ was wahrscheinlich auf die in Ref. 9 verwendete Düsengeometrie von $8 \times 0.05 \mathrm{~mm}^{2}$ anstelle der hier verwendeten $4 \times 0.15 \mathrm{~mm}^{2}$ zurückzuführen ist. Ein zusätzlicher Effekt aufgrund der unterschiedlichen Hintergrunddrücke ist jedoch nicht auszuschließen. 


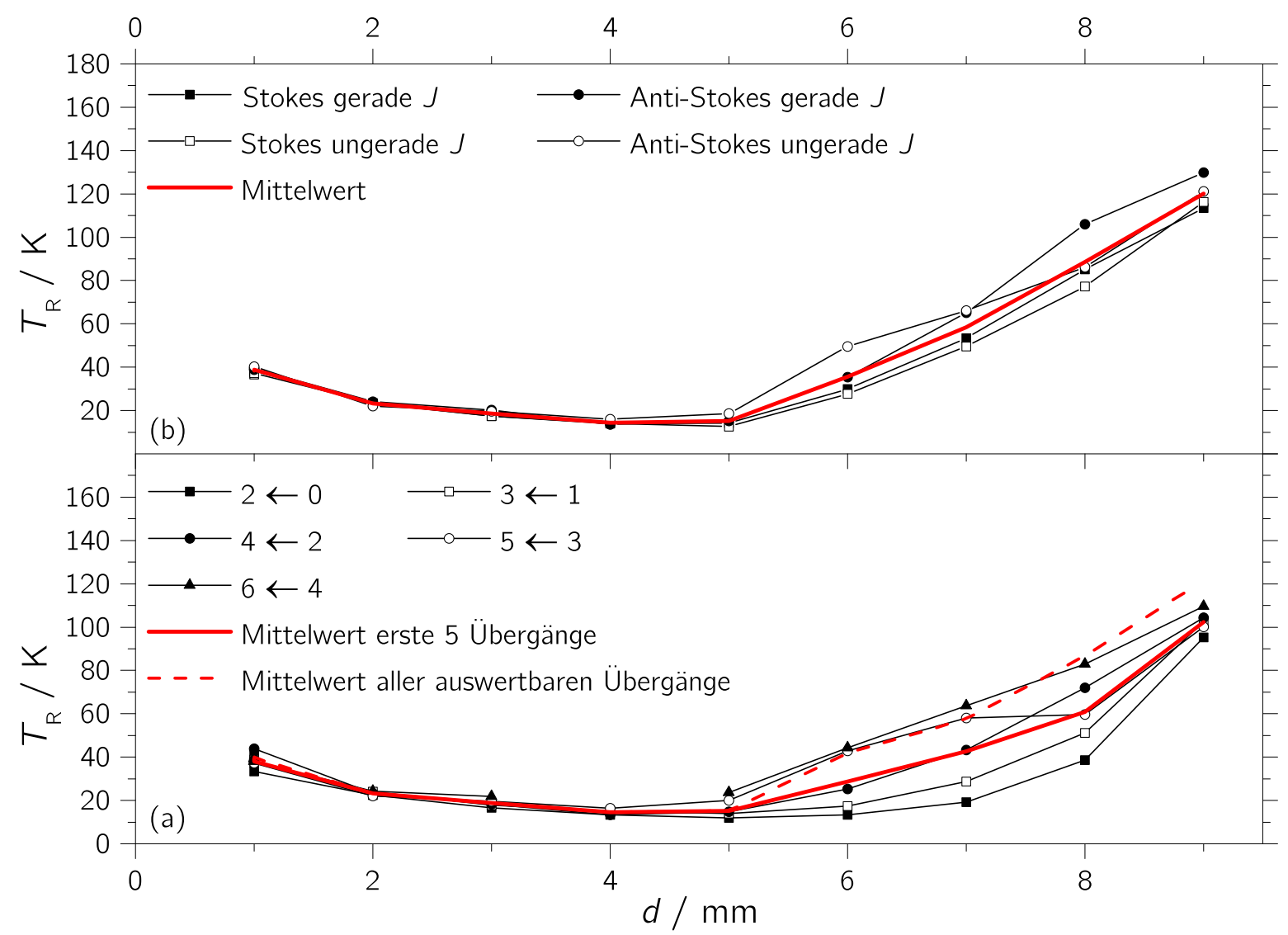

Abbildung 4.4: Temperaturverlauf für Stickstoff in der Überschallexpansion im Curry-Jet. (a) aus dem Stokes/Anti-Stokes-Intensitätsverhältnis bestimmte Rotationstemperaturen, (b) aus der Auftragung gegen $J(J+1)$ ermittelte Temperaturen. In (a) ist der Temperaturverlauf der ersten fünf Übergänge angegeben sowie deren Mittelwert, zudem auch der Mittelwert (rot) der Rotationstemperaturen aller auswertbaren Übergänge (rot, gestrichelt). In (b) sind die Temperaturverläufe, die jeweils aus dem Stokesund dem Anti-Stokes-Bereich ermittelt wurden, für gerade und ungerade Übergänge angegeben sowie deren Mittelwert (rot). 


\subsubsection{Referenzmessung Luft}

Als weitere Evaluation der Methode wurden Spektren von Luft bei Raumtemperatur aufgenommen. Einmal mit der Curry-Jet Apparatur und einmal mittels eines ReferenzRaman-Spektrometers, das eine bessere Auflösung besitzt. Außerdem kann hier durch die unempfindlichere CCD-Kamera auf den Einsatz eines Filters verzichtet werden. Luft wurde anstelle von Stickstoff verwendet, da beim Referenzspektrometer zurzeit keine Messungen anderer Substanzen möglich sind. Eine kurze Beschreibung des Spektrometers befindet sich im Anhang A.5.

Abbildung 4.5 zeigt zwei Spektren $(\mathrm{a}, \mathrm{b})$, die am Referenzspektrometer aufgenommen wurden. Einmal (a) wurde jeweils nur der Stokes- und anschließend der Anti-StokesBereich aufgenommen und einmal (b) wurden Stokes- und Anti-Stokes-Bereich gleichzeitig erfasst. Spektrum (c) zeigt eine am Curry-Jet durchgeführte Messung, wobei der Polfilter genutzt wurde. Die Auswertung der Banden erfolgte auch hier mittels Integration, wobei die stark überlappenden Linien, die aufgrund der schlechteren Auflösung am Curry-Jet nicht separiert werden konnten, nicht ausgewertet wurden. Die zur Auswertung genutzten Linien sowie die Zuordnung zu Sauerstoff und Stickstoff sind in Abbildung 4.5 im Stokes-Bereich mit der jeweiligen Rotationsquantenzahl $J$ des Ausgangsniveaus gekennzeichnet und zwar für Sauerstoff mit fett-gedruckten und für Stickstoff mit normal-gedruckten Zahlen. Die Zuordnung erfolgte für Stickstoff gemäß Ref. 74 und für Sauerstoff gemäß Ref. 76. In Spektrum (c) konnten nur sehr wenige Linien zur Auswertung genutzt werden, für Sauerstoff ergab sich hier lediglich ein Stokes-Anti-Stokes-Wertepaar. Dementsprechend konnte die Temperatur von Sauerstoff bei der gegebenen Auflösung nicht bestimmt werden.

Auch bei Spektrum (b) ist die Anzahl an Datenpunkten aufgrund des kleinen Messbereichs gering. Zudem sind im Anti-Stokes-Spektrum Artefakte zu erkennen, die möglicherweise zu verfälschten Intensitäten führen können, daher wurde auf die Auswertung der Linien, die direkt neben den Artefakten liegen verzichtet. Die Ergebnisse sind in Tabelle 4.3 dargestellt, wobei auch hier wieder die Mittelwerte aufgeführt sind. Als Fehler wurde für Stickstoff die Standardabweichung gewählt, bei Sauerstoff wurde für die S/AS-Methode die Standardabweichung und für die $J(J+1)$-Auftragung der Mittelwert der Fehler aus der linearen Regression gewählt, da hier nur zwei Temperaturwerte zur Verfügung standen. Es zeigt sich, dass sich aus den Curry-Jetspektren ähnliche Ergebnisse wie bei der Messung von Stickstoff in Helium ergeben. Bei den Messungen am Referenzspektrometer zeigt sich, dass besonders die Temperaturen aus Spektrum (a), die mit der S/AS-Methode bestimmt wurden stärker abweichen. Möglicherweise ist das auf unterschiedliche Pixelsensitivitäten zurückzuführen. Außerdem ist bei dem am Referenzspektrometer verwendeten Monochromator die polarisationsrichtungsspezifische Effizienz nicht bekannt und konnte somit nicht berücksichtigt werden. Fehlergrenzen und Mittelwerte sind aber vergleichbar mit den Stickstoff-Gasphasen aus Kapitel 4.1.1. 
Insgesamt zeigt sich, dass, solange einzelne Signale gefunden werden können, die separiert und eindeutig zuzuordnen sind, an der Curry-Jet Apparatur trotz relativ geringer Auflösung auch aus Substanzgemischen Rotationstemperaturen für Einzelkomponenten bestimmt werden können. Bei überlagerten Banden, wie hier für Sauerstoff in der Umgebungsluft gezeigt, sollte für eine Temperaturbestimmung jedoch ein Monochromator mit höherer Auflösung verwendet werden.

\begin{tabular}{llc}
\hline $\mathbf{N}_{2}$ & Methode & $\overline{T_{\text {rot }} / \mathrm{K}}$ \\
\hline Curry-Jet & $J(J+1)$ & $270 \pm 20$ \\
& $\mathrm{~S} / \mathrm{AS}$ & $320 \pm 20$ \\
Praktikum (S/AS gleichzeitig) & $J(J+1)$ & $270 \pm 50$ \\
& $\mathrm{~S} / \mathrm{AS}$ & $280 \pm 20$ \\
Praktikum (S/AS hintereinander) & $J(J+1)$ & $290 \pm 10$ \\
& $\mathrm{~S} / \mathrm{AS}$ & $260 \pm 50$ \\
\hline $\mathbf{O}_{2}$ & & \\
\hline Curry-Jet & $J(J+1)$ & - \\
& $\mathrm{S} / \mathrm{AS}$ & - \\
Praktikum (S/AS gleichzeitig) & $J(J+1)$ & $310 \pm 20$ \\
& $\mathrm{~S} / \mathrm{AS}$ & $280 \pm 20$ \\
Praktikum (S/AS hintereinander) & $J(J+1)$ & $300 \pm 10$ \\
& $\mathrm{~S} / \mathrm{AS}$ & $265 \pm 20$ \\
\hline
\end{tabular}

Tabelle 4.3: Mittlere Rotationstemperaturen von Stickstoff und Sauerstoff in der Gasphase bei ca. $300 \mathrm{~K}$, die mit dem Curry-Jetspektrometer sowie mit dem Referenzspektrometer (Praktikum) bestimmt wurden. Beim Referenzspektrometer wurde einmal der Stokes- und Anti-Stokes-Bereich gleichzeitig gemessen (Spektrum (b) Abb. 4.5) und einmal die beiden Bereiche hintereinander (Spektrum (a) Abb. 4.5). 


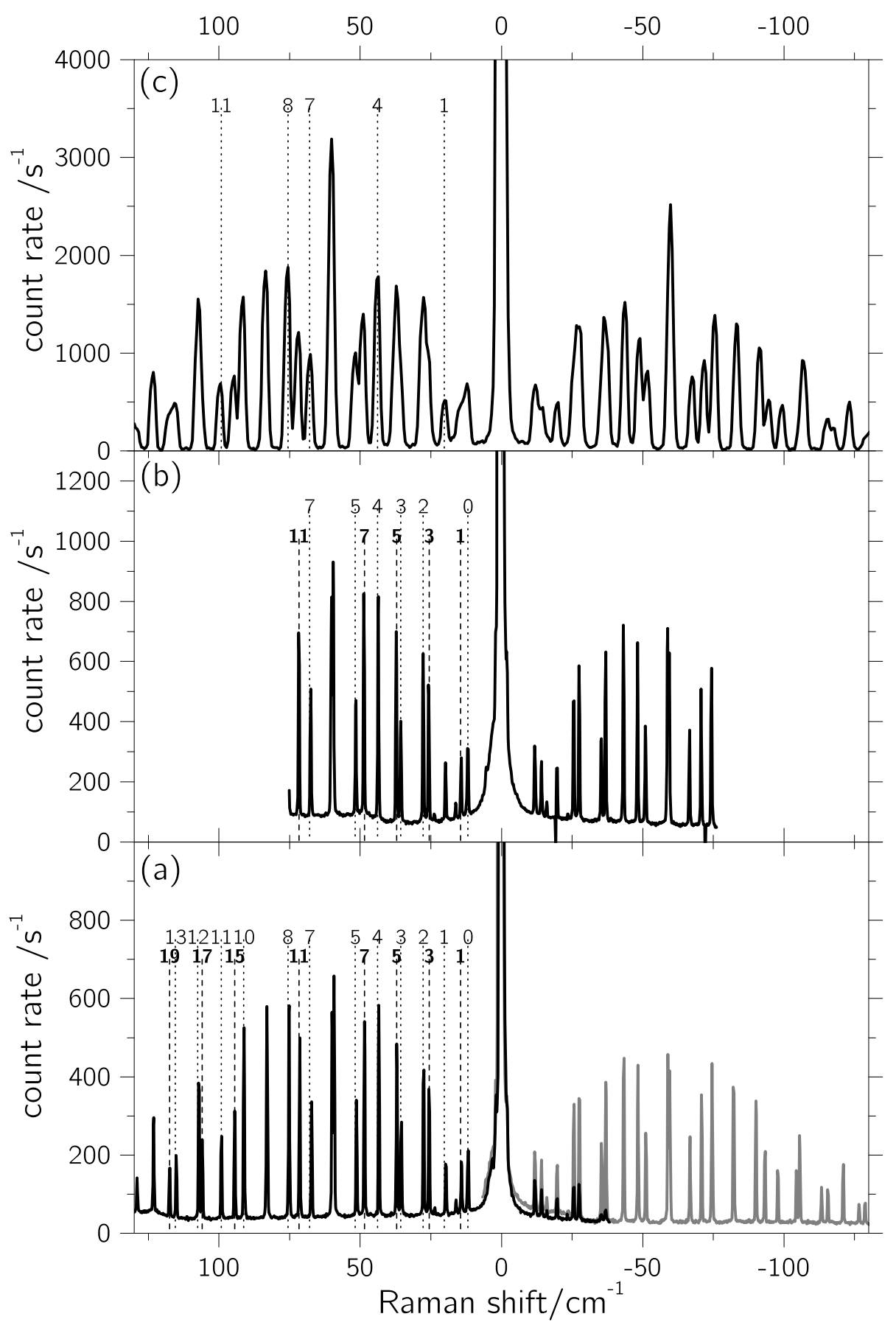

Abbildung 4.5: Spektren von Luft (a,b) am Referenzspektrometer ohne Filter und (c) am Curry-Jetspektrometer mit Polfilter aufgenommen. (a) schwarz: Stokes, grau: AntiStokes, (b,c) Stokes und Anti-Stokes gleichzeitig aufgenommen. Im Stokes-Bereich sind jeweils diejenigen Linien mit der Rotationsquantenzahl $J$ des Ausgangsniveaus gekennzeichnet, die zur Auswertung verwendet wurden $\left(\right.$ Fett: $\mathrm{O}_{2}$, normal: $\left.\mathrm{N}_{2}\right)$. Details zu den Messbedingungen finden sich im Anhang A.3. 


\subsubsection{Rotationstemperatur von Wasser im Jet}

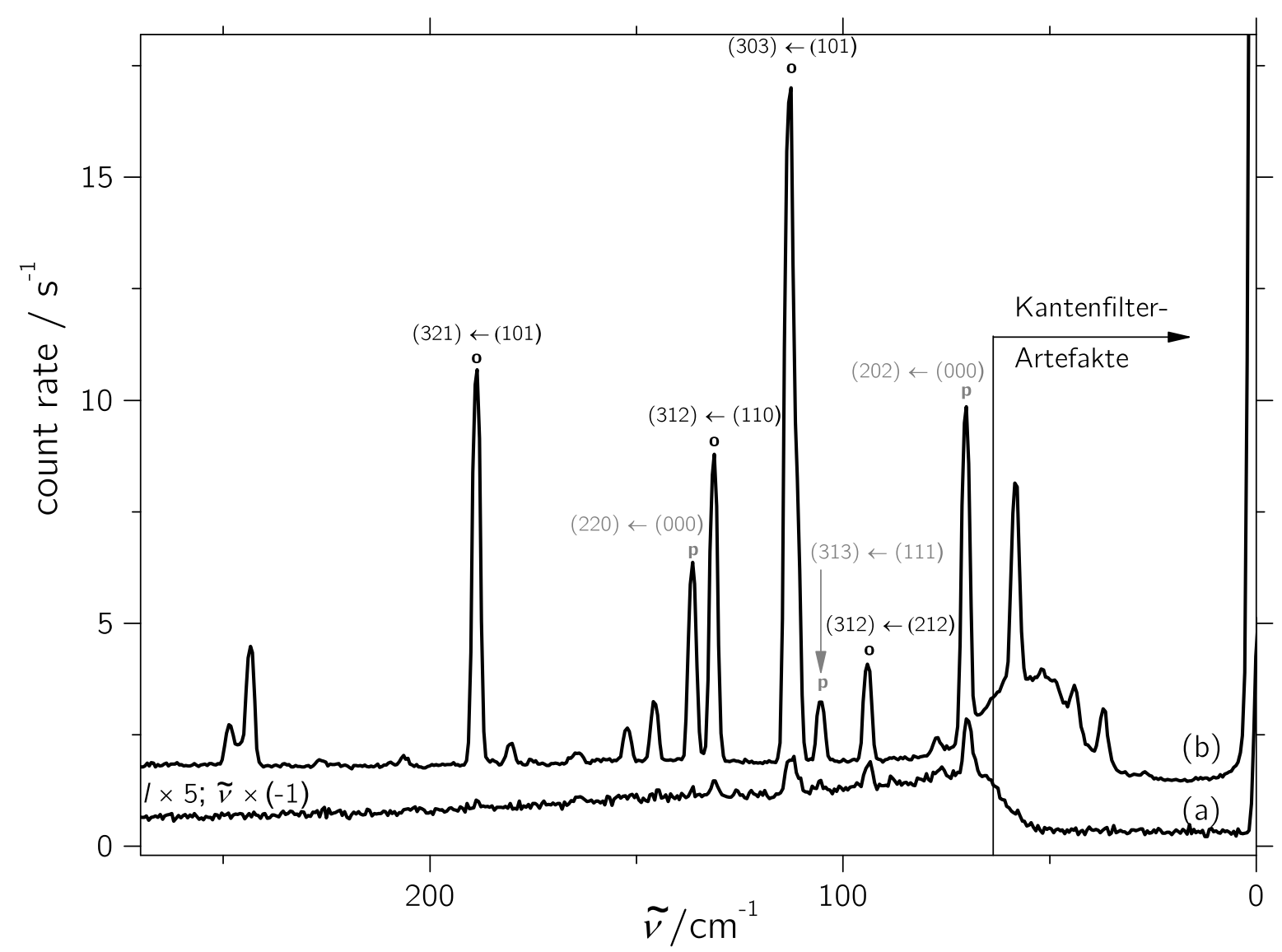

Abbildung 4.6: Exemplarische Anti-Stokes (a) und Stokes (b) Spektren der Wasserrotationslinien. Es wurde eine Expansion von Wasser in Helium durchgeführt. Die zur Temperaturbestimmung verwendeten Linien sind durch den entsprechenden StokesÜbergang mit den Rotationsquantenzahlen $\left(J K_{\mathrm{a}} K_{\mathrm{c}}\right)$ und Kernspinisomere mit ortho (o) (schwarz) und para (p) (grau) gekennzeichnet. ${ }^{77}$ Die Intensität des Anti-Stokes Spektrums ist mit einem Faktor von 5 und die Wellenzahlen mit einem Faktor von (-1) skaliert. Zudem ist der Bereich, ab dem eine Auswertung aufgrund der Kantenfilter nicht mehr möglich ist, angegeben. Details zu den Messbedingungen finden sich im Anhang A.3.

Ein komplexeres System, welches sich zum Test der S/AS-Methode eignet ist das Wasser-Monomer. Da es sich um einen asymmetrischen Kreisel handelt ist eine einfache Analyse mittels $J(J+1)$-Auftragung nicht möglich. Außerdem muss auch hier wie bei Stickstoff zwischen den beiden Kernspinisomeren unterschieden werden. Die Rotationstemperatur von Wasser in Überschallexpansionen wurde bereits in warmen Expansionen durch Vergleich mit simulierten Monomer-Spektren abgeschätzt. ${ }^{57,58} \mathrm{Au}$ ßerdem wurde sie aus Raman-Schwingungsrotationsübergängen bestimmt. ${ }^{78}$ Hierbei ist 
allerdings eine Kombination mit theoretischen Werten für die Streuquerschnitte ${ }^{79}$ notwendig. Zudem konnten nur sehr milde Expansionsbedingungen verwendet werden, da ansonsten Überlagerungen mit Clusterbanden auftreten. ${ }^{78}$ Dieses Problem ist für reine Rotationsspektren geringer, da die Streuquerschnitte der niederfrequenten Schwingungsbanden der Cluster gering im Vergleich zu denen der Monomer-Rotationsübergänge sind und zudem die Anzahl an Clustern im Vergleich zum Monomer sehr klein ist. Wasser sollte zunächst als Gasphase im thermischen Gleichgewicht gemessen werden, allerdings konnte aufgrund von Undichtigkeiten der Apparatur kein reines Wasserspektrum aufgenommen werden. Die Überlagerung mit den Rotationslinien von Stickstoff und Sauerstoff aus der Umgebungsluft war zu stark für eine entsprechende Auswertung. Des Weiteren war es nicht möglich einen Polfilter einzusetzen, da die Messzeiten für ein auswertbares Spektrum zu lang waren und der Detektor sonst wahrscheinlich Schaden genommen hätte. Daher wurden alle Spektren unter Nutzung der Kantenfilter aufgenommen.

Die zur Auswertung verwendeten Stokes- und Anti-Stokes-Jetspektren wurden in einem Abstand von $2 \mathrm{~mm}$ zur Düse aufgenommen. Abbildung 4.6 zeigt beispielhaft ein Anti-Stokes- (a) und ein Stokes-Spektrum (b), wobei bei ersterem die Wellenzahlen mit (-1) und die Intensitäten mit 5 multipliziert wurden. Die zur Auswertung verwendeten Rotationslinien sind jeweils mit ortho (o) und para (p) sowie durch den jeweiligen Stokes-Übergang mit den Rotationsquantenzahlen $\left(J K_{\mathrm{a}} K_{\mathrm{c}}\right)$ gekennzeichnet, wobei die Zuordnung gemäß Ref. 77 erfolgte. In Tabelle A.5.8 im Anhang sind die relativen Intensitäten und die bestimmten Temperaturen aufgeführt. Auch hier wurden die Intensitäten durch Integration bestimmt und die Anti-Stokes-Integrale gemäß der Monochromator- und Filterfehler korrigiert. Die Korrektur führt allerdings lediglich zu einer Erhöhung der effektiven Temperaturen von maximal $2 \mathrm{~K}$ und ist somit vernachlässigbar.

Es wurden drei Messungen mit einer Düsentemperatur von $25^{\circ} \mathrm{C}$ sowie je eine bei einer Düsentemperatur von $80^{\circ} \mathrm{C}$ und $120^{\circ} \mathrm{C}$ durchgeführt. Alle Expansionen wurden mit $100 \%$ Helium als Trägergas, einem Düsenabstand von $2 \mathrm{~mm}$, einem Stagnationsdruck von 0.7 bar sowie einer Sättigertemperatur von $18^{\circ} \mathrm{C}$ durchgeführt. Die Messzeiten betrugen für alle Stokes-Spektren $18 \times 100 \mathrm{~s}$ und für alle Anti-Stokes-Spektren $18 \times 200 \mathrm{~s}$. Abbildung 4.7 zeigt ein Energieniveauschema der betrachteten Rotationsniveaus von Wasser, ${ }^{15}$ die Energieniveaus wurden aus Ref. 77 übernommen. Die in der Grafik angegebenen Werte unterscheiden sich um bis zu $9 \mathrm{~K}$ von denen in Ref. 15, aufgrund der in Kapitel 3.4 beschriebenen Auswertungsproblematik. Die verschiedenen Übergänge sind als Pfeile eingezeichnet und die jeweiligen Rotationstemperaturbereiche, die für eine Düsentemperatur von $25^{\circ} \mathrm{C}$ bestimmt wurden, sind angegeben. Es zeigt sich, dass die Temperaturen für ortho- und para-Wasser sich im Rahmen der Messgenauigkeit nicht unterscheiden. Die Werte liegen zwischen $30 \mathrm{~K}$ und $58 \mathrm{~K}$. Mit ca. 30-60 K ergeben sich hier also vergleichbare Werte wie für Stickstoff. Für die Expansionen bei $80^{\circ} \mathrm{C}$ und $120^{\circ} \mathrm{C}$ Düsentemperatur wurden Rotationstemperaturen von $42-61 \mathrm{~K}$ bzw. 
38-54 K ermittelt. Die Erhöhung der Düsentemperatur führt also bei einem Abstand von $2 \mathrm{~mm}$ keine signifikanten Veränderungen der Monomer-Rotationstemperaturen von Wasser herbei. Dieser Befund ist relativ plausibel, denn bei den Expansionen wurde ein relativ großer Anteil von Wasser im Trägergas gewählt. Hierdurch kommt es bei Expansion besonders bei niedriger Düsentemperatur zu vermehrter Clusterbildung, wobei die Kondensationswärme teilweise von den verbleibenden Monomeren abgeführt wird. Bei Erhöhung der Düsentemperatur ergibt sich einerseits eine höhere Temperatur der Monomere vor der Expansion, andererseits muss aber weniger Kondensationswärme abgeführt werden, da sich eine geringere Anzahl an Clustern ausbildet, so dass sich die Monomer-Temperatur insgesamt wenig verändert.

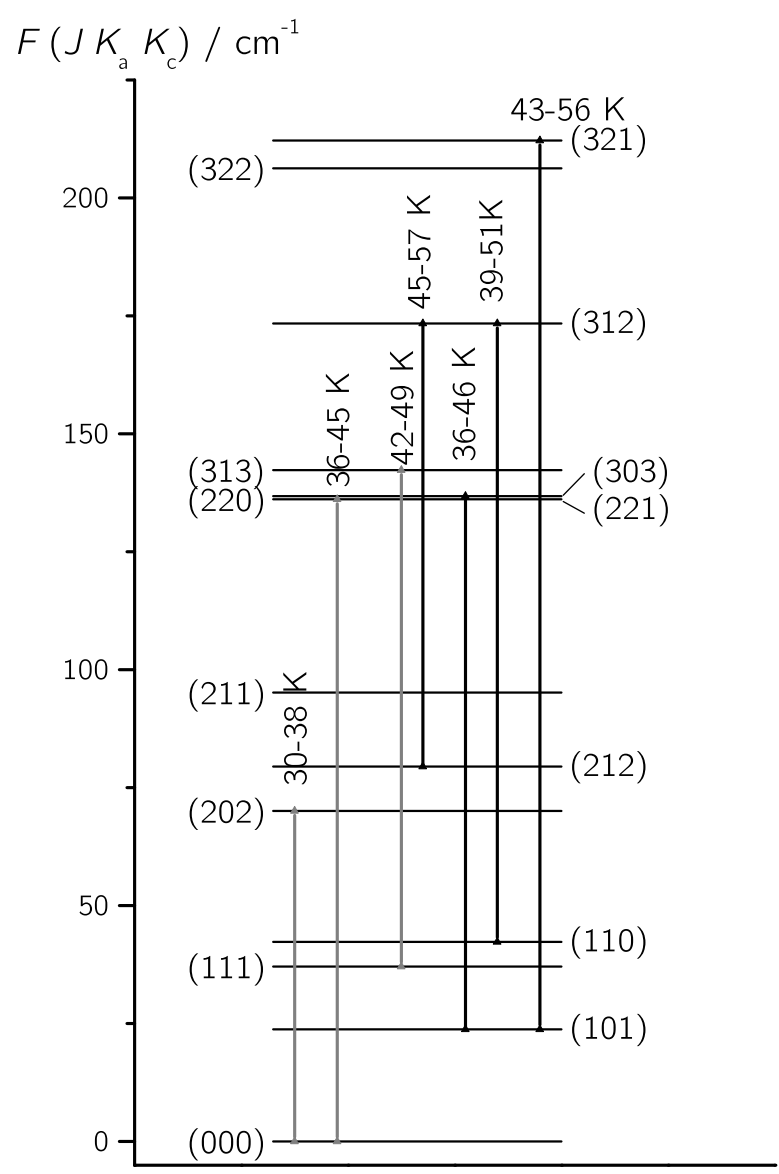

Abbildung 4.7: Schematische Darstellung der Rotationsniveaus von Wasser. ${ }^{15}$ Die ausgewerteten Rotationsübergänge und deren Rotationstemperaturbereiche für eine Düsentemperatur von $25^{\circ} \mathrm{C}$ sind gezeigt. ortho-Übergänge sind in schwarz und paraÜbergänge in grau dargestellt. 


\subsection{Schwingungstemperaturen am Beispiel von 2,2,2-Trifluorethanol}

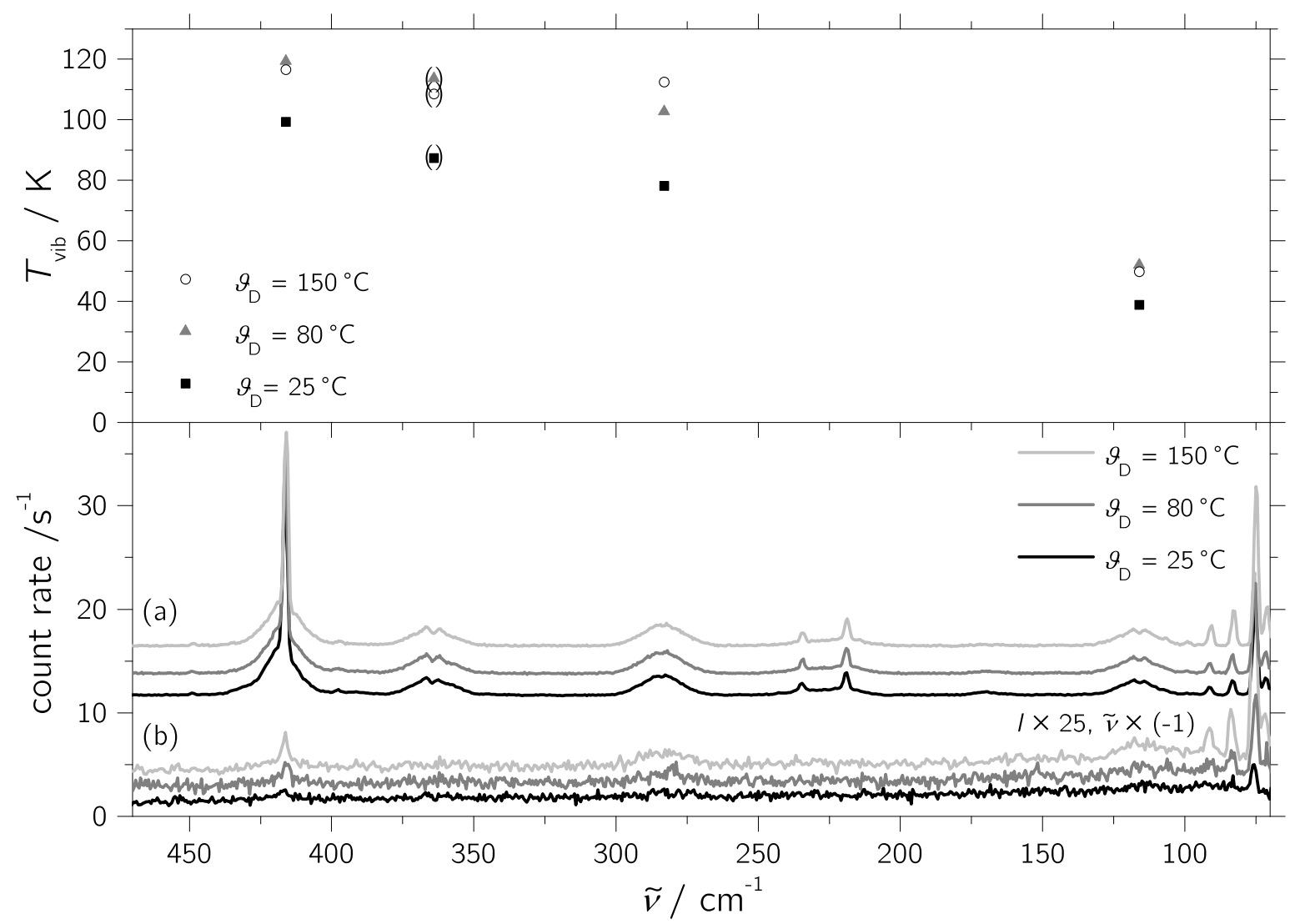

Abbildung 4.8: Unten: Jeweils drei Stokes- (a) und Anti-Stokes-Spektren (b) von 2,2,2Trifluorethanol in Helium in einer Jetexpansion mit einem Abstand von $1 \mathrm{~mm}$ zur Düse. Die Intensitäten der Anti-Stokes-Spektren (untere drei) wurden mit einem Faktor von 25 skaliert und die Wellenzahlen mit $(-1)$. Die Düsentemperaturen $\left(\vartheta_{\mathrm{D}}\right)$ betrugen $25^{\circ} \mathrm{C}$ (schwarz), $80{ }^{\circ} \mathrm{C}$ (dunkelgrau) und $150^{\circ} \mathrm{C}$ (hellgrau) (weitere Details über die Aufnahmeparameter siehe Spektrenverzeichnis A.3). Oben: Die aus den einzelnen Schwingungsbanden mittels des Stokes/Anti-Stokes-Intensitätsverhältnisses bestimmten Schwingungstemperaturen. Eingeklammerte Symbole sind Werte, die aus nur bedingt auswertbaren Banden erhalten wurden.

Als weitere Anwendung wurden Schwingungstemperaturen bestimmt. 2,2,2-Trifluorethanol wurde verwendet, da es eine Reihe von niederfrequenten Schwingungsmoden besitzt und außerdem weil es in nur einer Monomer-Konformation (gauche) im Jet vorliegt. ${ }^{60}$ Auch hier wurden die Kantenfilter verwendet. Als erster Test wurden Schwingungstemperaturen der Gasphase im thermischen Gleichgewicht bestimmt. Hier ergaben 
sich Werte von $280 \pm 10 \mathrm{~K}$, die relativ gut mit der Umgebungstemperatur von $300 \mathrm{~K}$ übereinstimmen.

Für die Jetspektren wurden Bedingungen gewählt, bei denen selbst für niedrige Düsentemperaturen nur sehr wenige Cluster vorlagen, also eine niedrige Sättigertemperatur von $-25^{\circ} \mathrm{C}$ und ein relativ geringer Abstand zur Düse von $1 \mathrm{~mm}$. Als Sonde hierfür wurden die OH-Streckschwingungsbanden der Cluster genutzt. Abbildung 4.8 unten zeigt Stokes- (oben) und Anti-Stokes-Spektren (unten) für Düsentemperaturen von $25^{\circ} \mathrm{C}$ (schwarz), $80^{\circ} \mathrm{C}$ (dunkelgrau) und $150^{\circ} \mathrm{C}$ (hellgrau). In den Stokes-Spektren sind sechs Banden erkennbar, die in Tabelle 4.4 aufgelistet sind. Die Zuordnung erfolgte mithilfe von Depolarisationsexperimenten und durch Vergleich mit quantenchemischen Rechnungen. Neben den Banden des Trifluorethanols sind unterhalb von $100 \mathrm{~cm}^{-1}$ außerdem noch Rotationslinien von Sauerstoff und Stickstoff, die von Verunreinigungen mit Luft stammen, vorhanden. Im oberen Bereich der Abbildung sind die jeweils bestimmten Schwingungstemperaturen gezeigt. Die Intensitäten wurden per Integration bestimmt und durch Variation der Integrationsgrenzen wurde der Fehler der Temperaturen auf ca. $20 \mathrm{~K}$ abgeschätzt. Es zeigt sich, dass die drei Banden oberhalb von $250 \mathrm{~cm}^{-1}$ auf Temperaturen von 80-120 K relaxieren während die einzige auswertbare Bande (Torsion) unterhalb auf ca. $50 \mathrm{~K}$ relaxiert. Die für die Bande bei $364 \mathrm{~cm}^{-1}$ bestimmten Temperaturen können nur bedingt in Betracht gezogen werden, da sie im Anti-Stokes-Spektrum kaum auswertbar ist. Auffällig ist, dass die zwei Banden bei 218 und $235 \mathrm{~cm}^{-1}$, nicht im Anti-Stokes-Spektrum wiedergefunden werden können. Das legt nahe, dass Moleküle in den zugehörigen angeregten Zuständen sehr stark relaxieren. Um diese Vermutung zu bestätigen wurde in Abbildung 4.9 ein Anti-Stokes-Spektrum simuliert (c). Aufgrund des vergleichsweise guten Signal-zu-Rausch-Verhältnisses wurden die Spektren, die mit einer Düsentemperatur von $150^{\circ} \mathrm{C}$ aufgenommen wurden, verwendet. Die für die verschiedenen Banden ermittelten Temperaturen wurden auf Zehnerschritte gerundet und aus der Bandenposition und der Temperatur gemäß Gleichung 2.12 das theoretische Anti-Stokes/Stokes-Intensitätsverhältnis bestimmt. Die Werte befinden sich in Tabelle

\begin{tabular}{ll}
\hline$\widetilde{\nu} / \mathrm{cm}^{-1}$ & Zuordnung \\
\hline 116 & $\tau_{\mathrm{CF}_{3}}$ \\
218 & Fermiresonanz: $\delta_{\mathrm{CCO}}+$ \\
235 & $2 \tau_{\mathrm{CF}_{3}}$ \\
283 & $\tau_{\mathrm{OH}}$ \\
364 & $\gamma_{\mathrm{CF}_{3}} / \tau_{\mathrm{OH}}$ \\
416 & $\delta_{\mathrm{CCO}} / \tau_{\mathrm{OH}}$ \\
\hline
\end{tabular}

Tabelle 4.4: Niederfrequente Schwingungsbanden von 2,2,2-Trifluorethanol inklusive Zuordnung in Übereinstimmung mit Ref. 60 und 80 sowie mit quantenchemischen Rechnungen (vgl. auch Kap. 6). Hierbei beschreibt $\tau$ eine Torsions-, $\delta$ eine Deformations- und $\gamma$ eine Schaukelschwingung. 


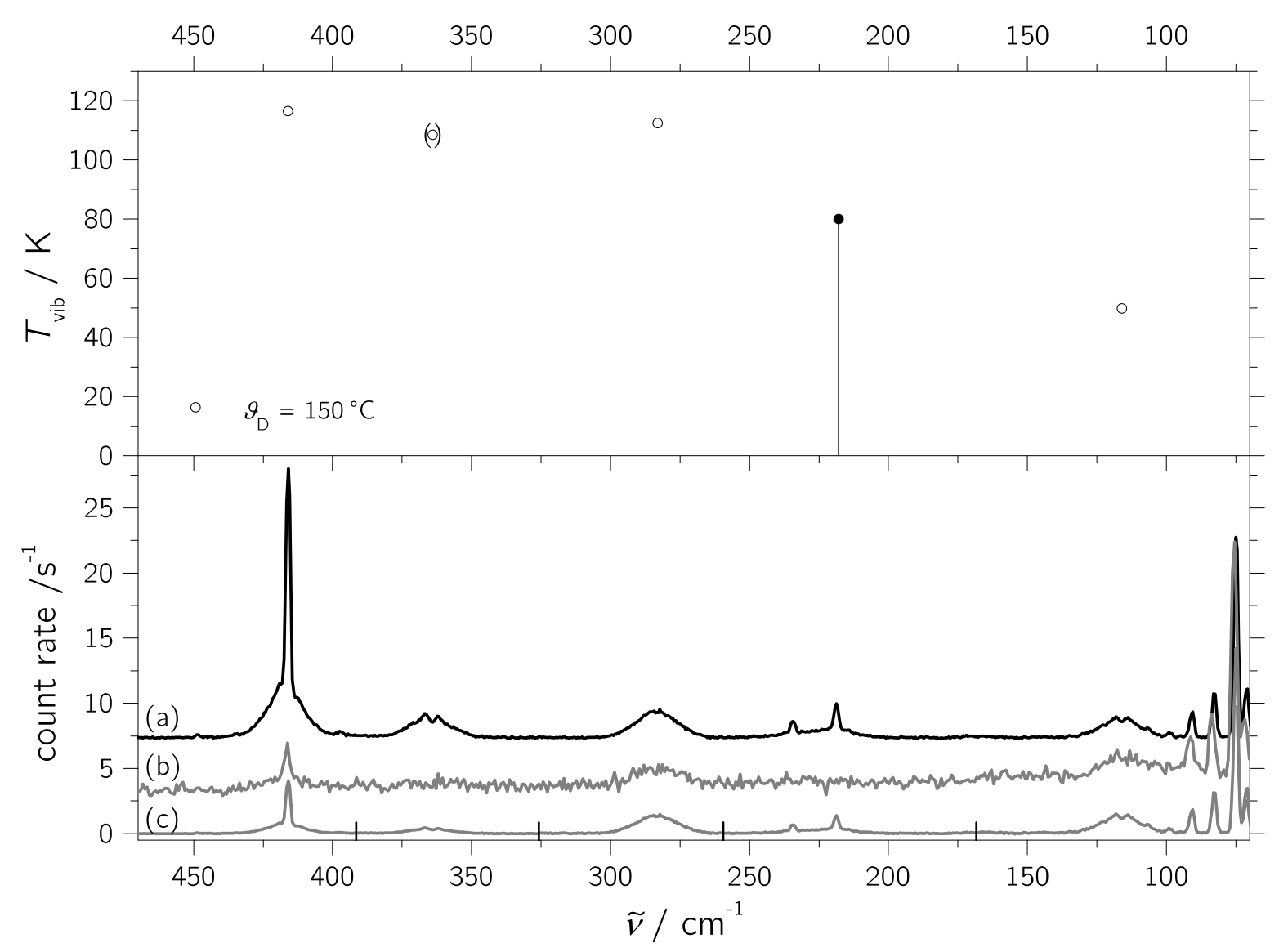

Abbildung 4.9: Oben: Experimentell bestimmte Schwingungstemperaturen bei $\vartheta_{\mathrm{D}}=150^{\circ} \mathrm{C}$ und aus einer Simulation abgeschätztes Schwingungstemperaturmaximum (Bande $218 \mathrm{~cm}^{-1}$ ). Unten: (a) Stokes Spektrum $\vartheta_{\mathrm{D}}=150^{\circ} \mathrm{C}$, (b) Anti-Stokes-Spektrum $\vartheta_{\mathrm{D}}=150^{\circ} \mathrm{C}$, Intensitäten mit einem Faktor von 25 multipliziert und (c) aus (a) abschnittsweise simuliertes Anti-Stokes-Spektrum (Intensitäten sind ebenfalls mit Faktor 25 multipliziert). Aus den (gerundeten) bestimmten Temperaturen wurde für jede Bande das theoretische Intensitätsverhältnis bestimmt und als Dämpfungsfaktor eingesetzt. Die verwendeten Werte befinden sich in Tabelle A.5.10 im Anhang. Die einzelnen Abschnitte sind durch senkrechte Striche voneinander getrennt. Es zeigt sich, dass auf diese Weise für die experimentell nicht auswertbare Bande bei $218 \mathrm{~cm}^{-1}$ die Schwingungstemperatur auf $<80 \mathrm{~K}$ abgeschätzt werden kann. 
A.5.10 im Anhang. Die ermittelten Werte können nun als Dämpfungsfaktoren verwendet werden um so aus dem Stokes-Spektrum, das im Vergleich zum gemessenen Anti-Stokes-Spektrum ein sehr viel besseres S/N-Verhältnis besitzt, ein Anti-StokesSpektrum zu berechnen. Hierzu wird das Stokes-Spektrum abschnittweise mit den ermittelten Faktoren multipliziert. Das Ergebnisspektrum ist in Abbildung 4.9 (c) zu sehen, wobei das Spektrum genau wie das gemessene Spektrum zur besseren Sichtbarkeit mit einem Faktor von 25 multipliziert wurde.

Ein Vergleich von Spektrum (b) und (c) zeigt für die bereits ausgewerteten Banden eine gute Übereinstimmung. Für die nicht auswertbaren Banden wurde eine maximale Temperatur von $80 \mathrm{~K}$ angenommen. (Es wurde für beide Banden derselbe Faktor, der für $218 \mathrm{~cm}^{-1}$ ermittelt wurde, eingesetzt, da die Bereiche nicht eindeutig separierbar sind.) Bei dieser Temperatur müsste allerdings zumindest der Peak bei $218 \mathrm{~cm}^{-1}$ noch im Rauschen erkennbar sein, dementsprechend muss die Bande auf eine Schwingungstemperatur $<80 \mathrm{~K}$ im Jet relaxieren. Interessant wären an dieser Stelle weitere Studien von Banden unterhalb von $250 \mathrm{~cm}^{-1}$ um genauer zu ergründen, ob es einen linearen Abfall der Schwingungstemperaturen ab diesem Wert gibt oder aber eher einen Sprung. Außerdem könnte möglicherweise durch die Untersuchung anderer Systeme herausgefunden werden, ob die stärkere Relaxation der zwei Banden in Zusammenhang mit der Fermiresonanz zwischen ihnen steht. Ebenfalls denkbar ist, dass $\mathrm{CF}_{3}$-Torsionsschwingungen grundsätzlich eher wie Rotationsfreiheitsgrade relaxieren, während die in eine schwache Wasserstoffbrücke eingebundene $\mathrm{OH}$-Torsion offenbar eher ein normales Relaxationsverhalten zeigt. 


\subsection{Zusammenfassung und Ausblick}

Die Temperaturbestimmung aus dem Stokes- und Anti-Stokes-Intensitätsverhältnis wurde systematisch für Gasphasen- und Jetspektren erprobt. Sie konnte anhand verschiedener Messmodi unter Nutzung des Testsystems Stickstoff als geeignet evaluiert werden.

Anhand der weiteren Testsysteme Wasser und 2,2,2-Trifluorethanol konnten Rotationstemperaturen von 30-60 K und Schwingungstemperaturen von ca. $100 \mathrm{~K}$ ermittelt werden. Die errechneten Werte stimmen qualitativ gut mit bereits publizierten Daten anderer Systeme unter vergleichbaren Expansionsbedingungen überein. ${ }^{67}$ Erste Hinweise auf einen Übergang zwischen den höheren Schwingungstemperaturen und den tieferen Rotationstemperaturen bei besonders niedrigen Schwingungsfrequenzen und Torsionsbewegungen konnten gefunden werden.

Die Methode stützt sich rein auf experimentelle Werte. Es ist keine Kenntnis von Übergangsmomenten oder spektroskopischen Zuordnungen notwendig. Außerdem ist es möglich die Temperaturentwicklung einzelner Rotations- oder Schwingungsübergänge nachzuverfolgen. Der größte Nachteil der Methode besteht in der zurzeit verwendeten Messanordnung, die es nicht ermöglicht Stokes- und Anti-Stokes-Bereich gleichzeitig aufzunehmen. Durch den Wechsel der verwendeten Filter entstehen erhebliche Einbußen in der Signalstärke, die zu relativ großen Fehlerintervallen führen. Durch den Einsatz eines sehr schmalbandigen Kerbfilters oder aber durch den Einsatz von Kantenfiltern im kollimierten Strahlengang könnten die Fehlergrenzen deutlich verkleinert werden. Mit diesen Verbesserungen wäre es denkbar routinemäßig für Systeme, die niederfrequente Banden besitzen, zunächst eine Temperaturbestimmung durchzuführen um die jeweilige Expansion genauer charakterisieren zu können.

Eine Anwendung auf Aggregate ist wahrscheinlich nur für Systeme möglich, bei denen bereits in der Gasphase unter Gleichgewichtsbedingungen eine hohe Anzahl an Clustern vorliegt, wie z.B. bei Ameisensäure, da ansonsten die Konzentration zu gering ist um ein auswertbares Anti-Stokes-Spektrum zu erhalten.

Ein nächster Schritt könnte die Anwendung der Methode zur Untersuchung des Temperaturverlaufs der Schwingungsniveaus in Abhängigkeit vom Abstand zur Düse sein. Außerdem sollten weitere Systeme besonders im Bereich von $50-300 \mathrm{~cm}^{-1}$ untersucht werden um den in den vorliegenden Ergebnissen angedeuteten Temperaturabfall genauer zu analysieren. 


\section{Raman-Spektroskopie kleiner Wasseraggregate}

\subsection{Einleitung}

Wasser ist eines der häufigsten Moleküle der Erdoberfläche und von fundamentaler Bedeutung für die Entstehung des Lebens, was mitunter auf seine vielfältigen anomalen Eigenschaften zurückzuführen ist. ${ }^{81}$ Die Anomalien können qualitativ durch die Wasserstoffbrückenbindungen, die als stärkste Wechselwirkungen zwischen den Molekülen auftreten, verstanden werden. ${ }^{81}$ Zum Verständnis der Struktur und Dynamik in Wasser ist die Schwingungsspektroskopie aufgrund ihrer Empfindlichkeit gegenüber der Wasserstoffbrücken-Umgebung gut geeignet. ${ }^{82}$ Besonders relevant für biologische Prozesse ist die Energieübertragung in der Flüssigkeit. ${ }^{83}$ Die ultraschnelle Energieumverteilung in flüssigem Wasser, die im Fall der OH-Streckschwingungsanregung auf einer Zeitskala von 70 fs stattfindet, ${ }^{84,85}$ ist daher Gegenstand zahlreicher fs-Experimente. ${ }^{84,86}$

Zur Modellierung von Wasserstoffbrückennetzwerken in der Flüssigkeit wird häufig der Clusteransatz verwendet, ${ }^{1,87}$ bei dem die Moleküle Schritt für Schritt zu größeren Aggregaten zusammengefügt werden. Auf diese Weise können Veränderungen der Eigenschaften beim Übergang von der Gasphase zur Flüssigkeit untersucht werden. ${ }^{1}$ Das detaillierte Verständnis der kleinen Aggregate kann dann genutzt werden um theoretische Modelle zu entwickeln, die auf die komplexeren kondensierten Systeme übertragen werden können. ${ }^{1}$ Raman-Studien an Wasser wurden bereits in den 1960er Jahren von Walrafen et al. ${ }^{88}$ durchgeführt. Der flüssige Aggregatzustamd wurde hinsichtlich intermolekularer Schwingungen untersucht. Es konnten verschiedene Librationsschwingungen sowie Streckschwingungen der wasserstoffbrückengebundenen H-Atome zugeordnet werden. Erste Vermutungen über die Struktur der enthaltenen „Wassercluster“ ergaben ein $C_{2}$ symmetrisches Pentamer. ${ }^{88}$

Durch die Entwicklung verschiedener Clustererzeugungsmethoden existieren heute vielfältige Studien kleiner Wassercluster. Neben Matrixisolationsspektrokopie ${ }^{89,90}$ und der Studie der Aggregate mittels Chromophorenmarkierung ${ }^{53,91}$ ist vor allem die Untersuchung isolierter und unmarkierter Cluster interessant, da diese besonders gut mit theoretischen Modellen ${ }^{92}$ vergleichbar sind. Es existieren zahlreiche Infrarot- ${ }^{20-22,87}$ und 
Mikrowellen-spektroskopische ${ }^{93}$ Studien, durch die bereits detaillierte Informationen zu Kernquanteneffekten und elektronischer Kooperativität verfügbar sind.

Hingegen existieren nur wenige Raman-Spektren kleiner, isolierter Wassercluster, wodurch ein, besonders für das Verständnis der cyclischen Aggregate, wichtiger Teil an komplementären Daten bislang fehlte. Eine erste CARS-Studie zu Wasserclustern in Überschallexpansionen wurde von Wuelfert et al. veröffentlicht ${ }^{94}$ und mit Nelander et al. kontrovers diskutiert. ${ }^{95,96}$ Eine sichere Zuordnung des dort gefundenen Signals ist aufgrund der nicht-linearen Intensitätsentwicklung mit der Clustergröße im CARS-Experiment schwierig. Eine Analyse der Kopplung zu benachbarten Oszillatoren in Wasserclustern, die den Energiefluss nach lokaler Anregung bestimmt, blieb daher bislang aus. $^{15}$

Die in dieser Arbeit vorgestellten Ergebnisse ermöglichen diese Kopplungsanalyse für die OH-Streckschwingungen der cyclischen Wassercluster bis hin zum Pentamer. Hierzu werden die in Kapitel 2.4 vorgestellten Hückel-artigen Kopplungsmatrizen ${ }^{91}$ verwendet. Die Energieaufspaltung der entarteten Monomerschwingungen in symmetrische und asymmetrische Linearkombinationen erfolgt entweder durch eine Kopplung über die Bindungen oder durch den Raum. ${ }^{15}$

Das hierdurch entstehende Muster kann in der Frequenzdomäne beobachtet werden ${ }^{97}$ anschaulicher $\mathrm{zu}$ verstehen ist es allerdings in der Zeitdomäne. Bei Anregung eines lokalen Oszillators mittels eines ultrakurzen Laserpulses würde die Energie sehr schnell zwischen den gekoppelten Oszillatoren delokalisiert werden. Im Falle der cyclischen Aggregate handelt es sich unter der Annahme, dass es keine weiteren Verteilungskanäle gibt, um einen periodischen Prozess, bei dem sich die Energie nach einer gewissen Zeit wieder im ursprünglichen Oszillator konzentriert. ${ }^{15}$ Hierbei sind die Größe der Aufspaltung und die Geschwindigkeit des Energieabflusses proportional miteinander verknüpft.

Bis zu einem gewissen Grad kann dieses Modell auch auf die flüssige und feste Phase, bei denen die Energieumverteilung irreversibel ist, übertragen werden, da der erste Schritt der Umverteilung eine Energieübertragung zwischen zwei benachbarten, gekoppelten Oszillatoren darstellt. Somit ist die Größe der Kopplungskonstante zwischen den OH-Streckoszillatoren ein wichtiger Parameter zum Verständnis der fs-Dynamik in der Flüssigkeit. Für die Flüssigkeit wurde die Kopplungskonstante bereits auf ca. $12 \mathrm{~cm}^{-1}$ abgeschätzt. ${ }^{86}$ Neueste Modellrechnungen kleiner Cluster sagen eine ähnliche

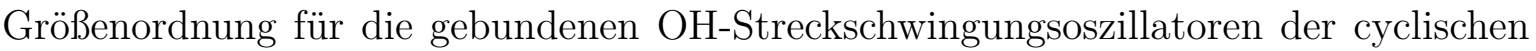
Aggregate voraus. ${ }^{98}$ Allerdings ist dieser Wert sehr viel kleiner als experimentelle Werte von $20-30 \mathrm{~cm}^{-1}$, die für Alkoholcluster mit derselben Wasserstoffbrückentopologie bestimmt wurden. ${ }^{12}$ Eine nun mögliche Kopplungsanalyse kleiner Wassercluster aus experimentellen Spektren soll im Folgenden diese Diskrepanz näher untersuchen. 
Die Zuordnung der erhaltenen Signale ist für die verwendete Messanordnung besonders für die cyclischen Aggregate sicher möglich, da die Clustersignale aufgrund der mit steigender Clustergröße stärker werdenden Kooperativität und der hierdurch größer werdenden Rotverschiebung gegenüber dem Monomer gut separiert sind. Durch Aufnahme entsprechender $\mathrm{D}_{2} \mathrm{O}$-Spektren kann die Zuordnung weiterhin bestätigt werden, so dass auf eine größenselektive Methode verzichtet werden kann. Ein Großteil der im folgenden Kapitel vorgestellten Ergebnisse ist bereits in Ref. 15 veröffentlicht.

\subsection{Jetexperimente im $\mathrm{OH}-S t r e c k s c h w i n g u n g s b e r e i c h$}

Bereits von P. Zielke und Z. Xue wurden Messungen von Wasser/Trägergas-Expansionen an der Curry-Jet Apparatur durchgeführt. Hier wurden bereits Clusterbanden von bis zu Hexameren versuchsweise zugeordnet. ${ }^{8,10}$ Eine erste Analyse der Excitonenaufspaltung wurde ebenfalls durchgeführt. ${ }^{10}$ Zur Weiterführung der Arbeiten wurden unter Nutzung der heizbaren Düse sowie des weniger auflösenden Monochromators (MC205f) zunächst Breitbandspektren aufgenommen. Hierdurch wurde die Zuordnung der Banden vereinfacht, da einerseits mittels der heizbaren Düse Spektren mit geringen Clusteranteilen und maximal Trimeren aufgenommen werden konnten und andererseits durch die gleichzeitige Erfassung des gesamten Bereichs Korrelationen zwischen verschiedenen Banden gefunden werden konnten. Danach folgte eine weitere genauso aufgebaute Spektrenreihe, die mithilfe des höher auflösenden Monochromators (MC2051) aufgenommen wurde.

Abbildungen 5.1 und 5.2 zeigen die erhaltenen Raman-Spektren von $\mathrm{H}_{2} \mathrm{O}$ und $\mathrm{D}_{2} \mathrm{O}$, wobei jeweils im oberen Teil spektral höher aufgelöste und im unteren Teil weniger aufgelöste Raman-Spektren zu sehen sind. Der Clusteranteil steigt jeweils von unten nach oben an und Spektren gleicher Farbe im unteren und oberen Teil der jeweiligen Abbildung sind bei gleichen Messbedingungen aufgenommen. Genaue Angaben über die Messbedingungen, wie z.B. Trägergas, Stagnationsdruck, Düsentemperatur und Messzeit sind in Tabelle A.3 im Anhang zu finden.

Zusätzlich zu den Raman-Spektren befinden sich jeweils im oberen, rechten Teil IRSpektren aus Ref. 21. Die Raman-Spektren, die sich in Abbildung 5.1 oben rechts befinden, sind außerdem aus Ref. 10 übernommen.

Die Bezeichnungen in den Spektren sind wie folgt aufgebaut: die Zahl steht für die jeweilige Anzahl an Monomereinheiten im Cluster, b für gebundene OH-Oszillatoren, $\mathrm{f}$ für freie Oszillatoren und a für Akzeptor. Weiterhin finden sich noch heiße Banden in den Spektren, die mit einem * gekennzeichnet sind, sowie Obertöne der Knickschwingung, die mit einem o markiert sind. Die Spektren wurden auf gleiche Höhe der QZweige der symmetrischen Streckschwingungsbande im jeweiligen pink-farbenen Spek- 
trum von 214 counts s $^{-1}\left(\mathrm{H}_{2} \mathrm{O}\right.$ oben) und 116 counts s $^{-1}\left(\mathrm{H}_{2} \mathrm{O}\right.$ unten) beziehungsweise 266 counts s $^{-1}\left(\mathrm{D}_{2} \mathrm{O}\right.$ oben) und 110 counts s$^{-1}\left(\mathrm{D}_{2} \mathrm{O}\right.$ unten) skaliert. Die Kalibrierung der wenig aufgelösten Spektren erfolgte anhand der Signale der stark Raman-aktiven gebundenen OH-Oszillatoren für Dimer, Trimer, Tetramer und Pentamer sowie der Position des Q-Zweigs der symmetrischen Streckschwingung des Monomers im höher aufgelösten Spektrum, da die Kalibrierung mittels der Neon-Linien wenig robust war.

Die $\mathrm{D}_{2} \mathrm{O}$-Spektren wurden mittels einer Achsenstreckung der Wellenzahlskala von 1.508 auf die $\mathrm{H}_{2} \mathrm{O}$-Skala angepasst und der Q-Zweig der symmetrischen Streckschwingungsbande des Monomers wurde auf der gleichen relativen Position in der Abbildung fixiert wie im $\mathrm{H}_{2} \mathrm{O}$-Spektrum. Hierdurch wird deutlich wie gut die Spektren der beiden Isotopologen korrespondieren. Diese Korrespondenz ist besonders für die gebundenen Oszillatoren der Trimere bis Pentamere erkennbar. Blauverschoben zum Q-Zweig der

\begin{tabular}{|c|c|c|c|c|c|}
\hline \multicolumn{2}{|c|}{ Wellenzahl $/ \mathrm{cm}^{-1}$} & \multirow[t]{2}{*}{ Zuordnung } & \multicolumn{2}{|c|}{ Wellenzahl $/ \mathrm{cm}^{-1}$} & \multirow[t]{2}{*}{ Zuordnung } \\
\hline $\mathrm{H}_{2} \mathrm{O}$ & $\mathrm{D}_{2} \mathrm{O}$ & & $\mathrm{H}_{2} \mathrm{O}$ & $\mathrm{D}_{2} \mathrm{O}$ & \\
\hline 3730 & 2762 & $2 \mathrm{f}$ & 3438 & 2527 & $>3 \mathrm{~b}(4 \mathrm{~b} ?)$ \\
\hline 3721 & - & $>2 \mathrm{f}$ & 3401 & 2502 & 4b(IR) \\
\hline- & 2756 & $3 \mathrm{f}$ & - & 2500 & $>3 \mathrm{~b}(5 \mathrm{~b} ?)$ \\
\hline- & 2748 & $>3 \mathrm{f}$ & 3355 & 2477 & $5 \mathrm{~b}(\mathrm{IR})$ \\
\hline 3711 & 2742 & $\gg 3 \mathrm{f}$ & 3347 & 2465 & $4 \mathrm{~b}^{*}$ \\
\hline 3657 & 2672 & 1 & 3334 & 2459 & $4 \mathrm{~b}$ \\
\hline 3651 & 2654 & $2 \mathrm{a}$ & 3310 & 2443 & $5 \mathrm{~b}$ \\
\hline 3606 & 2637 & $2 b^{*}$ & 3214 & 2375 & $>30$ \\
\hline 3602 & 2633 & $2 \mathrm{~b}$ & 3215 & - & $1 \mathrm{o}(\mathrm{IR})(212) \leftarrow(101)$ \\
\hline 3548 & - & $3 \mathrm{~b}(\mathrm{IR}-\mathrm{w})$ & 3196 & - & $1 \mathrm{o}(\mathrm{IR})(111) \leftarrow(000)$ \\
\hline 3533 & 2591 & $3 \mathrm{~b}(\mathrm{IR})$ & 3178 & - & $1 \mathrm{o}(\mathrm{IR})(110) \leftarrow(101)$ \\
\hline 3506 & 2566 & $\gg 3 \mathrm{~b}$ & 3153 & 2340 & 1 o $(\mathrm{n} 0 \mathrm{n}) \leftarrow(\mathrm{n} 0 \mathrm{n})$ \\
\hline- & 2562 & $3 b^{*}$ & 3121 & 2312 & $\gg 3 \mathrm{~b}$ \\
\hline 3491 & 2559 & $3 \mathrm{~b}$ & & & \\
\hline
\end{tabular}

Tabelle 5.1: Experimentelle Peakpositionen in $\mathrm{H}_{2} \mathrm{O}$ and $\mathrm{D}_{2} \mathrm{O}$ Expansionen sowie deren $\mathrm{Zu}-$ ordnung. Die Zahl gibt jeweils die Clustergröße an, b steht für gebundene $\mathrm{OH} \cdots \mathrm{O}$ und $\mathrm{f}$ für freie OH-Oszillatoren, a für Akzeptor, o für Oberton und * für heiße Banden. Für die Oberton-Übergänge des $\mathrm{H}_{2} \mathrm{O}$-Monomers (1o) sind außerdem die Rotationsquantenzahlen $J, K_{a}, K_{c}$ des End- und Ausgangsniveaus angegeben.

symmetrischen OH-Streckschwingung des Monomers sind neben den scharfen Peaks, die auf die komplexe Rotationsstruktur der symmetrischen und asymmetrischen Streckschwingung des Monomers zurückzuführen sind, auch breite Signale zu erkennen. Diese konnten den freien OH- bzw. OD- Oszillatoren zugeordnet werden. Es konnten Signale, die zu Dimeren (2f), Trimeren (3f), größeren (>3f) und sehr viel größeren Aggregaten $(\gg 3 \mathrm{f})$ als Trimeren gehören, identifiziert werden.

Bei den gebundenen Spezies wurde neben den schon bekannten Dimersignalen noch die symmetrische Streckschwingung des Akzeptors (2a), die bislang nur in einem He- 


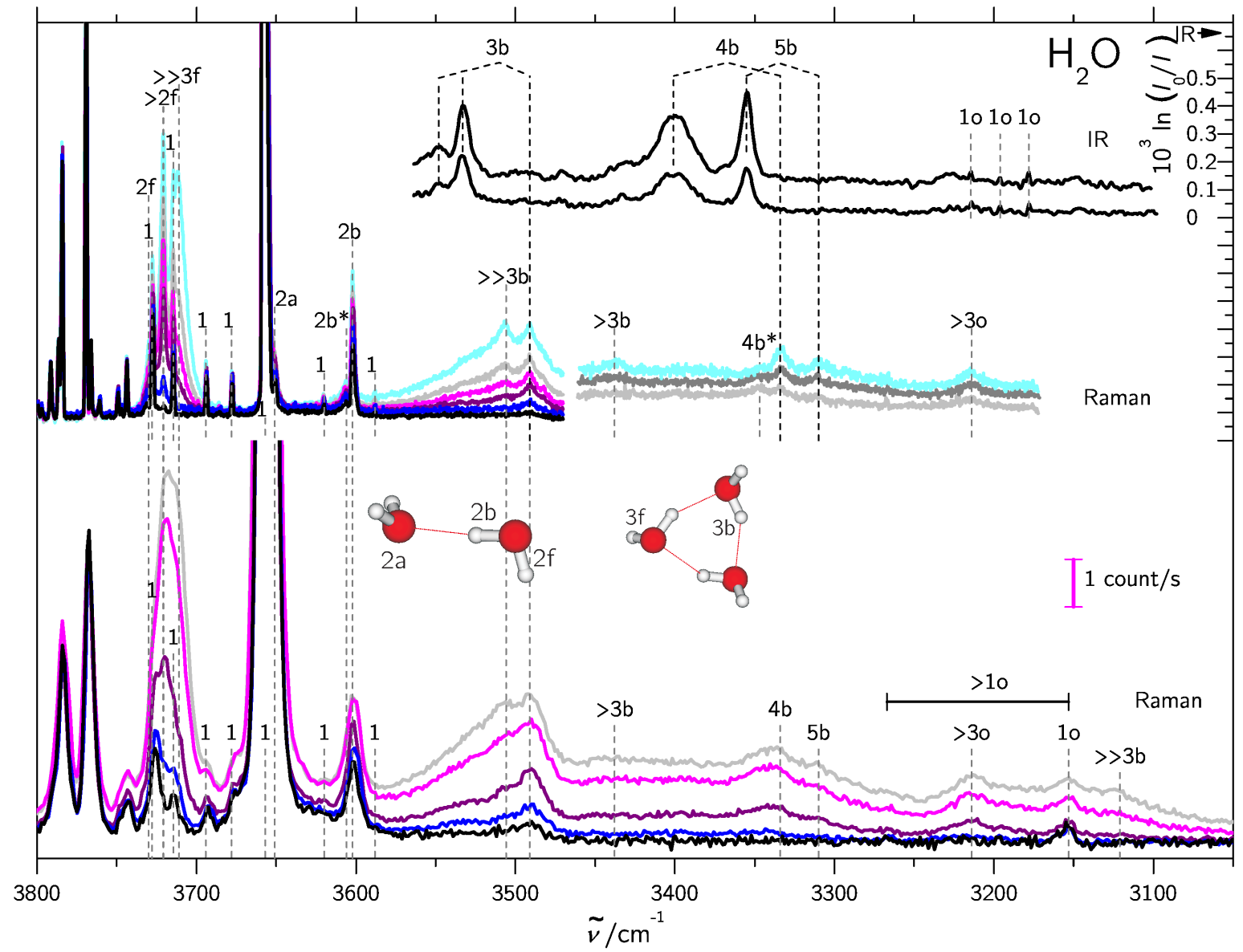

Abbildung 5.1: Raman- und IR-Spektren von Expansionen verschiedener Wasser/Trägergas-Mischungen. ${ }^{15}$ Die RamanSpektren sind auf gleiche Intensität der symmetrischen OH-Streckschwingung im jeweiligen Spektrenblock skaliert. Die Kennzeichnung der Peaks ist in Tabelle 5.1 erklärt. Angaben über Messbedingungen finden sich in Tabelle A.3 im Anhang. Der Clusteranteil in den Spektren wächst jeweils von schwarz nach hellblau (also von unten nach oben). Im oberen Bereich sind höher aufgelöste und im unteren Bereich niedriger aufgelöste Spektren gezeigt. Die IR-Spektren, die oben rechts gezeigt sind, wurden aus Ref. 21 übernommen. Die Raman-Spektren oben rechts sind außerdem aus Ref. 10 übernommen. 


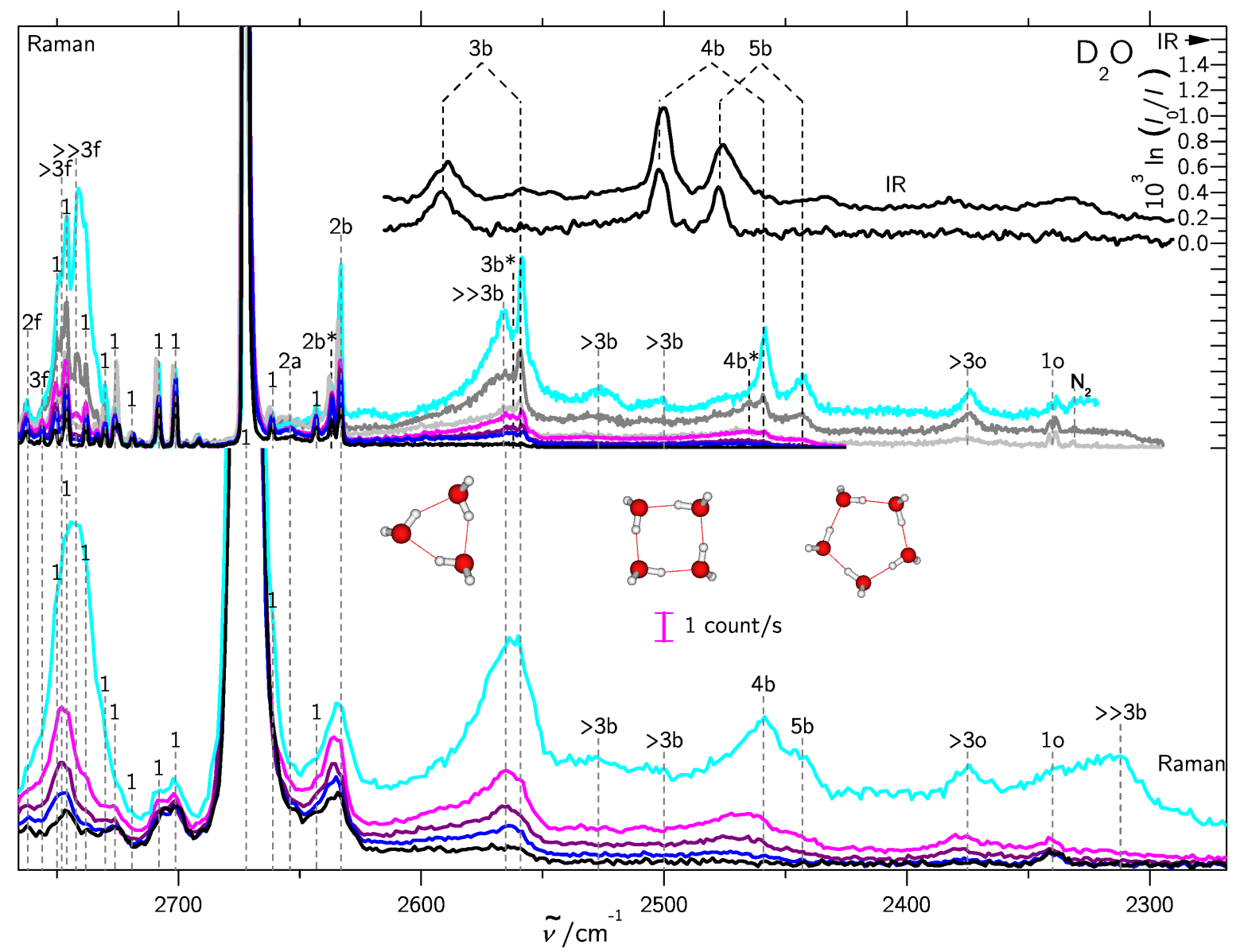

Abbildung 5.2: Wie Abbildung 5.1 aber für Expansionen von schwerem Wasser. ${ }^{15}$ Angaben über Messbedingungen finden sich in Tabelle A.3 im Anhang. Außerdem sind die Minimumsstrukturen von Trimer, Tetramer und Pentamer gezeigt. 
Nanotröpfchen-Experiment ${ }^{99}$ nachgewiesen werden konnte, ermittelt. Die Minimumsstrukturen der Trimere bis Pentamere von Wasser sind cyclisch. ${ }^{100}$ Ab dem Hexamer dominieren nicht-cyclische Strukturen. ${ }^{93,101}$ Ohne eine Größenselektionsmethode können daher nur Signale bis zum Pentamer aufgrund der systematisch größer werdenden Rotverschiebung eindeutig zugeordnet werden. Es konnten die stark Ramanaktiven Banden der Cluster identifiziert werden (s. auch Tabelle 5.1). Außerdem sind im $\mathrm{D}_{2} \mathrm{O}$-Spektrum noch schwach Raman-aktive Signale bei $2527 \mathrm{~cm}^{-1}$ (im $\mathrm{H}_{2} \mathrm{O}$-Spektrum: $3438 \mathrm{~cm}^{-1}$ ) und $2500 \mathrm{~cm}^{-1}$ zu finden. Diese wurden zunächst vorsichtig mit größer als Trimer bezeichnet $(>3 \mathrm{~b})$. Des Weiteren findet sich ein Signal blauverschoben zum Trimerpeak ( $\gg 3$ b), das erst bei sehr großen Clusterkonzentrationen auftritt. Auf eine exakte Zuordnung wurde aufgrund der fehlenden Größenselektion verzichtet. Wahrscheinlich handelt es sich aber um eine Überlagerung von Signalen der Hexamere, Heptamere und noch größerer Cluster.

Für die wärmeren Expansionen zeigen sich asymmetrische Bandenformen als generelles Merkmal, die auf thermische Anregung zurückzuführen sind. Hierin spiegelt sich die Empfindlichkeit der Streckschwingungswellenzahl auf die Stärke der Wasserstoffbrückenbindung wider, die besonders wichtig für Experimente in kondensierter Phase ${ }^{82}$ und für die Detektion von Wasserclustern in der Atmosphäre ${ }^{102,103}$ ist. Bei Verwendung von schwereren Trägergasen ergeben sich kältere Expansionen, die sowohl schmalere Banden als auch einen höheren Clusteranteil zeigen.

Wie eingangs erwähnt, liegt die größte Stärke der hier präsentierten Raman-Spektren in ihrer Komplementarität zu den bereits analysierten IR-Spektren, was besonders für cyclische Aggregate, bei denen es Schwingungen gibt, die nur sehr schwach IR- dafür aber stark Raman-aktiv sind, gilt. Durch die Kombination beider Methoden können Rückschlüsse auf die Kopplung der Oszillatoren untereinander gezogen werden. ${ }^{12}$

Im Folgenden wird die Kopplungsanalyse mittels Hückel-ähnlicher Kopplungsmatrizen zunächst für das Tetramer durchgeführt, da die Analyse hier besonders eindeutig ist. Anschließend wird als nächst-schwierigerer Fall das Trimer und abschließend das Pentamer untersucht. Es werden dabei allerdings nur die gebundenen OH-Oszillatoren betrachtet, da die freien OH-Oszillatoren aufgrund des großen Wellenzahlabstandes zu den gebundenen lediglich eine schwache Kopplung ausbilden.

\subsubsection{Tetramer}

Wie in Kapitel 2.4 beschrieben ergibt sich für das Tetramer eine Aufspaltung in ein A-symmetrisches stark Raman-aktives Niveau, als nächsthöheres ein zweifach-entartetes IR-aktives E-symmetrisches Niveau und als höchstgelegenes ein schwach Raman-aktives B-symmetrisches Niveau. Die Kopplungskonstanten $W_{1}$ und $W_{2}$ beschreiben die Kopplung zum nächsten bzw. zum übernächsten Oszillator. 
Da die Zuordnung der schwach Raman-aktiven Bande und ihre Position aufgrund des geringen Signals unsicher sind, wird als robustes Maß die Kopplungskonstante $W 4=$ $W_{1}+W_{2}$ eingeführt, die aus der Aufspaltung zwischen der stark Raman- und der stark IR-aktiven Bande bestimmt werden kann. Hieraus ergeben sich drei verschiedene Interpretationsmöglichkeiten. Bei Vernachlässigung der Kopplung zum übernächsten Nachbaroszillator $\left(W_{2}=0\right)$ ergibt sich für die Kopplungskonstante $W 4 \approx W_{1}=34 \mathrm{~cm}^{-1}$. Demgegenüber steht ein Wert von $12-13 \mathrm{~cm}^{-1}$, der aus den theoretisch vorhergesagten Wellenzahlen einer der neuesten analytischen Potentialhyperflächen ${ }^{104}$ für Wasser bestimmt wurde. Unter Berücksichtigung der Kopplungskonstanten $W_{2}$ ergeben sich Werte von $26 \mathrm{~cm}^{-1}$ für $W_{1}$ und $8 \mathrm{~cm}^{-1}$ für $W_{2}$. Diese Interpretation verbessert die Übereinstimmung zwischen Theorie und Experiment aber nicht, da für die genannte Potentialhyperfläche die Kopplung $W_{2}$ vernachlässigbar ist. Harmonische $a b$ initio Rechnungen sagen hingegen die korrekte Größenordnung der Kopplung voraus.

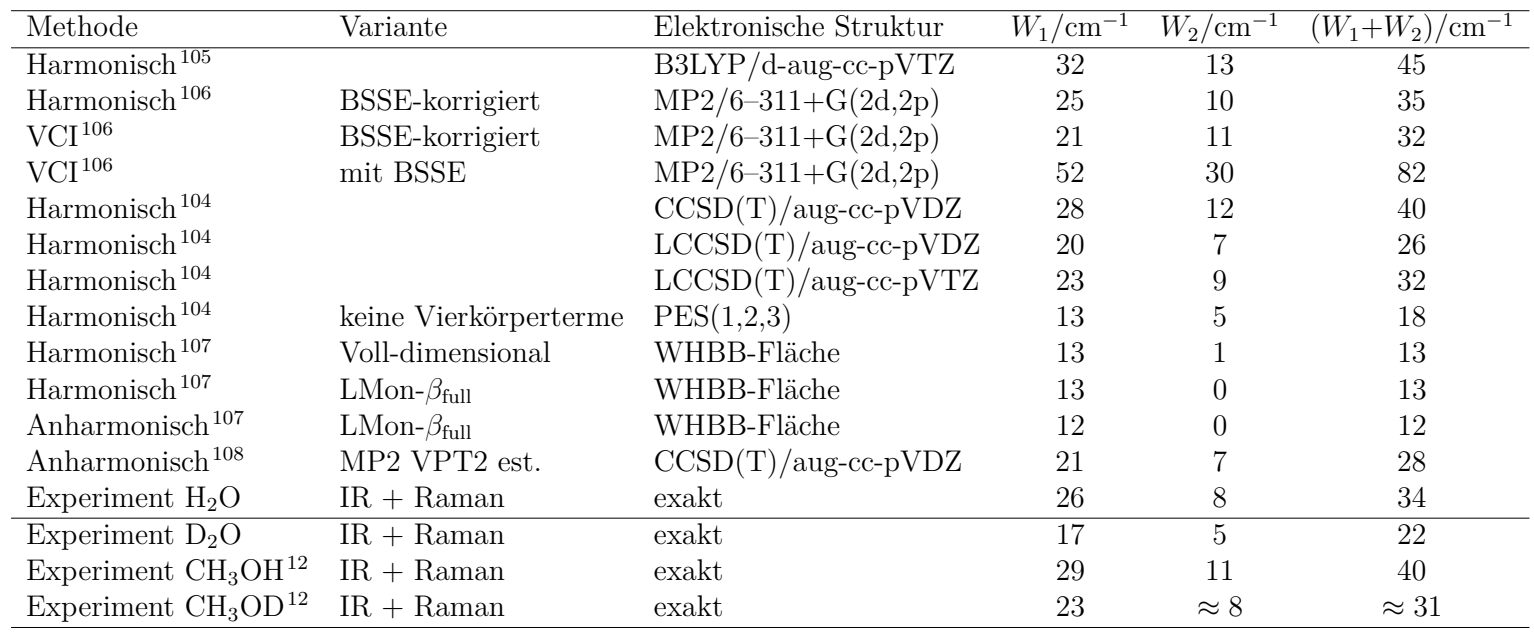

Tabelle 5.2: Verschiedene aus theoretischen Rechnungen bestimmte Werte der Kopplungskonstanten zum Nachbaroszillator $\left(W_{1}\right)$ und zum gegenüberliegenden Oszillator $\left(W_{2}\right)$ für die $\mathrm{OH}-\mathrm{Streckschwingungen} \mathrm{der} \mathrm{Wasserstoffbrücken-gebundenen} \mathrm{OH}-\mathrm{Oszillatoren} \mathrm{des} \mathrm{Was-}$ sertetramers im Vergleich mit den aus dem Experiment bestimmten Werten für $\left(\mathrm{H}_{2} \mathrm{O}\right)_{4}$ und $\left(\mathrm{D}_{2} \mathrm{O}\right)_{4}$ sowie die Werte für die entsprechenden Spezies für MeOH. ${ }^{12}$ Als besonders robuster experimenteller Wert ist außerdem die Summe $W 4=W_{1}+W_{2}$ aufgeführt.

In Tabelle 5.2 sind die experimentellen Werte der Kopplungskonstanten verschiedenen theoretischen Werten gegenüber gestellt. Außerdem sind auch die experimentellen Werte für das Methanol-Tetramer aus Ref. 12 aufgeführt. Es zeigt sich, dass die Kopplungskonstanten bei Methanol um 10-60\% größer sind als bei Wasser, was qualitativ mit einer stärkeren Wasserstoffbrückenbindung übereinstimmt. Nach Deuterierung verkleinert sich die Konstante um 30-40\% also in ähnlichem Maße wie bei Methanol. ${ }^{12}$ Die Übereinstimmung der experimentellen mit den aus harmonischen Rechnungen bestimmten Werten ist bemerkenswert gut. Während B3LYP-Rechnungen ${ }^{105}$ die Kopp- 
lung überschätzen, stimmen BSSE-korrigierte MP2 ${ }^{106}$ sowie harmonische und anharmonische CCSD(T)-Rechnungen ${ }^{104,108}$ innerhalb ihrer Fehlergrenzen mit dem Experiment überein. Hierdurch wird die Diskrepanz zu den analytischen Potentialfits noch deutlicher. Unabhängig davon, ob die Schwingungsfrequenzen innerhalb der harmonischen Näherung oder anharmonisch berechnet wurden ${ }^{104}$ und auch unabhängig von der Berücksichtigung höherer Mehrkörperterme wird die Kopplung stark unterschätzt.

\subsubsection{Trimer}

Die Kopplungen im cyclischen Trimer werden gemäß der in Kapitel 2.4 vorgestellten beiden Kopplungsfälle analysiert. Im symmetrischen Fall ergibt sich die Kopplungskonstante $W$, die hier als robuste Konstante $W 3$ verwendet werden soll und im asymmetrischen Fall ergeben sich die zwei Kopplungskonstanten $W_{1}$ und $W_{2}$. Für Wassertrimere gestaltet sich die Analyse allerdings schwieriger als für Tetramere.

In den IR-Spektren des Wasser-Trimers in verschiedenen Umgebungen ${ }^{21,89,110-112}$ zeigt sich neben einem Hauptpeak eine Nebenbande, die je nach Umgebung rot- oder blauverschoben zum Hauptpeak auftaucht. Für Deuteriumoxid verschwindet diese Nebenbande. Dieser Befund ist illustrativ in Abbildung A.6.1 im Anhang jeweils im Vergleich zum Tetramer dargestellt. Für die Kopplungsanalyse kann diese Nebenbande verschieden interpretiert werden.

Als erste Möglichkeit kann die Hauptbande als die gesuchte näherungsweise zweifach entartete IR-Bande interpretiert und die Nebenbande ignoriert werden $\left(3_{A}\right)$. Als zweite Möglichkeit können beide Banden, obwohl sie stark unterschiedliche Intensitäten aufweisen als die beiden Komponenten der nahezu entarteten IR-aktiven Schwingung aufgefasst werden $\left(3_{B}\right)$. Hierdurch ergeben sich Kopplungskonstanten für den symmetrischen Fall von $W=14 \mathrm{~cm}^{-1}$ und $17 \mathrm{~cm}^{-1}$. Der wahrscheinlichste Wert liegt dazwischen, da als plausibelster Referenzpunkt der Schwerpunkt der beiden Banden verwendet werden sollte. Für Ne-Matrixspektren ${ }^{90}$ liegen die Werte bei $19 \mathrm{~cm}^{-1}\left(3_{A}\right)$ und $17 \mathrm{~cm}^{-1}$ $\left(3_{B}\right)$, wobei sich der relativ große Wert für die Variante $3_{A}$ daraus ergibt, dass die Schulter der IR-Bande hier rotverschoben auftaucht, ansonsten ergibt sich eine gute Übereinstimmung.

In Tabelle 5.3 sind die experimentellen Kopplungskonstanten für den symmetrischen $(W)$ und asymmetrischen Kopplungsfall $\left(W_{1}, W_{2}\right)$ denen verschiedener theoretischer Modellrechnungen sowie den Kopplungen in Deuteriumoxid und Methanol gegenüber gestellt. Zur Berechnung der symmetrischen Kopplungskonstante wurde für die theoretischen Werte jeweils Schema $3_{B}$ gewählt und der Mittelwert der beiden (gleich intensiven) stark IR-aktiven Banden als Referenzpunkt verwendet. Trotz der großen Unsicherheit in der Zuordnung der IR-Banden zeigt die Kopplungskonstante $W$ in Analogie zum Tetramer das erwartete Verhalten für Deuterierung und Methylierung. 


\begin{tabular}{|c|c|c|c|c|c|}
\hline Methode & Variante & Elektronische Struktur & $W / \mathrm{cm}^{-1}$ & $W_{1} / \mathrm{cm}^{-1}$ & $W_{2} / \mathrm{cm}^{-1}$ \\
\hline Harmonisch $^{106}$ & BSSE-korrigiert & MP2/6-311+G(2d,2p) & 17 & 19 & 13 \\
\hline $\mathrm{VCI}^{106}$ & BSSE-korrigiert & MP2/6-311+G(2d,2p) & 19 & 21 & 16 \\
\hline $\mathrm{VCI}^{106}$ & mit BSSE & $\mathrm{MP} 2 / 6-311+\mathrm{G}(2 \mathrm{~d}, 2 \mathrm{p})$ & 24 & 26 & 20 \\
\hline Harmonisch $^{104}$ & & $\operatorname{PES}(1,2,3)$ & 11 & 13 & 7 \\
\hline Harmonisch $^{104}$ & & CCSD(T)/aug-cc-pVTZ & 20 & 22 & 16 \\
\hline Harmonisch $^{104}$ & & CP-CCSD(T)/aug-cc-pVTZ & 18 & 20 & 14 \\
\hline Harmonisch $^{104}$ & & LCCSD(T)/aug-cc-pVTZ & 15 & 16 & 11 \\
\hline Harmonisch $^{109}$ & & LCCSD(T)/aug-cc-pVQZ & 21 & 23 & 17 \\
\hline Harmonisch ${ }^{109}$ & $3 \mathrm{~b} 5$ fit & WHBB-Fläche & 8 & 10 & 4 \\
\hline Harmonisch $^{109}$ & $3 \mathrm{~b} 6$ fit & WHBB-Fläche & 15 & 17 & 10 \\
\hline Harmonisch ${ }^{108}$ & & $\operatorname{CCSD}(\mathrm{T}) /$ aug-cc-pVDZ & 18 & 20 & 15 \\
\hline Anharmonisch ${ }^{108}$ & MP2 VPT2 est. & CCSD(T)/aug-cc-pVDZ & 15 & 17 & 9 \\
\hline Experiment $\mathrm{H}_{2} \mathrm{O}\left(3_{A}\right)$ & IR+Raman & exakt & 14 & 14 & 14 \\
\hline Experiment $\mathrm{H}_{2} \mathrm{O}\left(3_{B}\right)$ & IR+Raman & exakt & 17 & 20 & 9 \\
\hline Experiment $\mathrm{D}_{2} \mathrm{O}$ & IR+Raman & exakt & 11 & 11 & 11 \\
\hline Experiment $\mathrm{CH}_{3} \mathrm{OH}^{12}$ & IR+Raman & exakt & 20 & 21 & 17 \\
\hline Experiment $\mathrm{CH}_{3} \mathrm{OD}^{12}$ & IR+Raman & exakt & 13 & 14 & 11 \\
\hline
\end{tabular}

Tabelle 5.3: Verschiedene theoretische Werte der Kopplungskonstanten $W 3=W$ für ein $C_{3}$-symmetrisches Trimer (einfaches Hückel-Modell mit einem A-symmetrischen (Ramanaktiven) Niveau und den zweifach entarteten E-symmetrischen Niveaus (IR-aktiv)) sowie die Kopplungskonstanten $W_{1}$ und $W_{2}$ für den komplexeren Fall, bei dem die Wasserstoffbrückenbindungen unterschiedlich stark sind (asymmetrische Kopplung). Die experimentellen Kopplungskonstanten für das Trimer sind weniger zuverlässig bestimmbar als die des Tetramers aufgrund der geringeren Symmetrie, der möglicherweise großen Tunnelaufspaltung und der unterschiedlichen Intensitäten der zwei IR-Banden.

Der Vergleich mit den theoretischen Werten ergibt erneut ein sehr robustes Bild für harmonische Rechnungen, da die Werte relativ unabhängig vom Rechenniveau sind. Das zeigt sich auch für eine Variante des WHBB-Potentialfits, nämlich der Variante 3b6 zur Beschreibung der 3-Körper Beiträge. ${ }^{109}$ In der zweiten Variante 3b5 ergibt sich hingegen eine halb so große Kopplungskonstante. Das legt nahe, dass diese Parametrisierung für die Tetramer-Rechnungen ${ }^{98,113}$ verwendet wurde, was möglicherweise $\mathrm{zu}$ den im vorherigen Kapitel gezeigten Diskrepanzen beiträgt.

\subsubsection{Pentamer}

Für das Pentamer wird die in Kapitel 2.4 vorgestellte Kopplungsmatrix für $C_{5 h^{-} \text {-symme- }}$ trische Aggregate verwendet. Auch hier werden Kopplungskonstanten für die Kopplung zum ersten $\left(W_{1}\right)$ und zweiten Nachbaroszillator $\left(W_{2}\right)$ defininiert. Die Aufpaltung der Energieniveaus erfolgt in ein $\mathrm{A}^{\prime}$-symmetrisches Raman-aktives Level, ein zweifach entartetes IR-aktives $\mathrm{E}_{1}^{\prime}$ und ein schwach Raman-aktives, zweifach entartetes $\mathrm{E}_{2}^{\prime}$-Niveau.

Allerdings weist die Minimumsstruktur des Pentamers keine Symmetrie $\left(C_{1}\right)$ auf, ${ }^{38}$ 
sondern es handelt sich um einen Ring, bei dem vier O-Atome näherungsweise in einer Ebene liegen und das fünfte aus der Ebene heraus steht. Die Umwandlungsbarriere der zwei spektroskopisch nicht unterscheidbaren Strukturen, bei denen sich ein Sauerstoffatom einmal ober- und einmal unterhalb der Ebene befindet, ist jedoch sehr klein, ${ }^{114}$ so dass für die im Jet vorhandenen Temperaturen und aufgrund der raschen Tunnelbewegung der freien $\mathrm{H}$-Atome bereits eine effektive $C_{5 h}$-Symmetrie angenommen werden kann.

Für die Berechnung der Kopplungskonstanten aus den theoretischen Wellenzahlen werden jeweils die Mittelwerte der zwei höchstliegenden gebundenen OH-Streckschwingungen, die beide schwach Raman-aktiv sind sowie die Mittelwerte der zwei stark IRaktiven Banden der gebundenen OH-Oszillatoren verwendet. Da auch hier die Bandenposition der schwach Raman-aktiven Bande unsicher ist, wird als robuste Kopplungskonstante zudem eine Linearkombination der Kopplungskonstanten für die Kopplung zum Nachbar- und zum darauffolgenden Oszillator verwendet, die im symmetrisierten Modell nicht von dieser Bande abhängig ist.

Sie ist definiert als $W 5=W_{1}+\frac{\sqrt{5}+1}{\sqrt{5}-1} \cdot W_{2}$.

Es zeigt sich, dass die Pentamerbanden im Vergleich zu denen von Tetramer und Trimer trotz einer geringeren Ringspannung und der größeren Kooperativität der Wasserstoffbrückenbindungen enger zusammen liegen. Eine Zuordnung der schwach Ramanaktiven Bande ist nur im $\mathrm{D}_{2} \mathrm{O}$-Spektrum möglich. Wenn die Bande bei $2500 \mathrm{~cm}^{-1}(>3 \mathrm{~b})$ als die gesuchte dritte Bande interpretiert wird, ergeben sich Kopplungskonstanten von $W_{1}=14 \mathrm{~cm}^{-1}$ und $W_{2}=4 \mathrm{~cm}^{-1}$, die im Vergleich zu den entsprechenden Werten des Tetramers von 17 und $5 \mathrm{~cm}^{-1}$ also kleiner sind.

Ein ähnlicher Befund ergibt sich beim Vergleich der robusten Kopplungskonstanten $W 5$ mit den aus theoretischen Rechnungen bestimmten Werten (vgl. Tab. 5.4). Der experimentelle Wert ist systematisch kleiner als die theoretischen selbst für Rechenniveaus, die sich für die Tetramer- und Trimer-Kopplungskonstanten als gut geeignet erwiesen.

Möglicherweise ist dieser Sachverhalt auf einen anharmonischen Effekt zurückzuführen. Zur Klärung der Fragestellung wird der Spektralbereich um $3200 \mathrm{~cm}^{-1}$ (bzw. $2400 \mathrm{~cm}^{-1}$ für $\mathrm{D}_{2} \mathrm{O}$ ) hinzugezogen. ${ }^{115}$ Hier befinden sich die ersten Obertöne der Knickschwingungsbanden. Es zeigt sich, dass blauverschoben zum Monomerpeak (10) ein weiteres Signal zu finden ist, welches aufgrund seiner Entwicklung mit steigendem Clusteranteil Tetrameren und Pentameren zuzuordnen ist. Dies lässt sich besonders in Abbildung 5.3 nachvollziehen, wo Clustersignale, die dieselbe Größe wie die >3o Bande besitzen, im Differenzspektrum wegfallen. Ein zum Monomer blauverschobener ${ }^{116}$ Oberton der Knickschwingung wurde bereits für das Trimer in Ne-Matrizen bei $3190 \mathrm{~cm}^{-1}$ gefunden. ${ }^{90}$ 


\begin{tabular}{lllccc}
\hline Methode & Variante & Elektronische Struktur & $W_{1} / \mathrm{cm}^{-1}$ & $W_{2} / \mathrm{cm}^{-1}$ & $W 5=W_{1}+\frac{\sqrt{5}+1}{\sqrt{5}-1} \cdot W_{2} / \mathrm{cm}^{-1}$ \\
\hline Harmonisch $^{105}$ & skaliert & B3LYP/d-aug-cc-pVDZ & 34 & 10 & 60 \\
Harmonisch $^{106}$ & BSSE-korrigiert & MP2/6-311+G(2d,2p) & 26 & 7 & 45 \\
VCI $^{106}$ & BSSE-korrigiert & MP2/6-311+G(2d,2p) & 26 & 6 & 42 \\
VCI $^{106}$ & mit BSSE & MP2/6-311+G(2d,2p) & 43 & 34 & 133 \\
Harmonisch $^{107}$ & Voll-dimensional & WHBB & 14 & 0 & 13 \\
Harmonisch $^{107}$ & LMon- $\beta_{\text {dimer }}$ & WHBB & 14 & 0 & 14 \\
Anharmonisch $^{107}$ & LMon- $\beta_{\text {dimer }}$ & WHBB & 15 & 0 & 14 \\
Harmonisch $^{108}$ & & CCSD(T)/aug-cc-pVDZ & 30 & 9 & 53 \\
Anharmonisch $^{108}$ & MP2 VPT2 est. & CCSD(T)/aug-cc-pVDZ & 36 & 21 & 90 \\
Experiment $\mathrm{H}_{2} \mathrm{O}$ & IR + Raman & exakt & - & - & 33 \\
\hline Harmonisch & 38 & $\mathrm{D}_{2} \mathrm{O}$ \\
Experiment $\mathrm{D}_{2} \mathrm{O}$ & $\mathrm{IR}+$ Raman & MP2/aug-cc-pVDZ & 24 & 8 & 44 \\
\hline
\end{tabular}

Tabelle 5.4: Verschiedene theoretische Werte für die Kopplungskonstante zum Nachbar$\left(W_{1}\right)$ und zum zweiten Nachbaroszillator $\left(W_{2}\right)$ für das Modell eines $C_{5 h^{-} \text {-symmetrischen }}$ Wasserpentamers. Da die Minimumsstruktur des Pentamers keine Symmetrie und somit keine Entartungen aufweist, wurden die Mittelwerte der IR-aktiven Banden, die dem $\mathrm{E}_{1^{-}}^{\prime}$ Niveau entsprechen, sowie die Mittelwerte der zwei höchstfrequenten Raman-aktiven Banden, die dem $\mathrm{E}_{2}^{\prime}$-Niveau entsprechen, zur Bestimmung der Kopplungskonstanten verwendet. Die Linearkombination $W 5=W_{1}+\frac{\sqrt{5}+1}{\sqrt{5}-1} \cdot W_{2}$ in der letzten Spalte ist unabhängig von der experimentell schwierig zuzuordnenden schwach Raman-aktiven Bande $\left(\mathrm{E}_{2}^{\prime}\right)$ und somit ein sehr robustes Maß für die Kopplung.

In den vorliegenden Spektren ist die Raman-Intensität des Signals im Vergleich zum Monomeroberton ungewöhnlich groß für einen Clusteroberton. Im Regelfall sollte die relative Intensität der Cluster aufgrund des großen Monomeranteils in der Expansion wesentlich kleiner sein. Ein Zusammenfallen der Obertöne von Tetramer und Pentamer ist außerdem im ungestörten System eher unwahrscheinlich. ${ }^{105}$

Eine Erklärung kann eine mit zunehmender Clustergröße stärker werdende FermiResonanz liefern. Die Abstände zwischen dem höchstliegenden ersten Oberton der Knickschwingung und der Raman-aktiven Streckschwingung werden mit zunehmender Clustergröße immer kleiner, so dass die Resonanz besonders stark für das Pentamer ist. Die Folge ist, dass die Intensität des Obertons erhöht wird, während seine Frequenz erniedrigt wird. Außerdem wird die Raman-aktive 5b Bande zu höheren Wellenzahlen verschoben. Diese Vermutung kann durch eine Modellrechnung mit berechneten Raman-Frequenzen und Depolarisationsverhältnissen ${ }^{105}$ bestätigt werden. In Tabelle 5.5 ist diese Modellrechnung angegeben. Eine Fermi-Resonanz ist am wahrscheinlichsten bei einem geringen Depolarisationsverhältnis, wie es für die niederfrequenteste Bande der OH-Streckschwingungsfundamentalen der Fall ist, und wenn der Wellenzahlabstand $(\Delta(\mathrm{b}-\mathrm{o}))$ besonders klein ist. Daraus folgt, dass die Resonanz für das Pentamer am stärksten ist, was die ungewöhnlich kleine Kopplungskonstante (W5) erklärt. Die Größenordnung der Fermi-Resonanzkopplung ${ }^{117}$ kann auf $20-50 \mathrm{~cm}^{-1}$ für das $\mathrm{H}_{2} \mathrm{O}$-Pentamer abgeschätzt werden. 


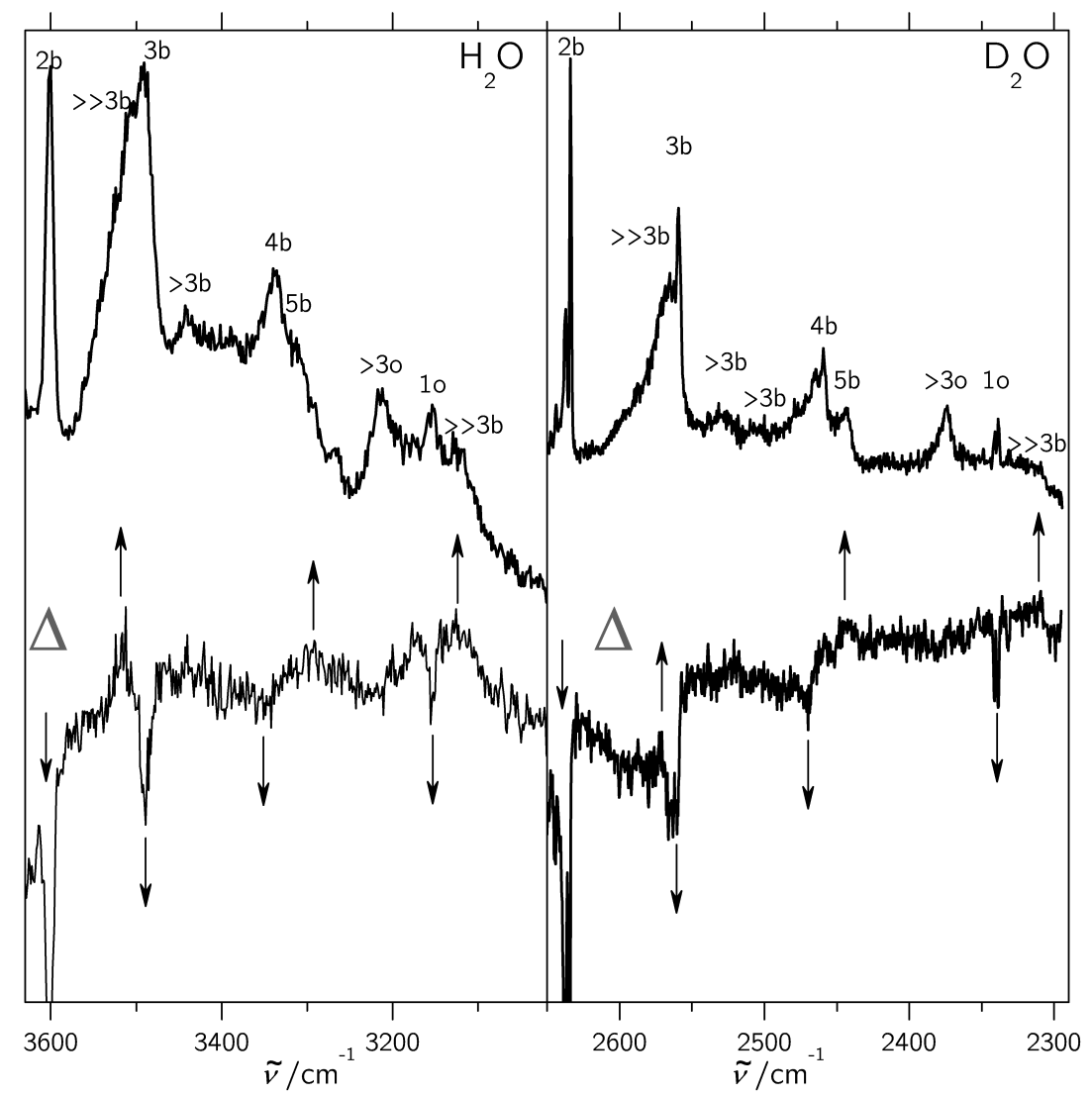

Abbildung 5.3: Differenzspektren $\Delta$ (unten), die zeigen, dass die Intensität der $>3$ o Bande (obere Spektren) in Jetexpansionen von $\mathrm{H}_{2} \mathrm{O}$ (links) und $\mathrm{D}_{2} \mathrm{O}$ (rechts) hauptsächlich auf Tetramer- und Pentamerbeiträge zurückzuführen ist. ${ }^{15}$ Außerdem zeigt sich, dass Banden, die mit $\gg 3$ b bezeichnet sind von Hexameren und größeren Clustern stammen. Vor Subtraktion der beiden Spektren, die einen unterschiedlichen relativen Clusteranteil haben, wurden diese auf die $>3$ o Bande skaliert. Im Differenzspektrum ergeben sich somit negative Banden für Clustergrößen kleiner als $>3$ o und positive Peaks für Cluster, die größer als $>3$ o sind. 


\begin{tabular}{c|ccc}
\hline $\begin{array}{c}\text { Cluster- } \\
\text { größe }\end{array}$ & \multicolumn{3}{|c}{ B3LYP/d-aug-cc-pVDZ $\omega_{n}^{105}$} \\
\hline 1 & 1622 & 0.68 & 504 \\
3 & 1658 & 0.39 & 266 \\
& 1634 & 0.64 & 314 \\
& 1631 & 0.75 & 320 \\
4 & 1681 & 0.25 & 63 \\
& 1651 & 0.75 & 123 \\
& 1636 & 0.75 & 153 \\
5 & 1689 & 0.17 & 23 \\
& 1681 & 0.05 & 39 \\
& 1660 & 0.74 & 81 \\
& 1652 & 0.62 & 97 \\
& 1640 & 0.73 & 121 \\
\hline
\end{tabular}

Tabelle 5.5: Berechnete Knickschwingungswellenzahlen $\omega_{n}^{\text {bend }}$ der Ring-Cluster und Raman Depolarisationsverhältnisse $\rho$ wurden verwendet um den Abstand

$\Delta(\mathrm{b}-\mathrm{o})=\nu(n \mathrm{~b})-\left(\nu(1 \mathrm{o})+2\left(\omega_{n}^{\text {bend }}-\omega_{1}^{\text {bend }}\right)\right)$ zwischen der niederfrequentesten $\mathrm{OH}-$ Streckschwingungsfundamentalen des gebundenen $\mathrm{OH} n \mathrm{~b}$ und den ersten Obertönen der Knickschwingung abzuschätzen. Hierdurch kann qualitativ gezeigt werden, dass eine FermiResonanz für das Pentamer am wahrscheinlichsten ist.

Für die korrekte Berechnung von Wasserclustern ist die Einbeziehung von anharmonischen Effekten unerlässlich. Von den zahlreichen theoretischen Modellen beinhalten allerdings nur wenige anharmonische Effekte ${ }^{118}$ und Raman-Intensitäten. ${ }^{105}$ Die beschriebenen Resultate sollen einen Anreiz schaffen die bestehenden Modelle hinsichtlich der Kopplung zwischen den Oszillatoren weiter zu entwickeln.

\subsubsection{Weitere Clusterbanden}

Weitere bislang noch nicht zugeordnete Peaks finden sich um 3100 und $3500 \mathrm{~cm}^{-1}$ $(\gg 3 \mathrm{~b})$. Diese könnten aufgrund ihrer relativen Intensitäten auf Hexamere und größere Cluster zurückgeführt werden (siehe Abb. 5.3). Wegen der fehlenden Größenselektion ${ }^{119}$ im Experiment ist eine eindeutige Zuordnung jedoch nicht möglich. Für die Struktur eines möglichen Hexamers kann zunächst die cyclische Struktur durch Vergleich mit Ergebnissen aus He-Nanotröpfchen-Eperimenten ${ }^{110}$ ausgeschlossen werden und auch das Buch-Isomer ist eher unwahrscheinlich, was sich beim Vergleich mit der Theorie ${ }^{118}$ zeigt. Somit kommen die Käfig- und die Prismastrukturen ${ }^{93}$ infrage, die beide in den genannten Bereichen Schwingungsbanden zeigen. 


\subsection{OH-Knickschwingungen und $\mathrm{OH}$-Streckschwingungsobertöne}

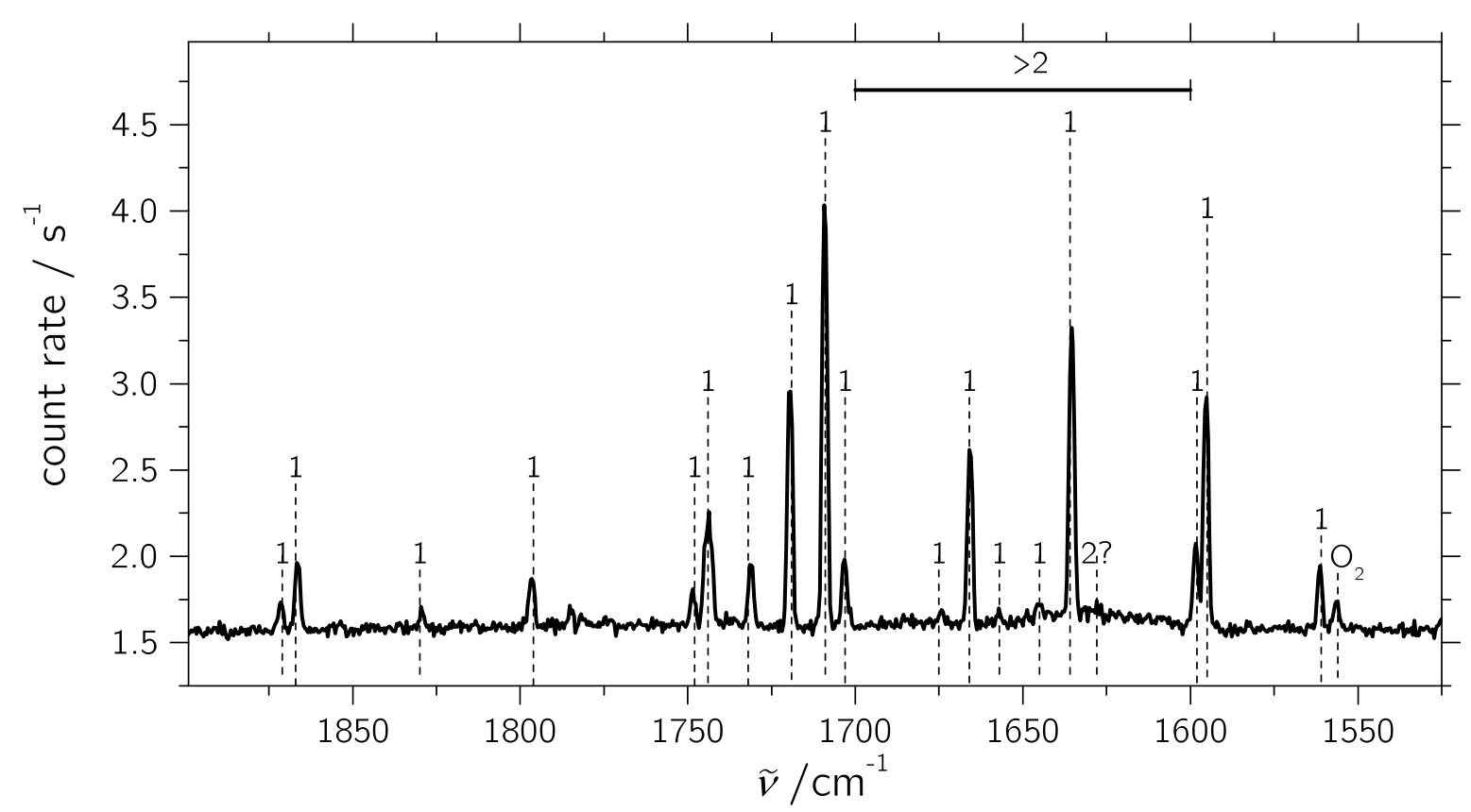

Abbildung 5.4: Raman-Jetspektrum von $\mathrm{H}_{2} \mathrm{O}$ im OH-Knickschwingungsbereich. Das Spektrum ist dominiert von den scharfen Monomerpeaks. Eine schwache Bande bei $1628 \mathrm{~cm}^{-1}$ kann aber in Übereinstimmung mit Ref. 116 und 120 dem Dimer zugeordnet werden. Außerdem ist eine breite Absorption im Bereich zwischen 1600 und $1700 \mathrm{~cm}^{-1}$ zu finden, die auf größere Cluster zurückzuführen ist. Eine genaue Zuordnung ist jedoch nicht möglich. Das Spektrum weist eine geringe Verunreinigung mit Luftsauerstoff auf. Details zu den Messbedingungen finden sich im Anhang A.3.

Auch im Bereich der OH-Knickschwingung wurden mithilfe des höher auflösenden Monochromators Raman-Spektren aufgenommen. Ein exemplarisches Spektrum ist in Abbildung 5.4 gezeigt. Der OH-Knickschwingungsbereich ist durch die scharfen Peaks der Monomer-Rotationsschwingungsübergänge gekennzeichnet. Die Zuordnung der Monomerpeaks erfolgte durch Vergleich mit Ref. 121. Die Peakpositionen sind in Tabelle A.6.1 im Anhang inklusive der Angabe der Rotationsquantenzahlen $\left(J, K_{a}, K_{c}\right)$ des Monomers von Ausgangs- und Endniveau angegeben. Für die Cluster konnte in Übereinstimmung mit Ref. 116 und 120 eine schwach Raman-aktive Dimerbande bei $1628 \mathrm{~cm}^{-1}$ zugeordnet werden, wobei es sich hier aufgrund des sehr schwachen Signals um eine vorläufige Zuordnung handelt. Des Weiteren zeigt das Spektrum in Abbildung 5.4 eine breite Absorptionsbande im Bereich 1600-1700 $\mathrm{cm}^{-1}$. Laut Ref. 116 und 122 sind hier die Knickschwingungsbanden größerer Cluster zu finden. Eine genaue Zuordnung ist aufgrund der Breite der Bande allerdings nicht möglich. Neben 
dem OH-Knickschwingungsbereich wurde auch der OD-Knickschwingungsbereich der Jetexpansionen gemessen, allerdings ist hier aufgrund der enger zusammen liegenden Banden eine noch geringere Chance einer Zuordnung zu Clusterbanden vorhanden, weswegen auf eine Darstellung verzichtet wurde.

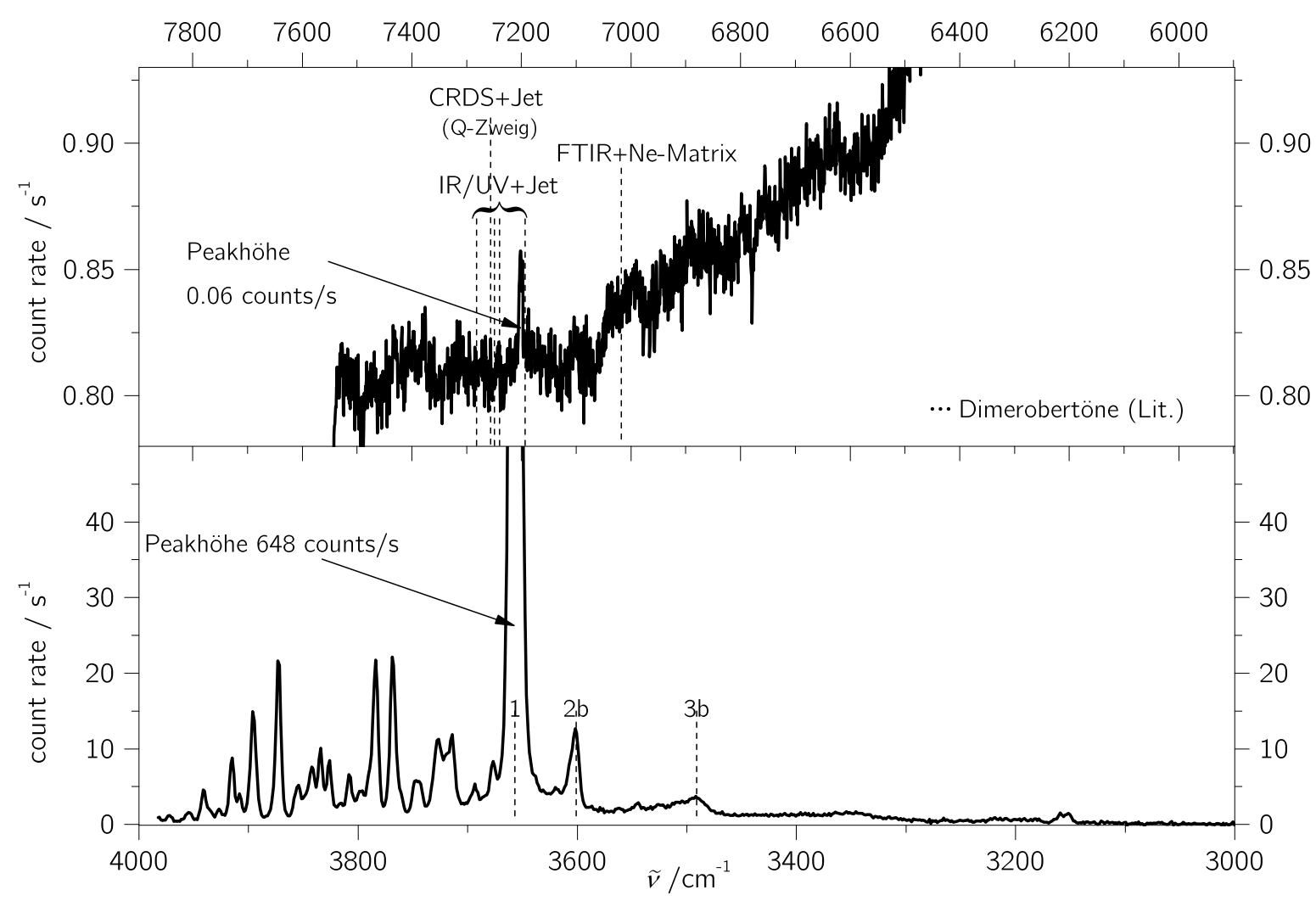

Abbildung 5.5: Oberton- (oben) und Fundamentalspektrum (unten) der OHStreckschwingungsbanden von Wasser. mittels vertikaler Linien sind die bereits aus der Literatur bekannten Dimerobertöne ${ }^{123-125}$ im oberen Spektrum eingezeichnet. Details zu den Messbedingungen finden sich im Anhang A.3.

Ein weiterer interessanter Bereich des Schwingungsspektrums der Wassercluster findet sich um $7000 \mathrm{~cm}^{-1}$. Hier sind die ersten Obertöne der OH-Streckschwingungsbanden zu finden. Diese sollten genau wie die Fundamentalen rotverschoben zum Monomerpeak auftauchen, wobei hier die Zuordnung aufgrund der großen Anzahl an Möglichkeiten an Obertönen und Kombinationsbanden komplizierter ist. ${ }^{124}$ Zur Aufnahme der Spektren wurde der niedriger auflösende Monochromator MC205f verwendet, der in diesem Spektralbereich ca. $1100 \mathrm{~cm}^{-1}$ umfasst. Erste Messungen bei dem für die $\mathrm{OH}-\mathrm{Knick}$ - und $\mathrm{OH}-\mathrm{Streckschwingungsfundamentalen} \mathrm{verwendeten} \mathrm{Düsenabstand}$ von $2 \mathrm{~mm}$ zeigten keine Signale. Auch bei $1 \mathrm{~mm}$ Abstand konnten keine Peaks identifiziert werden. Nach Verringerung des Abstandes auf $0.5 \mathrm{~mm}$ und einer Gesamtmesszeit von 2.5 Stunden konnte das in Abbildung 5.5 (oben) gezeigte Spektrum erhalten wer- 
den. In der Abbildung ist das Obertonspektrum im Vergleich zu einem Spektrum der OH-Streckschwingungsfundamentalen bei gleichem Düsenabstand gezeigt. Die Wellenzahlbereiche sind so gewählt, dass der Bereich der Obertöne genau doppelt so groß ist wie der der Fundamentalen und die Bandenzentren der Monomere die gleiche relative Position besitzen. Auf diese Weise ist die Veränderung der diagonalen Anharmonizität bei der Dimerbildung direkt im Spektrum erkennbar. ${ }^{126,127} \mathrm{Im}$ Raman-Spektrum können jedoch lediglich der bereits aus der Literatur bekannte Oberton des Monomers bei einer Wellenzahl von $7202 \mathrm{~cm}^{-1}$ und die zugehörige diagonale Anharmonizitätskonstante von $x_{11}=-56 \mathrm{~cm}^{-1}$ bestimmt werden. ${ }^{123,128}$ Das Intensitätsverhältnis des Obertons zur Fundamentalen ist bereits für das Monomer 1:11000, wobei hier als grober Richtwert das Verhältnis der Peakhöhen verwendet wurde. Dementsprechend ist eine Aufnahme der Obertöne der Cluster bei den gegebenen Messbedingungen nicht möglich. In Ne-Matrixspektren wurde der IR-Oberton des gebundenen OH-Oszillators des Dimer-Donors bei $7018 \mathrm{~cm}^{-1}$ gefunden. ${ }^{123}$ Weitere Werte für Dimerobertöne wurden in einem IR/UV-Doppelresonanzexperiment in der Jetexpansion im Bereich um $7200 \mathrm{~cm}^{-1}$ ermittelt ${ }^{124}$ und kürzlich in einem CRDS-Experiment bestätigt. ${ }^{125}$ Einige der Literatur-bekannten Dimerbanden sind in Abbildung 5.5 (oben) mittels gestrichelter vertikaler Linien gekennzeichnet. Das gezeigte Raman-Spektrum weist zwar breite, stark verrauschte Strukturen bei 7000 und $6900 \mathrm{~cm}^{-1}$ auf, eine Zuordnung ist aber aufgrund des schlechten S/N-Verhältnisses nicht möglich. Es handelt sich hier höchstwahrscheinlich um Artefakte. Eine erneute Messung mit zusätzlicher Aufnahme eines Hintergrundspektrums, wie sie im Bereich der Fundamentalen bereits erfolgt ist, könnte Abhilfe schaffen. Eine starke Verbesserung des Signal-zu-Rauschverhältnisses könnte außerdem durch die Verwendung von kavitätsverstärkter Raman-Spektroskopie erzielt werden.

\subsection{Zusammenfassung und Ausblick}

Durch Aufnahme von Raman-Spektren kleiner Wassercluster in der Gasphase konnten unter Nutzung von IR-Daten ${ }^{21}$ Kopplungskonstanten für die Kopplung der gebundenen OH-Streckschwingungen bestimmt werden. In Tabelle 5.6 sind die experimentell bestimmten robusten Kopplungskonstanten inklusive einer Fehlerabschätzung (s. Tabellenunterschrift) für Trimer, Tetramer und Pentamer noch einmal zusammengefasst. Diese liefern Einblicke in die Energieübertragung zwischen lokalen OH-Streckoszillatoren. Die Entwicklungen der gefundenen Kopplungskonstanten mit Deuterierung oder Methylierung sind systematisch und erwartungsgemäß und ergeben somit ein konsistentes Bild. Die Abhängigkeit der Konstanten von der Ringgröße ist gering, was sich durch die in Kapitel 5.2.3 erläuterte Fermi-Resonanz mit dem ersten Oberton der Knickschwingung erklären lässt. Diese wird vom Trimer zum Pentamer immer stärker, wodurch sie der durch die größere Kooperativität stärker werdenden 


\begin{tabular}{cccc}
\hline & $W 3 / \mathrm{cm}^{-1}$ & $W 4 / \mathrm{cm}^{-1}$ & $W 5 / \mathrm{cm}^{-1}$ \\
\hline $\mathrm{H}_{2} \mathrm{O}$ & $14-17$ & 34 & 33 \\
$\mathrm{D}_{2} \mathrm{O}$ & 11 & 22 & 25 \\
$\mathrm{CH}_{3} \mathrm{OH}^{12}$ & 20 & 40 & \\
\hline
\end{tabular}

Tabelle 5.6: Robuste experimentell bestimmte Kopplungskonstanten für die gebundenen OH-Streckschwingungen für Trimere $(W 3)$, Tetramere $(W 4)$ und Pentamere $(W 5)$ von Wasser, Deuteriumoxid und Methanol. Die Fehler der Konstanten können aus dem Fehler der Raman-Bandenposition und der Bandenbreite auf $<2 \mathrm{~cm}^{-1}$ für $W 4$ und $<3 \mathrm{~cm}^{-1}$ für $W 5$ abgeschätzt werden.

Wasserstoffbrückenbindungen und somit der zu erwartenden stärkeren Kopplung entgegen wirkt.

Die experimentellen Ergebnisse liefern Richtwerte für die Weiterentwicklung der theoretischen Methoden und außerdem einen Anreiz die bisherigen Potentiale, die die IRFrequenzen zwar gut voraussagen jedoch bei den Raman-Frequenzen bislang versagen, hinsichtlich dieses Aspekts weiterzuentwickeln.

Im Sinne einer Interpretation der Aufspaltung als zeitabhängiger Energiefluss zwischen lokalen Oszillatoren ${ }^{12,102}$ kann eine Zeitkonstante für den Energieabfluss von 0.3 ps für das Trimer abgeschätzt werden. ${ }^{129,130}$ Für Pentamer und Tetramer sind die Werte noch geringer. In der vernetzten Flüssigkeit ist dieser Energiefluss irreversibel, so dass es zur Energiedissipation kommt. Die vorliegenden Ergebnisse liefern eine qualitative Erklärung für die ultraschnelle Energieumverteilung in der Flüssigkeit, die in fs-Experimenten nachgewiesen wurden, ${ }^{85,131,132}$ ohne dass Fermi-Resonanzen und andere Relaxationskanäle in Betracht gezogen werden müssen. Die letzteren Mechanismen kommen besonders für Cluster, die größer als das Trimer sind, hinzu und führen dazu, dass die Energie letztendlich in niederfrequente Schwingungen umverteilt wird. ${ }^{133}$ Der erste Schritt der Energieumverteilung in benachbarte lokale Oszillatoren kann jedoch zumindest bis zum Tetramer in harmonischer Näherung relativ gut erklärt werden, was sich in der guten Übereinstimmung der theoretischen mit den experimentellen Kopplungskonstanten widerspiegelt.

Bei der Entwicklung von Modellpotentialen für Wasser zur Simulation der fs-Dynamik $^{92,98,109}$ ist es somit äußerst wichtig, diese auf ihre Fähigkeit die IR/Raman-Aufspaltung korrekt vorherzusagen zu testen. Die Wasserstoffbrücken-induzierte Excitonenaufspaltung ist zwar ein sehr subtiler Effekt, zeigt sich aber als ein wichtiges Merkmal der Clusterpotentialhyperflächen.

Studien des OH-Knickschwingungsbereichs und des Obertonbereichs der OH-Streckschwingungen ergaben bislang keine eindeutigen Ergebnisse. Besonders die Studie der Obertöne wäre interessant fortzuführen, da hier bislang nur wenig Literatur über RamanSpektren in der Gasphase bekannt ist. 


\section{Systeme zur Untersuchung von Isomerisierungen}

Eine mögliche Auswirkung der IR-Laseranregung ist das Induzieren von Isomerisierungen. Ein geeignetes System zur Untersuchung dieser Isomerisierungsreaktionen ist 3,3,3-Trifluorpropanol. Im folgenden Kapitel werden Vorexperimente zur Ermittlung des Energieunterschiedes zwischen den beiden im Jet stabilen Konformeren vorgestellt. Zunächst wird anhand des Testsystems 2,2,2-Trifluorethanol eine experimentelle Nullpunktskorrektur etabliert. Anschließend folgt die Analyse von 3,3,3-Trifluorpropanol und abschließend wird die experimentelle Ermittlung von Energieunterschieden auf $N$ Methylformamid angewendet, das eine sehr hohe Umwandlungsbarriere besitzt.

\section{$6.12,2,2-$ Trifluorethanol}

2,2,2-Trifluorethanol (TFE) ist ein sehr gut spektroskopisch in Gasphase, Flüssigkeit, Festkörper und Edelgasmatrizen untersuchtes Molekül. ${ }^{134-139}$ Auch in Jetexpansionen wurden bereits Monomere und Aggregate von TFE mittels FTIR- und Raman-Spektroskopie analysiert. ${ }^{8,60,140,141}$ In der vorliegenden Arbeit soll das System, das in der Jetexpansion als gauche-Konformer vorliegt, ${ }^{141}$ als Testsystem dienen. Durch Zuordnung aller Fundamentalbanden kann die quantenchemisch berechnete Nullpunktsenergie mit experimentellen Werten korrigiert und so die Größenordnung dieser Korrektur bestimmt werden. Diese Methode kann für Systeme, bei denen bspw. zwei Monomerkonformere im Jet vorliegen, zur Abschätzung des Energieunterschiedes zwischen den Konformeren genutzt werden. ${ }^{18}$ Hierzu wird ein einfaches Morse-Oszillator-Modell angenommen. Außerdem wird davon ausgegangen, dass die verwendeten quantenchemischen Rechnungen die harmonischen Frequenzen korrekt voraussagen und die Summe der außerdiagonalen Anharmonizitätsterme vernachlässigbar klein ist. Der Nullpunktsenergiebeitrag $E_{0, i}$ einer Schwingung $i$ kann dann folgendermaßen aus den harmonisch berechneten Wellenzahlen $\omega_{i}$ und den experimentell beobachteten (anharmonischen) Wellenzahlen $\widetilde{\nu_{i}}$ bestimmt werden: ${ }^{18}$

$$
\frac{E_{0, i}}{h c N_{\mathrm{A}}}=\frac{1}{8} \cdot\left(3 \omega_{i}+\widetilde{\nu_{i}}\right)
$$




\begin{tabular}{|c|c|c|c|}
\hline Raman-Jet & IR-Jet ${ }^{60}$ & Raman-GP ${ }^{135}$ & Zuordnung \\
\hline 3657 & 3657 & 3659 & $\nu_{\mathrm{OH}}$ \\
\hline 3556 & 3555 & & Dimer-Donor \\
\hline 3002 & 3002 & 3003 & $\nu_{\mathrm{CH}}^{a}$ \\
\hline 2962 & 2963 & & Dimer/Cluster \\
\hline 2948 & 2949 & 2951 & $\nu_{\mathrm{CH}}^{s}\left(\mathrm{FR}\right.$ mit $\left.2 \delta_{\mathrm{CH}_{2}}\right)$ \\
\hline 2918 & & & Cluster? \\
\hline 2897 & 2897 & 2900 & $2 \delta_{\mathrm{CH}_{2}}\left(\mathrm{FR}\right.$ mit $\left.\nu_{\mathrm{CH}}^{s}\right)$ \\
\hline 1566 & & & ? (überlagert mit $\mathrm{O}_{2}$ ) \\
\hline 1458 & 1457 & 1450 & $\delta_{\mathrm{CH}_{2}}$ \\
\hline & 1415 & & $\omega_{\mathrm{CH}_{2}}$ \\
\hline 1366 & 1369 & 1370 & $t_{\mathrm{CH}_{2}} / \delta_{\mathrm{OH}}$ \\
\hline 1293 & 1292 & 1292 & $\nu_{\mathrm{CF}_{3}}^{a}$ \\
\hline 1264 & 1264 & & $\nu_{\mathrm{CF}_{3}}^{a} / \delta_{\mathrm{OH}}$ \\
\hline 1183 & 1184 & & $\nu_{\mathrm{CF}_{3}}^{s}$ \\
\hline 1142 & 1142 & 1142 & $t_{\mathrm{CH}_{2}} / \delta_{\mathrm{OH}}$ \\
\hline 1110 & & & Cluster \\
\hline 1088 & 1089 & 1090 & $\nu_{\mathrm{CO}}$ \\
\hline 940 & 940 & 944 & $\gamma_{\mathrm{CH}_{2}}$ \\
\hline 835 & 831 & 828 & $\nu_{\mathrm{CC}}\left(\mathrm{FR}\right.$ mit $\left.2 \delta_{\mathrm{CCO}} / \tau_{\mathrm{OH}}\right)$ \\
\hline 828 & & & $2 \delta_{\mathrm{CCO}} / \tau_{\mathrm{OH}}\left(\mathrm{FR}\right.$ mit $\left.\nu_{\mathrm{CC}}\right)$ \\
\hline 665 & & 666 & $\delta_{\mathrm{CF}_{3}}^{s}$ \\
\hline 547 & & 548 & $\delta_{\mathrm{CF}_{3}}^{a}$ \\
\hline 541 & & & $\delta_{\mathrm{CF}_{3}}^{a}$ \\
\hline 416 & & 416 & $\delta_{\mathrm{CCO}} / \tau_{\mathrm{OH}}$ \\
\hline 364 & & 358 & $\gamma_{\mathrm{CF}_{3}} / \tau_{\mathrm{OH}}$ \\
\hline 283 & & & $\tau_{\mathrm{OH}}$ \\
\hline 235 & & 236 & $2 \tau_{\mathrm{CF}_{3}}\left(\mathrm{FR}\right.$ mit $\left.2 \delta_{\mathrm{CCO}}\right)$ \\
\hline 218 & & & $\delta_{\mathrm{CCO}}\left(\mathrm{FR}\right.$ mit $\left.2 \tau_{\mathrm{CF}_{3}}\right)$ \\
\hline 116 & & 106 & $\tau_{\mathrm{CF}_{3}}$ \\
\hline
\end{tabular}

Tabelle 6.1: Im Raman-Jetexperiment bestimmte Peakpositionen in $\mathrm{cm}^{-1} \mathrm{im}$ Vergleich mit IR-Jet-Daten ${ }^{60}$ und Raman-Gasphasendaten. ${ }^{135}$ Die Zuordnung wurde in Übereinstimmung mit Ref. 60 und mittels harmonischer Rechnungen getroffen. Es konnten drei FermiResonanzen (FR) identifiziert werden. Die Bezeichnungen sind wie folgt aufgebaut: $\nu$ für Streck-, $\delta$ für Knick-, $\gamma$ für Schaukel-, $t$ für „Twist“-, $\omega$ für Wackel- und $\tau$ für Torsionsschwingungen. Die Superskripte $s$ und $a$ bezeichnen symmetrische und asymmetrische Schwingungen. 


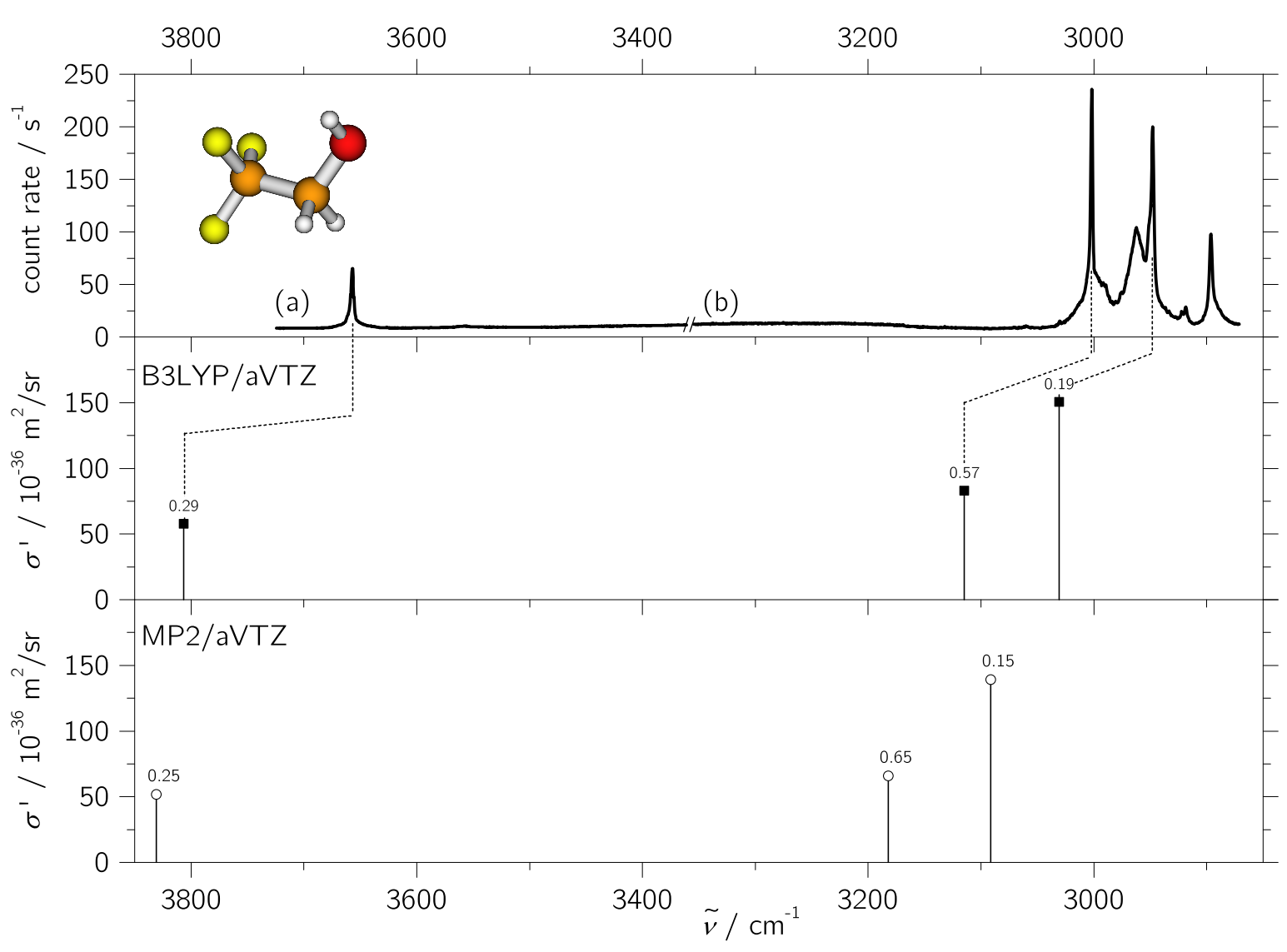

Abbildung 6.1: Raman-Spektren von 2,2,2-Trifluorethanol im OH- (a) und CHStreckschwingungsbereich (b) im Vergleich zu harmonischen B3LYP- und MP2Rechnungen. Für die berechneten Spektren sind auch die Depolarisationsverhältnisse angegeben mit $\rho^{\prime}=0.86$ für eine vollständig depolarisierte Schwingung. Details zu den Messbedingungen sind im Anhang A.3 aufgeführt. Oben links ist außerdem die Minimumsstruktur des 2,2,2-Trifluorethanolmonomers (berechnet auf B3LYP/aVTZNiveau) gezeigt.

Für diese Abschätzung sind genaue Bandenpositionen erforderlich. Aufgrund der thermischen Verbreiterung sind Gasphasenspektren bei Raumtemperatur daher eher ungeeignet. Die IR-Jetspektren ${ }^{60,141}$ umfassen nur den Bereich von $3700-800 \mathrm{~cm}^{-1}$ au- $^{-}$ ßerdem können möglicherweise Positionen einzelner Banden bspw. bei schwacher IRAktivität oder sehr breiter Signatur nicht gut bestimmt werden. In der vorliegenden Arbeit wurde der spektrale Bereich mittels Raman-Spektroskopie um die niederfrequenten Banden bis zu einer Wellenzahl von $50 \mathrm{~cm}^{-1}$ erweitert.

In Abbildungen 6.1 bis 6.3 sind die erhaltenen Raman-Jetspektren im Vergleich zu quantenchemischen Rechnungen gezeigt und in Tabelle 6.1 sind die erhaltenen Bandenpositionen denen aus FTIR-Jetmessungen ${ }^{60}$ und Raman-Gasphasenmessungen ${ }^{135}$ 
gegenübergestellt. Die Zuordnung erfolgte in Übereinstimmung mit Ref. 60 sowie unter Zuhilfenahme von quantenchemischen Rechnungen, wobei auch die Depolarisationsverhältnisse $\rho^{\prime}$ genutzt wurden.

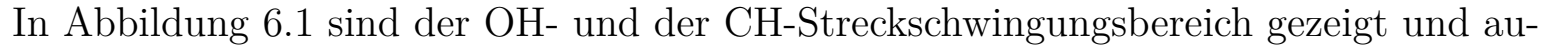
ßerdem ist oben links das gauche-Konformer von TFE dargestellt, das das globale Minimum ist. In diesem Spektralbereich konnten die in Ref. 8 und 60 gefundenen Werte reproduziert werden. Die Fermiresonanz, die zwischen dem ersten Oberton einer $\mathrm{CH}_{2^{-}}$ Deformationsschwingung bei $2897 \mathrm{~cm}^{-1}$ und der symmetrischen CH-Streckschwingung bei $2949 \mathrm{~cm}^{-1}$ auftritt, wurde in Ref. 60 bereits gefunden, die Kopplung jedoch nicht weiter analysiert, da der Fokus der genannten Arbeit auf den TFE-Aggregaten lag. Neben der Dimerakzeptorbande bei $3633 \mathrm{~cm}^{-1}$ und der Dimerdonorbande bei $3554 \mathrm{~cm}^{-1}$ wurden in Ref. 60 im OH-Streckschwingungsbereich auch Hinweise auf Trimere und Tetramere gefunden. Im vorliegenden Spektrum ist abweichend von den vorherigen IR-Ergebnissen im CH-Streckschwingungsbereich noch ein Signal bei $2918 \mathrm{~cm}^{-1} \mathrm{zu}$ erkennen, das vermutlich von TFE-Clustern stammt. Aufgrund der fehlenden Konzentrationsreihe, die hier wegen der bereits guten Datenlage für die Monomerbanden unterlassen wurde, kann aber keine sichere Zuordnung getroffen werden.

In Abbildung 6.2 ist der Bereich zwischen 1650 und $800 \mathrm{~cm}^{-1}$ gezeigt. Spektrenpaar (a) zeigt den Bereich zwischen 1400 und $800 \mathrm{~cm}^{-1}$, wobei in schwarz ein Spektrum mit senkrecht zur Streuebene polarisiertem Laser und in grau das zugehörige Spektrum mit parallel polarisiertem Laser gezeigt ist. Spektrenpaar (b) ist in einem etwas anderen Spektralbereich aufgenommen. Beide Spektren (schwarz und hellgrau) sind mit senkrecht zur Streuebene polarisiertem Laser allerdings bei verschiedenen Konzentrationen aufgenommen. Sie wurden auf die gleiche Monomerkonzentration wie Spektrum (a, schwarz) skaliert.

Bis auf eine Bande bei $1415 \mathrm{~cm}^{-1}$, die eine sehr schwache Raman-Aktivität aufweist, konnten alle im FTIR-Jetspektrum gefundenen Banden reproduziert wurden. Zusätzlich wurde eine Bande bei $1566 \mathrm{~cm}^{-1}$ gefunden, die allerdings mit einer Verunreinigung durch Luftsauerstoff überlagert ist. Die Bande ist jedoch zu breit um ausschließlich auf Sauerstoff zurückgeführt zu werden. Eine Clusterbande ist aber aufgrund der nicht systematischen Entwicklung mit steigender Konzentration auszuschließen. Bei der CHKnickschwingungsbande bei $1458 \mathrm{~cm}^{-1}$ fällt auf, dass die quantenchemischen Rechnungen eine nahezu vollständig depolarisierte Bande vorhersagen, während die experimentelle Bandenform auf eine polarisierte Bande hindeutet. Die experimentellen Spektren zeigen, dass die Bande mit steigender Konzentration überproportional anwächst, was auf eine Überlagerung mit Clustersignalen hindeutet. Es kann sich allerdings auch um einen thermischen Effekt handeln, denn die Bandenposition verschiebt sich bei höherer Konzentration zu niedrigerer Wellenzahl und somit in Richtung des Gasphasenwertes aus Ref. 135. Bei den weiteren Banden stimmen die berechneten und experimentellen Depolarisationsverhältnisse qualitativ gut überein. 


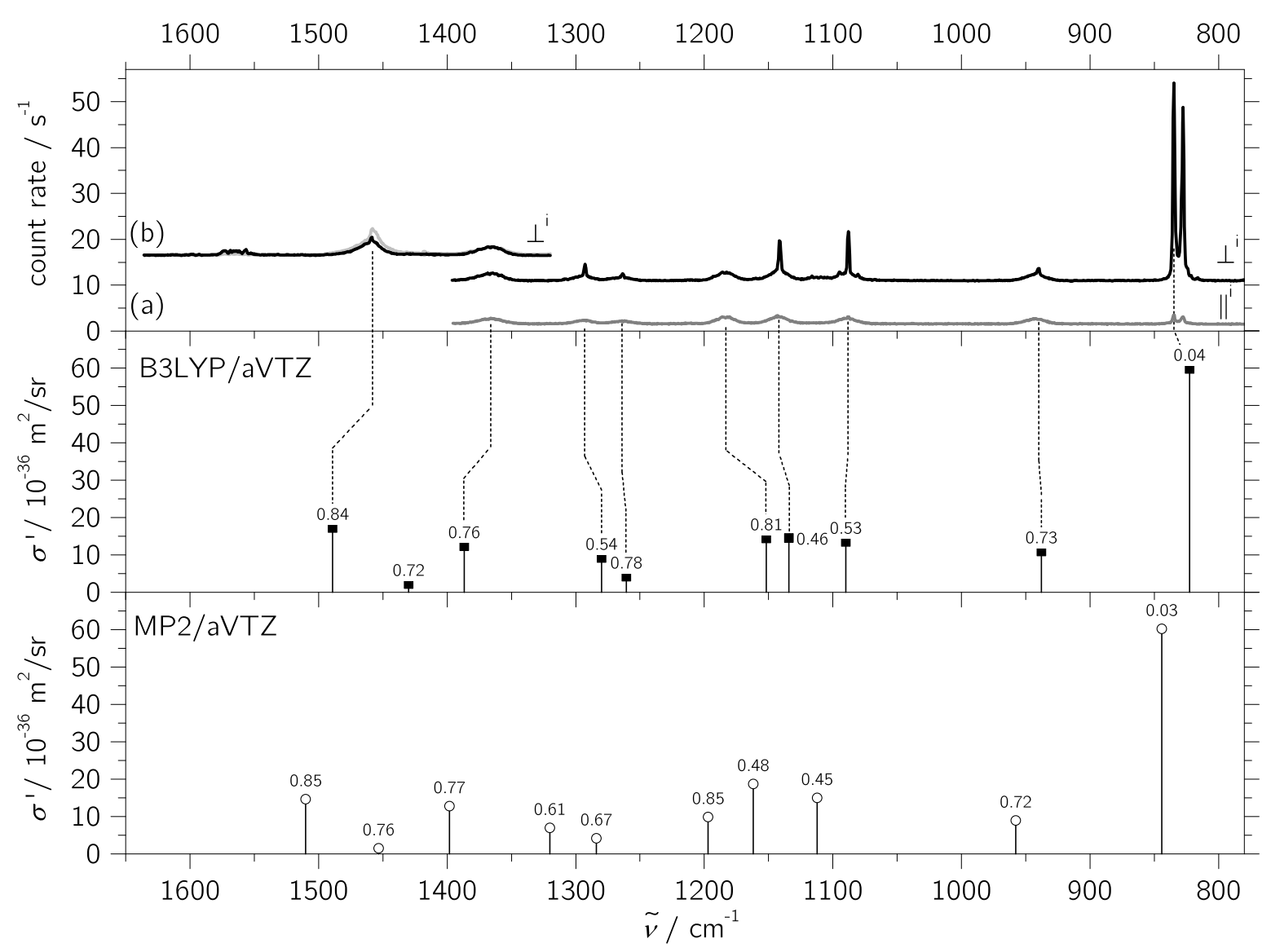

Abbildung 6.2: Raman-Spektren von 2,2,2-Trifluorethanol im Bereich $1650-800 \mathrm{~cm}^{-1} \mathrm{im}$ Vergleich zu harmonischen B3LYP- und MP2-Rechnungen (auch hier inklusive der Angabe der Depolarisationsverhältnisse). (a) Spektren, die bei senkrecht $\left(\perp^{i}\right)$ und parallel $\left(\|^{i}\right)$ zur Streuebene polarisiertem Laser aufgenommen wurden. Es tritt eine Fermiresonanz zwischen den Banden bei 835 und $828 \mathrm{~cm}^{-1}$ auf. (b) Spektren, die bei senkrecht $\left(\perp^{i}\right)$ zur Streuebene polarisiertem Laser aufgenommen wurden, einen etwas anderen Wellenzahlbereich abdecken und bei höherer Konzentration gemessen wurden. Diese wurden auf die Monomerkonzentration von (a, schwarz) skaliert. (b, schwarz): skaliert mit einem Faktor von 0.5; (b, hellgrau): bei höherer Konzentration aufgenommenes Raman-Jetspektrum, skaliert mit einem Faktor von 0.18. Es zeigt sich, dass die Bande bei $1458 \mathrm{~cm}^{-1}$ mit steigender Konzentration wächst, was auf eine Überlagerung mit Clusterbanden schließen lässt. Die Verschiebung der Bandenposition zu niedrigeren Wellenzahlen kann aber auch auf einen thermischen Effekt hindeuten. Details zu den Messbedingungen sind im Anhang A.3 aufgeführt. 
Ein weiteres auffälliges Signal ist die Dublettstruktur bei $830 \mathrm{~cm}^{-1}$. Diese wurde bereits in Ref. 136 gefunden, hier jedoch auf das gauche- und das trans-Konformer zurückgeführt. Aufgrund der niedrigen Temperaturen und des großen Energieunterschiedes von ca. $8 \mathrm{~kJ} / \mathrm{mol}$ relativ zum gauche-Konformer kann eine signifikante Population des trans-Konformers im Jet ausgeschlossen werden. ${ }^{141}$ Eine sehr viel wahrscheinlichere Interpretation der zwei Banden ist daher eine Fermiresonanz zwischen dem ersten Oberton einer CCO-Knickschwingung ${ }^{\mathrm{a}}$ und der Fundamentalen der CC-Streckschwingung. Diese erklärt auch die vergleichbare Intensität der beiden Banden.

In Abbildung 6.3 ist der niederfrequente Bereich gezeigt mit gleicher Farbcodierung wie in Abbildung 6.2 (a). Die Depolarisationsverhältnisse aus Rechnungen und Experiment stimmen auch hier qualitativ gut überein und sichern somit die Zuordnung ab. Beim Vergleich mit den Raman-Gasphasenmessungen ${ }^{135}$ ergeben sich Unterschiede in der Bandenposition von bis $\mathrm{zu} 10 \mathrm{~cm}^{-1}$ ( $\mathrm{CF}_{3}$-Torsion). Außerdem konnte eine Bande bei $541 \mathrm{~cm}^{-1}$ identifiziert werden, die aufgrund ihrer Breite und der Überlagerung mit der Bande bei $547 \mathrm{~cm}^{-1}$ in Ref. 135 nicht gefunden wurde.

Des Weiteren konnte eine Fermiresonanz zwischen dem ersten Oberton der $\mathrm{CF}_{3}$-Torsion bei $235 \mathrm{~cm}^{-1}$ und einer CCO-Deformationsschwingung bei $218 \mathrm{~cm}^{-1}$ bestimmt werden, die für die große Intensität des Obertons verantwortlich ist.

Es wurden drei Bandenpaare identifiziert, bei denen eine Fermiresonanz zwischen den Schwingungen auftritt. Über Gleichung 6.2 können unter der Annahme verschwindender Streuintensität des ungestörten Obertons aus der beobachteten Aufspaltung $\Delta E$ und dem Intensitätsverhältnis $R$ die entstörte Aufspaltung $\Delta E_{0}$ und hieraus die entstörten Bandenpositionen berechnet werden. ${ }^{117}$

$$
\frac{\Delta E_{0}}{\Delta E}=\frac{R-1}{R+1}
$$

\begin{tabular}{|c|c|c|c|c|}
\hline$\widetilde{\nu_{i}} / \mathrm{cm}^{-1}$ & $\Delta E / \mathrm{cm}^{-1}$ & $R$ & $\Delta E_{0} / \mathrm{cm}^{-1}$ & ${\widetilde{\nu_{i}}}^{\text {entstört }} / \mathrm{cm}^{-1}$ \\
\hline 2948 & \multirow{2}{*}{51} & \multirow{2}{*}{2} & \multirow{2}{*}{17} & 2937 \\
\hline 2897 & & & & 2921 \\
\hline 835 & \multirow{2}{*}{7} & \multirow{2}{*}{1.1} & \multirow{2}{*}{2} & 833 \\
\hline 828 & & & & 831 \\
\hline 235 & \multirow{2}{*}{17} & \multirow{2}{*}{2} & \multirow{2}{*}{6} & 227 \\
\hline 218 & & & & 221 \\
\hline
\end{tabular}

Tabelle 6.2: Experimentelle $\left(\widetilde{\nu_{i}}\right)$ und entstörte Bandenpositionen $\left(\widetilde{\nu}_{i}^{\text {entstört }}\right)$ und Aufspaltungen $\left(\Delta E, \Delta E_{0}\right)$ sowie das Intensitätsverhältnis $R$ der in den Raman-Jetspektren identifizierten durch Fermiresonanzen gekoppelten Moden.

${ }^{a}$ In den harmonischen Rechnungen zeigt sich, dass für diese Schwingungskoordinate neben der CCO-Knickbewegung auch eine OH-Torsion auftritt. Daher die in Tabelle 6.1 angegebene Zuordnung. 


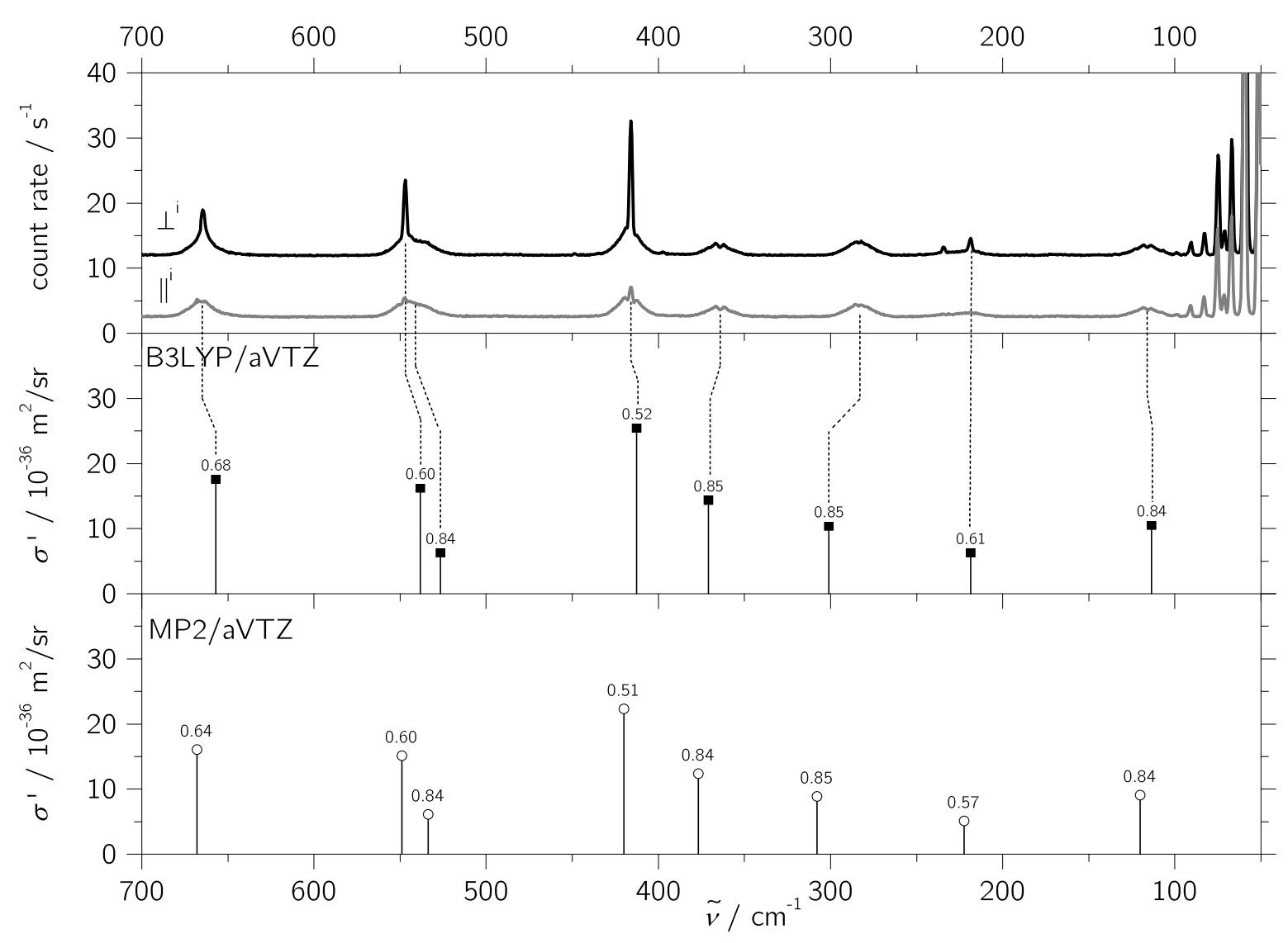

Abbildung 6.3: Raman-Jetspektren von 2,2,2-Trifluorethanol im Bereich $700-50 \mathrm{~cm}^{-1} \mathrm{im}$ Vergleich zu harmonischen B3LYP- und MP2-Rechnungen (auch hier inklusive der Angabe der Depolarisationsverhältnisse) einmal mit senkrecht $\left(\perp^{i}\right)$ und einmal parallel $\left(\|^{i}\right)$ zu Streuebene polarisiertem Anregungslaser. Es zeigt sich, dass bei ca. $545 \mathrm{~cm}^{-1}$ zwei Banden überlagert sind. Des Weiteren tritt eine Fermi-Resonanz zwischen den Banden bei 235 und $218 \mathrm{~cm}^{-1}$ auf. Details zu den Messbedingungen sind im Anhang A.3 aufgeführt. 


\begin{tabular}{lccccc}
\hline & B3LYP/aVTZ & \multicolumn{2}{c}{ B2PLYP-D3BJ/ } & \multicolumn{2}{c}{ MP2/aVTZ } \\
& def2-TZVP & & \\
& (harm.) & (harm.) & (VPT2) & (harm.) & (VPT2) \\
\hline ZPE $^{\text {calc }} / \mathrm{kJ} \mathrm{mol}^{-1}$ & 150.0 & 151.9 & 150.1 & 152.8 & 151.0 \\
$\mathrm{ZPE}^{\exp } / \mathrm{kJ} \mathrm{mol}^{-1}$ & 149.5 & 150.9 & - & 151.6 & - \\
\hline
\end{tabular}

Tabelle 6.3: Nullpunktsenergien aus harmonischen und anharmonischen (VPT2) quantenchemischen Rechnungen $\mathrm{ZPE}^{\text {calc }}$ sowie die nach Gleichung 6.1 experimentell korrigierten Nullpunktenergien ZPE ${ }^{\exp }$.

Die Ergebnisse sind in Tabelle 6.2 gezeigt, wobei die Intensitätsverhältnisse grob geschätzt wurden. Die entstörten Bandenpositionen können dann in die experimentell korrigierte Nullpunktsenergie mit einbezogen werden.

Gemäß Gleichung 6.1 können nach erfolgter Zuordnung die Nullpunktsenergiebeiträge experimentell korrigiert werden. Die entsprechenden berechneten Wellenzahlen und die experimentell korrigierten Werte befinden sich in Tabelle A.7.1 im Anhang. Die erhaltenen Nullpunktsenergien (ZPE ${ }^{\exp }$ ) sind in Tabelle 6.3 den unkorrigierten Werten $\left(\mathrm{ZPE}^{\text {calc }}\right)$ gegenüber gestellt.

Je nach quantenchemischer Rechenmethode verringert sich die Nullpunktsenergie durch die experimentelle Korrektur um $0.5-1.2 \mathrm{~kJ} / \mathrm{mol}$. Auffällig ist hierbei, dass für die B3LYP-Rechnung eine signifikante Anzahl an Banden (8 von 21) existiert, deren harmonische Wellenzahlwerte kleiner als die experimentell bestimmten sind. Diese sind in Tabelle A.7.1 im Anhang mit einem * markiert. Dies lässt darauf schließen, dass die harmonischen Wellenzahlen bereits zu niedrig und somit auch die Nullpunktsenergie zu klein vorausgesagt wird, was durch die experimentelle Korrektur nicht signifikant verbessert werden kann. Für die B2PLYP- und MP2-Rechnung ergibt sich nur je eine Bande mit abweichendem Verhalten. Für alle anderen Schwingungen werden die harmonischen Werte größer als die experimentellen vorhergesagt, so dass hier die Annahme einer korrekten Vorhersage der harmonischen Werte eher gerechtfertigt ist. Der Effekt der experimentellen Korrektur beläuft sich daher mindestens auf $1-1.2 \mathrm{~kJ} / \mathrm{mol}$. Der Vergleich mit den anharmonischen Rechnungen zeigt, dass der Effekt für beide Rechenmethoden in einer ähnlichen Größe von $1.8 \mathrm{~kJ} / \mathrm{mol}$ vorhergesagt wird.

Der Vergleich der anharmonisch berechneten Wellenzahlwerte mit den experimentell bestimmten in Tabelle A.7.1 zeigt für B2PLYP besonders im OH- und CH-Streckschwingungsbereich Abweichungen. Außerdem wird die Reihenfolge zweier Fermiresonanzpaare, nämlich der $\mathrm{CH}$-Streckschwingung und dem Oberton der $\mathrm{CH}_{2}$-Knickschwingung sowie der CC-Streckschwingung und dem Oberton der CCO-Knickschwingung, umgekehrt vorausgesagt. Für die MP2-Rechnung ergeben sich außer für die CH-Streckschwingungen und die $\mathrm{CF}_{3}$-Knickschwingungsbande bei $541 \mathrm{~cm}^{-1}$ (exp.) gute Übereinstimmungen. 
Insgesamt konnte für TFE eine vollständige Bandenzuordnung der Fundamentalschwingungen erfolgen, die unter den genannten Näherungen zur Abschätzung der Nullpunktsenergie genutzt wurde. Drei Fermiresonanzen wurden identifiziert und die entstörten Bandenpositionen bestimmt. Die experimentell korrigierten Nullpunktsenergien unterscheiden sich um bis zu $1.2 \mathrm{~kJ} / \mathrm{mol}$ von den berechneten, was für ein relativ starres Molekül bereits eine signifikante Änderung ist.

\subsection{3,3,3-Trifluorpropanol}

Ein interessantes System zur Untersuchung von Isomerisierungen ist 3,3,3-Trifluorpropanol (TFP). In FTIR-Jetexperimenten wurden zwei stabile Monomerkonformere nachgewiesen. ${ }^{60}$ Insgesamt existieren fünf stabile Konformere mit Umwandlungsbarrieren von $400-1200 \mathrm{~cm}^{-1}{ }^{18}$ Somit ist TFP für OPO-Experimente grundsätzlich gut geeignet, sofern der IR-Absorptionsquerschnitt ausreicht.
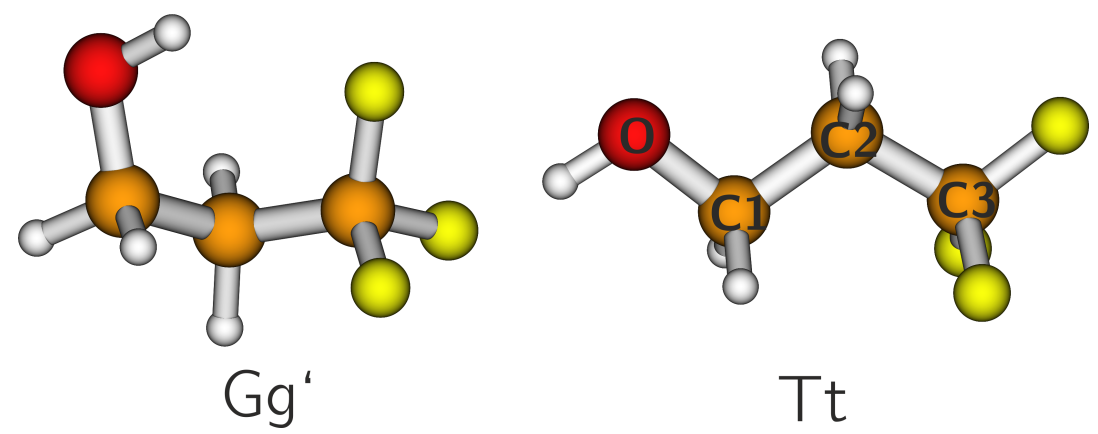

Abbildung 6.4: Die beiden stabilsten Konformere von 3,3,3-Trifluorpropanol. Das Gg'Konformer ist das globale Minimum, beim nächsthöher liegenden Tt-Konformer ist außerdem die für die Nomenklatur der Diederwinkel verwendete Nummerierung der CAtome eingezeichnet. Beide Strukturen wurden auf B3LYP/aVTZ-Niveau berechnet.

Die Konformere von 3,3,3-Trifluorpropanol können durch die zwei Diederwinkel $\tau_{1}$ (C3$\mathrm{C} 2-\mathrm{C} 1-\mathrm{O})$ und $\tau_{2}(\mathrm{C} 2-\mathrm{C} 1-\mathrm{O}-\mathrm{H})$ vollständig charakterisiert werden. Für $\tau_{1}$ werden in der Nomenklatur Großbuchstaben und für $\tau_{2}$ Kleinbuchstaben verwendet. ${ }^{17}$ Bei einem Winkel von $\approx \pm 60^{\circ}$ handelt es sich um eine gauche- ( $\mathrm{G}$ bzw. g) und bei einem Winkel von $\approx 180^{\circ}$ um einen trans-Konformation ( $\mathrm{T}$ bzw. $\mathrm{t}$ ). Das globale Minimum ist das $\mathrm{Gg}^{\prime}$ - Konformer wobei der Strich (," ") hier ausdrückt, dass die beiden Werte der Diederwinkel unterschiedliche Vorzeichen besitzen. Als nächsthöheres folgt das Tt-Konformer. In Abbildung 6.4 sind die beiden Konformere sowie die zur Nomenklatur verwendete Nummerierung der C-Atome gezeigt. 
Der Energieunterschied der beiden stabilsten Konformere wurde bereits experimentell auf 3.5(10) kJ/mol abgeschätzt. ${ }^{142}$ In einer Masterarbeit im Jahr 2012 wurde der Versuch unternommen, diesen Energieunterschied mithilfe von Raman-Jetspektren im OH-Streckschwingungsbereich genauer einzugrenzen. ${ }^{17}$ In der vorliegenden Arbeit wurde der experimentelle Datensatz um zwei weitere spektrale Bereiche erweitert und zwar um den CC-Streckschwingungs- und den CH-Streckschwingungsbereich. Im Folgenden sind die Spektren sowie die Auswertung der experimentellen Spektren, die auch für den schon bekannten $\mathrm{OH}-$ Bereich ergänzt wurde, dargelegt. Die Ergebnisse dieses Kapitels sind bereits in Ref. 18 publiziert.

Die experimentelle Bestimmung des Energieunterschiedes fußt auf der Annahme, dass das Konformerenverhältnis bei der Jetexpansion relativ früh eingefroren wird. ${ }^{143}$ Es kommt also nur in sehr geringem Maße zur Relaxation und das erhaltene Verhältnis entspricht näherungsweise demjenigen, das vor der Expansion eingestellt war. Dieses Modell wurde für Barrieren oberhalb von $400 \mathrm{~cm}^{-1}$ entwickelt, ${ }^{31}$ jedoch wurde kürzlich in Raman-Jetexperimenten ein Relaxationsverhalten bei Alkanen mit Barrierenhöhen von $10 \mathrm{~kJ} / \mathrm{mol}$ gefunden, ${ }^{144}$ so dass ein gewisser Anteil an Relaxation für TFP nicht ausgeschlossen werden kann. Dies gilt insbesondere für die vergleichsweise freie und tunnelunterstützte $\mathrm{OH}$-Torsion, wie das Beispiel Ethanol zeigt. ${ }^{16}$ Durch Nutzung der heizbaren Düse können verschiedene Konformerenverhältnisse vor der Expansion eingestellt werden.

Unter Vernachlässigung jeglicher Strukturrelaxation kann für die Konformerentemperatur $T_{\text {conf }}$ die Düsentemperatur $T_{\mathrm{D}}$ eingesetzt werden. Das Intensitätsverhältnis $F$ einer Tt- zu einer $\mathrm{Gg}^{\prime}$-Bande ist durch das Verhältnis der berechneten Streuquerschnitte $C$ und das Besetzungsverhältnis der Konformere $S$ bestimmt. Vereinfachend wird hier angenommen, dass die Entropiebeiträge der Rotations- und Schwingungsfreiheitsgrade der verschiedenen Konformere zur freien Umwandlungsenthalpie sich aufheben, so dass sich der in Gleichung 6.3 dargestellte Zusammenhang mit der Umwandlungsenthalpie $\Delta H^{\ominus}$ ergibt, wobei der Faktor $\frac{1}{2}$ sich aus der zweifachen Entartung des Gg'-Konformers $(\mathrm{G}(+) \mathrm{g}(-)$ und $\mathrm{G}(-) \mathrm{g}(+))$ ergibt. $^{18}$

$$
\begin{gathered}
F=C \cdot S=\frac{\sigma_{\mathrm{Tt}}^{\prime}}{\sigma_{G g^{\prime}}^{\prime}} \cdot \frac{N(\mathrm{Tt})}{N\left(\mathrm{Gg}^{\prime}\right)}=\frac{\sigma_{\mathrm{Tt}}^{\prime}}{\sigma_{\mathrm{Gg}^{\prime}}^{\prime}} \cdot \frac{1}{2} \exp \left(-\frac{\Delta H^{\ominus}}{R T_{\mathrm{D}}}\right) \\
\ln F=\ln (C / 2)-\frac{\Delta H^{\ominus}}{R T_{\mathrm{D}}}
\end{gathered}
$$

Aus einer Auftragung von $\ln (2 F / C) \times R$ gegen $\frac{1}{T_{\mathrm{D}}}$ kann der Enthalpieunterschied aus der Steigung bestimmt werden.

In Abbildung A.7.1 im Anhang sind Raman-Jetspektren im Bereich von 3050-2850 $\mathrm{cm}^{-1}$ (a) und 1200-600 $\mathrm{cm}^{-1}$ (b) gezeigt. Es wurde jeweils ein gut von anderen Signalen se- 
pariertes $\mathrm{Tt} / \mathrm{Gg}^{\prime}-$ Bandenpaar gefunden: die symmetrische $\mathrm{C} 1-\mathrm{H}-\mathrm{Streckschwingung} \mathrm{bei}$ $2904 \mathrm{~cm}^{-1}\left(\mathrm{Gg}^{\prime}\right)$ und $2919 \mathrm{~cm}^{-1}(\mathrm{Tt})$ und die C2-C3-Streckschwingung bei $797 \mathrm{~cm}^{-1}$ $\left(\mathrm{Gg}^{\prime}\right)$ und $848 \mathrm{~cm}^{-1}(\mathrm{Tt})$. Die Zuordnung der verbleibenden nicht gut separierten Banden sowie Raman-Jetspektren weiterer Spektralbereiche können Ref. 18 entnommen werden. Für die CH-Streckschwingungsbanden kann eine Überlagerung mit Fermiresonanz-verstärkten Obertönen der CH-Knickschwingungen (ähnlich wie bei TFE) nicht ausgeschlossen werden. In Abbildung A.7.2 im Anhang ist daher ein Spektrum aufgeführt, das den Bereich von 1600-1200 $\mathrm{cm}^{-1}$ zeigt. Es wurden zwei Banden bei $1478 \mathrm{~cm}^{-1}$ und $1497 \mathrm{~cm}^{-1}$, deren Obertöne besonders für eine Fermiresonanz mit den CH-Streckschwingungen infrage kämen, identifiziert. Dementsprechend können die Intensitäten der für die Bestimmung des Enthalpieunterschiedes verwendeten CH-Streckschwingungsbanden möglicherweise verfälscht sein.

In Abbildung 6.5, oben sind Raman-Jetspektren der beiden Bandenpaare, die jeweils bei einem Düsenabstand von $1 \mathrm{~mm}$ und bei drei verschiedenen Düsentemperaturen aufgenommen wurden, gegenüber gestellt. Sie wurden auf die $\mathrm{Gg}^{\prime}$-Bandenintensität normiert. Der Effekt der Düsenheizung zeigt sich im Ansteigen der Tt-Bandenintensität relativ zur Gg'-Bande, ist jedoch recht subtil. Bei der CC-Streckschwingung (Abb. 6.5, oben links) zeigt sich blauverschoben zur Gg'-Bande außerdem eine Schulter, die mit steigender Düsentemperatur geringer wird, was auf eine Clusterbande schließen lässt. Zur Bestimmung der Intensitäten wurden vier verschiedene Methoden genutzt: ein Lorentzfits, das Produkt aus Höhe und voller Halbwertsbreite (FWHM) sowie zwei unterschiedliche Integrationsmethoden. Bei einer Integrationsmethode wurde die Gesamtbreite des Integrationsbereichs auf drei volle Halbwertsbreiten der jeweiligen Bande gesetzt, bei der zweiten Methode wurden engere Grenzen gesetzt, so dass ein Sockel von etwa 10\% der gesamten Bandenfläche abgeschnitten wurde. Die erhaltenen Werte sind in Tabelle A.7.2 im Anhang aufgeführt. Die Absolutwerte können erheblich voneinander abweichen, da sich für das jeweilige Bandenpaar jedoch sehr ähnliche Steigungen in den Auftragungen ergaben, wurde mit den Mittelwerten gearbeitet.

Die Auftragung von $\ln (2 F / C) \times R$ gegen $\frac{1}{T_{\mathrm{D}}}$ für drei Bandenpaare, inklusive der Ergebnisse für die OH-Streckschwingung, ist in Abbildung 6.5, unten gezeigt. Die verwendeten Werte für das berechnete Verhältnis der Streuquerschnitte $C^{18}$ sind ebenfalls in Tabelle A.7.2 im Anhang angegeben. Die Fehlergrenzen wurden aus der Standardabweichung der $F$-Werte und den Fehlern der $C$-Werte mittels Fehlerfortpflanzung gemäß dem Größtfehler ermittelt. Für die lineare Regression wurde eine Ursprungsgerade verwendet, d.h. es wird angenommen, dass das berechnete Verhältnis der Streuquerschnitte $C$ korrekt ist.

In Tabelle 6.4 sind die erhaltenen Werte für den Energieunterschied angegeben. Für die drei Bandenpaare ergibt sich eine breite Streuung, was zeigt, dass das verwendete Modell des Einfrierens für TFP nicht unbedingt geeignet ist. Eine besonders große 

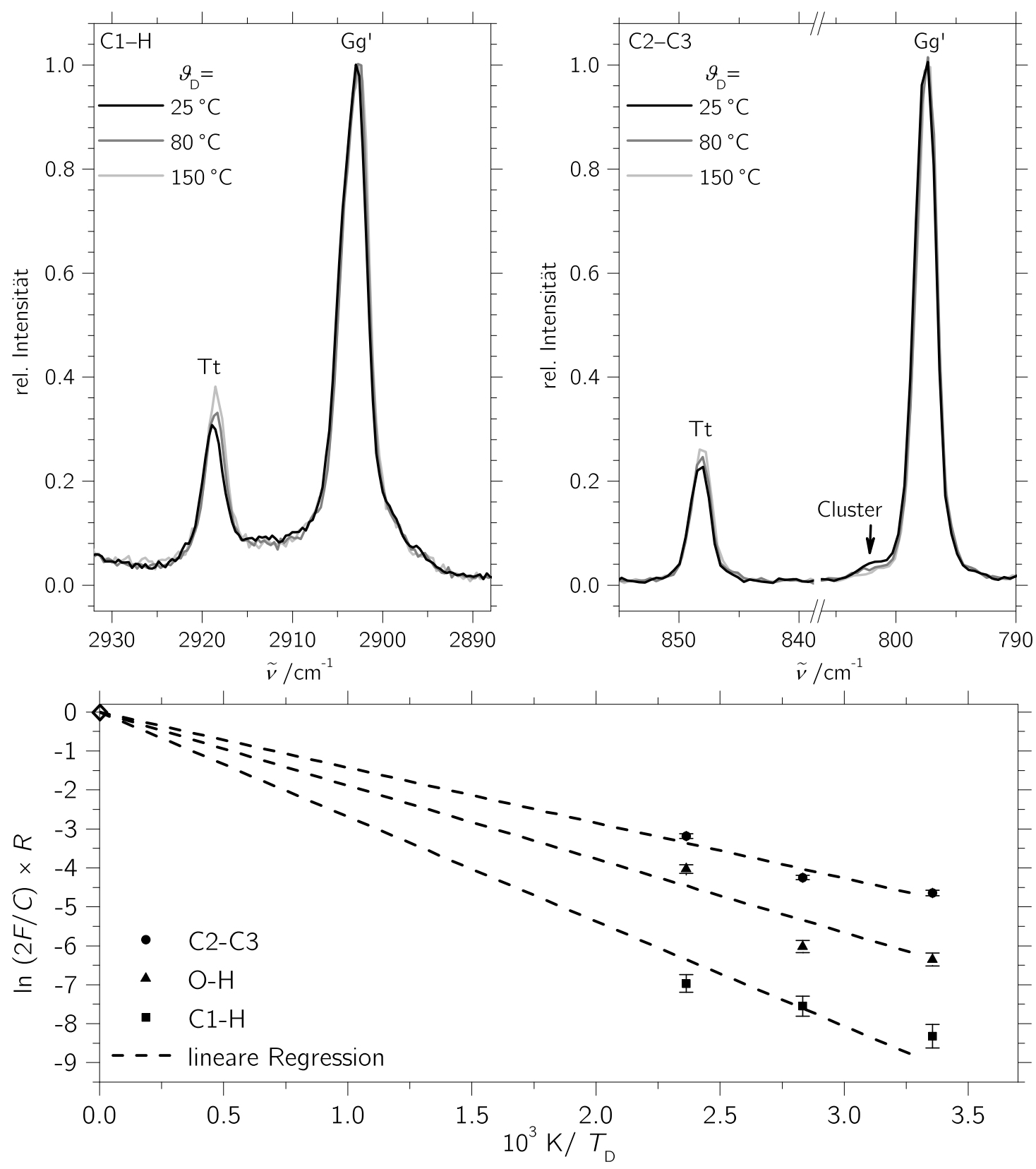

Abbildung 6.5: Oben Links: Symmetrische C1-H-Streckschwingungsbanden, oben rechts: C2-C3-Streckschwingungsbanden des Gg' - und Tt-Konformers von TFP jeweils für drei verschiedene Düsentemperaturen $\left(\vartheta_{\mathrm{D}}\right) .{ }^{18}$ Schwarz: $\vartheta_{\mathrm{D}}=25^{\circ} \mathrm{C}$, dunkelgrau: $\vartheta_{\mathrm{D}}=80^{\circ} \mathrm{C}$, hellgrau: $\vartheta_{\mathrm{D}}=150^{\circ} \mathrm{C}$. Die Spektren wurden auf die $\mathrm{Gg}^{\prime}$-Bande normiert. Der Einfluss der Düsentemperatur auf das Konformerenverhältnis zeigt sich im Anstieg der relativen Intensität der Tt-Bande. Details zu den Messbedingungen finden sich im Anhang A.3. Unten: Auftragung von $\ln (2 F / C) \times R$ gegen $\frac{1}{T_{\mathrm{D}}}$ für die drei Bandenpaare von TFP. Für die lineare Regression wurde angenommen, dass die berechneten Verhältnisse der Streuquerschnitte exakt sind, so dass sich jeweils eine Ursprungsgerade ergibt. ${ }^{18}$ 


\begin{tabular}{cc}
\hline & $\Delta H^{\ominus} / \mathrm{kJ} \mathrm{mol}^{-1}$ \\
\hline $\mathrm{C} 2-\mathrm{C} 3$ & $1.44(5)$ \\
$\mathrm{C} 1-\mathrm{H}$ & $2.7(1)$ \\
$\mathrm{O}-\mathrm{H}$ & $1.9(1)$ \\
\hline
\end{tabular}

Tabelle 6.4: Aus der Auftragung in Abbildung 6.5 bestimmte Enthalpieunterschiede $\Delta H^{\ominus}$ in $\mathrm{kJ} / \mathrm{mol}$ der Konformere $\mathrm{Gg}^{\prime}$ und $\mathrm{Tt}$ von TFP für drei verschiedene Bandenpaare. Die Fehlergrenzen sind in Klammern angegeben

Abweichung von den anderen beiden Werten wird für die CH-Streckschwingung erhalten, was zeigt, dass die oben erwähnte Fermiresonanzüberlagerung sehr wahrscheinlich ist. Daher kann hier das berechnete Verhältnis der Streuquerschnitte nicht als exakt angenommen werden. Eine geringere Abweichung von den anderen zwei Werte wird erhalten wenn der berechnete Streuquerschnitt mit in die lineare Regression einbezogen, also keine Ursprungsgerade erhalten wird (vgl. auch Ref. 18).

In Ref. 18 wurden außerdem weitere Werte unter der Annahme einer partiellen Relaxation erhalten. Des Weiteren wurde die in Kapitel 6.1 vorgestellte Methode zur experimentellen Korrektur der Nullpunktsenergie verwendet um den berechneten Energieunterschied zu korrigieren. Es wurden 8 experimentell bestimmte und für beide Konformere hinsichtlich der Molekülbewegung übereinstimmende Banden einbezogen. Die Korrekturen liegen für beide Konformere mit ca. $0.5 \mathrm{~kJ} / \mathrm{mol}$ in einem ähnlichen Bereich wie für TFE. Bei der Bestimmung des Energieunterschiedes der Konformere hebt sich diese Korrektur jedoch größtenteils auf. ${ }^{18}$ In Kombination mit quantenchemischen Rechnungen auf hohem Niveau konnte somit ein Wert von $1.8(5) \mathrm{kJ} / \mathrm{mol}$ als beste Schätzung erhalten werden. Die Vorgehensweise sowie Details der quantenchemischen Rechnungen können demnächst in Ref. 18 nachgelesen werden.

Für TFP wurden experimentelle Energieunterschiede von $1.4-2.7 \mathrm{~kJ} / \mathrm{mol}$ bestimmt. Eine robuste Abschätzung ist allerdings nur in Kombination mit quantenchemischen Rechnungen auf hohen Niveaus möglich. Das Modell des frühen Einfrierens der Konformerenpopulation ist für TFP aufgrund der recht niedrigen $\mathrm{O}-\mathrm{H}$-Torsionsbarriere nur bedingt geeignet. Ein nächster Schritt könnte der Einsatz von TFP als Testsystem für den OPO-Laser sein. Hierdurch könnten dann möglicherweise auch die drei anderen TFP-Konformere im Jet präpariert werden. Hinweise auf das energetisch nächsthöhere Tg-Konformer wurden im OH-Streckschwingungsbereich bereits in Gasphasenspektren sowie in warmen Jetexpansionen gefunden. ${ }^{18}$ 


\subsection{N-Methylformamid}<smiles>CNC=O</smiles>

trans-NMF<smiles>CNC=O</smiles>

cis-NMF

Abbildung 6.6: Die beiden stabilsten Monomerkonformere von $N$-Methylformamid.

Zur Evaluierung der experimentellen Bestimmung des Energieunterschiedes wurde als weiteres System $N$-Methylformamid (NMF) gewählt. Dieses liegt in zwei spektrokopisch gut unterscheidbaren Monomerkonformeren ${ }^{145,146}$ vor, der trans-Form, bei der die $\mathrm{NH}$ und $\mathrm{C}=\mathrm{O}$ Gruppen entgegengesetzt ausgerichtet sind und der etwas instabileren cis-Form. Diese sind in Abbildung 6.6 gezeigt. Die verwendete Nomenklatur ist in Analogie zu vorherigen Jet-FTIR-Arbeiten gewählt, ${ }^{146,147}$ in weiteren Literaturquellen werden jedoch auch andere Konventionen verwendet. ${ }^{148}$

Eine Enthalpiedifferenz der Konformere von $5.4^{149}$ bzw. $7.5(7) \mathrm{kJ} / \mathrm{mol}^{150}$ wurde bereits aus IR-Matrixisolationsspektren bestimmt. Die Differenz der freien Enthalpie wurde in Gasphase $^{151}$ und Lösung in Wasser ${ }^{152}$ auf $5.4 \mathrm{~kJ} / \mathrm{mol}$ und außerdem aus quantenchemischen Rechnungen auf B3LYP/aVTZ-Niveau auf $6.6 \mathrm{~kJ} / \mathrm{mol}$ abgeschätzt. ${ }^{153}$

Die Bestimmung der Energiedifferenz in Jetspektren, in denen keine Einflüsse von Lösungsmitteln sowie keine thermische Verbreiterung der Banden auftreten, blieb jedoch aufgrund der geringen Signalstärke des cis-Konformers im IR-Spektrum ${ }^{147}$ aus. Mittels Raman-Jetspektren bei unterschiedlichen Düsentemperaturen und unterschiedlichen Konzentrationen kann diese Lücke im folgenden Kapitel geschlossen werden.

Im Gegensatz zu TFP wird hier jedoch die freie Enthalpiedifferenz bestimmt. Ein Vergleich mit quantenchemischen Rechnungen auf MP2/aVTZ-Niveau liefert bereits für eine Temperatur von $298 \mathrm{~K}$ eine Differenz zwischen freier Enthalpie $\left(\Delta G^{\ominus}\right)$ und Enthalpie $\left(\Delta H^{\ominus}\right)$ von $2.35 \mathrm{~kJ} / \mathrm{mol}$, so dass die Entropie für dieses System nicht vernachlässigbar ist. Es ergibt sich der folgende Zusammenhang:

$$
\begin{gathered}
F=C \cdot S=\frac{\sigma_{\text {cis }}^{\prime}}{\sigma_{\text {trans }}^{\prime}} \cdot \frac{N(\text { cis })}{N(\text { trans })}=\frac{\sigma_{\text {cis }}^{\prime}}{\sigma_{\text {trans }}^{\prime}} \cdot \exp \left(-\frac{\Delta G^{\ominus}}{R T_{\mathrm{D}}}\right) \\
\Delta G^{\ominus}=-R T_{\mathrm{D}} \cdot \ln \left(\frac{F}{C}\right)
\end{gathered}
$$


Analog zu TFP beschreibt $F$ das Intensitätsverhältnis der Banden, $C$ das Verhältnis der Streuquerschnitte und $S$ das Besetzungsverhältnis.

Aufgrund des Doppelbindungscharakters der Amidbindung ist die Barriere zwischen den beiden Monomerkonformeren von NMF sehr hoch. Sie wird zu $89 \mathrm{~kJ} / \mathrm{mol}$ auf B3LYP/6-311+G*-Niveau vorhergesagt. ${ }^{\text {145,146 }}$ Dementsprechend kann eine Relaxation im Jet ausgeschlossen werden und das Modell des Einfrierens der Konformerenpopulation kann als gültig angesehen werden, so dass als Temperatur die Düsentemperatur $\left(T_{\mathrm{D}}\right)$ in Gleichung 6.5 eingesetzt werden kann. Hierbei wird angenommen, dass eine vollständige Gleichgewichtseinstellung bis zur Düsenspitze erfolgt.

Zur Berechnung der Streuquerschnitte wurden quantenchemische Rechnungen auf B3LYP/6-311++G(d,p)-, B3LYP/aVTZ- und MP2/aVTZ-Niveau durchgeführt. Bei den Minimumsstrukturen ist die Konformation der $\mathrm{CH}_{3}$-Gruppe aufgrund ihrer geringen Rotationsbarriere von ca. $0.7 \mathrm{~kJ} / \mathrm{mol}^{148,154}$ nicht geklärt. ${ }^{146,155}$ Auf den verwendeten Niveaus ergaben sich jedoch nur für das gestaffelte trans- und das ekliptische cis-Konformer stabile Strukturen. Die subtile Konformationsisomerie der Methylgruppe ist auf den genannten Niveaus aufgrund der geringen Barriere nur schwer zu erfassen. Bei MP2-Rechnungen mit kleinen Basissätzen kann durch den intramolekularen Basissatzüberlagerungsfehler (BSSE) auch eine Umkehr der vorhergesagten Energieabfolge in Bezug auf die Konformation der Methylgruppe erfolgen, was für das strukturell dem NMF sehr ähnliche $N$-Methylacetamid gezeigt werden konnte. ${ }^{156}$ Der Einfluss der Konformation der Methylgruppe auf die verwendeten Streuquerschnitte der NHStreckschwingung ist jedoch vernachlässigbar, was die alleinige Verwendung der zuvor genannten Minimumsstrukturen rechtfertigt. Im Experiment könnten entweder jeweils beide cis- und trans-Formen als Gemisch vorliegen oder aber, für den Fall, dass jeweils nur eine Form ein Minimum ist, nur diese. Generell ist die jeweilige ekliptische von der gestaffelten Form aber bei der gegebenen Auflösung spektroskopisch nicht unterscheidbar. Die erhaltenen Werte für die Streuquerschnitte sowie der für die weiteren Berechnungen verwendete Mittelwert sind in Tabelle A.7.3 angegeben. Als Fehler wurde eine Standardabweichung genutzt.

Es wurden Raman-Jetspektren unter Nutzung der heizbaren Düse in verschiedenen Konzentrationen aufgenommen, und zwar bei einem Abstand von $1 \mathrm{~mm}$ einmal bei sehr niedriger Konzentration $\left(\vartheta_{\mathrm{S}}=5^{\circ} \mathrm{C}\right)$ und bei höherer Konzentration $\left(\vartheta_{\mathrm{S}}=18^{\circ} \mathrm{C}\right)$ sowie bei einem Abstand von $2 \mathrm{~mm}$, bei dem die Dimerkonzentration $\left(\vartheta_{\mathrm{S}}=12^{\circ} \mathrm{C}\right)$ ähnlich derjenigen bei $1 \mathrm{~mm}$ Abstand und $18^{\circ} \mathrm{C}$ Sättigertemperatur war.

Beispielhaft sind in Abbildung 6.7 drei Spektren gezeigt, die jeweils bei einer Sättigertemperatur von $12^{\circ} \mathrm{C}$ und einem Abstand zur Düse von $2 \mathrm{~mm}$ aufgenommen wurden. Es wurden Düsentemperaturen von $25^{\circ} \mathrm{C}$ (schwarz), $80^{\circ} \mathrm{C}$ (grau) und $150{ }^{\circ} \mathrm{C}$ (hellgrau) eingesetzt. Die Spektren sind normiert auf die dominante Bande bei $3501 \mathrm{~cm}^{-1}$, die in Übereinstimmung mit IR-Jetspektren ${ }^{145-147}$ der NH-Streckschwingung des transMonomers zugeordnet wurde. Leicht rotverschoben zum trans-Monomer befindet sich 


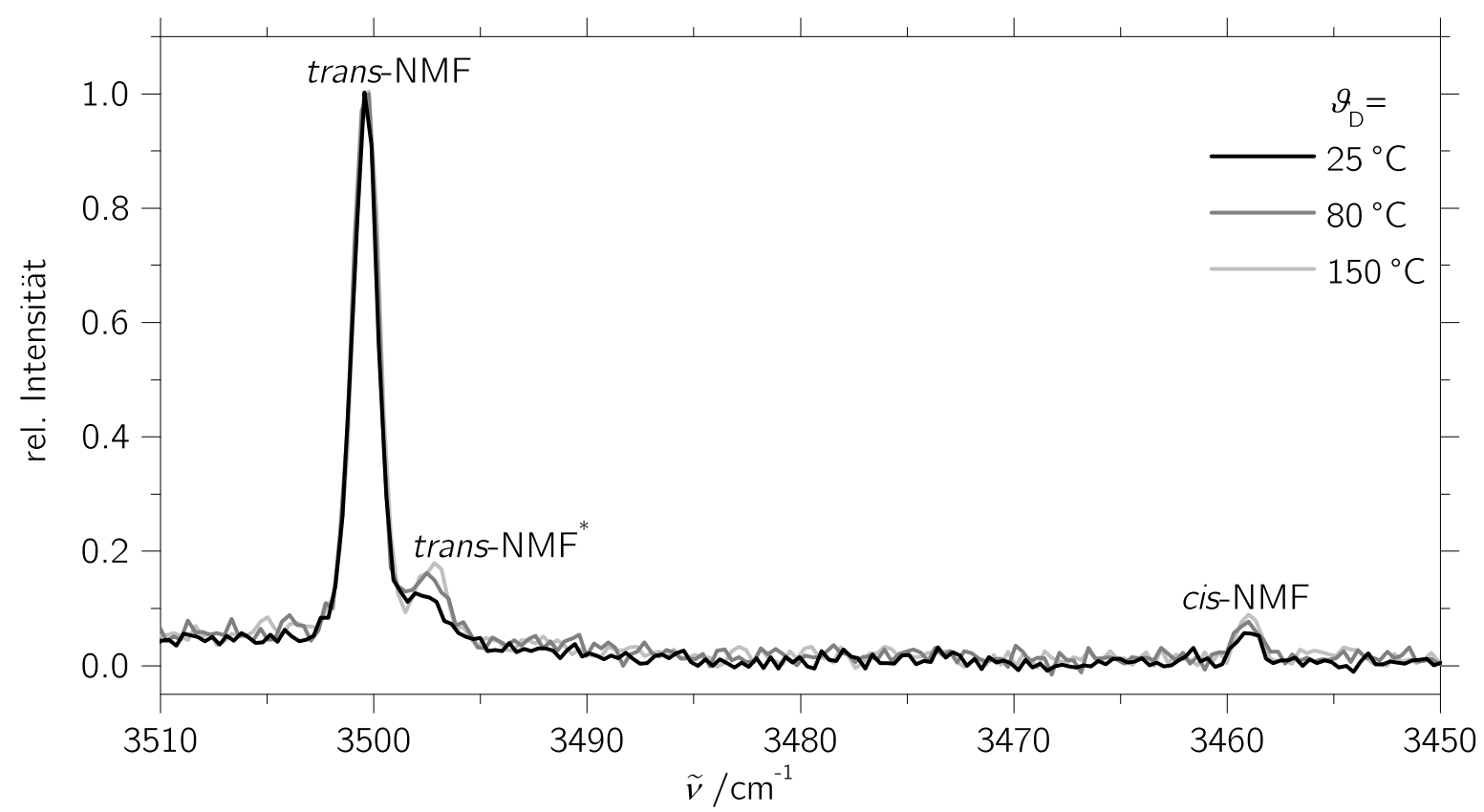

Abbildung 6.7: Bei Düsentemperaturen von $25^{\circ} \mathrm{C}$ (schwarz), $80^{\circ} \mathrm{C}$ (grau) und $150{ }^{\circ} \mathrm{C}$ (hellgrau) aufgenommene Raman-Jetspektren des NH-Streckschwingungsbereichs von NMF bei einem Düesnabstand von $2 \mathrm{~mm}$ und einer Sättigertemperatur von $12{ }^{\circ} \mathrm{C}$. Die Spektren sind auf die dominante Bande des trans-Monomers normiert. Es zeigt sich eine Nebenbande (trans-NMF*), die auf eine heiße Bande von trans-NMF zurückgeführt werden kann. Außerdem ist die Bande des cis-Monomers zu erkennen. Weitere Angaben zu den Messbedingungen sind im Anhang A.3 aufgeführt.

bei $3497 \mathrm{~cm}^{-1}$ eine weitere Bande, die mit steigender Temperatur anwächst. Sie wurde auf eine heiße Bande des trans-Monomers zurückgeführt. Höchstwahrscheinlich handelt es sich um Monomere, bei denen die $\mathrm{CH}_{3}$-Torsion angeregt ist. In Abbildung A.7.3 im Anhang ist gezeigt, wie stark die Bande relativ zur trans-Monomerfundamentalen mit steigender Temperatur anwächst. Es ist jeweils ein Spektrum bei niedriger (grau) und bei hoher (schwarz) Konzentration gezeigt. Da sich bei hoher Konzentration vermehrt Cluster ausbilden, deren Kondensationswärme von Trägergas und Monomeren abgeführt wird, ist die Monomerschwingungstemperatur hier höher. Die relative Intensität der Bande bei $3497 \mathrm{~cm}^{-1}$ verdreifacht sich vom niedrig- zum hochkonzentrierten Spektrum. Sie ist somit eine nützliche Sonde für die trans-Monomerschwingungstemperatur.

Bei $3458 \mathrm{~cm}^{-1}$ befindet sich eine weitere Bande, die ebenfalls mit steigender Temperatur anwächst. Diese wurde auf das cis-Monomer zurückgeführt. Hier ergibt sich jedoch eine Diskrepanz zu IR-Ne-Matrixspektren und FTIR-Jetspektren, bei denen die cis-Monomerbande bei $3452 \mathrm{~cm}^{-1}$ gefunden wurde. ${ }^{147,157}$ Da sowohl die transMonomer- als auch die trans-trans-Dimerbande mit den FTIR-Werten übereinstimmen, kann ein Kalibrierfehler aber ausgeschlossen werden. Im FTIR-Jetspektrum ist 


\begin{tabular}{ccccccc}
\hline \multicolumn{7}{c}{$\Delta G^{\ominus} / \mathrm{kJ} \mathrm{mol}^{-1}$} \\
\hline$T / \mathrm{K}$ & $\begin{array}{c}\vartheta_{\mathrm{S}}=5^{\circ} \mathrm{C}, \\
d=1 \mathrm{~mm}\end{array}$ & $\begin{array}{c}\vartheta_{\mathrm{S}}=12^{\circ} \mathrm{C}, \\
d=2 \mathrm{~mm}\end{array}$ & $\begin{array}{c}\vartheta_{\mathrm{S}}=18^{\circ} \mathrm{C}, \\
d=1 \mathrm{~mm}\end{array}$ & $\begin{array}{c}\text { B3LYP } / \\
6-311++\mathrm{G}(\mathrm{d}, \mathrm{p})\end{array}$ & $\begin{array}{c}\text { B3LYP } / \\
\mathrm{aVTZ}\end{array}$ & $\begin{array}{c}\text { MP2/ } \\
\mathrm{aVTZ}\end{array}$ \\
\hline 298 & $6.9(4)$ & $7.1(5)$ & $7.6(5)$ & 6.7 & 6.2 & 8.7 \\
323 & & & $8.3(6)$ & 6.9 & 6.4 & 8.9 \\
353 & & $8.0(6)$ & & 7.2 & 6.6 & 9.2 \\
373 & & & \multirow{2}{*}{$8.9(6)$} & 7.3 & 6.8 & 9.3 \\
423 & & $8.9(6)$ & & 7.7 & 7.1 & 9.7 \\
433 & $9.7(6)$ & & & 7.8 & 7.2 & 9.8 \\
\hline
\end{tabular}

Tabelle 6.5: Experimentell ermittelte freie Standardenthalpien $\Delta G^{\ominus}$ in kJ/mol für die Umwandlung von trans- in cis-NMF im Vergleich zu theoretisch ermittelten Werten für verschiedene Düsentemperaturen $T_{\mathrm{D}}$. Mit steigender Temperatur erhöht sich auch die freie Enthalpie, was die theoretischen Vorhersagen bestätigen. Relative Intensitäten finden sich in Tabelle A.7.4 im Anhang.

die cis-Monomerbande außerdem extrem schwach, daher ist dort die Zuordnung eher unzuverlässig.

Zur Bestimmung der freien Enthalpie wurden die cis- sowie beide trans-Banden in den jeweiligen Spektren integriert. Die Werte sind in Tabelle A.7.4 im Anhang aufgeführt. Die bestimmten freien Enthalpien sind in Tabelle 6.5 angegeben, wobei für die Messungen bei $5{ }^{\circ} \mathrm{C}$ Sättigertemperatur, für die es drei oder mehr Resultate für die einzelnen Düsentemperaturen gibt, die Mittelwerte angegeben wurden. Zur Fehlerabschätzung wurde eine Fehlerfortpflanzung nach dem Größtfehler durchgeführt, wobei für das Intensitätsverhältnis $F$ ein Fehler von $10 \%$, für das Verhältnis der Streuquerschnitte die Standardabweichung vom Mittelwert (s. auch Tabelle A.7.4 im Anhang) und für die Düsentemperatur ein Fehler von $10 \mathrm{~K}$ angenommen wurden. Für Messungen mit drei oder mehr Messwerten wurde der Mittelwert der Größtfehler angegeben. Zum Vergleich sind in Tabelle 6.5 außerdem berechnete Werte aufgeführt. Innerhalb der jeweiligen Messreihe zeigt sich, dass die freie Enthalpie mit zunehmender Temperatur ansteigt. Dieser Trend wird von den quantenchemischen Rechnungen bestätigt, jedoch sind hier die absoluten Differenzen geringer. Es fällt aber eine Differenz von $0.4 \mathrm{~kJ} / \mathrm{mol}$ zwischen dem B3LYP/aVTZ-Wert (bei $298 \mathrm{~K}$ ) aus Ref. 153 und dem in Tabelle A.7.4 angegebenen auf, die wahrscheinlich darauf zurückzuführen ist, dass dort das gestaffelte cis-Isomer verwendet wurde.

In Abbildung 6.8 sind die berechneten und experimentell bestimmten freien Standardenthalpien gegen die absolute Temperatur aufgetragen. Es wurden die Messwerte der drei verschiedenen Messbedingungen gemeinsam aufgetragen. Für die Temperatur von $298 \mathrm{~K}$ wurde der Mittelwert (7.1(5) kJ/mol) gebildet und als Fehler wurde jeweils der Größtfehler verwendet. Im Rahmen der Fehlergrenzen ergibt sich aus dem Experiment 


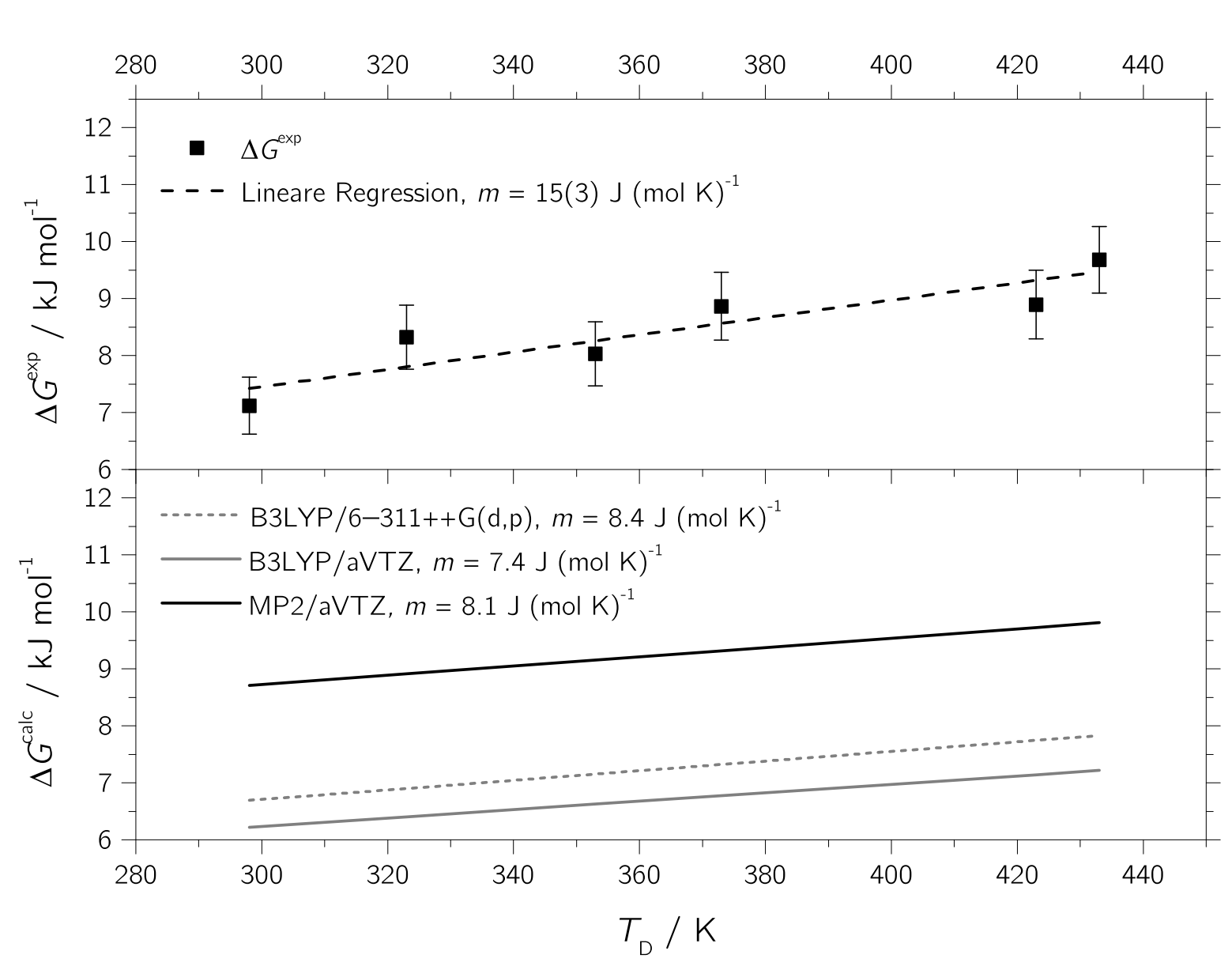

Abbildung 6.8: Auftragung der experimentell und aus quantenchemischen Rechnungen bestimmte freie Standardenthalpien gegen die Temperatur. Aus einer linearen Regression ergibt sich aus der negativen Steigung $m$ die Standardentropie $\Delta S^{\ominus}$. Es zeigt sich, dass der Betrag der Entropie von den harmonischen Rechnungen deutlich unterschätzt wird. Berechnete Enthalpie- und Entropiewerte finden sich in Tabelle A.7.5 im Anhang.

ein lineares Verhalten der freien Enthalpie mit der Temperatur. Aus einer linearen Regression kann somit aus der negativen Steigung die Entropie bestimmt werden. Es zeigt sich, dass die Steigung $m$ für die experimentellen Werte mit $15(3) \mathrm{J} \mathrm{mol}^{-1} \mathrm{~K}^{-1}$ ungefähr doppelt so groß ist wie die für die berechneten Werte. Der Betrag der Entropie wird also von den harmonischen Rechnungen deutlich unterschätzt. Dies setzt voraus, dass eine vollständige Gleichgewichtseinstellung bis zur Düsenspitze erfolgt ist. Die zugehörigen berechneten Enthalpie- und Entropiewerte finden sich in Tabelle A.7.5 im Anhang. Hier zeigt sich zudem, dass die Änderung von Enthalpie und Entropie mit der Temperatur im betrachteten Temperaturbereich vernachlässigbar klein ist. Außerdem ergeben sich besonders für die Enthalpiewerte Abweichungen zwischen den verschiedenen Rechenmethoden während die Entropiewerte gut übereinstimmen. 
Beim Vergleich zwischen den Messreihen besonders bei Düsentemperaturen oberhalb der Raumtemperatur zeigen sich Diskrepanzen, die einer näheren Analyse bedürfen. Beispielsweise wurde für die Düsentemperaturen von $373 \mathrm{~K}\left(\vartheta_{\mathrm{S}}=18^{\circ} \mathrm{C}, 1 \mathrm{~mm}\right)$ und $423 \mathrm{~K}\left(\vartheta_{\mathrm{S}}=12^{\circ} \mathrm{C}, 2 \mathrm{~mm}\right)$ jeweils ein Wert von $8.9(6) \mathrm{KJ} / \mathrm{mol}$ ermittelt. Der Wert für eine Temperatur von $353 \mathrm{~K}$ ist sogar geringer als der für $323 \mathrm{~K}$, wobei die Werte unter Berücksichtigung der Fehlergrenzen auch invertiert sein können. Da dieser Befund möglicherweise auf den Einfluss der Dimerbildung zurückgeführt werden kann, wurde zunächst der Dimeranteil des trans-trans-Dimers aus dem Intensitätsverhältnis von Monomer- und Dimerbande abgeschätzt. Es ergibt sich ein maximaler Anteil von 3\%, der dementsprechend vernachlässigbar ist.

Ein weiterer Grund für die Diskrepanzen könnte jedoch eine verstärkte Ausbildung der sehr viel stabileren, cyclischen cis-cis-Dimere $^{146}$ sein, wodurch ein systematischer Fehler entstehen würde. Erste Anzeichen für die Bildung eines cis-cis-Dimers wurden bereits in FTIR-Jetspektren gefunden. ${ }^{147}$ Es wurde eine sehr schwache Bande bei $3177 \mathrm{~cm}^{-1}$ identifiziert.

Um einen möglichst hohen Anteil an cis-Monomeren vor der Expansion zu generieren wurde der beheizbare Sättiger bei einer Temperatur von $60^{\circ} \mathrm{C}$ genutzt. In Abbildung 6.9 , oben sind drei Spektren bei Düsentemperaturen von $80^{\circ} \mathrm{C}, 120^{\circ} \mathrm{C}$ und $150^{\circ} \mathrm{C}$ gezeigt. Hier wurden die Spektren ebenfalls auf die trans-Monomerbande normiert. Neben der cis-Monomerbande (c) und der trans-trans-Dimerbande (tt) zeigt sich noch eine breite Signatur, die ähnlich bereits in FTIR-Jetspektren gefunden und auf große Cluster zurückgeführt wurde. ${ }^{146}$ Außerdem ist ein scharfer Peak bei $3113 \mathrm{~cm}^{-1}$, der aufgrund seiner Rotverschiebung als cis-cis-Dimerbande infrage käme, zu erkennen. Im unteren Teil der Abbildung ist das Spektrum, das bei $60{ }^{\circ} \mathrm{C}$ Sättiger- und $80^{\circ} \mathrm{C}$ Düsentemperatur aufgenommen wurde, im Vergleich mit einem sehr viel schwächer konzentrierten Spektrum, das bei einer Sättigertemperatur von $15^{\circ} \mathrm{C}$ und ungeheizter Düse aufgenommen wurde, gezeigt. Es zeigt sich, dass sich die Position der trans-trans-Dimerbande bei hoher Konzentration blauverschiebt, was auf Überlagerungen mit größeren Clustern oder eine thermische Schwächung der isolierten Wasserstoffbrückenbindung zurückzuführen ist.

Auch bei extrem geringer Konzentration bleibt die Bande bei $3113 \mathrm{~cm}^{-1}$ erhalten. Das könnte auf ein extrem stabiles, kleines Aggregat hinweisen. Gegen diese Vermutung spricht jedoch, dass die Bande nicht mit der cis-Monomerkonzentration wächst, sondern in allen Spektren etwa gleich stark ist. Dieses Verhalten schließt auch ein cistrans-Dimer aus.

Eine Abschätzung der zu erwartenden Intensität des cis-cis-Dimers (cc) relativ zum trans-trans-Dimer (tt) kann gemäß Gleichung 6.7 aus dem Besetzungsverhältnis der Monomerbanden und den Streuquerschnitten der Dimerbanden erhalten werden. 


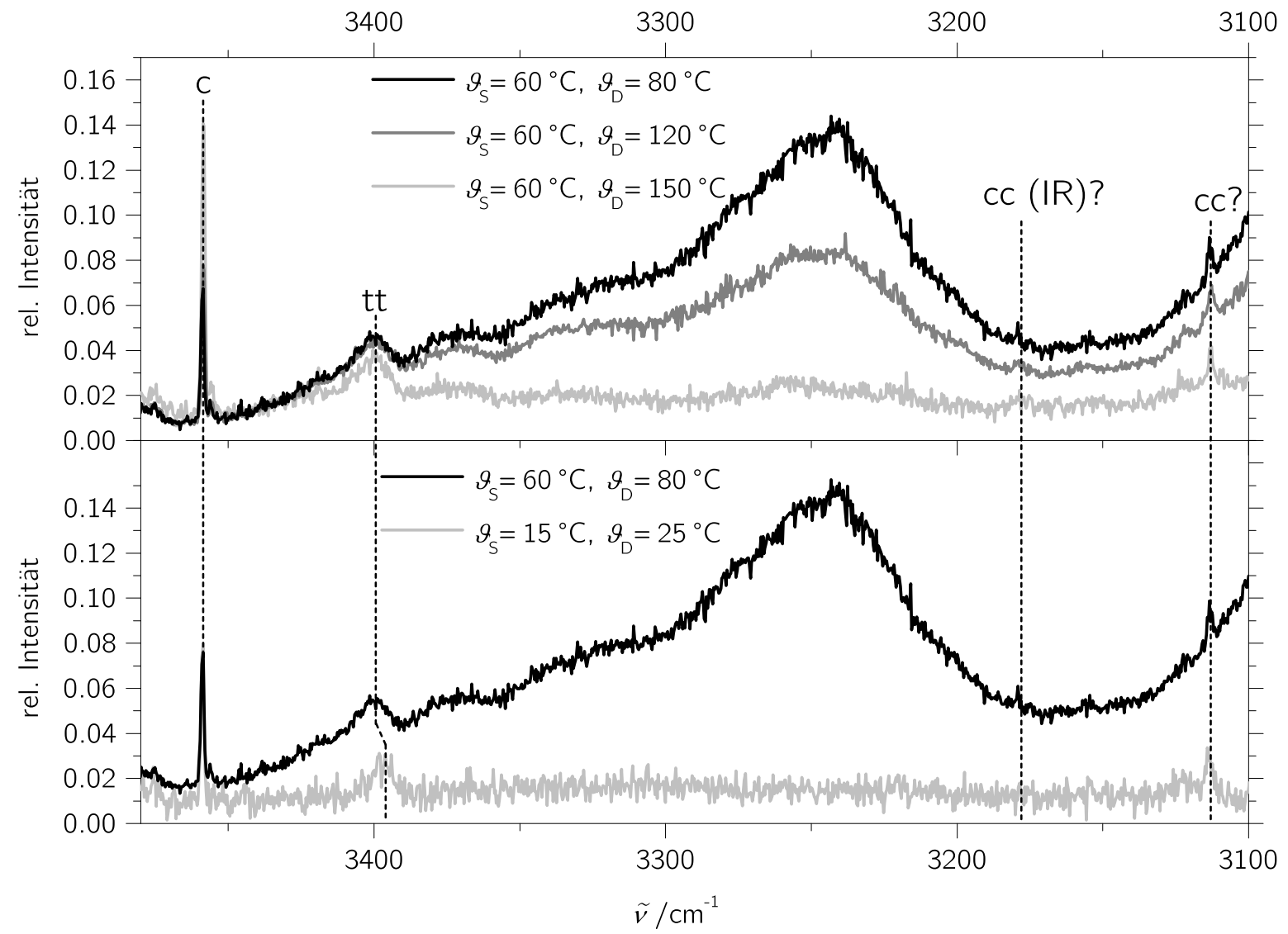

Abbildung 6.9: Oben: Unter Nutzung des heizbaren Sättigers aufgenommene Spektren von NMF bei Düsentemperaturen von $80^{\circ} \mathrm{C}$ (schwarz), $120^{\circ} \mathrm{C}$ (grau) und $150^{\circ} \mathrm{C}$ (hellgrau). Die Spektren sind auf die trans-Monomerbande normiert. Neben dem cis-Monomer (c) und dem trans-trans-Dimer (tt) wurde außerdem eine Bande bei $3113 \mathrm{~cm}^{-1}$ gefunden, die möglicherweise als cis-cis-Dimerbande infrage kommt (cc?). Des Weiteren wurde eine Bande bei $3177 \mathrm{~cm}^{-1}$ (cc(IR)?) gefunden, die bereits im FTIR-Jetspektrum vorsichtig dem cis-cis-Dimer zugeordnet wurde. ${ }^{147}$ Unten: Vergleich des Spektrums bei $80^{\circ} \mathrm{C}$ Düsentemperatur (schwarz) mit einem wesentlich geringer konzentrierten bei ungeheizter Düse (hellgrau). Neben den mit c und tt bezeichneten Banden bleibt die Bande bei $3113 \mathrm{~cm}^{-1}$ erhalten. Details zu den Messbedingungen s. Tabelle A.3 im Anhang. 


$$
\frac{I(c c)}{I(t t)}=\frac{\sigma_{c c}^{\prime}}{\sigma_{t t}^{\prime}} \cdot\left(\frac{N(c)}{N(t)}\right)^{2}=C_{\mathrm{cc} / \mathrm{tt}} \cdot\left(\frac{N(c)}{N(t)}\right)^{2}
$$

Für die Streuquerschnitte wurden hier quantenchemische Rechnungen auf B3LYP/6-311++G(d,p)- und B3LYP/aVTZ-Niveau durchgeführt und der Mittelwert des Streuquerschnittsverhältnis $\left(C_{\mathrm{cc} / \mathrm{tt}}\right)$ gebildet. Die Ergebnisse sind in Tabelle A.7.3 im Anhang aufgeführt. In Tabelle 6.6 sind die relativen Intensitäten der Monomere sowie die zu erwartende relative Intensität der Dimere für die drei Spektren aus Abbildung 6.9, oben angegeben. Es zeigt sich, dass die cis-cis-Dimerbande um 2-3 Größenordnungen schwächer sein müsste als die trans-trans-Dimerbande, so dass sie in den vorliegenden Spektren nicht vom Rauschen zu unterscheiden wäre.

\begin{tabular}{ccccccccc}
\hline$T_{\mathrm{D}} / \mathrm{K}$ & $I(t)$ & $I(c)$ & $I(t t)$ & $I(c) / I(t)=F$ & $N(c) / N(t)$ & $I(t t) / I(t)$ & $C_{\mathrm{cc} / \mathrm{tt}}$ & $I(c c) / I(t t)$ \\
\hline 353 & 2.59 & 0.08 & 0.14 & $3.3 \cdot 10^{-2}$ & $3.8 \cdot 10^{-2}$ & $5.6 \cdot 10^{-2}$ & 1.51 & $2.2 \cdot 10^{-3}$ \\
393 & 2.60 & 0.11 & 0.14 & $4.2 \cdot 10^{-2}$ & $4.9 \cdot 10^{-2}$ & $5.5 \cdot 10^{-2}$ & & $3.7 \cdot 10^{-3}$ \\
423 & 2.57 & 0.21 & 0.19 & $8.3 \cdot 10^{-2}$ & $9.6 \cdot 10^{-2}$ & $7.4 \cdot 10^{-2}$ & & $1.4 \cdot 10^{-2}$ \\
\hline
\end{tabular}

Tabelle 6.6: Abschätzung der zu erwartenden relativen Intensität des cis-cis- zum transtrans-Dimer $I(c c) / I(t t)$ aus dem experimentellen Besetzungsverhältnis der Monomere $(N(c) / N(t))$ und dem Verhältnis der Streuquerschnitte $C(c c / t t)$ sowie die relativen Intensitäten der Monomere $(F=I(c) / I(t))$ inklusive der ermittelten Integrale $(I(c), I(t))$ für die drei Spektren in Abbildung 6.9, oben. Zur Veranschaulichung ist außerdem das Intensitätsverhältnis der im Spektrum gut erkennbaren trans-trans-Dimerbande zur transMonomerbande $(I(t t) / I(t))$ angegeben. Die cis-cis-Dimerbande müsste um 2-3 Größenordnungen schwächer als die trans-trans-Dimerbande sein und wäre somit vom Rauschen nicht zu unterscheiden.

Außerdem ist die betrachtete Bande relativ schmal, was ebenfalls gegen ein Aggregat spricht. Es handelt sich daher wahrscheinlich um eine trans-Monomerbande. Der erste Oberton der Carbonylstreckschwingung kann ausgeschlossen werden, da die Fundamentale mit $1732 \mathrm{~cm}^{-1}$ (FTIR-Jet) ${ }^{145,146} \mathrm{zu}$ hoch liegt. (Es würde sich eine Anharmonizitätskonstante von $-176 \mathrm{~cm}^{-1}$ ergeben.)

Es könnte sich allerdings um eine Kombinationsbande handeln. In Abbildung A.7.4 im Anhang ist das Raman-Spektrum im Bereich von $1800-1380 \mathrm{~cm}^{-1}$ gezeigt. Neben den schon in Ref. 145 aufgeführten Carbonylstreckschwingungsbanden von Monomeren, Dimeren und Clustern findet sich eine dominante Bande bei $1386 \mathrm{~cm}^{-1}$, die der $\mathrm{CH}-$ Knickschwingung des Aldehyd-H-Atoms zugeordnet werden kann. Die Kombination dieser Bande mit der Carbonylstreckschwingungsbande führt zu einer Wellenzahl von $3118 \mathrm{~cm}^{-1}$ und ist somit die bislang plausibelste Interpretation. Allerdings kommen möglicherweise auch weitere Kombinationsbanden bspw. eine Kombination einer 
CH-Streckschwingungsbande mit der Methyltorsion infrage. Ohne eine Kenntnis der Bandenpositionen im Jet ist aber hier keine definitive Aussage möglich.

In Abbildung 6.9, oben ist besonders im grauen Spektrum außerdem die bereits in FTIR-Spektren gefundene Bande bei $3177 \mathrm{~cm}^{-1}$ schwach zu erkennen. Aufgrund des Alternativverbots, das für das $C_{2 h}$-symmetrische cis-cis-Dimer gilt, kann die IR-aktive NH-Streckschwingungsbande des Dimers nicht im Raman-Spektrum auftreten. Dementsprechend ist die gefundene Bande nicht auf ein cis-cis-Dimer zurückzuführen.

Das Vorliegen eines signifikanten Anteils an cis-cis-Dimer kann somit ausgeschlossen werden. Die Diskrepanzen der freien Enthalpien sind also nicht auf Dimerbildung zurückzuführen, sondern spiegeln im Wesentlichen die Genauigkeit der Methode wider.

Für NMF konnten experimentell freie Enthalpien für die Umwandlung von trans- in cis-NMF für verschiedene Temperaturen ermittelt werden. Diese steigen mit steigender Temperatur; ein Trend der auch theoretisch vorhergesagt wird und auf die niedrigere Entropie des cis-Monomers zurückzuführen ist. Außerdem konnte die Standardentropie ermittelt werden, deren Betrag von den harmonischen Rechnungen deutlich kleiner vorhergesagt wird. Hinweise auf das extrem stabile cis-cis-Dimer konnten jedoch nicht gefunden werden.

\subsection{Zusammenfassung und Ausblick}

Es wurden drei verschiedene Systeme untersucht. Für TFE wurde durch vollständige Zuordnung des Raman-Schwingungsspektrums eine experimentell korrigierte Nullpunktsenergie ermittelt. Für TFP und NMF wurde eine experimentelle Methode zur Ermittlung der Energieunterschiede der verschiedenen Konformere erprobt. Für TFP ist jedoch eine realistische Abschätzung nur unter Zuhilfenahme eines Relaxationsmodells sowie quantenchemischer Rechnungen auf hohem Niveau möglich. ${ }^{18}$

Für Systeme, mit hohen Konversionsbarrieren wie NMF ist die Methode besser geeignet, sollte jedoch noch an weiteren Schwingungsbandenpaaren erprobt werden. Außerdem wäre eine Untersuchung niederfrequenter Banden sinnvoll, um die bei $3113 \mathrm{~cm}^{-1}$ gefundene Bande schlüssiger interpretieren zu können. Die Entwicklung des cis/transVerhältnisses von NMF im thermischen Gleichgewicht soll zudem in einem heizbaren Strömungsrohr untersucht werden. Möglicherweise kann hierdurch der cis-Anteil signifikant erhöht und das cis-cis-Dimer eindeutig identifiziert werden. Um die Konformationsisomerie der Methylgruppe genauer zu beschreiben wäre außerdem der Einsatz lokaler MP2-Methoden sinnvoll.

Die Konformerenvielfalt von TFP soll außerdem mittels OPO-Laseranregung weiter untersucht werden, sofern die IR-Strahlungsdichte dafür ausreicht. 


\section{IR-Laseranregung am Beispiel des Methanol-Dimers}

Methanol ist als einfachster Alkohol gut geeignet als Ausgangssystem für eine IRLaseranregung. Das Monomer besitzt allerdings aufgrund der starken Kopplung der $\mathrm{OH}-$ Streckschwingung zur OH- bzw. Methyltorsion sowie zur Gesamtrotation des Moleküls eine komplexe und sehr breite Signatur im IR-Spektrum. ${ }^{158}$ Mit einer Linienbreite von maximal $6.7 \mathrm{~cm}^{-1}$ würde der IR-Laser immer nur einen kleinen Anteil an Monomeren anregen. Außerdem ist das Methanol-Monomer nicht nur in der Expansion vorhanden, sondern auch im Hintergrundgas, wodurch es bereits auf der Gesamtstrecke des Laserstrahls durch die Kammer zu einer Abschwächung der Intensität kommt. Bei einem Molekülaggregat kommt es nur zu einer geringen Abschwächung vor der Wechselwirkungszone mit dem Raman-Laser, da das Aggregat nur in der Überschallexpansion stabil ist und danach wieder zerfällt. Dementsprechend wurde als Ausgangspunkt das kleinste Methanolaggregat, das Dimer, gewählt. Die Struktur des Dimers ist bereits seit Anfang der 1990er Jahre bekannt. ${ }^{159}$ Neben zahlreichen IR-Photodissoziationsstudien von Methanolclustern ${ }^{1,159}$ wurde das Dimer auch mittels Mikrowellenspektroskopie, ${ }^{23,160}$ cavity-ringdown-Spektroskopie, ${ }^{24}$ Matrixisolationsspektroskopie ${ }^{25,161}$ sowie durch FTIR- und Raman-Spektroskopie in Überschallexpansionen ${ }^{12}$ charakterisiert. Die Position der OH-Streckschwingungsbande des Wasserstoffbrückendonormoleküls wurde zu $3575.4 \mathrm{~cm}^{-1}$ bestimmt, ${ }^{12,24}$ hingegen existieren für die Position der OH-Streckschwingung des Akzeptors verschiedene experimentell bestimmte Werte von $3683.8 \mathrm{~cm}^{-124}$ und $3675 \mathrm{~cm}^{-1}$. ${ }^{162}$ Für die Dissoziationsenergie wurde zunächst von Bizzarri et al. eine obere Grenze von ca. $14 \mathrm{~kJ} / \mathrm{mol}$ bestimmt ${ }^{163}$ und später wurde die Dissoziationsenergie von Huisken et al. auf $<40 \mathrm{~kJ} / \mathrm{mol}$ eingegrenzt. ${ }^{159}$ Eine neue theoretische Studie zeigt, dass die Untergrenze für die Dissoziationsenergie bei $18 \mathrm{~kJ} / \mathrm{mol}$ liegt. ${ }^{164}$ In jedem Fall liegt die Anregungsenergie des IR-Laserphotons von $3575 \mathrm{~cm}^{-1}$ deutlich über den genannten Werten, so dass eine Dissoziation des Dimers nach Anregung sehr wahrscheinlich ist. In Analogie zu vergleichbaren Dimeren wie $(\mathrm{HF})_{2}$ ist damit zu rechnen, dass der Dissoziationsprozess auf einer sub-ps-Zeitskala abgeschlossen ist, für die aktuellen Experimente also in guter Näherung als instantan betrachtet werden kann. ${ }^{165,166}$ 


\subsection{Abschätzung des zu erwartenden Effektes auf das Raman-Spektrum}

Unter der Annahme, dass jede Absorption eines IR-Photons mit der OH-Streckschwingungswellenzahl des Wasserstoffbrückendonormoleküls von $3575 \mathrm{~cm}^{-1}$ zur Dissoziation des Aggregates führt, kann die Stärke des Effektes der IR-Laseranregung abgeschätzt werden. Hierfür muss einerseits die Anzahl an dissoziierten Dimeren im Wechselwirkungsvolumen sowie die Gesamtzahl der Dimere in diesem berechnet werden. Das Verhältnis beider ergibt dann die zu erwartende Abschwächung des Raman-Signals.

Die Gesamtzahl der Methanol-Dimere kann folgendermaßen bestimmt werden:

$$
N_{\text {gesamt }}=c \cdot \sigma \cdot \delta
$$

Hierbei ist $c$ die Konzentration, $\delta$ die Wechselwirkungslänge des IR-Lasers mit der Expansion vor der Raman-Anregung und $\sigma$ der Querschnitt, auf den der Laser über die Wechselwirkungslänge fokussiert ist.

Die Anzahl der dissoziierten Dimere kann über das Lambert-Beer'sche Gesetz berechnet werden. Unter der Annahme, dass die Intensitätsänderung klein gegenüber der Gesamtintensität ist, kann eine linearisierte Form verwendet werden,

$$
I_{0}-I=\kappa \cdot c \cdot \delta \cdot I_{0}
$$

mit $\kappa$ als molarem Neperschem Absorptionskoeffizienten und $I_{0}$ als Anfangsintensität. Aus der Leistung des IR-Lasers und der Wellenzahl, bei der er emittiert, kann der anfängliche Photonenfluss $\phi_{0}$ bestimmt werden. Er wird anstelle der Intensität eingesetzt. Hierdurch ergeben sich dann die pro Sekunde dissoziierten Dimere $\dot{N}_{\text {diss }}$.

$$
\dot{N}_{\text {diss }}=\phi_{0}-\phi=\kappa \cdot c \cdot \delta \cdot \phi_{0}
$$

Der Absorptionskoeffizient wird aus der integrierten Bandenstärke $S$ sowie der Breite der Absorptionsbande $\Delta$ bestimmt. Vereinfachend wird hierbei ein Rechteckprofil angenommen. Somit ergibt sich als Absorptionskoeffizient:

$$
\kappa=S / \Delta
$$

Um nun aus der Dissoziationsrate $\dot{N}_{\text {diss }}$ die Anzahl der auf der Wechselwirkungsstrecke dissoziierten Dimere zu bestimmen, wird die Fluggeschwindigkeit $v$ der Aggregate einbezogen. Der Quotient aus der Wechselwirkungslänge $\delta$ und der Fluggeschwindigkeit $v$ gibt an, in welcher Zeit die Wechselwirkungslänge durchquert wird, und durch 
Multiplikation mit der Dissoziationsrate ergibt sich somit die Anzahl der auf der Wechselwirkungslänge dissoziierten Dimere, so lange diese gegenüber der Gesamtzahl $N_{\text {gesamt }}$ klein ist.

$$
N_{\text {diss }}=\frac{S}{\Delta} \cdot c \cdot \delta \cdot \phi_{0} \cdot \frac{\delta}{v}
$$

Die Abschwächung des Raman-Signals $A$ ergibt sich dann aus dem Verhältnis der Anzahl der dissoziierten und der gesamten Dimere im Wechselwirkungsvolumen, so lange dieses Verhältnis klein ist:

$$
\begin{aligned}
A & =\frac{N_{\text {diss }}}{N_{\text {gesamt }}}=\frac{\frac{S}{\Delta} \cdot c \cdot \delta \cdot \phi_{0} \cdot \frac{\delta}{v}}{c \cdot \sigma \cdot \delta} \\
& =\frac{S \cdot \delta \cdot \phi_{0}}{\Delta \cdot \sigma \cdot v}
\end{aligned}
$$

Eine beispielhafte Abschätzung mit folgenden Werten für die gegebenen Größen:

Photonenfluss $\phi_{0}$ bei $5 \mathrm{~W}$ und $3575 \mathrm{~cm}^{-1}: 7 \cdot 10^{19} \mathrm{~s}^{-1}$

Bandenstärke $S:{ }^{a}$

Wechselwirkungslänge $\delta$ :

Bandenbreite $\Delta$ :

Querschnitt $\sigma$ :

Fluggeschwindigkeit $v:^{27}$
$461 \mathrm{~km} \mathrm{~mol}^{-1}$

$=7.7 \cdot 10^{-17} \mathrm{~cm}$

$0.1 \mathrm{~cm}$

$5 \mathrm{~cm}^{-1}$

$0.01 \cdot 0.2 \mathrm{~cm}^{2}$

$1770 \mathrm{~m} / \mathrm{s}$

$=177000 \mathrm{~cm} / \mathrm{s}$

${ }^{a}$ Aus Ref. 164 (ESI); harmonisch berechnet auf LMP2/A'VQZ-Niveau.

ergibt eine Abschwächung von $30 \%$.

Es kommt also gemäß dieser groben Abschätzung zu einer signifikanten Abschwächung. Bei der angegebenen Fluggeschwindigkeit wird von Helium als Trägergas ausgegangen. Bei Verwendung von Argon kann gemäß dieser Abschätzung der zu erwartende Effekt um mehr als Faktor 3 auf eine Abschwächung von $96 \%$ verstärkt werden, wobei hier allerdings die oben gewählte lineare Form nicht mehr gültig ist.

Die Dimerkonzentration $c$ fällt bei dieser Abschätzung heraus. Sie ist jedoch notwendig um die relative Abschwächung des IR-Lasers abzuschätzen. Diese ergibt sich als $\frac{\left(\phi_{0}-\phi\right)}{\phi_{0}}=\frac{S}{\Delta} \cdot c \cdot \delta$. Für die Dimerkonzentration kann in Übereinstimmung mit den Referenzen 167 und 24 eine Größenordnung von $10^{13} \mathrm{~cm}^{-3}$ angenommen werden. Hieraus ergibt sich dann mit den oben genannten Werten für die anderen Parameter ein Wert von $1.54 \cdot 10^{-5}$. Es kommt also zu einer sehr geringen Abschwächung der IR-Laserleistung, 
so dass die angenommene Näherung für das Lambert-Beer-Gesetz gerechtfertigt ist. Die Anzahl der vom OPO-Laser emittierten Photonen ist dementsprechend mehr als ausreichend um den erwarteten Effekt zu erzielen.

\subsection{Testmessungen unter Nutzung der Paraboldüse}

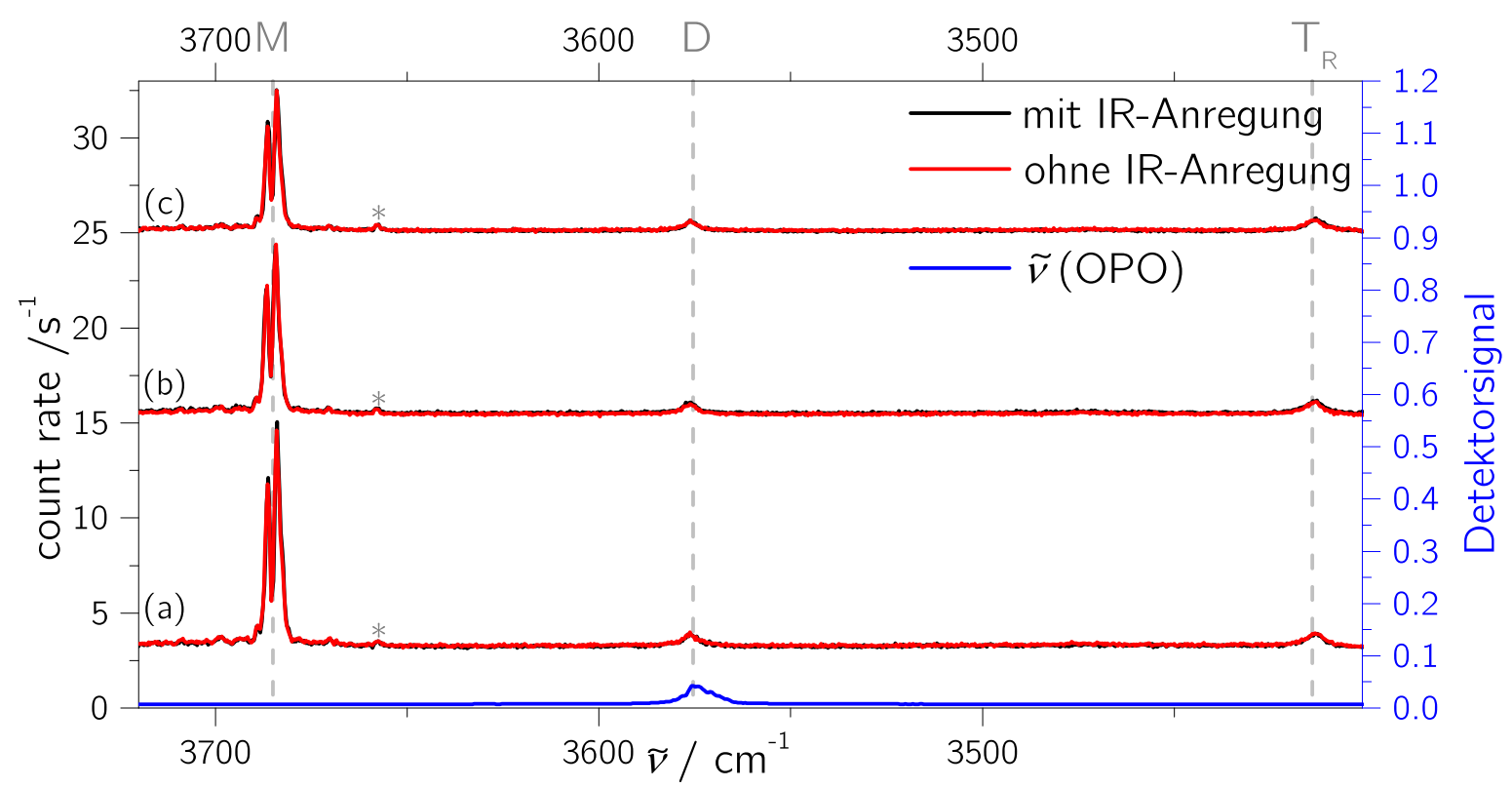

Abbildung 7.1: Raman-Jetspektren von Methanol in Helium. Aufgenommen bei (a) $2.5 \mathrm{~mm}$, (b) $3.0 \mathrm{~mm}$ und (c) $3.5 \mathrm{~mm}$ Abstand zwischen Raman-Laser und Düse. In rot sind jeweils Spektren gezeigt, die inklusive IR-Anregung aufgenommen wurden, dahinter in schwarz ohne IR-Anregung. In blau ist außerdem das FTIR-Spektrum des OPO-Lasers gezeigt. Es sind die Raman-Signale von Monomer (M), Dimer (Donor) (D) und Trimer $\left(T_{R}\right)$ zu erkennen. Mit * ist die symmetrische Streckschwingungsbande von Wasser, das als Verunreinigung sowohl aus Methanol selbst als auch aus den Zuleitungen in die Expansion gelangt sein kann, gekennzeichnet. Angaben über die Messbedingungen finden sich im Anhang A.3.

Für die ersten Testmessungen wurde zunächst Helium als Trägergas gewählt. Die Sättigertemperatur wurde möglichst kalt gewählt $\left(-25^{\circ} \mathrm{C}\right)$ um zu verhindern, dass sich signifikante Anteile an größeren Clustern $(n>3)$ in der Expansion befinden. In Abbildung 7.1 sind drei Spektrenpaare gezeigt, die bei verschiedenen Abständen der Paraboldüse zum Raman-Laser aufgenommen wurden. Jeweils in schwarz sind Spektren ohne eine IR-Anregung und rot Spektren mit IR-Anregung gezeigt. In blau ist das zugehörige FTIR-Spektrum des OPO-Lasers gezeigt mit der zugehörigen rechten $y$-Achse. 
Dieser Farbcode wurde auch in den folgenden Abbildungen verwendet. Es wurden jeweils hintereinander ein Spektrum mit und eines ohne IR-Laseranregung aufgenommen. Abbildung 7.1 zeigt um $3685 \mathrm{~cm}^{-1}$ die beiden Monomersignale (M), die durch Tunnelaufspaltung entstehen, bei $3576 \mathrm{~cm}^{-1}$ das Dimersignal (D) (des Donormoleküls) und bei $3413 \mathrm{~cm}^{-1}$ das Raman-Signal des cyclischen Trimers $\left(T_{R}\right)$ sowie außerdem eine geringe Menge Wasser $\left(^{*}\right)$, die anhand des Signals der symmetrischen Streckschwingungsbande bei $3657 \mathrm{~cm}^{-1}$ im Spektrum zu erkennen ist. ${ }^{12}$ Die Monomersignale sinken mit steigendem Abstand zwischen Düse und Raman-Anregungslaser bedingt durch den Dichteabfall während der Expansion. Außerdem ist zu erkennen, dass sich weder die relative noch die absolute Signalstärke der beiden Monomerbanden verändert, wenn zusätzlich der IR-Laser eingekoppelt wird. Die IR-Anregung scheint also keinen signifikanten Einfluss auf die Monomerbanden zu haben. Außerdem zeigt sich hierdurch, dass die Expansionsbedingungen sehr stabil sind und somit die Spektren gut vergleichbar sind.

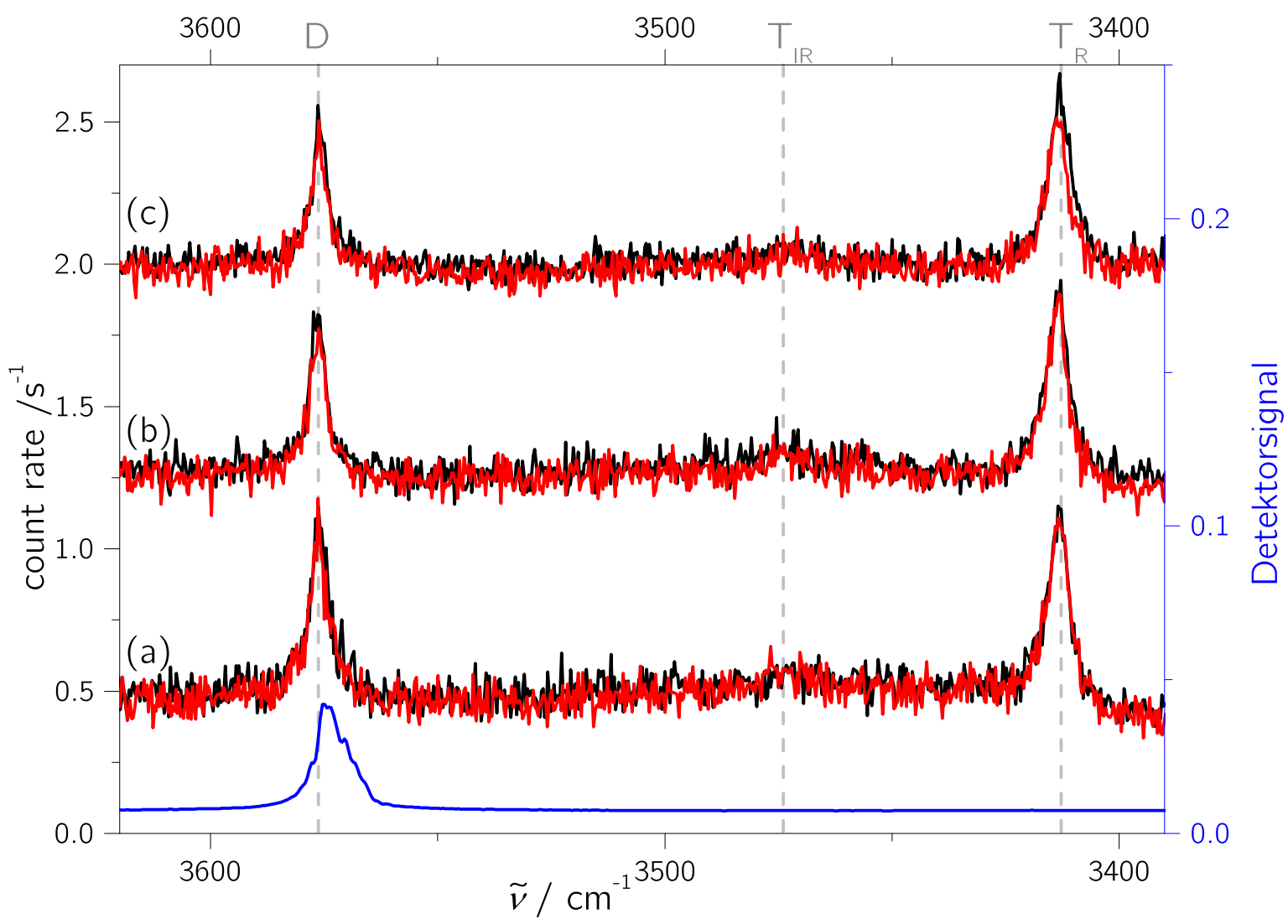

Abbildung 7.2: Vergrößerter Ausschnitt aus Abb. 7.1. Die Spektren sind unskaliert. Neben den schon zugeordneten, stark Raman-aktiven Dimer- $(D)$ und Trimerpeaks $\left(T_{R}\right)$ ist auch noch eine schwach Raman-aktive Bande $\left(\mathrm{T}_{\mathrm{IR}}\right)$ zu erkennen. Bei einem Abstand von $3.5 \mathrm{~mm}$ (c) zeigt sich eine geringe Signalabnahme bei dem Trimerpeak $\left(\mathrm{T}_{\mathrm{R}}\right)$ nach IR-Anregung des Dimers. 
Zur genaueren Analyse der Auswirkungen der IR-Anregung auf Dimer- und Trimersignale ist in Abbildung 7.2 ein vergrößerter Ausschnitt aus Abbildung 7.1 gezeigt. Neben den schon beschriebenen Dimer und Trimer-Signalen ( $D$ und $T_{R}$ ) ist außerdem noch ein Signal bei $3475 \mathrm{~cm}^{-1}$ zu erkennen, welches einer stark IR-aktiven und nur sehr schwach Raman-aktiven Trimerbande zugeordnet wurde $\left(\mathrm{T}_{\mathrm{IR}}\right)$ in Übereinstimmung mit Daten aus Ref. 12 .

Die Signalstärke des Dimerpeaks sinkt um wenige Prozent von Spektrenpaar (a) nach (c) ab, während die des starken Trimerpeaks zunimmt. Effekte aufgrund von IRLaseranregung sind minimal in Spektrenpaar (b) beim Dimer und bei Spektrenpaar (c) etwas deutlicher beim Trimer als Signalabnahme zu erkennen.

Die beobachteten Effekte sind sehr gering und statistisch kaum signifikant. Dies kann darauf zurückgeführt werden, dass die Fokuseigenschaften der Düse nicht ideal sind. Zudem weist das Auftreten eines Effektes beim Trimer, obwohl die Wellenzahl des IR-Lasers auf das Dimer eingestellt wurde, darauf hin, dass es sich um einen rein thermischen Effekt handelt. Der IR-Laser heizt lediglich die Düse auf, wodurch die Bildung von Trimeren teilweise unterdrückt wird.

Aufgrund dieses Befundes wurde der Ansatz der Düse mit Parabolrinne nicht weiterverfolgt, sondern die schon in Kapitel 3.3.5 erwähnte Zylinderlinse verwendet. 


\subsection{Testmessungen unter Nutzung der Zylinderlinse}

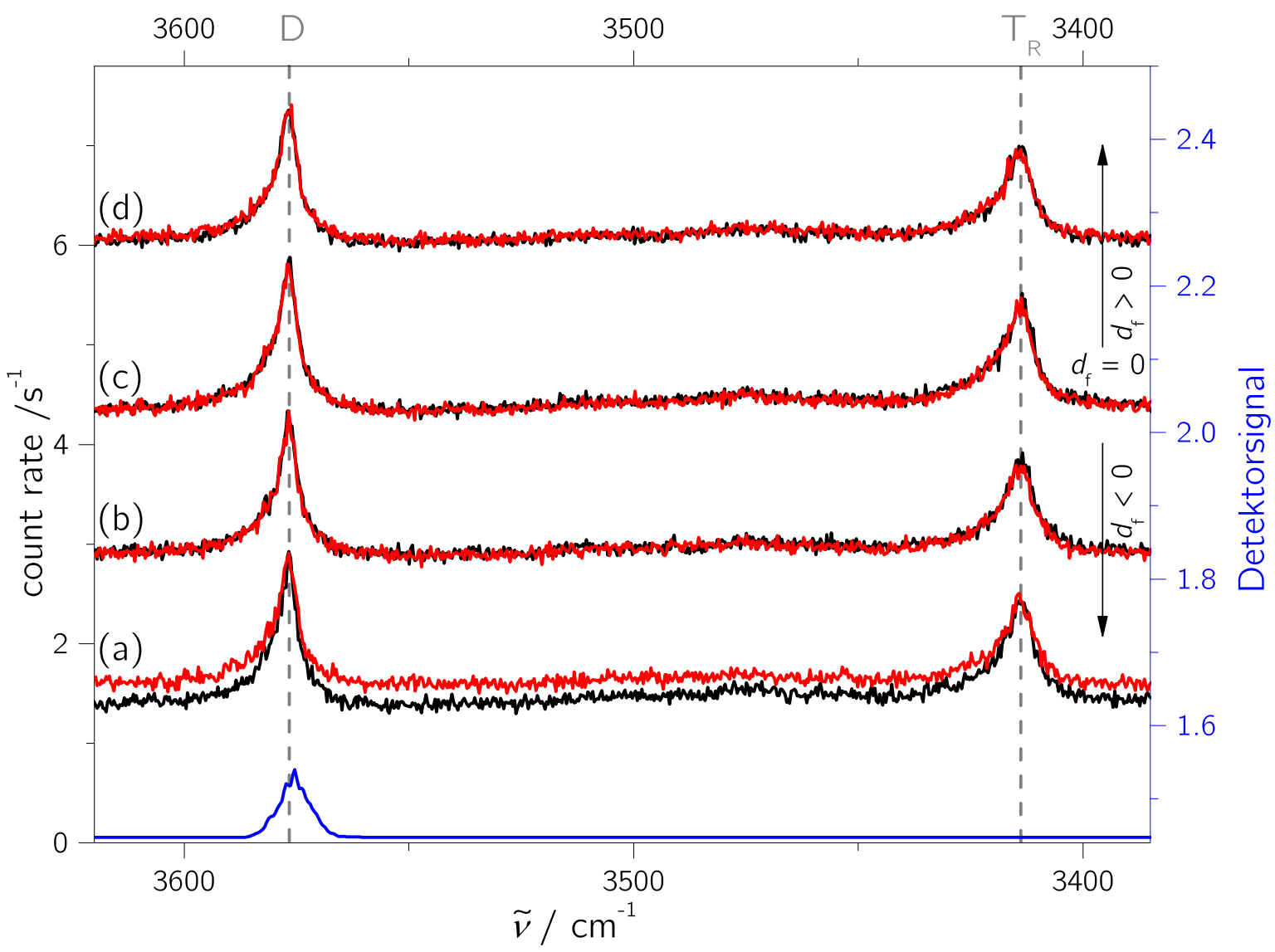

Abbildung 7.3: Signaländerung durch IR-Anregung in Abhängigkeit vom Abstand der Brennpunkte $d_{\mathrm{f}}$ von Zylinder- und Raman-Laserlinse. (a) $d_{\mathrm{f}}=-1 \mathrm{~mm}$ (IR-Fokus liegt auf der Düsenspitze), (b) $d_{\mathrm{f}}=-0.5 \mathrm{~mm}$ (IR-Anregung geschieht vor Raman-Anregung), (c) $d_{\mathrm{f}}=0 \mathrm{~mm}$ (Brennlinien überlappen), (d) $d_{\mathrm{f}}=0.5 \mathrm{~mm}$ (IR-Fokus liegt hinter der Raman-Anregung). Aufnahmeparameter: $p_{\mathrm{s}}=0.7 \mathrm{mbar}, 6 \times 100 \mathrm{~s}, d=1 \mathrm{~mm}$, Trägergas Helium. Weitere Angaben über die Messbedingungen finden sich in Anhang A.3.

Bei Verwendung der $\mathrm{CaF}_{2}$-Zylinderlinse wurde zunächst ebenfalls Helium als Trägergas verwendet. Ein erster Test bestand darin, die Signaländerung in Abhängigkeit von der relativen Position der (näherungsweise) Brennlinien von Raman-Laserlinse und Zylinderlinse zu untersuchen. Abbildung 7.3 zeigt vier Spektrenpaare, die nach zunehmendem Abstand der Brennlinien geordnet sind. Bei Spektrenpaar (a) liegt der Fokus der IR-Strahlung direkt auf der Düse, bei (b) $0.5 \mathrm{~mm}$, bei (c) $1 \mathrm{~mm}$ und bei (d) $1.5 \mathrm{~mm}$ vor der Düse. Spektrum (c) ist somit dasjenige Spektrum, bei dem die beiden Brennlinien überlappen sollten, da ein Abstand von $1 \mathrm{~mm}$ zwischen Düse und Raman-Anregungslaser gewählt wurde. Bei Spektrum (a) zeigt sich eine Erhöhung der 
Basislinie, die mit einem stärkeren Leuchten der Düse korreliert ist. Auffällig ist allerdings, dass die Peakhöhe von Dimer und Trimer relativ zur Basislinie abnimmt, was wahrscheinlich auf das Aufheizen der Düse zurückzuführen ist. In den anderen Fällen kann kein signifikanter Effekt ausgemacht werden. Dieser Befund gab den Anlass das Trägergas von Helium auf Argon umzustellen, da als Hauptgrund für das Ausbleiben eines Effektes die große Fluggeschwindigkeit vermutet wurde, die dazu führt, dass die Wechselwirkungszeit mit dem Laser zu kurz ist um zu einer Dissoziation zu führen.

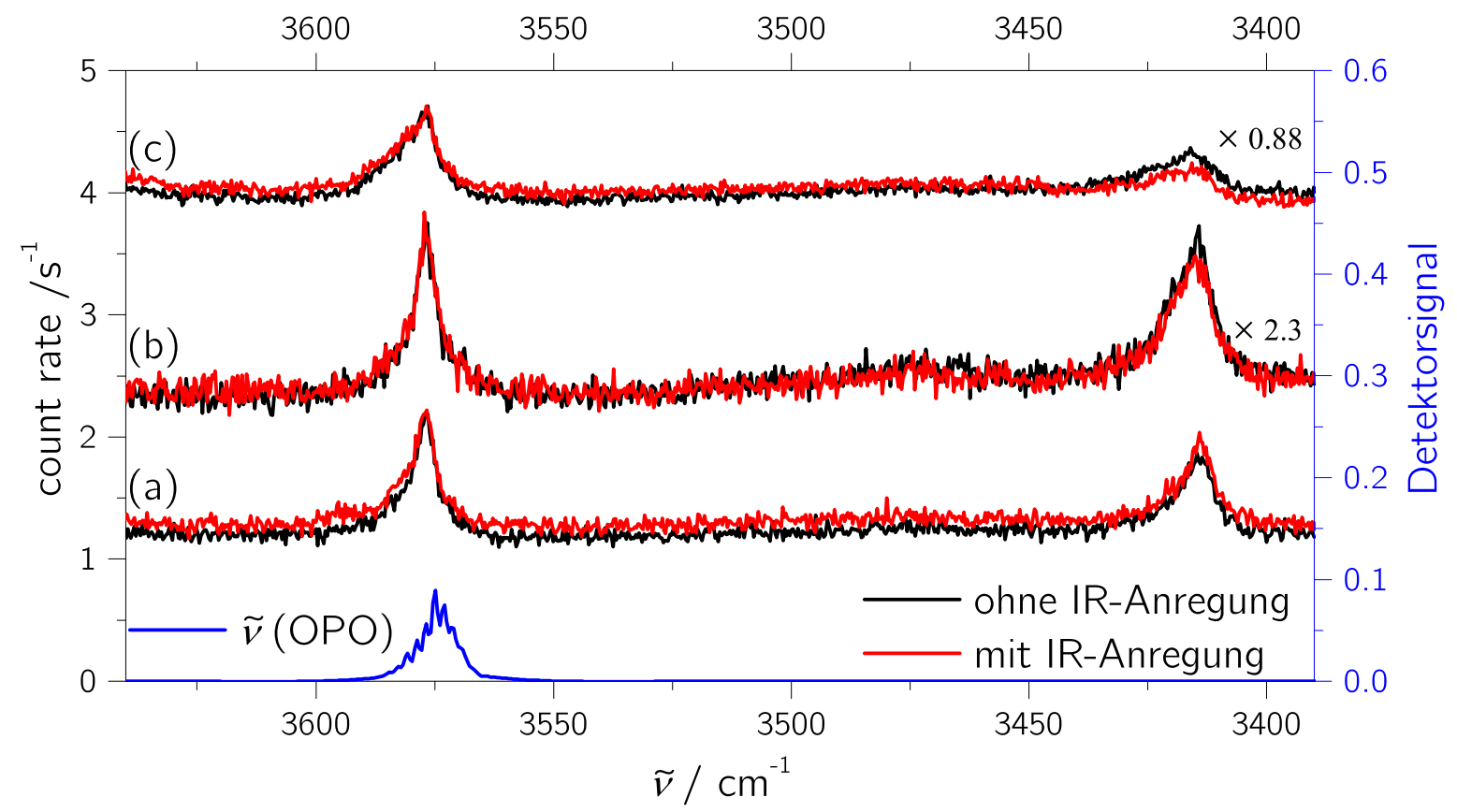

Abbildung 7.4: Vergleich von Expansionen mit Helium (a) und Argon (b, c) als Trägergas. Expansionsbedingungen: (a) $p_{\mathrm{s}}=0.7 \mathrm{mbar}, 6 \times 100 \mathrm{~s}, d=1 \mathrm{~mm}$, (b) $p_{\mathrm{s}}=0.5 \mathrm{mbar}$, $12 \times 100 \mathrm{~s}, d=1 \mathrm{~mm}$, Skalierungsfaktor: 2.3 ; (c) $p_{\mathrm{s}}=0.5 \mathrm{mbar}, 12 \times 100 \mathrm{~s}, d=0.4 \mathrm{~mm}$, Skalierungsfaktor: 0.88, OPO-Wellenzahl: $3575 \mathrm{~cm}^{-1}$. Weitere Angaben über die Messbedingungen finden sich im Anhang A.3.

In Abbildung 7.4 ist ein Vergleich einer Expansion von Methanol in Helium (Spektren (a)) mit zwei verschiedenen Expansionen in Argon (Spektren (b) und (c)) gezeigt, wobei die Brennlinien beider Laser bei IR-Anregung überlappen. Die Spektren wurden auf gleiche Monomerpeakhöhe skaliert, wobei Spektrenpaar (a) als Referenz diente. Die Spektrenpaare (a) und (b) sind jeweils im Abstand von $1 \mathrm{~mm}$ zur Düse aufgenommen worden. Es zeigt sich, dass durch die Verwendung des schwereren Trägergases der Dimer- und Trimeranteil relativ zum Monomer deutlich erhöht ist. Allerdings ist aufgrund der stärkeren Rayleighstreuung das Signal-zu-Rausch-Verhältnis schlechter, was auch durch eine doppelt so lange Messzeit nicht ausgeglichen werden konnte. Bei IR-Anregung zeigt sich für das Spektrenpaar (a) eine leichte Erhöhung der Basislinie, die auch hier auf das Leuchten der Düse zurückzuführen ist. Beim Spektrenpaar (b) 
scheint das Leuchten nur sehr gering zu sein. Außerdem ist eine geringfügige Signalabnahme beim Trimerpeak zu erkennen. Bei geringerem Abstand von $0.4 \mathrm{~mm}$ zur Düse (Spektrum (c)) wird diese Signalabnahme sehr viel deutlicher. Außerdem wird das Dimersignal breiter, was darauf zurückgeführt werden kann, dass das System noch nicht vollständig relaxiert ist und somit vermehrt intermolekulare Schwingungsfreiheitsgrade populiert sind.

Ein gravierendes Problem für die Auswertung der Spektren stellt das Leuchten der Düse dar. In Abbildung A.8.1 im Anhang sind drei Spektrenpaare gezeigt, bei denen die Justierung unterschiedlich gut gelang und somit die Unterdrückung des Leuchtens unterschiedlich wirksam erfolgte. Bei besonders guter Justierung erfolgt eine Abnahme der Basislinienhöhe, während bei besonders schlechter Justierung die Basislinie so stark ansteigt, dass durch das Leuchten die Peaks überstrahlt werden und daher eine Auswertung nicht möglich ist. Die Abnahme der Basislinie kann auch auf das Aufheizen der Düse zurückgeführt werden, da hierdurch größere Cluster, insbesondere Argonumhüllte Spezies, die besonders starke Rayleighstreuer sind, in geringerem Umfang gebildet werden. Entsprechend konnten ausschließlich Spektren sinnvoll ausgewertet werden, bei denen es lediglich zu einer geringen Erhöhung der Basislinie kommt.

Die in Abbildung 7.4 (c) beobachtete Signalabnahme im Trimer ist bei Einstrahlung des IR-Lichts bei der Dimerwellenzahl vermutlich auf ein Aufheizen der Düse zurückzuführen. Als nächster Schritt wurde daher die Signaländerung in Abhängigkeit von der OPO-Wellenzahl untersucht. In Abbildung 7.5 sind drei Spektrenpaare gezeigt, bei denen die OPO-Wellenzahl (a) auf die Absorptionsbande des Dimers, (b) zwischen Absorptionsbanden und (c) auf die starke IR-Absorptionsbande des Trimers eingestellt wurde.

Die Ausgangsleistung des OPO-Lasers war mit ca. $6 \mathrm{~W}$ konstant. Anhand der Basislinienänderung ist zu erkennen, dass das Düsenleuchten in allen drei Fällen relativ effizient unterdrückt wurde, so dass die Spektren gut miteinander verglichen werden können.

In Spektrum (a) zeigt sich eine geringe Erniedrigung des Trimersignals, in (b) eine vergleichsweise große Erniedrigung bei Dimer und Trimer und bei (c) eine geringe Erniedrigung des Dimersignals und eine größere beim Trimerpeak. Auffällig ist besonders, dass der stärkste Effekt bei einer Einstrahlung in einem Bereich, in dem keine starke Absorptionsbande vorhanden ist, erzielt wird.

Die Effekte sind auch bei Nutzung der Zylinderlinse zu unspezifisch, als dass sie allein auf die IR-Anregung eines Aggregates zurückzuführen sind. Vielmehr scheint das Aufheizen der Düse durch den IR-Laser für die Signaländerungen verantwortlich zu sein. Für eine Quantifizierung dieses lokalen Heizens wurden Spektren unter Nutzung der Düsenheizung aufgenommen. Abbildung 7.6 zeigt den Vergleich von Spektrenpaar (b) aus Abbildumg 7.5 mit drei Spektren, die bei Düsentemperaturen von $25^{\circ} \mathrm{C}$ (schwarz), $80^{\circ} \mathrm{C}$ (dunkelgrau) und $150^{\circ} \mathrm{C}$ (hellgrau) aufgenommen wurden. Insgesamt zeigt sich, dass das Verhalten der Banden bei Nutzung des IR-Lasers ähnlich ist wie bei Nutzung 


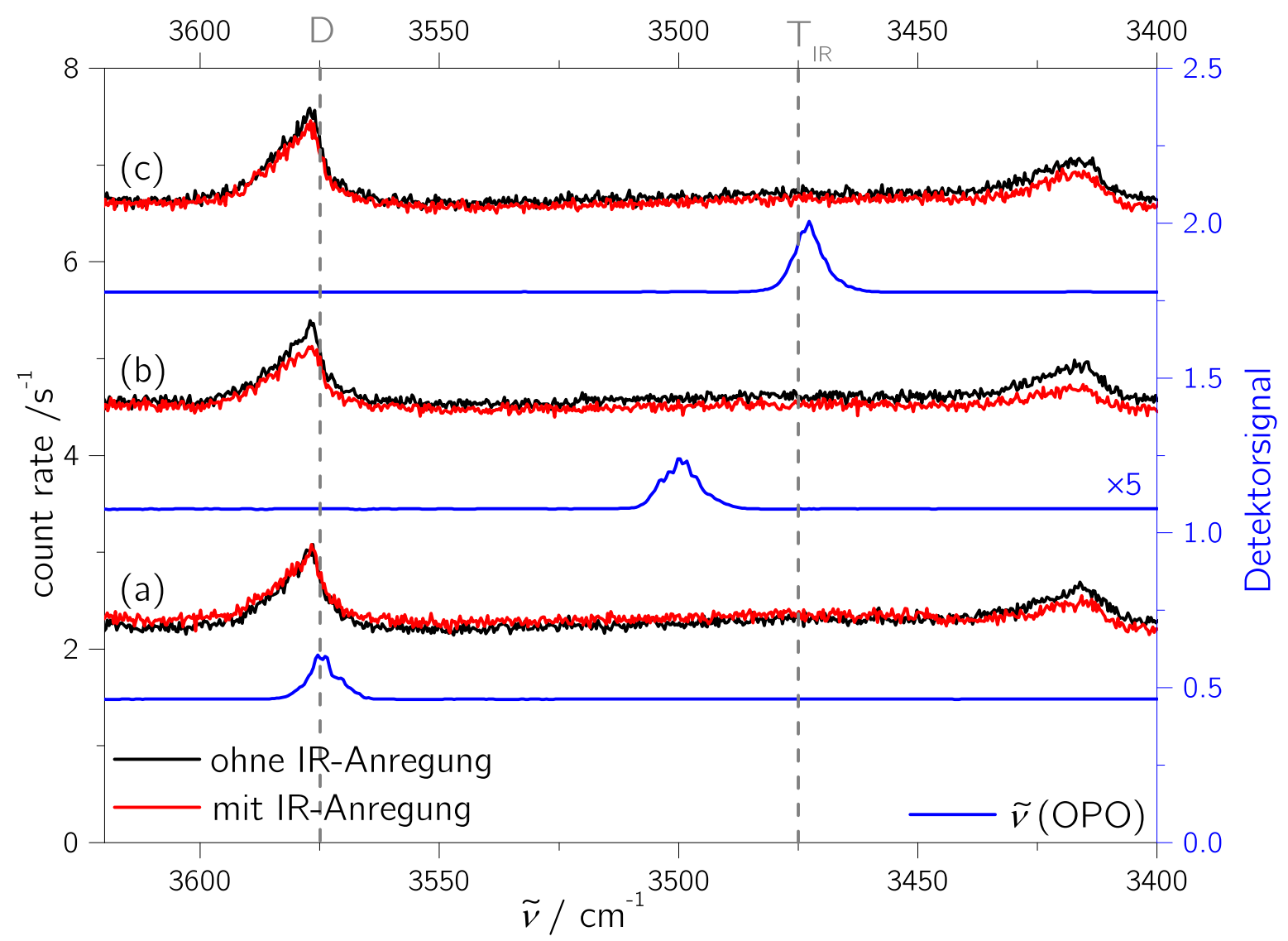

Abbildung 7.5: Vergleich der Signaländerung von Dimer und Trimer bei IR-Anregung mit verschiedenen OPO-Wellenzahlen. (a) $3575 \mathrm{~cm}^{-1}$ (Dimerabsorption), (b) $3500 \mathrm{~cm}^{-1}$ (keine starke Absorptionsbande) und (c) bei $3474 \mathrm{~cm}^{-1}$ (starke IR-Absorptionsbande des Trimers $)^{12}$ mit jeweils Argon als Trägergas und einem Abstand zur Düse von $0.4 \mathrm{~mm}$. Weitere Angaben über die Messbedingungen finden sich in Anhang A.3. 


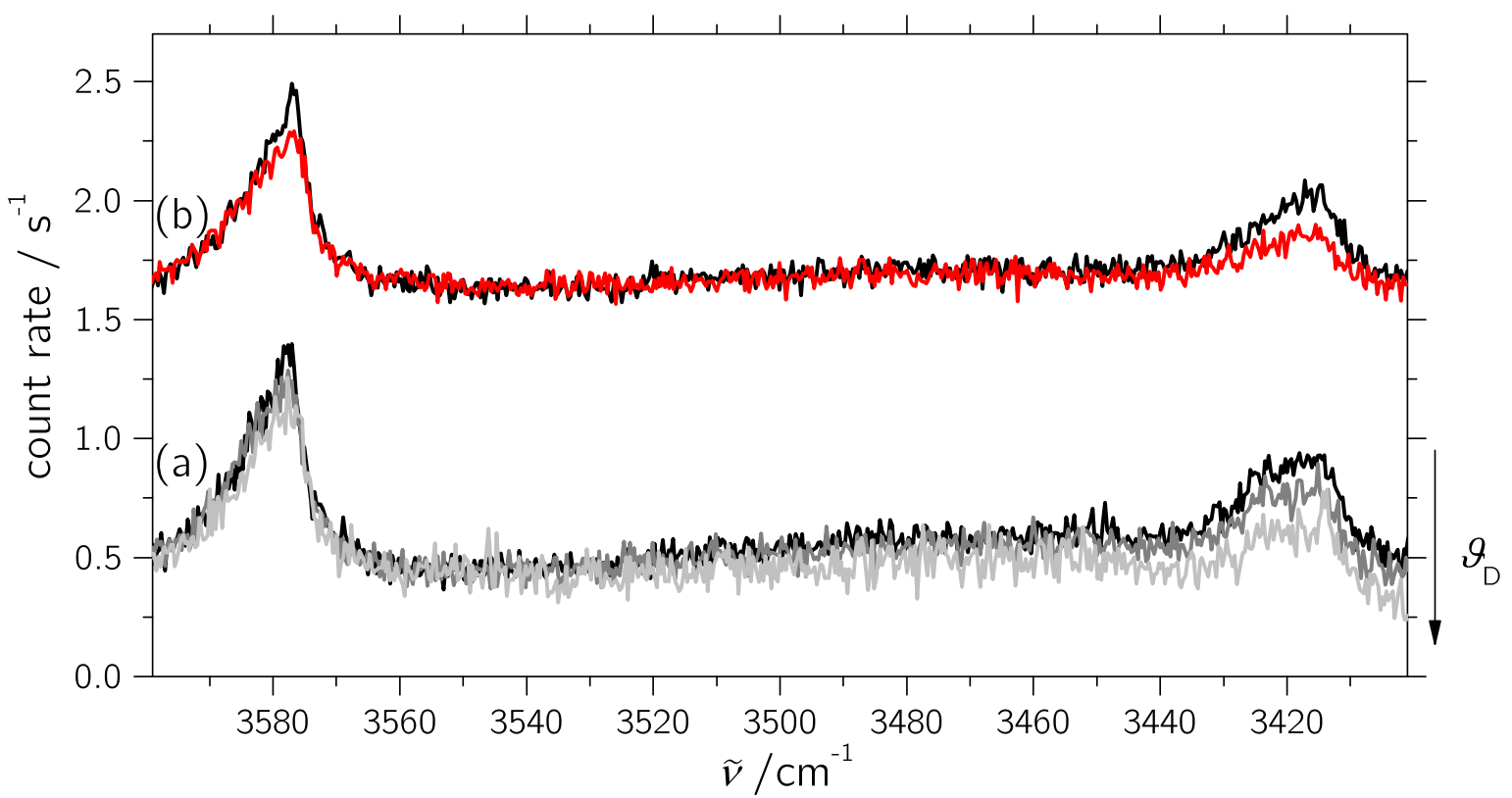

Abbildung 7.6: Vergleich von Spektrenpaar (b) aus Abbildung 7.5 (b) mit Spektren (a), die bei verschiedenen Düsentemperaturen aufgenommen wurden. (schwarz) $\vartheta_{\mathrm{D}}=25^{\circ} \mathrm{C}$, (dunkelgrau) $\vartheta_{\mathrm{D}}=80^{\circ} \mathrm{C}$ und (hellgrau) $\vartheta_{\mathrm{D}}=150^{\circ} \mathrm{C}$. Expansionsbedingungen jeweils: Trägergas Argon, $p_{\mathrm{s}}=0.5 \mathrm{mbar}, 12 \times 100 \mathrm{~s}, d=0.4 \mathrm{~mm}$. Weitere Angaben über die Messbedingungen finden sich im Anhang A.3.

der Düsenheizung bei hoher Temperatur. Hierdurch wird bestätigt, dass der Großteil der beobachteten Signalabnahme auf die lokale Heizung der Düse zurückzuführen ist. Das mit IR-Anregung aufgenommene Spektrum ist dem hellgrauen Spektrum am ähnlichsten. Der lokale Heizeffekt durch den OPO-Laser hat also eine ähnliche Auswirkung wie das Aufheizen der gesamten Düse auf $150^{\circ} \mathrm{C}$.

\subsection{Diskussion und Ausblick}

Die Diskrepanz zwischen der in Kapitel 7.1 abgeschätzten Abschwächung und der experimentell beobachteten Signaländerung ist auf verschiedene Probleme zurückzuführen.

Das Hauptproblem der Abschätzung besteht darin, dass angenommen wird, dass die gesamte Laserleistung des OPO genutzt werden kann. Es wird also davon ausgegangen, dass der OPO-Laser idealerweise auf die gleiche Breite wie der Raman-Anregungslaser fokussiert ist.

Die tatsächliche Überlappung der beiden Laser ist allerdings nicht ideal. Um die maximale Überlappung zu bestimmen müssen die Strahlradien $\omega_{0}^{\prime}$ der beiden Laserstrahlen 


\begin{tabular}{ccccc}
\hline Laser & $\lambda / \mathrm{nm}$ & $\omega_{0} / \mathrm{mm}$ & $f / \mathrm{mm}$ & $\omega_{0}^{\prime} / \mu \mathrm{m}$ \\
\hline Verdi V18 & 532 & 1.125 & 50 & 7.5 \\
Argos OPO & 2790 & 2.1 & 67 & 29 \\
\hline
\end{tabular}

Tabelle 7.1: Wellenlänge $\lambda$, Strahlradius vor Fokussierung $\left(\omega_{0}\right)$ und im Fokus $\left(\omega_{0}^{\prime}\right)$, sowie Brennweite der jeweiligen Linse $f$ für den Raman-Laser (Verdi V18) und den IR-Laser (Argos OPO).

im Fokuspunkt bestimmt werden. Unter Annahme eines idealen Gaußstrahls kann der Strahlradius $\omega_{0}^{\prime}$ über folgende Formel ${ }^{44}$ bestimmt werden:

$$
\omega_{0}^{\prime}=\frac{\lambda f}{\pi \omega_{0}}
$$

mit $\lambda$ der Wellenlänge des Lasers, $f$ der Brennweite der Linse und $\omega_{0}$ dem Strahlradius vor Passieren der Linse. In Tabelle 7.1 sind die verwendeten Werte angegeben. Für den OPO-Laser wurde eine Wellenzahl von $3575 \mathrm{~cm}^{-1}$ (entsprechend einer Wellenlänge von $2.79 \mathrm{\mu m}$ ) verwendet, also das Absorptionsmaximum des gebundenen $\mathrm{OH}-$ Oszillators des Methanol-Dimers. Die in Tabelle 7.1 angegebenen Werte zeigen, dass auch bei perfekter Justierung maximal 26\% der OPO-Leistung genutzt werden können.

Außerdem nicht einbezogen in die Abschätzung ist, dass der CCD-Chip nicht die gesamte Höhe von 3-4 mm des IR-Lasers aufnimmt, sondern nur $1.37 \mathrm{~mm} .{ }^{9}$ Es gehen hier also schon ca. $50 \%$ des Effektes verloren. Abhilfe schaffen könnte entweder die Nutzung einer zweiten Zylinderlinse mit sehr schwacher Fokussierung, die senkrecht zur ersten Linse fokussiert, oder aber eine Paraboldüse mit einem elliptischen Profil.

Zusammen mit der oben berechneten nutzbaren Leistung von von $26 \%$ ergibt sich also für Helium eine Abschwächung von lediglich 4\% und für Argon von 12\%. Diese wird allerdings überlagert von dem vermutlich stärkeren thermischen Effekt.

Die Abschätzung geht weiterhin davon aus, dass die Dissoziation ausschließlich in der Fokuslinie des IR-Lasers erfolgt. Bei der verwendeten Einkopplungsmethode trifft die Laserstrahlung jedoch bereits zwischen Düsenausgang und der Position des RamanAnregungslasers auf Molekülaggregate und kann diese dissoziieren. Hierdurch wird das Wechselwirkungsvolumen erhöht und somit eine Verstärkung des Effektes erzielt. Allerdings kann es nach IR-Anregung auch zu einer Rekombination kommen, da besonders am Anfang der Expansion noch viele Stöße geschehen und so eine schnelle Relaxation noch möglich ist. Weiterhin nicht einbezogen in die Berechnung ist die Lebensdauer des angeregten, aber noch nicht dissoziierten Dimers. Aufgrund der großen Anregungsenergie und der direkten Verknüpfung der angeregten Schwingung mit der Dissoziationskoordinate ist die Näherung einer direkten Dissoziation jedoch gerechtfertigt. ${ }^{165,166}$

Zur Verstärkung des beobachtbaren Effektes können mehrere Ansätze verfolgt werden. 
Eine bessere Überlappung könnte durch den Einsatz einer weniger stark fokussierenden Raman-Laserlinse und einer stärker fokussierenden Zylinderlinse erzielt werden. Beispielsweise kann durch Verwendung einer Raman-Laserlinse mit $100 \mathrm{~mm}$ Brennweite und entsprechender Anpassung des Monochromatorspaltes eine Verbesserung des Überlapps um den Faktor zwei bei nahezu gleichbleibender Raman-Signalstärke und nur geringfügig schlechterer Auflösung erreicht werden.

Ein anderer Ansatz besteht darin das Signal-zu-Rausch-Verhältnis der Raman-Messung zu verbessern, denn so können besonders schwache Effekte besser beobachtet werden. Hierfür soll ein stärkerer Raman-Anregungslaser mit einer Maximalleistung von $25 \mathrm{~W}$ erworben werden. Diese Maßnahme ist schon allein deswegen notwendig, da der in dieser Arbeit verwendete Laser derzeit nicht mehr funktionsfähig ist, somit vorerst generell keine Möglichkeit besteht Raman-Messungen mit ähnlichem S/N-Verhältnis an der Curry-Jet Apparatur durchzuführen.

Durch das bessere Signal-zu-Rausch-Verhältnis könnte auch der Messbereich der CCDKamera auf eine geringere Höhe eingestellt werden. Hierfür wird die Monochromatorspalthöhe verringert, so dass nur der mittlere Bereich der Expansion auf die CCDKamera abgebildet wird. Das würde bei Verwendung eines leistungsstärkeren RamanAnregungslasers zu einem ähnlichen S/N-Verhältnis wie in der zuvor verwendeten Anordnung führen. In Kombination mit einer sphärische Linse zur Fokussierung des IRLasers könnte so die nutzbare IR-Leistung deutlich erhöht werden, da der IR-Laser exakt auf den von der CCD-Kamera detektierten Bereich der Expansion fokussiert würde.

Nicht sinnvoll ist hingegen der Einsatz eines leistungsstärkeren OPO-Lasers, da hierdurch die Probleme, die durch das Aufheizen der Düse entstehen, also sowohl das Glühen der Düse als auch die Unterdrückung der Clusterbildung, verstärkt würden.

Des Weiteren kann die maximale Dimerkonzentration zur Verbesserung des S/N-Verhältnisses optimiert werden. Hierzu kann auch auf andere Systeme zurückgegriffen werden. Erste Testmessungen bestehen bereits für das Monomer der Ameisensäure (das Dimer ${ }^{168}$ ist aufgrund der großen Rotverschiebung nicht für den OPO-Laser zugänglich und daher ungeeignet) und das gemischte Methanol-Wasser-Dimer. Da hier allerdings keine Effekte festgestellt werden konnten, sind die Experimente in der vorliegenden Arbeit nicht weiter diskutiert worden.

Methanol ist zwar wie zu Beginn dieses Kapitels beschrieben ein geeigneter Ausgangspunkt, allerdings ist aufgrund der großen Aggregationstendenz die Erzeugung eines hohen relativen Dimeranteils problematisch. Ein Ausweg könnte hier die Nutzung der heizbaren Düse sein, die zur Steuerung der Aggregation eingesetzt würde. So könnten hohe Dimerkonzentrationen trotz hoher Gesamtkonzentration erhalten werden. Durch die Verwendung eines Systems, mit dem - bei ähnlicher Dissoziationsenergie sowie ähnlicher Raman- und IR-Aktivität der zu untersuchenden OH-Streckschwingung - ein 
höherer maximaler Dimeranteil generiert werden kann, kann ein noch besseres Signalzu-Rausch-Verhältnis erhalten werden. Auch hierdurch könnten die erwarteten schwachen Effekte besser beobachtet werden. ${ }^{24,167}$

Als geeignete Systeme sind 2,2,2-Trifluorethanol, tert-Butylalkohol und 1,1,1,3,3,3Hexafluorisopropanol anzusehen. ${ }^{167}$ Die Handhabung von tert-Butylalkohol ist allerdings aufgrund des hohen Schmelzpunktes von $25.3^{\circ} \mathrm{C}^{169}$ schwieriger als bei den anderen beiden Systemen. Messungen von Feststoffen bei Verwendung eines ungeheizten Sättigers zeigen häufig starke Konzentrationsschwankungen. ${ }^{59}$ Für die in diesem Kapitel beschriebenen Messungen ist die Konzentrationsstabilität bei der Expansion jedoch extrem wichtig, da sonst ein Vergleich aufeinander folgender Messungen (mit und ohne IR-Anregung) nicht möglich ist. Zur Messung von tert-Butylalkohol müsste daher der heizbare Sättiger eingesetzt werden, wobei die Düsenheizung auch hier zur Steuerung der Aggregation genutzt würde.

Die beiden Fluoralkohole liegen als Flüssigkeiten vor, so dass die Konzentrationsstabilität gewährleistet ist. Bei 1,1,1,3,3,3-Hexafluorisopropanol ist neben der Dissoziation des Dimers außerdem noch die Untersuchung verschiedener Ar-Umhüllungsstufen, die 2004 zuerst nachgewiesen wurden, ein interessanter Ansatz. ${ }^{59}$ Die Argonummantelung führt allerdings auch zu verstärkter Rayleighstreuung, daher sollte für dieses System zunächst Helium bzw. eine Argon/Helium-Mischung als Trägergas verwendet werden.

Als nächster Schritt ist eine systematische Studie der maximal erreichbaren Dimerkonzentrationen unter Nutzung der Düsenheizung für Methanol, tert-Butylalkohol und die beiden Fluoralkohole sinnvoll, um die optimalen Bedingungen für weitere Testmessungen mit dem OPO-Laser zu bestimmen. Diese Studie ist auch mit einem Raman-Laser geringerer Leistung, wie er zurzeit zur Verfügung steht, möglich. 


\section{Zusammenfassung und Ausblick}

In der vorliegenden Arbeit wurden Raman-Jetspektroskopische Untersuchungen verschiedener Moleküle und Molekülaggregate vorgestellt. Je nach Fragestellung wurde die Curry-Jet Apparatur entweder mit einer heizbaren Düse oder gekoppelt mit einer IR-Laseranregung genutzt. Für die Kopplung mit der IR-Laseranregung wurde der experimentelle Aufbau angepasst. Die Eigenschaften des für die IR-Laseranregung genutzten Dauerstrich-OPO-Lasers wurden vor der Einkopplung in die Curry-Jet Apparatur evaluiert. Durch Einkopplung eines Teils der Laserstrahlung in ein FTIR-Spektrometer konnte eine genaue Wellenlängenbestimmung ermöglicht werden. Außerdem wurden verschiedene experimentelle Ansätze zur Unterdrückung des vom IR-Laser zusätzlich emittierten sichtbaren Lichtes vorgestellt.

Die Temperaturbestimmung aus Stokes- und Anti-Stokes Intensitäten wurde systematisch getestet und als für die Jetspektroskopie geeignet bewertet. Es konnten effektive Rotationstemperaturen von $40-60 \mathrm{~K}$ am Beispiel von Wasser und effektive Schwingungstemperaturen von ca. $100 \mathrm{~K}$ am Beispiel von 2,2,2-Trifluorethanol ermittelt werden. Es zeigten sich erste Hinweise auf einen Übergang zwischen den beiden Temperaturen für niederfrequente Schwingungen. Dieser Trend soll an weiteren Molekülen nachfolgend untersucht werden. Die Messanordnung soll durch Verwendung eines schmalbandigen Kerbfilters oder durch (flächenmäßig) größere Kantenfilter verbessert werden. Die Untersuchung kleiner Wasseraggregate lieferte Einblicke in die Energieübertragung zwischen gebundenen, miteinander gekoppelten $\mathrm{OH}$-Oszillatoren. Eine Fermi-Resonanz, die besonders stark für das Pentamer auftritt, konnte identifiziert werden und die Entwicklung der Kopplungskonstanten mit der Ringgröße erklären. Die Ergebnisse liefern Anreize zur Weiterentwicklung von volldimensionalen Modellpotentialen für Wasser.

Die Bestimmung thermodynamischer Größen aus Raman-Jetspektren wurde auf Systeme mit Konformationsisomeren angewendet. Es zeigte sich, dass die verwendete Methode besonders für Systeme mit großen Konversionsbarrieren geeignet ist. Am Beispiel von $N$-Methylformamid konnten freie Standardenthalpien und Standardentropien erhalten werden. Eine Untersuchung weiterer Systeme mit Konformationsisomeren könnte Aufschluss über die Anwendbarkeit dieser Auswertungsmethode in Abhängigkeit von der Umwandlungsbarriere liefern. 
Erste Studien der IR-Laser-Raman-Jetspektroskopie unter Nutzung verschiedener experimenteller Aufbauten am Beispiel des Methanoldimers zeigten bislang lediglich unspezifische Effekte. Durch Verbesserung des Aufbaus durch stärker fokussierende IROptiken soll die Überlappung zwischen IR- und Raman-Laser verbessert werden. Zudem kann das Signal-zu-Rausch-Verhältnis mittels eines leistungsstärkeren Raman-Lasers verbessert werden. Eine weitere Verbesserung des S/N-Verhältnisses können Studien weiterer geeigneter Testmoleküle hinsichtlich der maximal generierbaren Dimerkonzentration bei ähnlichem Absorptionsquerschnitten liefern. Mittels dieser Verbesserungen sollten die bestehenden Probleme des Aufbaus deutlich abgemildert werden.

Insgesamt wurden durch die vorliegende Arbeit wichtige Grundlagen zur weiteren Entwicklung der IR-Laser-Raman-Jetspektroskopie erarbeitet. 



\section{A Anhang}

\section{A.1 Publikationen}

Im Rahmen der vorliegenden Arbeit entstanden folgende Publikationen:

- K. E. Otto, Z. Xue, P. Zielke, M. A. Suhm, The Raman spectrum of isolated water clusters, Phys. Chem. Chem. Phys. 2014, 16, 9849-9858.

- M. Heger, K. E. Otto, R. A. Mata, M. A. Suhm, Bracketing subtle conformational energy differences between self-solvated and stretched trifluoropropanol, Phys. Chem. Chem. Phys., 2015, 17, 9899-9909.

\section{A.2 Chemikalienliste}

\begin{tabular}{llll}
\hline Substanz & Hersteller & CAS & Reinheit \\
\hline Wasser & Acros Organics & $7732-18-5$ & für HPLC \\
$\mathrm{D}_{2} \mathrm{O}$ & Aldrich & $7789-20-0$ & $99.9 \% \mathrm{D}$ \\
Methanol & Sigma-Aldrich & $67-56-1$ & $99.9 \%$ \\
$2,2,2-$ Trifluoroethanol & ABCR & $75-89-8$ & $99 \%$ \\
$3,3,3$-Trifluoropropanol & ABCR/Manchester Organics Ltd. & $2240-88-2$ & $97 \%$ \\
$N$-Methylformamid & ABCR & $123-39-7$ & $99 \%$ \\
Stickstoff & Air liquide & $7727-37-9$ & $99.999 \%$ \\
Helium & Linde/Air liquide & $7440-59-7$ & $99.996 \%$ \\
Argon & Air liquide & $7440-37-1$ & $99.999 \%$ \\
\hline
\end{tabular}




\section{Substanzspektren}

Wenn nicht anders angegeben, handelt es sich bei den Substanzspektren um Raman-Jetspektren, die am CurryJetspektrometer mit dem höher aufösenden Monochromator (MC2051) bei einer Eintrittsspaltbreite von $75 \mu \mathrm{m}$ im Stokes-Bereich mit senkrecht zur Streuebene polarisiertem Anregungslaser unter Nutzung des Stokes-Kantenfilters und einer $4 \times 0.15 \mathrm{~mm}^{2}$ Schlitzdüse aufgenommen wurden. Die Tabelle beinhaltet Angaben über den Dateinamen, der sich aus dem Datum der Form JJMMTT und einem Laufbuchstaben zusammensetzt, der Sättigertemperatur $\left(\vartheta_{\mathrm{S}}\right)$, dem Düsenabstand $d$, der Düsentemperatur $\left(\vartheta_{\mathrm{D}}\right)$ dem Trägergas, dem Stagnationsdruck $\left(p_{\mathrm{S}}\right)$, der Messzeit, der am Monochromator angezeigten Wellenlänge $\left(\lambda_{\mathrm{MC}}\right)$, dem Skalierungsfaktor für die Intensität (S.F.) sowie einer Spalte für sonstige Angaben.

Diese Spalte beinhaltet Angaben über abweichende Vorgehensweisen:

Aufnahme von Gasphasen im thermischen Gleichgewicht (GP) (wobei hier dann $p_{\mathrm{S}}$ der Druck in der Kammer ist), Verwendung eines anderen Rayleigh-Filters: Anti-Stokes-Kantenfilter (AS-Filter) oder (Polfilter), Nutzung des ReferenzRaman-Spektrometers (Praktikum), Nutzung des niedriger auflösenden Monochromators (MC205f) bei einer Eintrittsspaltbreite von $100 \mu \mathrm{m}$, Nutzung einer anderen Düse $\left(8 \times 0.05 \mathrm{~mm}^{2}\right)$ oder (Paraboldüse), parallel zur Streuebene polarisiertem Anregungslaser $(\lambda / 2)$, Nutzung des OPO-Lasers unter Angabe der OPO-Wellenzahl $\left(\widetilde{\nu}_{\mathrm{OPO}}\right)$ und bei Nutzung der Zylinderlinse zur Fokussierung des IR-Lasers die Angabe des Abstandes zwischen IR- und Raman-Fokus $\left(d_{\mathrm{f}}\right)$

\begin{tabular}{|c|c|c|c|c|c|c|c|c|c|c|c|}
\hline Abbildung & Spur & Datei(en) & $\vartheta_{\mathrm{S}} /{ }^{\circ} \mathrm{C}$ & $d / \mathrm{mm}$ & $\vartheta_{\mathrm{D}} /{ }^{\circ} \mathrm{C}$ & Trägergas & $p_{\mathrm{S}} /$ bar & Messzeit & $\lambda_{\mathrm{MC}} / \mathrm{nm}$ & S.F. & Sonstiges \\
\hline 4.1 (b) & & 130626_b & - & - & - & $2 \% \mathrm{~N}_{2}$ in $\mathrm{He}$ & 0.06 & $12 \times 1 \mathrm{~s}$ & 532.2 & 1.00 & GP, Polfilter \\
\hline $4.1(\mathrm{c})$ & schwarz & 130627_e & - & - & - & $2 \% \mathrm{~N}_{2}$ in $\mathrm{He}$ & 0.06 & $12 \times 5 \mathrm{~s}$ & 532.2 & 1.00 & GP \\
\hline 4.1 (c) & grau & $130627 \_\mathrm{d}$ & - & - & - & $2 \% \mathrm{~N}_{2}$ in $\mathrm{He}$ & 0.06 & $12 \times 5 \mathrm{~s}$ & 532.2 & 1.00 & GP, AS-Filter \\
\hline $4.3(\mathrm{~b})$ & & 130617_c & - & 1 & 25 & $2 \% \mathrm{~N}_{2}$ in $\mathrm{He}$ & 0.7 & $12 \times 2 \mathrm{~s}$ & 532.2 & 1.00 & Polfilter \\
\hline $4.3(\mathrm{c})$ & schwarz & 130423_a & - & 1 & 25 & $2 \% \mathrm{~N}_{2}$ in $\mathrm{He}$ & 0.7 & $6 \times 50 \mathrm{~s}$ & 539.2 & 1.00 & \\
\hline $4.3(\mathrm{c})$ & grau & 130423_d & - & 1 & 25 & $2 \% \mathrm{~N}_{2}$ in $\mathrm{He}$ & 0.7 & $12 \times 200 \mathrm{~s}$ & 525.1 & 1.00 & AS-Filter \\
\hline 4.5 (a) & schwarz & 140704_s_2 & - & - & - & Luft & 1 & $12 \times 100 \mathrm{~s}$ & 532.9 & 1.00 & GP, Praktikum \\
\hline 4.5 (a) & grau & 140704_a_2 & - & & & Luft & 1 & $12 \times 100 \mathrm{~s}$ & 528.0 & 1.00 & GP, Praktikum \\
\hline $4.5(\mathrm{~b})$ & & 140918_s & - & - & - & Luft & 1 & $18 \times 100 \mathrm{~s}$ & 530.7 & 1.00 & GP, Praktikum \\
\hline $4.5(\mathrm{c})$ & & 130612_a & - & - & - & Luft & 0.11 & $50 \times 1 \mathrm{~s}$ & 523.5 & 1.00 & GP, Polfilter \\
\hline 4.6 (a) & & 121015_b & 18 & 2 & 25 & $100 \% \mathrm{He}$ & 0.7 & $18 \times 200 \mathrm{~s}$ & 525.1 & 5.00 & AS-Filter \\
\hline $4.6(\mathrm{~b})$ & & 121015_b & 18 & 2 & 25 & $100 \% \mathrm{He}$ & 0.7 & $18 \times 100 \mathrm{~s}$ & 539.2 & 1.00 & \\
\hline 4.8 (a) & schwarz & 120206_b+07_b & -25 & 1 & 25 & $100 \% \mathrm{He}$ & 0.7 & $12 \times 100 \mathrm{~s}$ & 542.1 & 1.00 & \\
\hline $4.8(\mathrm{a})$ & grau & 120208_b & -25 & 1 & 80 & $100 \% \mathrm{He}$ & 0.7 & $6 \times 100 \mathrm{~s}$ & 542.1 & 1.00 & \\
\hline 4.8 (a) & hellgrau & $120206 \_\mathrm{d}$ & -25 & 1 & 150 & $100 \% \mathrm{He}$ & 0.7 & $6 \times 100 \mathrm{~s}$ & 542.1 & 1.00 & \\
\hline
\end{tabular}




\begin{tabular}{|c|c|c|c|c|c|c|c|c|c|c|c|}
\hline \multicolumn{12}{|c|}{ Fortsetzung Spektrenverzeichnis } \\
\hline Abbildung & Spur & Datei(en) & $\vartheta_{\mathrm{S}} /{ }^{\circ} \mathrm{C}$ & $d / \mathrm{mm}$ & $\vartheta_{\mathrm{D}} /{ }^{\circ} \mathrm{C}$ & Trägergas & $p_{\mathrm{S}} / \mathrm{bar}$ & Messzeit & $\lambda_{\mathrm{MC}} / \mathrm{nm}$ & S.F. & Sonstiges \\
\hline 4.8 (b) & schwarz & 120207_i & -25 & 1 & 25 & $100 \% \mathrm{He}$ & 0.7 & $12 \times 300 \mathrm{~s}$ & 519.8 & 25.00 & AS-Filter \\
\hline $4.8(\mathrm{~b})$ & grau & 120208_h & -25 & 1 & 80 & $100 \% \mathrm{He}$ & 0.7 & $12 \times 200 \mathrm{~s}$ & 519.8 & 25.00 & AS-Filter \\
\hline $4.8(\mathrm{~b})$ & hellgrau & $120206 \_\mathrm{j}+\mathrm{l}$ & -25 & 1 & 150 & $100 \% \mathrm{He}$ & 0.7 & $12 \times 200 \mathrm{~s}$ & 519.8 & 25.00 & AS-Filter \\
\hline 4.9 (a) & & \multicolumn{10}{|c|}{$=$ Abb. $4.8(\mathrm{a})$, hellgrau } \\
\hline 4.9 (b) & & $=\mathrm{Abb} .4 .8(\mathrm{~b}), \mathrm{h}$ & lgrau & & & & & & & & \\
\hline $4.9(\mathrm{c})$ & & \multicolumn{10}{|c|}{ (aus Spektrum 4.9 (a) berechnet) } \\
\hline \multirow[t]{5}{*}{5.1 , unten } & schwarz & 120813_d-a & 10 & 2 & 120 & $100 \% \mathrm{He}$ & 0.7 & $12 \times 300 \mathrm{~s}$ & 320.0 & 2.13 & MC205f \\
\hline & blau & $120813 \_c-a$ & 18 & 2 & 120 & $100 \% \mathrm{He}$ & 0.7 & $12 \times 300 \mathrm{~s}$ & 320.0 & 1.27 & MC205f \\
\hline & violett & 120813_b-a & 22 & 2 & 120 & $100 \% \mathrm{He}$ & 1.0 & $12 \times 300 \mathrm{~s}$ & 320.0 & 1.12 & MC205f \\
\hline & pink & 120808_f-a & 22 & 2 & 80 & $100 \% \mathrm{He}$ & 1.0 & $12 \times 300 \mathrm{~s}$ & 320.0 & 1.00 & MC205f \\
\hline & hellgrau & 120808_e-a & 22 & 2 & 25 & $100 \% \mathrm{He}$ & 1.0 & $12 \times 300 \mathrm{~s}$ & 320.0 & 1.00 & MC205f \\
\hline \multirow[t]{6}{*}{5.1 , oben, links } & schwarz & 120926_g & 10 & 2 & 120 & $100 \% \mathrm{He}$ & 0.7 & $18 \times 200 \mathrm{~s}$ & 660.1 & 2.00 & \\
\hline & blau & $120926 \_\mathrm{f}$ & 18 & 2 & 120 & $100 \% \mathrm{He}$ & 0.7 & $18 \times 100 \mathrm{~s}$ & 660.1 & 1.34 & \\
\hline & violett & 120926_e & 18 & 2 & 120 & $100 \% \mathrm{He}$ & 1.0 & $18 \times 100 \mathrm{~s}$ & 660.1 & 1.01 & \\
\hline & pink & 120926_d & 18 & 2 & 80 & $100 \% \mathrm{He}$ & 1.0 & $18 \times 100 \mathrm{~s}$ & 660.1 & 1.00 & \\
\hline & hellgrau & 120927_a & 18 & 2 & 25 & $100 \% \mathrm{He}$ & 1.0 & $18 \times 100 \mathrm{~s}$ & 660.1 & 1.05 & \\
\hline & hellblau & 120928_a & 18 & 2 & 25 & $20 \% \mathrm{Ar}$ in $\mathrm{He}$ & 0.7 & $12 \times 300 \mathrm{~s}$ & 660.1 & 1.20 & \\
\hline \multirow[t]{3}{*}{5.1 , oben, rechts } & hellgrau & aus Ref. 10 & 22 & 2 & 25 & $100 \% \mathrm{He}$ & 1.2 & $12 \times 300 \mathrm{~s}$ & 649 & 1.90 & Düse: $8 \times 0.05 \mathrm{~mm}^{2}$ \\
\hline & grau & aus Ref. 10 & 22 & 2 & 25 & $20 \% \mathrm{Ne}$ in $\mathrm{He}$ & 1.2 & $12 \times 300 \mathrm{~s}$ & 649 & 2.00 & Düse: $8 \times 0.05 \mathrm{~mm}^{2}$ \\
\hline & hellblau & aus Ref. 10 & 22 & 2 & 25 & $20 \% \mathrm{Ar}$ in $\mathrm{He}$ & 1.2 & $12 \times 300 \mathrm{~s}$ & 649 & 2.80 & Düse: $8 \times 0.05 \mathrm{~mm}^{2}$ \\
\hline \multirow[t]{2}{*}{$5.1, \mathrm{IR}$} & oben & aus Ref. 21 & \multicolumn{3}{|c|}{ Konzentration: $0.2 \%$} & $100 \% \mathrm{He}$ & 1.6 & 400 Scans & - & - & \\
\hline & unten & aus Ref. 21 & \multicolumn{3}{|c|}{ Konzentration: $0.7 \%$} & $100 \% \mathrm{He}$ & 1.0 & 361 Scans & - & - & \\
\hline \multirow[t]{5}{*}{5.2 , unten } & schwarz & 120903_e-f & 10 & 2 & 120 & $100 \% \mathrm{He}$ & 0.7 & $12 \times 300 \mathrm{~s}$ & 316.5 & 1.72 & MC205f \\
\hline & blau & 120903_d-f & 18 & 2 & 120 & $100 \% \mathrm{He}$ & 0.7 & $12 \times 300 \mathrm{~s}$ & 316.5 & 1.62 & MC205f \\
\hline & violett & 120903_c-a & 18 & 2 & 120 & $100 \% \mathrm{He}$ & 0.7 & $12 \times 300 \mathrm{~s}$ & 316.5 & 1.12 & MC205f \\
\hline & pink & 120903_b-a & 18 & 2 & 80 & $100 \% \mathrm{He}$ & 0.7 & $12 \times 300 \mathrm{~s}$ & 316.5 & 1.00 & MC205f \\
\hline & hellblau & 120904_b-c & 18 & 2 & 25 & $20 \% \mathrm{Ar}$ in $\mathrm{He}$ & 0.7 & $12 \times 300 \mathrm{~s}$ & 316.5 & 1.21 & MC205f \\
\hline \multirow[t]{7}{*}{5.2 , oben } & schwarz & 121004_a+b & 10 & 2 & 120 & $100 \% \mathrm{He}$ & 0.7 & $36 \times 100 \mathrm{~s}$ & 618.2 & 1.59 & \\
\hline & blau & 121002_e & 18 & 2 & 120 & $100 \% \mathrm{He}$ & 0.7 & $18 \times 100 \mathrm{~s}$ & 618.2 & 1.35 & \\
\hline & violett & 121002_d & 18 & 2 & 120 & $100 \% \mathrm{He}$ & 1.0 & $18 \times 100 \mathrm{~s}$ & 618.2 & 1.30 & \\
\hline & pink & 121001_e & 18 & 2 & 80 & $100 \% \mathrm{He}$ & 1.0 & $18 \times 100 \mathrm{~s}$ & 618.2 & 1.00 & \\
\hline & hellgrau & aus Ref. 10 & 22 & 2 & 25 & $100 \% \mathrm{He}$ & 1.1 & $12 \times 300 \mathrm{~s}$ & 616 & 2.65 & Düse: $8 \times 0.05 \mathrm{~mm}^{2}$ \\
\hline & grau & aus Ref. 10 & 22 & 2 & 25 & $20 \% \mathrm{Ne}$ in $\mathrm{He}$ & 1.1 & $12 \times 300 \mathrm{~s}$ & 616 & 3.00 & Düse: $8 \times 0.05 \mathrm{~mm}^{2}$ \\
\hline & hellblau & aus Ref. 10 & 22 & 2 & 25 & $20 \% \mathrm{Ar}$ in $\mathrm{He}$ & 1.1 & $12 \times 300 \mathrm{~s}$ & 616 & 4.20 & Düse: $8 \times 0.05 \mathrm{~mm}^{2}$ \\
\hline \multirow[t]{2}{*}{$5.2, \mathrm{IR}$} & oben & aus Ref. 21 & \multicolumn{3}{|c|}{ Konzentration: $0.16 \%$} & $100 \% \mathrm{Ar}$ & 0.5 & 99 Scans & - & & \\
\hline & unten & aus Ref. 21 & \multicolumn{3}{|c|}{ Konzentration: $0.08 \%$} & $100 \% \mathrm{He}$ & 2.0 & $<150$ Scans & - & & \\
\hline \multirow[t]{2}{*}{5.3 , links } & oben & 120808_d-a & 22 & 2 & 25 & $100 \% \mathrm{He}$ & 1.0 & $12 \times 300 \mathrm{~s}$ & 320.0 & 1.00 & \\
\hline & unten & \multicolumn{10}{|c|}{$=$ Abb. 5.3 , links oben -2.2 Abb. 5.1 , unten (violett) } \\
\hline \multirow[t]{2}{*}{5.3 , rechts } & oben & \multirow{2}{*}{\multicolumn{10}{|c|}{$\begin{array}{l}=\text { Abb. } 5.2, \text { unten }(\text { grau }) \\
=\text { Abb. } 5.2, \text { unten }(\text { grau })-2.8 \times \text { Abb. } 5.2 \text {, unten (hellgrau) }\end{array}$}} \\
\hline & unten & & & & & & & & & & \\
\hline \multicolumn{2}{|l|}{5.4} & 120927_b & 18 & 2 & 25 & $100 \% \mathrm{He}$ & 0.7 & $18 \times 100 \mathrm{~s}$ & 585.0 & 1.00 & \\
\hline \multirow[t]{2}{*}{5.5} & oben & $120920 \_\mathrm{c}+\mathrm{d}+\mathrm{e}$ & 18 & 0.5 & 25 & $100 \% \mathrm{He}$ & 0.7 & $30 \times 300 \mathrm{~s}$ & 423.8 & 1.00 & MC205f \\
\hline & unten & 120920_g & 18 & 0.5 & 25 & $100 \% \mathrm{He}$ & 0.7 & $13 \times 60 \mathrm{~s}$ & 316.5 & & MC205f \\
\hline 6.1 (a) & & $111103 \_c$ & 15 & 1 & 150 & $100 \% \mathrm{He}$ & 0.7 & $15 \times 100 \mathrm{~s}$ & 653.1 & 1.00 & \\
\hline
\end{tabular}




\begin{tabular}{|c|c|c|c|c|c|c|c|c|c|c|c|}
\hline \multicolumn{12}{|c|}{ Fortsetzung Spektrenverzeichnis } \\
\hline Abbildung & Spur & Datei(en) & $\vartheta_{\mathrm{S}} /{ }^{\circ} \mathrm{C}$ & $d / \mathrm{mm}$ & $\vartheta_{\mathrm{D}} /{ }^{\circ} \mathrm{C}$ & Trägergas & $p_{\mathrm{S}} / \mathrm{bar}$ & Messzeit & $\lambda_{\mathrm{MC}} / \mathrm{nm}$ & S.F. & Sonstiges \\
\hline $6.1(\mathrm{~b})$ & & 111103_f & 15 & 1 & 150 & $100 \% \mathrm{He}$ & 0.7 & $6 \times 30 \mathrm{~s}$ & 637.2 & 1.00 & \\
\hline $6.2(\mathrm{a})$ & schwarz & 120206_h & -25 & 1 & 150 & $100 \% \mathrm{He}$ & 0.7 & $6 \times 100 \mathrm{~s}$ & 564.0 & 1.00 & \\
\hline & grau & 120206_i & -25 & 1 & 150 & $100 \% \mathrm{He}$ & 0.7 & $6 \times 100 \mathrm{~s}$ & 564.0 & 1.00 & $\lambda / 2$ \\
\hline $6.2(\mathrm{~b})$ & schwarz & 111103_o & -10 & 1 & 150 & $100 \% \mathrm{He}$ & 0.7 & $6 \times 100 \mathrm{~s}$ & 572.0 & 0.50 & \\
\hline & hellgrau & 111103_l & 15 & 1 & 150 & $100 \% \mathrm{He}$ & 0.7 & $6 \times 50 \mathrm{~s}$ & 572.0 & 0.18 & \\
\hline 6.3 & schwarz & 120206_d & -25 & 1 & 150 & $100 \% \mathrm{He}$ & 0.7 & $6 \times 100 \mathrm{~s}$ & 542.1 & 1.00 & \\
\hline & grau & 120206_e & -25 & 1 & 150 & $100 \% \mathrm{He}$ & 0.7 & $6 \times 100 \mathrm{~s}$ & 542.1 & 1.00 & $\lambda / 2$ \\
\hline 6.5 , links & schwarz & 130116_b & -25 & 1 & 25 & $100 \% \mathrm{He}$ & 0.7 & $18 \times 100 \mathrm{~s}$ & 633.1 & 0.14 & \\
\hline & grau & 130116_c & -25 & 1 & 80 & $100 \% \mathrm{He}$ & 0.7 & $18 \times 100 \mathrm{~s}$ & 633.1 & 0.17 & \\
\hline & hellgrau & 130116_d & -25 & 1 & 150 & $100 \% \mathrm{He}$ & 0.7 & $18 \times 100 \mathrm{~s}$ & 633.1 & 0.29 & \\
\hline 6.5 , rechts & schwarz & 120201_e & -25 & 1 & 25 & $100 \% \mathrm{He}$ & 0.7 & $6 \times 100 \mathrm{~s}$ & 558.6 & 0.05 & \\
\hline & grau & 120201_d & -25 & 1 & 80 & $100 \% \mathrm{He}$ & 0.7 & $6 \times 100 \mathrm{~s}$ & 558.6 & 0.06 & \\
\hline & hellgrau & 120201_f & -25 & 1 & 150 & $100 \% \mathrm{He}$ & 0.7 & $6 \times 100 \mathrm{~s}$ & 558.6 & 0.06 & \\
\hline 6.7 & schwarz & 130717_a & 12 & 2 & 25 & $100 \% \mathrm{He}$ & 0.7 & $36 \times 100 \mathrm{~s}$ & 644.0 & 0.62 & \\
\hline & grau & 130717_b & 12 & 2 & 80 & $100 \% \mathrm{He}$ & 0.7 & $36 \times 100 \mathrm{~s}$ & 644.0 & 0.75 & \\
\hline & hellgrau & 130717_e & 12 & 2 & 150 & $100 \% \mathrm{He}$ & 0.7 & $36 \times 100 \mathrm{~s}$ & 644.0 & 0.70 & \\
\hline 6.9 , oben & schwarz & 111012_b & 60 & 1 & 80 & $100 \% \mathrm{He}$ & 0.7 & $6 \times 100 \mathrm{~s}$ & 644.0 & 0.03 & \\
\hline & grau & 111012_h & 60 & 1 & 120 & $100 \% \mathrm{He}$ & 0.7 & $12 \times 100 \mathrm{~s}$ & 644.0 & 0.02 & \\
\hline & hellgrau & $111012 \_c$ & 60 & 1 & 150 & $100 \% \mathrm{He}$ & 0.7 & $6 \times 100 \mathrm{~s}$ & 644.0 & 0.04 & \\
\hline 6.9 , unten & schwarz & $=6.9$, oben $($ schwarz & & & & & & & & & \\
\hline & hellgrau & $130716 \_c$ & 15 & 1 & 25 & $100 \% \mathrm{He}$ & 0.7 & $18 \times 100 \mathrm{~s}$ & 644.0 & 0.19 & \\
\hline 7.1 (a) & schwarz & 140116_f & -25 & 2.5 & 25 & $100 \% \mathrm{He}$ & 0.7 & $6 \times 100 \mathrm{~s}$ & 657.9 & 1.00 & Paraboldüse \\
\hline & rot & 140116_g & -25 & 2.5 & 25 & $100 \% \mathrm{He}$ & 0.7 & $6 \times 100 \mathrm{~s}$ & 657.9 & 1.00 & $\begin{array}{l}\text { Paraboldüse } \\
\widetilde{\nu}_{\mathrm{OPO}}: 3575 \mathrm{~cm}^{-1}\end{array}$ \\
\hline 7.1 (b) & schwarz & 140116_a & -25 & 3 & 25 & $100 \% \mathrm{He}$ & 0.7 & $6 \times 100 s$ & 657.9 & 1.00 & Paraboldüse \\
\hline & rot & 140116_b & -25 & 3 & 25 & $100 \% \mathrm{He}$ & 0.7 & $6 \times 100 \mathrm{~s}$ & 657.9 & 1.00 & $\begin{array}{l}\text { Paraboldüse } \\
\widetilde{\nu}_{\mathrm{OPO}}: 3575 \mathrm{~cm}^{-1}\end{array}$ \\
\hline 7.1 (c) & schwarz & 140116_e & -25 & 3.5 & 25 & $100 \% \mathrm{He}$ & 0.7 & $6 \times 100 \mathrm{~s}$ & 657.9 & 1.00 & Paraboldüse \\
\hline & rot & 140116_c & -25 & 3.5 & 25 & $100 \% \mathrm{He}$ & 0.7 & $6 \times 100 \mathrm{~s}$ & 657.9 & 1.00 & $\begin{array}{l}\text { Paraboldüse } \\
\widetilde{\nu}_{\mathrm{OPO}}: 3575 \mathrm{~cm}^{-1}\end{array}$ \\
\hline 7.2 & & $=$ Abb. 7.1 & & & & & & & & & \\
\hline 7.3 (a) & schwarz & 140408_i & -25 & 1 & 25 & $100 \% \mathrm{He}$ & 0.7 & $6 \times 100 \mathrm{~s}$ & 657.9 & 1.00 & \\
\hline & rot & $140408 \_\mathrm{j}$ & -25 & 1 & 25 & $100 \% \mathrm{He}$ & 0.7 & $6 \times 100 \mathrm{~s}$ & 657.9 & 1.00 & $\begin{array}{l}d_{\mathrm{f}}:-1 \mathrm{~mm} \\
\widetilde{\nu}_{\mathrm{OPO}}: 3575 \mathrm{~cm}^{-1}\end{array}$ \\
\hline 7.3 (b) & schwarz & 140408_k & -25 & 1 & 25 & $100 \% \mathrm{He}$ & 0.7 & $6 \times 100 s$ & 657.9 & 1.00 & \\
\hline & rot & 140408_l & -25 & 1 & 25 & $100 \% \mathrm{He}$ & 0.7 & $6 \times 100 \mathrm{~s}$ & 657.9 & 1.00 & $\begin{array}{l}d_{\mathrm{f}}:-0.5 \mathrm{~mm} \\
\widetilde{\nu}_{\mathrm{OPO}}: 3575 \mathrm{~cm}^{-1}\end{array}$ \\
\hline 7.3 (c) & schwarz & 140408_i & -25 & 1 & 25 & $100 \% \mathrm{He}$ & 0.7 & $6 \times 100 \mathrm{~s}$ & 657.9 & 1.00 & \\
\hline & rot & 140408_f & -25 & 1 & 25 & $100 \% \mathrm{He}$ & 0.7 & $6 \times 100 \mathrm{~s}$ & 657.9 & 1.00 & $\begin{array}{l}d_{\mathrm{f}}: 0 \mathrm{~mm} \\
\widetilde{\nu}_{\mathrm{OPO}}: 3575 \mathrm{~cm}^{-1}\end{array}$ \\
\hline 7.3 (d) & schwarz & 140408_m & -25 & 1 & 25 & $100 \% \mathrm{He}$ & 0.7 & $6 \times 100 \mathrm{~s}$ & 657.9 & 1.00 & \\
\hline & rot & 140408_n & -25 & 1 & 25 & $100 \% \mathrm{He}$ & 0.7 & $6 \times 100 \mathrm{~s}$ & 657.9 & 1.00 & $\begin{array}{l}d_{\mathrm{f}}: 0.5 \mathrm{~mm} \\
\widetilde{\nu}_{\mathrm{OPO}}: 3575 \mathrm{~cm}^{-1}\end{array}$ \\
\hline 7.4 (a) & schwarz & 140410_a & -25 & 1 & 25 & $100 \% \mathrm{He}$ & 0.7 & $6 \times 100 \mathrm{~s}$ & 657.9 & 1.00 & \\
\hline
\end{tabular}

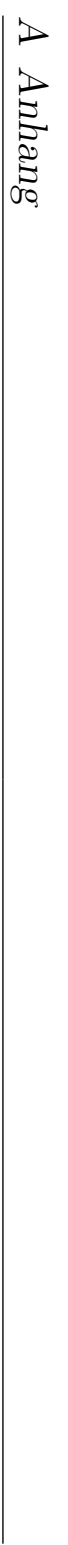




\begin{tabular}{|c|c|c|c|c|c|c|c|c|c|c|c|}
\hline \multicolumn{12}{|c|}{ Fortsetzung Spektrenverzeichnis } \\
\hline Abbildung & Spur & Datei(en) & $\vartheta_{\mathrm{S}} /{ }^{\circ} \mathrm{C}$ & $d / \mathrm{mm}$ & $\vartheta_{\mathrm{D}} /{ }^{\circ} \mathrm{C}$ & Trägergas & $p_{\mathrm{S}} / \mathrm{bar}$ & Messzeit & $\lambda_{\mathrm{MC}} / \mathrm{nm}$ & S.F. & Sonstiges \\
\hline & rot & 140410_b & -25 & 1 & 25 & $100 \% \mathrm{He}$ & 0.7 & $6 \times 100 \mathrm{~s}$ & 657.9 & 1.00 & $\begin{array}{l}d_{\mathrm{f}}: 0 \mathrm{~mm} \\
\widetilde{\nu}_{\mathrm{OPO}}: 3575 \mathrm{~cm}^{-1}\end{array}$ \\
\hline $7.4(\mathrm{~b})$ & schwarz & 140514_f & -25 & 1 & 25 & $100 \% \mathrm{Ar}$ & 0.5 & $12 \times 100 \mathrm{~s}$ & 657.9 & 2.30 & \\
\hline & rot & 140514_g & -25 & 1 & 25 & $100 \% \mathrm{Ar}$ & 0.5 & $12 \times 100 \mathrm{~s}$ & 657.9 & 2.30 & 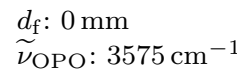 \\
\hline $7.4(\mathrm{c})$ & schwarz & 140514 j & -25 & 0.4 & 25 & $100 \% \mathrm{Ar}$ & 0.5 & $12 \times 100 \mathrm{~s}$ & 657.9 & 0.88 & \\
\hline & rot & 140514_k & -25 & 0.4 & 25 & $100 \% \mathrm{Ar}$ & 0.5 & $12 \times 100 \mathrm{~s}$ & 657.9 & 0.88 & $\begin{array}{l}d_{\mathrm{f}}: 0 \mathrm{~mm} \\
\widetilde{\nu}_{\mathrm{OPO}}: 3575 \mathrm{~cm}^{-1}\end{array}$ \\
\hline 7.5 (a) & & $=$ Abb. $7.4(\mathrm{c})$ & & & & & & & & & \\
\hline 7.5 (b) & schwarz & 140515_i & -25 & 0.4 & 25 & $100 \% \mathrm{Ar}$ & 0.5 & $12 \times 100 \mathrm{~s}$ & 657.9 & 1.00 & \\
\hline & rot & 140515_h & -25 & 0.4 & 25 & $100 \% \mathrm{Ar}$ & 0.5 & $12 \times 100 \mathrm{~s}$ & 657.9 & 1.00 & $\begin{array}{l}d_{\mathrm{f}}: 0 \mathrm{~mm} \\
\widetilde{\nu}_{\mathrm{OPO}}: 3500 \mathrm{~cm}^{-1}\end{array}$ \\
\hline 7.5 (c) & schwarz & 140516_c & -25 & 0.4 & 25 & $100 \% \mathrm{Ar}$ & 0.5 & $12 \times 100 \mathrm{~s}$ & 657.9 & 1.00 & \\
\hline & rot & 140516_d & -25 & 0.4 & 25 & $100 \% \mathrm{Ar}$ & 0.5 & $12 \times 100 \mathrm{~s}$ & 657.9 & 1.00 & $\begin{array}{l}d_{\mathrm{f}}: 0 \mathrm{~mm} \\
\widetilde{\nu}_{\mathrm{OPO}}: 3474 \mathrm{~cm}^{-1}\end{array}$ \\
\hline 7.6 (a) & schwarz & 140527_a & -25 & 0.4 & 25 & $100 \% \mathrm{Ar}$ & 0.5 & $12 \times 100 \mathrm{~s}$ & 657.9 & 1.00 & \\
\hline & grau & 140527_b & -25 & 0.4 & 80 & $100 \% \mathrm{Ar}$ & 0.5 & $12 \times 100 \mathrm{~s}$ & 657.9 & 1.00 & \\
\hline & hellgrau & 140527_f & -25 & 0.4 & 150 & $100 \% \mathrm{Ar}$ & 0.5 & $12 \times 100 \mathrm{~s}$ & 657.9 & 1.00 & \\
\hline $7.6(\mathrm{~b})$ & & $=$ Abb. $7.5(\mathrm{~b})$ & & & & & & & & & \\
\hline A.7.1 (a) & & 130116_b & -25 & 1 & 25 & $100 \% \mathrm{He}$ & 0.7 & $18 \times 100 \mathrm{~s}$ & 633.1 & 1.00 & \\
\hline A.7.1 (b) & & 120201_e & -25 & 1 & 25 & $100 \% \mathrm{He}$ & 0.7 & $6 \times 100 \mathrm{~s}$ & 558.6 & 1.00 & \\
\hline A. 7.2 & & 121101_f & -25 & 1 & 25 & $100 \% \mathrm{He}$ & 0.7 & $24 \times 100 \mathrm{~s}$ & 577.3 & 1.00 & \\
\hline A.7.3 & & $=$ Abb. 6.9, unten & & & & & & & & & \\
\hline A.7.4 & & $111012 \_\mathrm{j}$ & 60 & 1 & 120 & $100 \% \mathrm{He}$ & 0.7 & $6 \times 50 \mathrm{~s}$ & 583.3 & 1.00 & \\
\hline A.8.1 (a) & & $=$ Abb. $7.4(\mathrm{c})$ & & & & & & & & & \\
\hline A.8.1 (b) & schwarz & 140515_a & -25 & 0.4 & 25 & $100 \% \mathrm{Ar}$ & 0.5 & $12 \times 100 \mathrm{~s}$ & 657.9 & 1.00 & \\
\hline & & 140515_b & -25 & 0.4 & 25 & $100 \% \mathrm{Ar}$ & 0.5 & $12 \times 100 \mathrm{~s}$ & 657.9 & 1.00 & $\begin{array}{l}d_{\mathrm{f}}: 0 \mathrm{~mm} \\
\widetilde{\nu}_{\mathrm{OPO}}: 3575 \mathrm{~cm}^{-1}\end{array}$ \\
\hline A.8.1 (c) & schwarz & 140515_c & -25 & 0.4 & 25 & $100 \% \mathrm{Ar}$ & 0.5 & $12 \times 100 \mathrm{~s}$ & 657.9 & 1.00 & \\
\hline & & 140515_d & -25 & 0.4 & 25 & $100 \% \mathrm{Ar}$ & 0.5 & $12 \times 100 \mathrm{~s}$ & 657.9 & 1.00 & $\begin{array}{l}d_{\mathrm{f}}: 0 \mathrm{~mm} \\
\widetilde{\nu}_{\mathrm{OPO}}: 3575 \mathrm{~cm}^{-1}\end{array}$ \\
\hline
\end{tabular}




\section{OPO-Spektren}

Das Verzeichnis der FTIR-Spektren zur Bestimmung der Wellenzahl des OPO-Lasers beinhaltet den Dateinamen und die Scanzahl. Die weiteren Messparameter sind in Anhang A.4.2 aufgeführt. Die Dateinamen sind wie folgt aufgebaut:

JJJJMMTT_OPO_Laufzahl bzw. für die frühen Testspektren:

JJJJMMTT_test_Laufbuchstabe.

\begin{tabular}{|c|c|c|}
\hline \multicolumn{3}{|c|}{ OPO-FTIR-Spektren } \\
\hline Abbildung & Datei & Scanzahl \\
\hline $3.9(\mathrm{a})$ & 20130604_test_k & 30 \\
\hline $3.9(\mathrm{~b})$ & 20130604_test_l & 30 \\
\hline $3.9(\mathrm{c})$ & 20130604_test_m & 30 \\
\hline $3.9(\mathrm{~d})$ & 20130604_test_n & 30 \\
\hline 3.10 (grau) & 20130604_test_j & 30 \\
\hline $3.10(\mathrm{a})$ & 20130909_OPO_9 & 100 \\
\hline 3.10(b) & 20130909_OPO_10 & 100 \\
\hline $3.10(\mathrm{c})$ & 20130909_OPO_11 & 100 \\
\hline 7.1 & 20140116_OPO_1 & \\
\hline 7.3 & 20140408_OPO_1 & 100 \\
\hline 7.4 & 20140410_OPO_5 & 100 \\
\hline 7.5 (a) & 20140514_OPO_1 & 100 \\
\hline $7.5(\mathrm{~b})$ & 20140515_OPO_8 & 100 \\
\hline $7.5(\mathrm{c})$ & 20140516_OPO_6 & 100 \\
\hline A. $4.5(\mathrm{a})$ & 20130924_OPO_19 & 100 \\
\hline A. $4.5(\mathrm{~b})$ & 20130924_OPO_18 & 100 \\
\hline A.4.5 (c) & 20130924_OPO_17 & 100 \\
\hline A.4.5 (d) & 20130924_OPO_16 & 100 \\
\hline A.8.1 & 20140515_OPO_1 & 100 \\
\hline
\end{tabular}




\section{A.4 Experimentelles}

\section{A.4.1 Ergänzende Abbildungen}

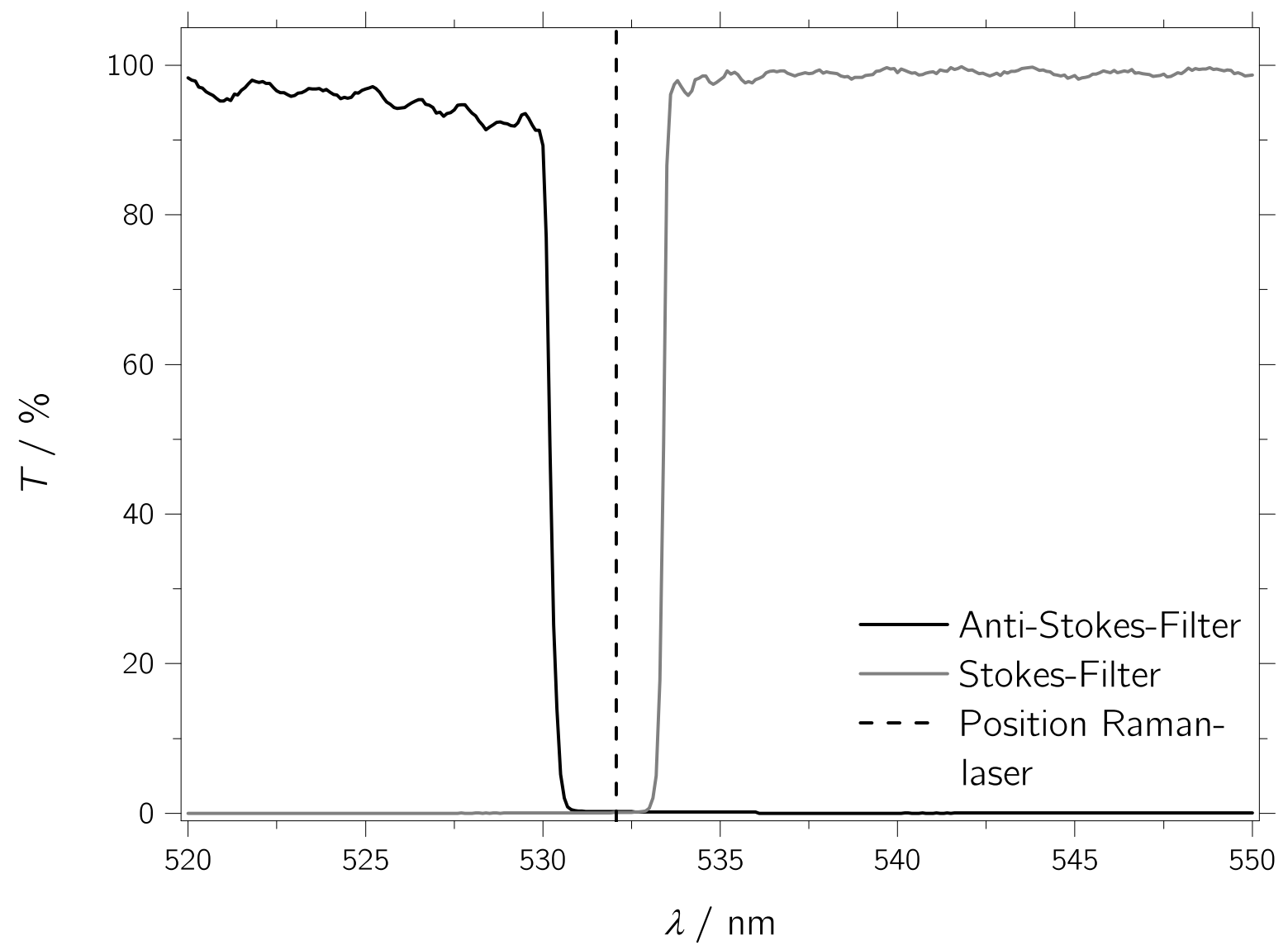

Abbildung A.4.1: Transmissionsspektren der verwendeten Rayleighfilter aufgenommen mittels UV/VIS-Spektrometer (Shimadzu UV-2450 (PC), Shimadzu Corporation, Aufnahmebereich: 550-520 nm, Auflösung: $0.1 \mathrm{~nm}$, Datenaufnahmeintervall: $0.1 \mathrm{~nm}$ ). Die Transmissionseingenschaften des Stokes-Filters (LOT-Quantum Design GmbH, REFUS532-25 USLR Ramankantenfilter, OD 6.0@532.0 nm, T>90\%, $\lambda>533.7 \mathrm{~nm}$ ) sind um einige Prozentpunkte besser als die des Anti-Stokes-Filters (LOT-Oriel Quantum Design GmbH, SPF532-25, USLR Raman-Kurzpassfilter, OD $>6 @ 532.0 \mathrm{~nm}, T>90 \%, \lambda<529.9 \mathrm{~nm})$. Für vergleichende Messungen der Intensität von Stokes- und Anti-Stokes-Übergängen muss dieser Befund miteinbezogen werden. 


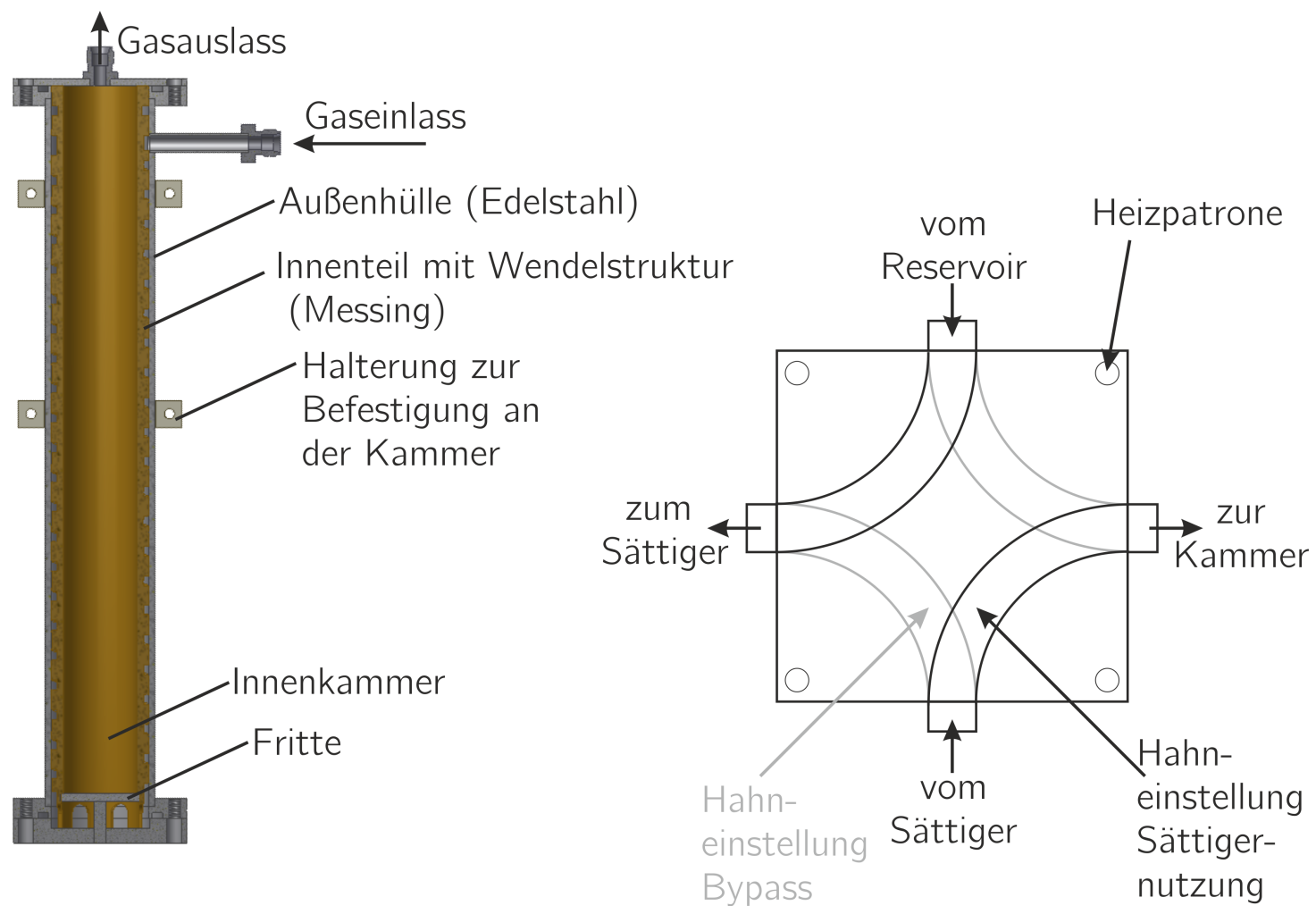

Abbildung A.4.2: Technische Zeichnung (Schnitt) des beheizbaren Sättigers. ${ }^{170}$ Das Trägergas strömt über den Gaseinlass in den Sättiger, durch die Wendelstuktur auf der Außenseite des Innenteils wird das Gas nach unten zur Fritte geleitet und hierbei erwärmt. Nachdem es die Fritte durchtreten hat, kommt es in Kontakt mit der Substanz. Das Substanz-Trägergas-Gemisch wird durch den Gasauslass zunächst zurück zum 4-Wege Hahn geleitet und anschließend in die Kammer expandiert. (Nicht dargestellt sind die Heizbänder, die um die Außenhülle des Metallsättigers gewickelt sind.) Auf der rechten Seite befindet sich eine schematische Darstellung des 4-Wege Kugelhahns. In grau eingezeichnet ist die Hahneinstellung für den Bypass des Sättigers und in schwarz die Einstellung bei Nutzung des Sättigers. 


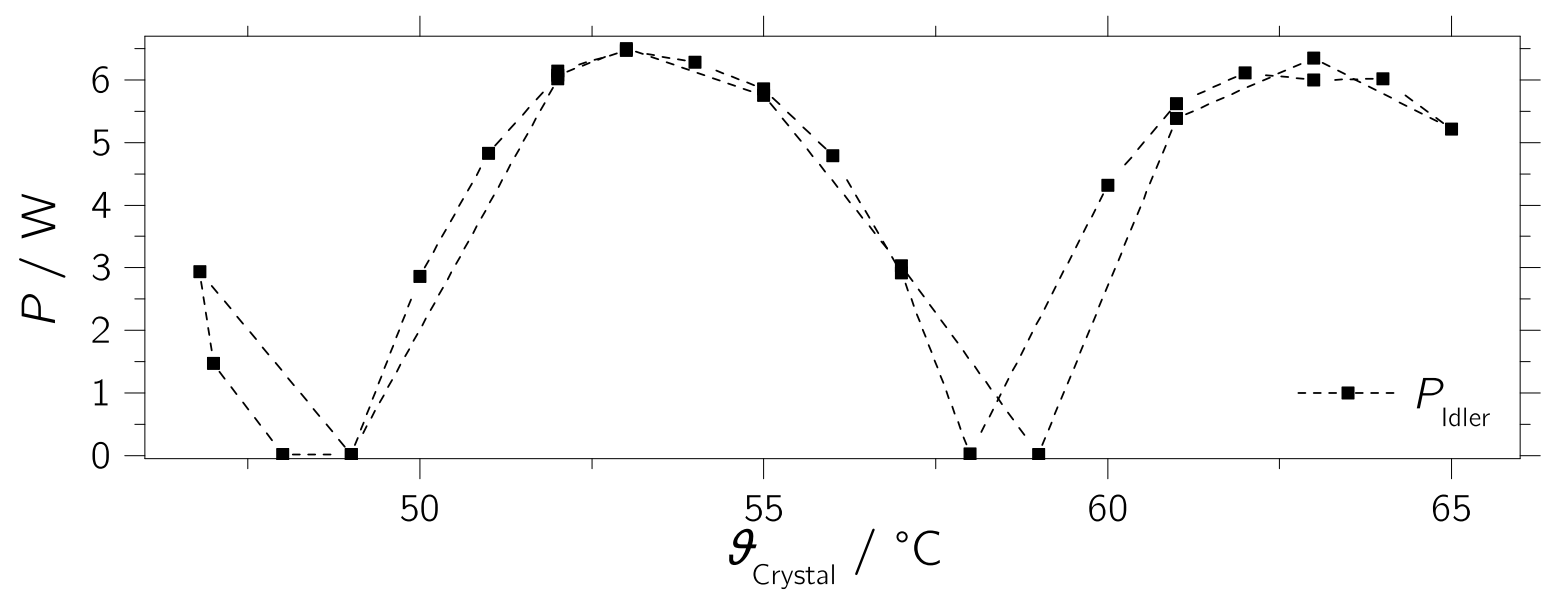

Abbildung A.4.3: Abhängigkeit der Leistung des OPO-Lasers von der Temperatur bei konstantem Etalonwinkel von $1.25^{\circ}$ und konstanter Kristallposition.

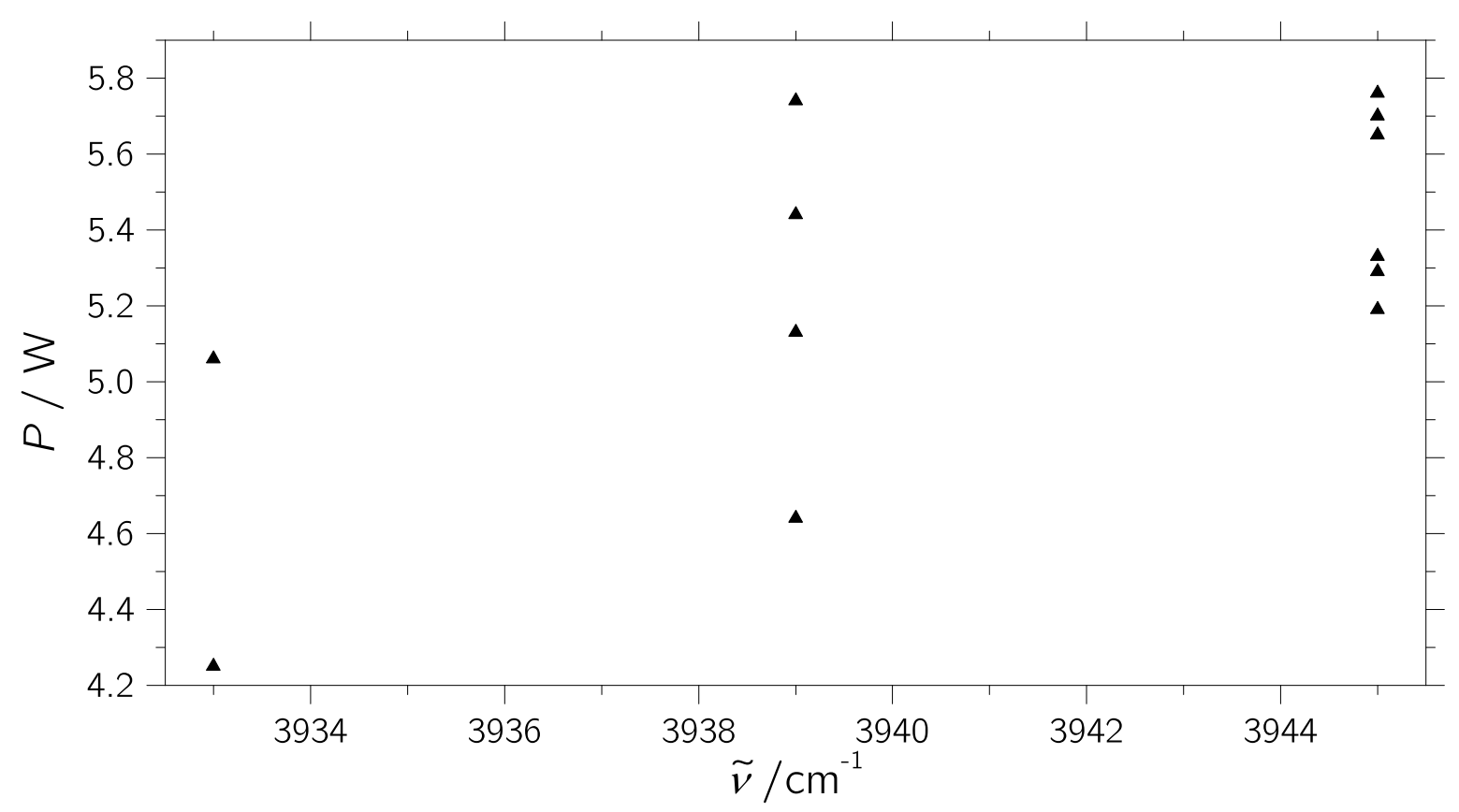

Abbildung A.4.4: Darstellung der Leistungsunterschiede, die bei gleichbleibender Wellenzahl auftreten, wenn die Kristallposition um einen Zählerstand von \pm 50 variiert wird. Die Leistung und die Wellenzahl wurden für eine Kristalltemperatur von $57^{\circ} \mathrm{C}$ bestimmt. Außerdem zeigt sich die Modenstruktur des Lasers mit einem Modenabstand von $6 \mathrm{~cm}^{-1}$ im gezeigten Wellenzahlbereich. 


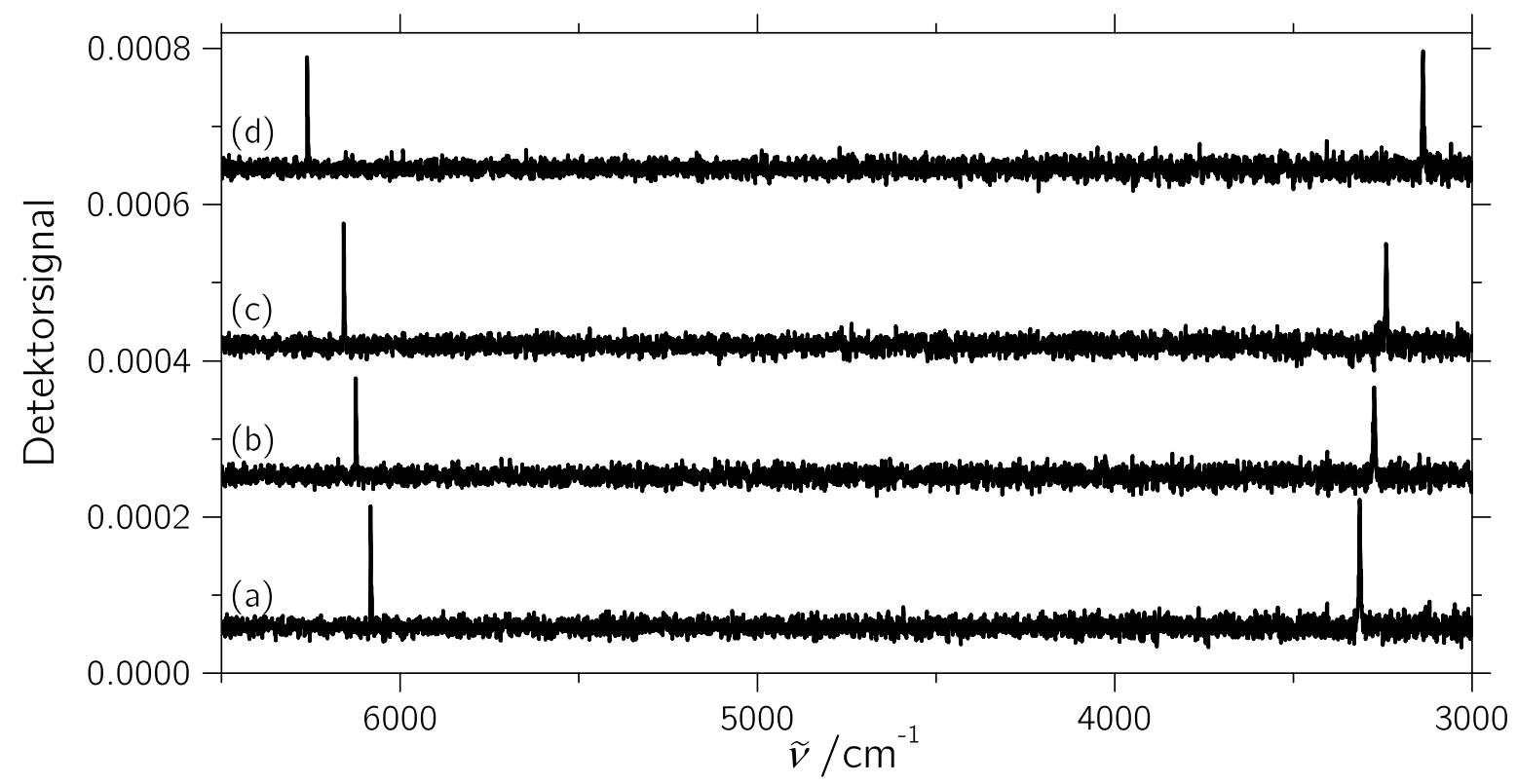

Abbildung A.4.5: FTIR-Spektren von vier verschiedenen OPO-Einstellungen. Die Signalstrahlung zeigt sich als zusätzlicher Peak um $6000 \mathrm{~cm}^{-1}$. In Tabelle A.4.1 sind die zugehörigen Wellenzahlen der jeweiligen Peaks aufgelistet.

\begin{tabular}{ccccc}
\hline Spektrum & $\widetilde{\nu}_{\mathrm{I}} / \mathrm{cm}^{-1}$ & $\widetilde{\nu}_{\mathrm{S}} / \mathrm{cm}^{-1}$ & $\widetilde{\nu}_{\mathrm{I}}+\widetilde{\nu}_{\mathrm{S}} / \mathrm{cm}^{-1}$ & $\frac{1}{\widetilde{\nu}_{\mathrm{I}}+\widetilde{\nu}_{\mathrm{S}}} / \mathrm{nm}$ \\
\hline (a) & 3138 & 6260 & 9398 & 1064 \\
(b) & 3240 & 6158 & 9398 & 1064 \\
(c) & 3274 & 6124 & 9398 & 1064 \\
(d) & 3315 & 6083 & 9398 & 1064 \\
\hline
\end{tabular}

Tabelle A.4.1: Wellenzahlen der Idler- $\left(\widetilde{\nu}_{\mathrm{I}}\right)$ und Signalstrahlung $\left(\widetilde{\nu}_{\mathrm{S}}\right)$ der in Abbildung A.4.5 gezeigten Spektren, sowie die Summe beider und der Kehrwert der Summe, der der Pumpwellenlänge entspricht. 


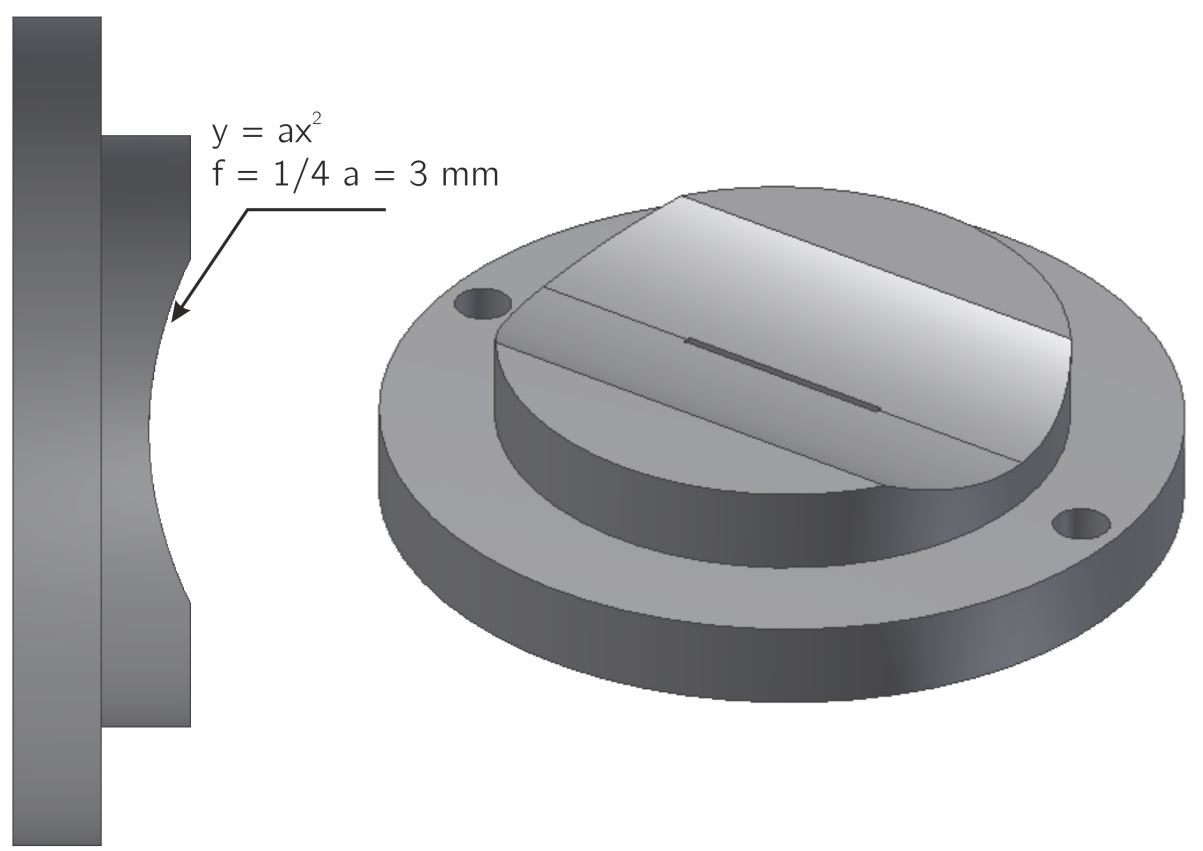

Abbildung A.4.6: Technische Zeichnung der Paraboldüse ${ }^{171}$ angefertigt im Zeichenbüro des Instituts für Physikalische Chemie der Universität Göttingen. Die Form entspricht einer Parabolrinne mit einer Brennweite von $3 \mathrm{~mm}$. Die Tiefe der Rinne beträgt $0.7 \mathrm{~mm}$. 
a)

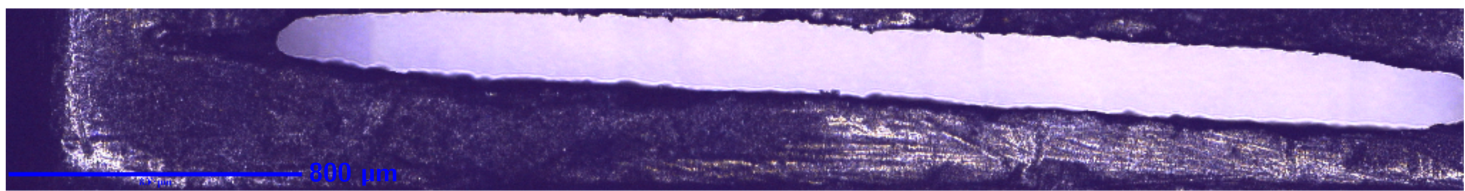

b)

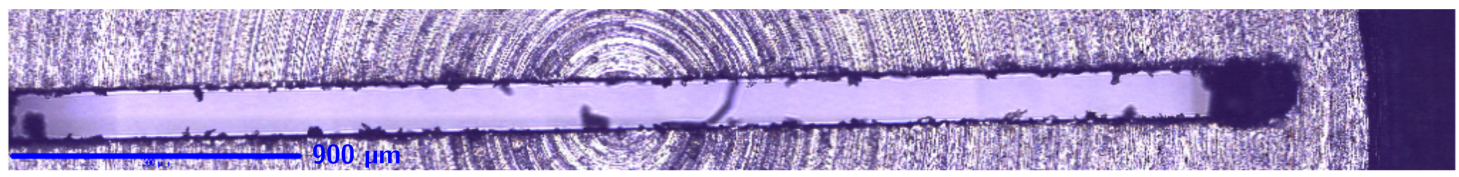

c)

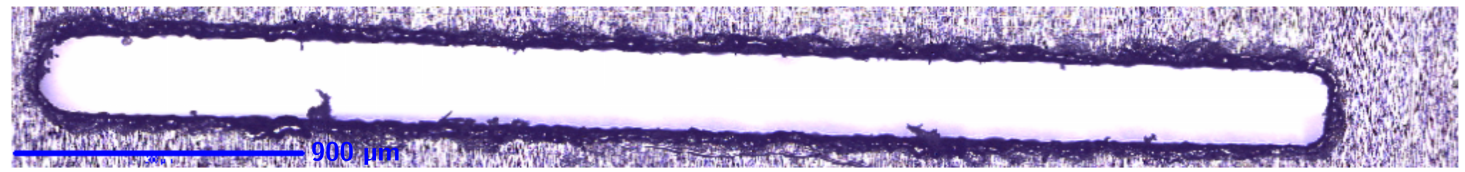

Abbildung A.4.7: Düsenprofile a) der alten Schlitzdüse, b) der neuen Schlitzdüse und c) der Paraboldüse aufgenommen mit 10 facher Vergrößerung mittels Raman-Mikroskop (alpha300M+, WITec GmbH). Die neue Schlitzdüse wurde durch Drahterosion hergestellt während die alte durch Senkerosion mittels eines Blechstreifens gefertigt wurde. ${ }^{48} \mathrm{Im}$ Vergleich zur alten Düse zeigt die neue eine gleichmäßigere Schlitzbreite. Die Öffnung der Paraboldüse ist etwas breiter als die anderen beiden, was sich durch einen höheren Gasdurchsatz während der Expansion bemerkbar macht.

\section{A.4.2 Experimentelle Parameter für die Aufnahme von FTIR-Spektren zur Bestimmung der Wellenzahl des OPO-Lasers}

\begin{tabular}{|c|c|c|c|}
\hline \multicolumn{4}{|c|}{ Instrument Parameter } \\
\hline Spektrometertyp & Vector 22 & & \\
\hline \multicolumn{4}{|l|}{ Optikparameter } \\
\hline Beamsplitter & $\mathrm{KBr}$ & Scanner velocity & $10 \mathrm{kHz}$ \\
\hline Detector setting & DTGS & Low Pass Filter & $16 \mathrm{kHz}$ \\
\hline Aperture setting & open & Measurement channel & Front \\
\hline \multicolumn{4}{|c|}{ Akquisitionsparameter } \\
\hline Acquisition mode & single-sided & $\begin{array}{l}\text { Wanted High } \\
\text { Frequency Limit }\end{array}$ & 15798 \\
\hline Resolution & 1 & $\begin{array}{l}\text { Wanted Low } \\
\text { Frequency Limit }\end{array}$ & 0 \\
\hline \multicolumn{4}{|l|}{ FT-Parameter } \\
\hline Apodization Function & $\begin{array}{l}\text { Norton-Beer, Me- } \\
\text { dium }\end{array}$ & Phase Resolution & 128 \\
\hline Zero Filling & 2 & $\begin{array}{l}\text { Phase Correction } \\
\text { Mode }\end{array}$ & Mertz \\
\hline
\end{tabular}




\section{A.5 Temperaturbestimmung}

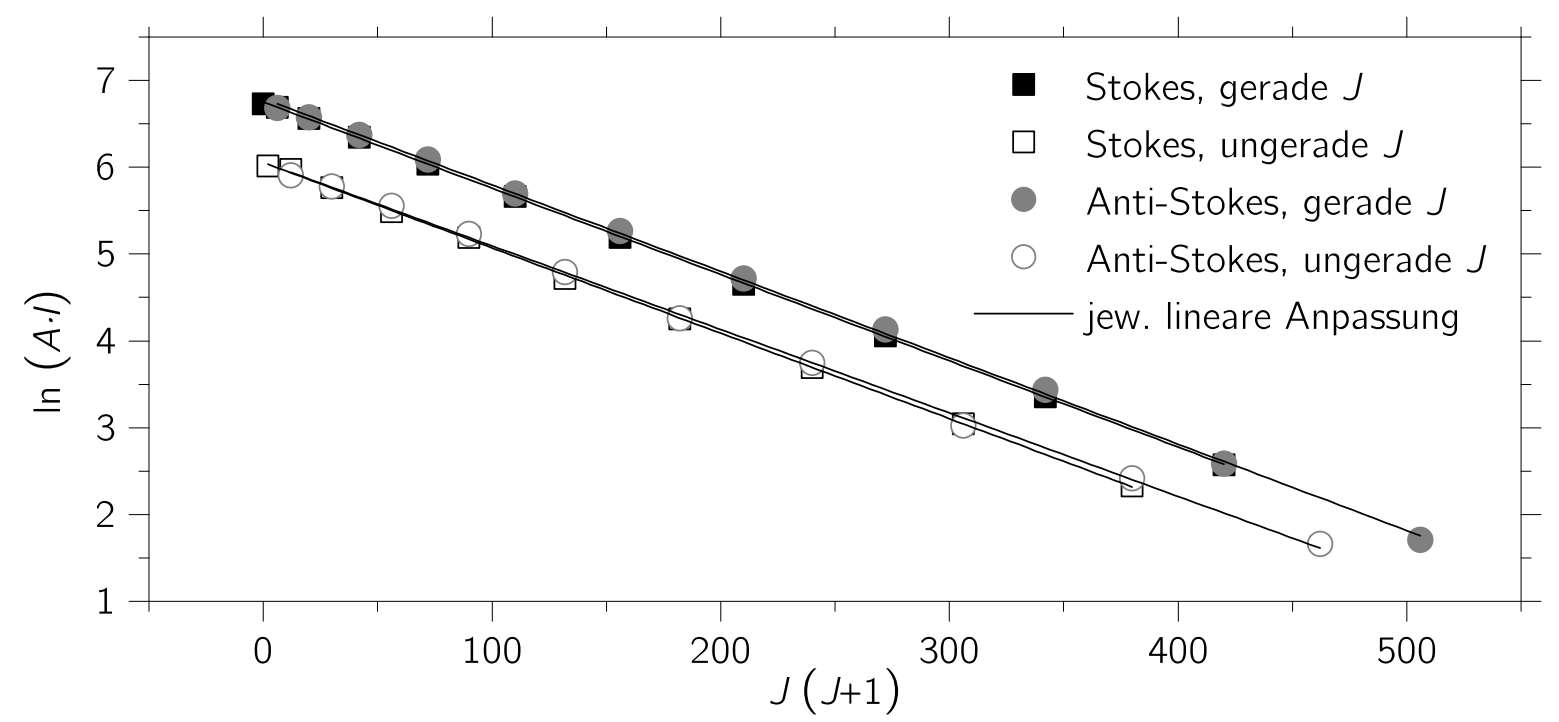

Abbildung A.5.1: Auftragung von $J(J+1)$ gegen $\ln (A \cdot I)$ mit $A=\frac{(J+1)(J+2)}{(2 J+3)}$ für StokesSpektren und $A=\frac{J(J-1)}{(2 J-1)}$ für Anti-Stokes-Spektren beispielhaft aufgeführt für Spektrum (b) aus Abbildung 4.1. Die zugehörigen Steigungen der linearen Regressionen finden sich in Tabelle A.5.1. 


\begin{tabular}{lcccc}
\hline & $m / 10^{-3}$ & $\Delta m / 10^{-3}$ & $T / \mathrm{K}$ & $\Delta T / \mathrm{K}$ \\
\hline mit Polfilter (Spektrum (b) Abb. 4.1) & & & \\
Stokes, gerade $J$ & -9.94 & 0.02 & 288 & 1 \\
Stokes, ungerade $J$ & -9.83 & 0.06 & 291 & 2 \\
Anti-Stokes, gerade $J$ & -9.95 & 0.06 & 288 & 2 \\
Anti-Stokes, ungerade $J$ & -9.60 & 0.10 & 298 & 3 \\
\hline mit Kantenfilter (Spektrum (c) Abb. 4.1) & & & \\
Stokes, gerade $J$ & -9.74 & 0.03 & 294 & 1 \\
Stokes, ungerade $J$ & -9.50 & 0.20 & 301 & 6 \\
Anti-Stokes, gerade $J$ & -9.00 & 0.50 & 320 & 20 \\
Anti-Stokes, ungerade $J$ & -9.20 & 0.30 & 310 & 10 \\
\hline
\end{tabular}

Tabelle A.5.1: Steigung $m$ und aus der Auftragung gegen $J(J+1)$ bestimmte Rotationstemperatur $T_{\text {rot }}$ in K mit jeweiligen Fehlern von Stickstoff für Spektrum (b) und (c) in Abbildung 4.1.

\begin{tabular}{ccccc}
\hline$\widetilde{\nu} / \mathrm{cm}^{-1}$ & $J_{\text {Stokes }}$ & $I_{\text {Anti-Stokes }}$ & $I_{\text {Stokes }}$ & $T_{\text {rot }} / \mathrm{K}$ \\
\hline 11.9 & 0 & 532.1 & 559.4 & 318 \\
20.2 & 1 & 441.2 & 490.3 & 260 \\
27.8 & 2 & 1227.2 & 1383.0 & 312 \\
35.7 & 3 & 720.7 & 872.8 & 253 \\
43.9 & 4 & 1592.8 & 1928.2 & 308 \\
51.8 & 5 & 835.9 & 1033.1 & 326 \\
59.5 & 6 & 1645.5 & 2133.8 & 307 \\
67.9 & 7 & 794.6 & 1027.9 & 350 \\
75.6 & 8 & 1408.1 & 1985.6 & 296 \\
83.4 & 9 & 632.8 & 943.1 & 282 \\
91.2 & 10 & 1108.8 & 1657.0 & 305 \\
99.2 & 11 & 442.1 & 698.8 & 292 \\
107.6 & 12 & 752.9 & 1213.6 & 303 \\
115.4 & 13 & 307.7 & 510.9 & 305 \\
123.1 & 14 & 481.1 & 812.0 & 315 \\
131.0 & 15 & 169.5 & 330.9 & 265 \\
138.8 & 16 & 271.2 & 504.9 & 300 \\
146.7 & 17 & 103.5 & 194.0 & 313 \\
155.1 & 18 & 129.1 & 279.9 & 271 \\
162.8 & 19 & 54.0 & 105.5 & 324 \\
170.6 & 20 & 59.4 & 141.1 & 267 \\
\hline
\end{tabular}

Tabelle A.5.2: Wellenzahlen $\widetilde{\nu}$ in $\mathrm{cm}^{-1}$ und zugehörige Rotationsquantenzahl $J$ des Ausgangsniveaus der Stokes-Rotationsübergänge, relative Intensitäten $I$ der Stokes- und Anti-Stokes-Linien und die über das Intensitätsverhältnis bestimmten Rotationstemperaturen $T_{\text {rot }}$ in $\mathrm{K}$ von Stickstoff für Spektrum (b) in Abbildung 4.1. 


\begin{tabular}{cccccc}
\hline$\widetilde{\nu} / \mathrm{cm}^{-1}$ & $J_{\text {Stokes }}$ & $I_{\text {Anti-Stokes }}$ & $I_{\text {Stokes }}$ & $T_{\text {rot }} / \mathrm{K}$ & $T_{\text {rot }}($ korr $) / \mathrm{K}$ \\
\hline 83.4 & 9 & 1804.4 & 3710.1 & 161 & 172 \\
91.2 & 10 & 3464.8 & 6777.7 & 187 & 201 \\
99.2 & 11 & 1533.5 & 2908.7 & 212 & 229 \\
107.6 & 12 & 2569.2 & 4946.3 & 225 & 242 \\
115.4 & 13 & 1042.8 & 2008.1 & 240 & 258 \\
123.1 & 14 & 1665.7 & 3273.6 & 248 & 266 \\
131.0 & 15 & 640.5 & 1299.3 & 252 & 269 \\
138.8 & 16 & 973.3 & 2045.6 & 254 & 271 \\
146.7 & 17 & 374.2 & 778.9 & 271 & 289 \\
155.1 & 18 & 534.9 & 1153.5 & 273 & 290 \\
162.8 & 19 & 188.5 & 423.7 & 272 & 288 \\
170.6 & 20 & 258.7 & 602.3 & 273 & 289 \\
178.9 & 21 & 87.0 & 207.7 & 278 & 293 \\
186.5 & 22 & 114.2 & 281.9 & 279 & 294 \\
194.7 & 23 & 37.7 & 94.9 & 284 & 299 \\
202.4 & 24 & 47.2 & 124.5 & 282 & 296 \\
210.1 & 25 & 16.0 & 39.8 & 310 & 326 \\
218.4 & 26 & 17.6 & 50.6 & 280 & 292 \\
\hline
\end{tabular}

Tabelle A.5.3: Wellenzahlen $\widetilde{\nu}$ in $\mathrm{cm}^{-1}$ und zugehörige Rotationsquantenzahl $J$ des Ausgangsniveaus der Stokes-Rotationsübergänge, relative Intensitäten $I$ der Stokes- und Anti-Stokes-Linien und die über das Intensitätsverhältnis bestimmten Rotationstemperaturen $T_{\text {rot }}$ in $\mathrm{K}$ von Stickstoff für Spektrum (c) in Abbildung 4.1 sowie die um die Monochromator- und Filterfehler korrigierten Werte $T_{\text {rot }}$ (korr). 


\begin{tabular}{cccccc}
\hline$\widetilde{\nu} / \mathrm{cm}^{-1}$ & $J_{\text {Stokes }}$ & $I_{\text {Anti-Stokes }}$ & $I_{\text {Stokes }}$ & $T_{\text {rot }} / \mathrm{K}$ & $T_{\text {rot }}(\mathrm{korr}) / \mathrm{K}$ \\
\hline \multicolumn{7}{c}{ mit Polfilter } & Spektrum (b) in Abb. 4.3$)$ & & \\
11.9 & 0 & 550.7 & 916.9 & 33 & - \\
20.2 & 1 & 332.3 & 718.2 & 37 & - \\
27.8 & 2 & 615.3 & 1520.0 & 44 & - \\
35.7 & 3 & 159.0 & 625.1 & 37 & - \\
43.9 & 4 & 161.1 & 835.8 & 38 & - \\
51.8 & 5 & 10.8 & 233.8 & 42 & - \\
59.5 & 6 & 27.8 & 221.6 & 41 & - \\
67.9 & 7 & 4.5 & 39.6 & 44 & - \\
\hline mit Kantenfilter & Spektrum (c) in Abb. 4.3) & & \\
67.9 & 7 & - & 13.04 & - & - \\
75.6 & 8 & 1.43 & 7.86 & 63 & 65 \\
83.4 & 9 & 0.21 & 1.03 & 74 & 77 \\
91.2 & 10 & 0.12 & 0.56 & 85 & 88 \\
\hline
\end{tabular}

Tabelle A.5.4: Wellenzahlen $\widetilde{\nu}$ in $\mathrm{cm}^{-1}$ und zugehörige Rotationsquantenzahl $J$ des Ausgangsniveaus der Stokes-Rotationsübergänge, relative Intensitäten $I$ der Stokes- und Anti-Stokes-Linien und die über das Intensitätsverhältnis bestimmten Rotationstemperaturen $T_{\text {rot }}$ in $\mathrm{K}$ von Stickstoff für Spektren (b) und (c) in Abbildung 4.3 sowie für Spektrum (c) die um die Monochromator- und Filterfehler korrigierten Werte $T_{\text {rot }}$ (korr).

\begin{tabular}{|c|c|c|c|c|}
\hline & $m / 10^{-}$ & $\Delta m / 10^{-2}$ & $T / \mathrm{K}$ & $\Delta T / \mathrm{K}$ \\
\hline \multicolumn{5}{|c|}{ mit Polfilter (Spektrum (b) Abb. 4.3) } \\
\hline Stokes, gerade $J$ & -7.49 & 0.02 & 38.2 & 0.1 \\
\hline Stokes, ungerade $J$ & -7.70 & 0.06 & 37.2 & 0.3 \\
\hline Anti-Stokes, gerade $J$ & -7.30 & 0.20 & 40 & 1 \\
\hline Anti-Stokes, ungerade $J$ & -7.10 & 0.10 & 40.3 & 0.6 \\
\hline \multicolumn{5}{|c|}{ mit Kantenfilter (Spektrum (c) Abb. 4.3) } \\
\hline Stokes, gerade $J$ & -7.46 & - & 38 & - \\
\hline Stokes, ungerade $J$ & -8.07 & - & 35 & - \\
\hline Anti-Stokes, gerade $J$ & -5.76 & - & 50 & - \\
\hline Anti-Stokes, ungerade $J$ & - & - & - & - \\
\hline
\end{tabular}

Tabelle A.5.5: Steigung $m$ und aus der Auftragung gegen $J(J+1)$ bestimmte Rotationstemperatur $T_{\text {rot }}$ in K von Stickstoff für Spektren (b) und (c) in Abbildung 4.3. Die jeweiligen Fehler ergeben sich aus der linearen Regression ergeben und konnten für Spektrum (c) nicht ermittelt werden, da hier nur 2 Datenpunkte pro Auftragung ausgewertet werden konnten. 


\begin{tabular}{ccccc}
\hline$d / \mathrm{mm}$ & $\begin{array}{c}\bar{T}_{\text {rot }} / \mathrm{K} \\
\text { (erste } 5 \text { Übergänge) }\end{array}$ & $\begin{array}{c}\text { Anzahl Übergänge } \\
(4-5)\end{array}$ & $\begin{array}{c}\bar{T}_{\text {rot }} / \mathrm{K} \\
\text { (gesamt) }\end{array}$ & Anzahl Übergänge \\
\hline 1 & 38 & 5 & 40 & 8 \\
2 & 23 & 5 & 23 & 5 \\
3 & 19 & 5 & 19 & 5 \\
4 & 15 & 4 & 15 & 4 \\
5 & 15 & 5 & 15 & 4 \\
6 & 29 & 5 & 42 & 7 \\
7 & 43 & 5 & 58 & 9 \\
8 & 61 & 5 & 87 & 11 \\
9 & 102 & 5 & 121 & 11 \\
\hline
\end{tabular}

Tabelle A.5.6: Mittelwerte der über das Stokes-Anti-Stokes-Intensitätsverhältnis bestimmten Rotationstemperaturen für Jetexpansionen von Stickstoff sowie die jeweilige Anzahl an in den Mittelwert einbezogenen Übergänge. (vgl. Abb. 4.4 (a), rote Linie: $\bar{T}_{\text {rot }}$ (erste 5 Übergänge) und rot-gestrichelte Linie: $\bar{T}_{\text {rot }}$ (alle auswertbaren Übergänge))

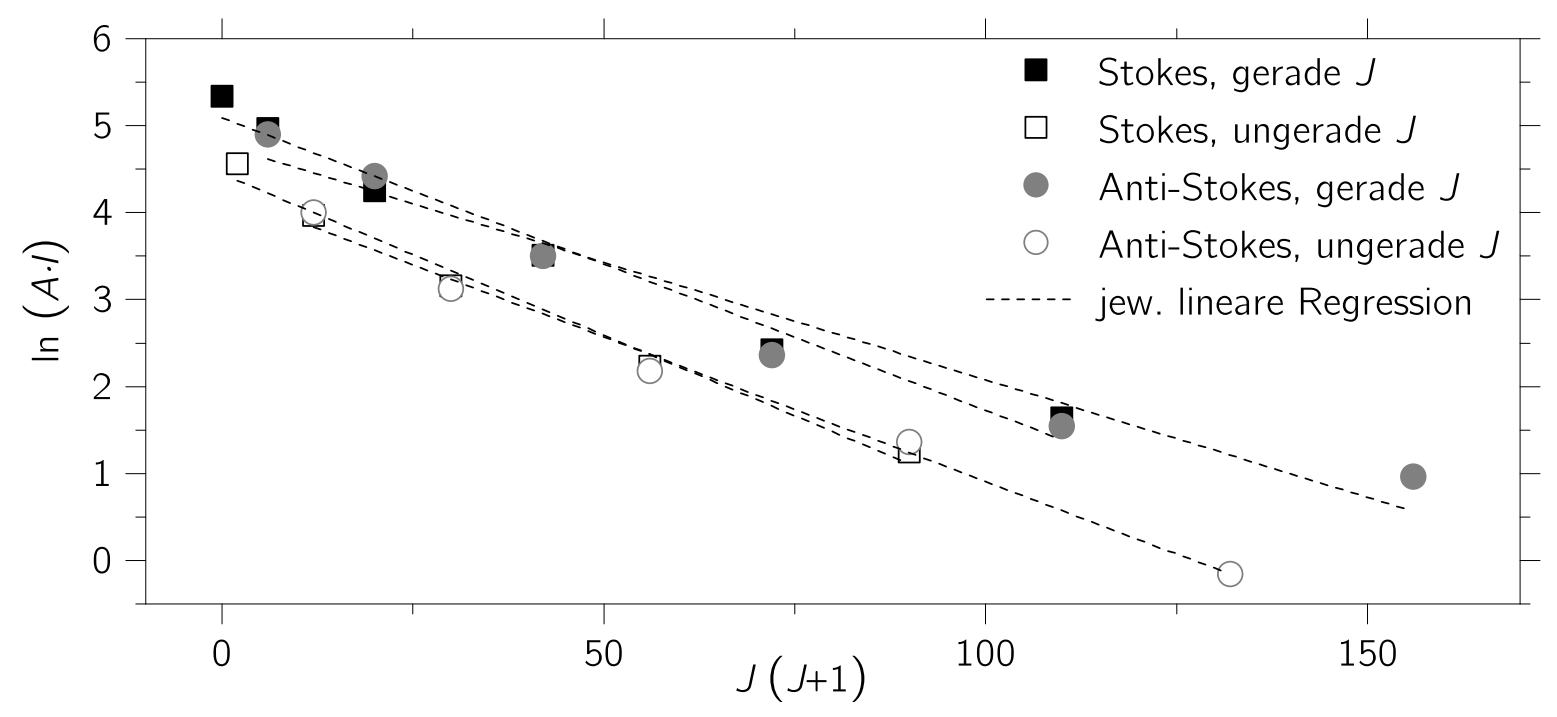

Abbildung A.5.2: Auftragung von $J(J+1)$ gegen $\ln (A \cdot I)$ mit $A=\frac{(J+1)(J+2)}{(2 J+3)}$ für StokesSpektren und $A=\frac{J(J-1)}{(2 J-1)}$ für Anti-Stokes-Spektren beispielhaft aufgeführt für ein Jetspektrum, das bei einem Abstand von $8 \mathrm{~mm}$ zur Düse aufgenommen wurde. Die Abweichungen vom linearen Verhalten, die sich in einem „Abknicken“ ab $J=8$ zeigen, sind wahrscheinlich auf die Heterogenität der Expansion zurückzuführen. 


\section{Referenz-Raman-Spektrometer (PCF-Praktikum, IPC, Georg-August Uni- versität Göttingen):}

Das für Vergleichsmessungen genutzte Raman-Spektrometer erfasst das gestreute Licht ebenfalls im $90^{\circ}$-Winkel. Es wird ein grüner Dauerstrichlaser mit einer Maximalleistung von $5 \mathrm{~W}$ verwendet, der mittels einer Sammellinse $(f=50 \mathrm{~mm})$ fokussiert wird. Für das Experiment ist eine Leistung von $1 \mathrm{~W}$ allerdings ausreichend. Das im Fokuspunkt des Lasers gestreute Licht wird zunächst durch eine Sammellinse $(f=150 \mathrm{~mm})$ kollimiert und durch eine weitere Sammellinse $(f=500 \mathrm{~mm})$ auf den Eintrittsspalt des Monochromators fokussiert. Die Detektion erfolgt über eine durch ein Peltierelement auf $-30^{\circ} \mathrm{C}$ gekühlte CCD-Kamera. Die Spezifikationen der Komponenten sind in Tabelle A.5.7 zusammengefasst.

Zur Kalibrierung wird die Position der Rayleighlinie auf dem CCD-Chip bestimmt und über die folgende Kalibrierfunktion werden die Pixelwerte $(\lambda / \mathrm{px})$ in Wellenzahlen (RamanShift) umgerechnet.

\begin{tabular}{ll} 
RamanShift $/ \mathrm{cm}^{-1}=$ & $0.1845 \cdot\left(\lambda / \mathrm{px}-\lambda_{\text {Rayleigh }} / \mathrm{px}\right)$ \\
\hline Laser: & Coherent Verdi $5,532 \mathrm{~nm}$ \\
Monochromator: & Jobin-Yvon THR 1500 \\
& Brennweite $1.5 \mathrm{~m}$ \\
& Gitter 2400 Linien $/ \mathrm{mm}$ \\
& Auflösung 0.01 nm \\
& Andor Technology \\
CCD-Chip: & Model DU420A-OE \\
& $1024 \times 265$ Pixel \\
\hline
\end{tabular}

Tabelle A.5.7: Experimentelle Komponenten des Raman-Spektrometers, mit dem höher aufgelöste Referenzspektren von Luft aufgenommen wurden. 


\begin{tabular}{|c|c|c|c|c|c|c|}
\hline$\widetilde{\nu} / \mathrm{cm}^{-1}$ & $I_{\text {Anti-Stokes }}$ & $I_{\text {Stokes }}$ & Übergang & $\vartheta_{\mathrm{D}} /{ }^{\circ} \mathrm{C}$ & $T_{\text {rot }} / \mathrm{K}$ & $T_{\text {rot }}($ korr $) / \mathrm{K}$ \\
\hline \multirow[t]{5}{*}{70} & 1.190 & 16.828 & $(202) \leftarrow(000)$ & 25 & 38 & 39 \\
\hline & 0.833 & 15.724 & & 25 & 34 & 35 \\
\hline & 0.583 & 16.295 & & 25 & 30 & 30 \\
\hline & 1.604 & 18.655 & & 80 & 41 & 42 \\
\hline & 0.959 & 14.175 & & 120 & 37 & 38 \\
\hline \multirow[t]{5}{*}{94} & 0.496 & 5.060 & $(312) \leftarrow(212)$ & 25 & 57 & 58 \\
\hline & 0.323 & 5.706 & & 25 & 47 & 47 \\
\hline & 0.236 & 4.692 & & 25 & 45 & 46 \\
\hline & 0.701 & 6.617 & & 80 & 59 & 61 \\
\hline & 0.325 & 4.175 & & 120 & 52 & 53 \\
\hline \multirow[t]{5}{*}{105} & 0.145 & 3.074 & $(313) \leftarrow(111)$ & 25 & 49 & 50 \\
\hline & 0.093 & 3.339 & & 25 & 42 & 43 \\
\hline & 0.078 & 2.923 & & 25 & 42 & 42 \\
\hline & 0.180 & 3.780 & & 80 & 49 & 50 \\
\hline & 0.127 & 2.742 & & 120 & 59 & 50 \\
\hline \multirow[t]{5}{*}{113} & 1.388 & 44.888 & $(303) \leftarrow(101)$ & 25 & 46 & 47 \\
\hline & 0.706 & 45.999 & & 25 & 39 & 39 \\
\hline & 0.502 & 45.393 & & 25 & 36 & 36 \\
\hline & 1.550 & 54.950 & & 80 & 45 & 46 \\
\hline & 0.675 & 40.179 & & 120 & 40 & 40 \\
\hline \multirow[t]{5}{*}{131} & 0.390 & 14.736 & $(312) \leftarrow(110)$ & 25 & 51 & 52 \\
\hline & 0.219 & 15.480 & & 25 & 44 & 44 \\
\hline & 0.114 & 14.700 & & 25 & 39 & 39 \\
\hline & 0.357 & 18.648 & & 80 & 47 & 48 \\
\hline & 0.127 & 13.161 & & 120 & 40 & 41 \\
\hline \multirow[t]{5}{*}{136} & 0.063 & 9.385 & $(220) \leftarrow(000)$ & 25 & 39 & 39 \\
\hline & 0.127 & 9.371 & & 25 & 45 & 46 \\
\hline & 0.039 & 9.164 & & 25 & 36 & 36 \\
\hline & 0.171 & 10.643 & & 80 & 47 & 48 \\
\hline & 0.128 & 7.478 & & 120 & 48 & 48 \\
\hline \multirow[t]{5}{*}{189} & 0.162 & 19.499 & $(321) \leftarrow(101)$ & 25 & 56 & 57 \\
\hline & 0.090 & 19.500 & & 25 & 50 & 50 \\
\hline & 0.040 & 19.662 & & 25 & 43 & 44 \\
\hline & 0.185 & 23.627 & & 80 & 55 & 56 \\
\hline & 0.112 & 17.182 & & 120 & 53 & 54 \\
\hline
\end{tabular}

Tabelle A.5.8: Rotationstemperaturen von Wasser: Wellenzahl $\widetilde{\nu}$ in $\mathrm{cm}^{-1}$, relative Linienintensität $I$ für Stokes- und Anti-Stokes-Übergänge, Rotationsquantenzahlen $\left(J K_{\mathrm{a}} K_{\mathrm{c}}\right)$ des Ausgangs- und Endniveaus, Düsentemperatur $\vartheta_{\mathrm{D}}$ in ${ }^{\circ} \mathrm{C}$ und effektive Rotationstemperaturen $T_{\text {rot }}$ in K, sowie die um Filter- und Monochromatorfehler korrigierte Temperatur $T_{\text {rot }}($ korr $)$ in K. Die drei Werte für $\vartheta_{\mathrm{D}}=25^{\circ} \mathrm{C}$ sind unabhängige Messergebnisse 


\begin{tabular}{cccccc}
\hline$\widetilde{\nu} / \mathrm{cm}^{-1}$ & $I_{\text {Anti-Stokes }}$ & $I_{\text {Stokes }}$ & $\vartheta_{\mathrm{D}} /{ }^{\circ} \mathrm{C}$ & $T_{\text {vib }} / \mathrm{K}$ & $T_{\text {vib }}(\mathrm{korr}) / \mathrm{K}$ \\
\hline \multirow{2}{*}{116} & 0.223 & 16.649 & 25 & 38 & 39 \\
& 0.696 & 17.468 & 80 & 51 & 52 \\
& 0.631 & 18.230 & 150 & 49 & 50 \\
\hline \multirow{2}{*}{80} & 0.177 & 29.507 & 25 & 77 & 78 \\
& 0.730 & 35.345 & 80 & 101 & 103 \\
& 0.977 & 33.716 & 150 & 111 & 112 \\
\hline 364 & 0.059 & 22.409 & 25 & 86 & 87 \\
& 0.282 & 26.453 & 80 & 112 & 114 \\
& 0.195 & 22.732 & 150 & 107 & 109 \\
\hline 416 & 0.235 & 89.315 & 25 & 99 & 99 \\
& 0.634 & 87.548 & 80 & 118 & 119 \\
& 0.569 & 88.758 & 150 & 116 & 117 \\
\hline
\end{tabular}

Tabelle A.5.9: Schwingungstemperaturen von 2,2,2-Trifluorethanol: Wellenzahl $\widetilde{\nu}$ in $\mathrm{cm}^{-1}$, relative Bandenintensitäten $I$ für Stokes- und Anti-Stokes-Übergänge, Düsentemperatur $\vartheta_{\mathrm{D}}$ in ${ }^{\circ} \mathrm{C}$ und effektive Schwingungstemperaturen $T_{\text {vib }}$ in $\mathrm{K}$, sowie die um Filter- und Monochromatorfehler korrigierte Temperatur $T_{\text {vib }}($ korr $)$ in $\mathrm{K}$.

\begin{tabular}{llllll}
\hline \multicolumn{7}{c}{ Theoret. $I_{\mathrm{AS} / \mathrm{S}}$ für eine $T_{\text {vib }}$ von } \\
$\widetilde{\nu} / \mathrm{cm}^{-1}$ & $50 \mathrm{~K}$ & $70 \mathrm{~K}$ & $80 \mathrm{~K}$ & $110 \mathrm{~K}$ & $120 \mathrm{~K}$ \\
\hline 116 & $\mathbf{0 . 0 4}$ & 0.1 & 0.1 & 0.2 & 0.3 \\
218 & 0.002 & 0.01 & $\mathbf{0 . 0 2}$ & 0.06 & 0.08 \\
283 & 0.0003 & 0.003 & 0.007 & $\mathbf{0 . 0 3}$ & 0.04 \\
364 & 0.00003 & 0.0006 & 0.002 & $\mathbf{0 . 0 1}$ & 0.01 \\
416 & 0.000007 & 0.0002 & 0.0006 & 0.005 & $\mathbf{0 . 0 0 8}$ \\
\hline
\end{tabular}

Tabelle A.5.10: Theoretische Anti-Stokes/Stokes-Intensitätsverhältnisse $\left(I_{\mathrm{AS} / \mathrm{S}}\right)$ für verschiedene Schwingungstemperaturen und die niederfrequenten Schwingungsbanden von 2,2,2-Trifluorethanol. Die für Spektrum (c) in Abbildung 4.9 als Dämpfungsfaktoren verwendeten Werte sind in fett gedruckt. 


\section{A.6 Wassercluster}

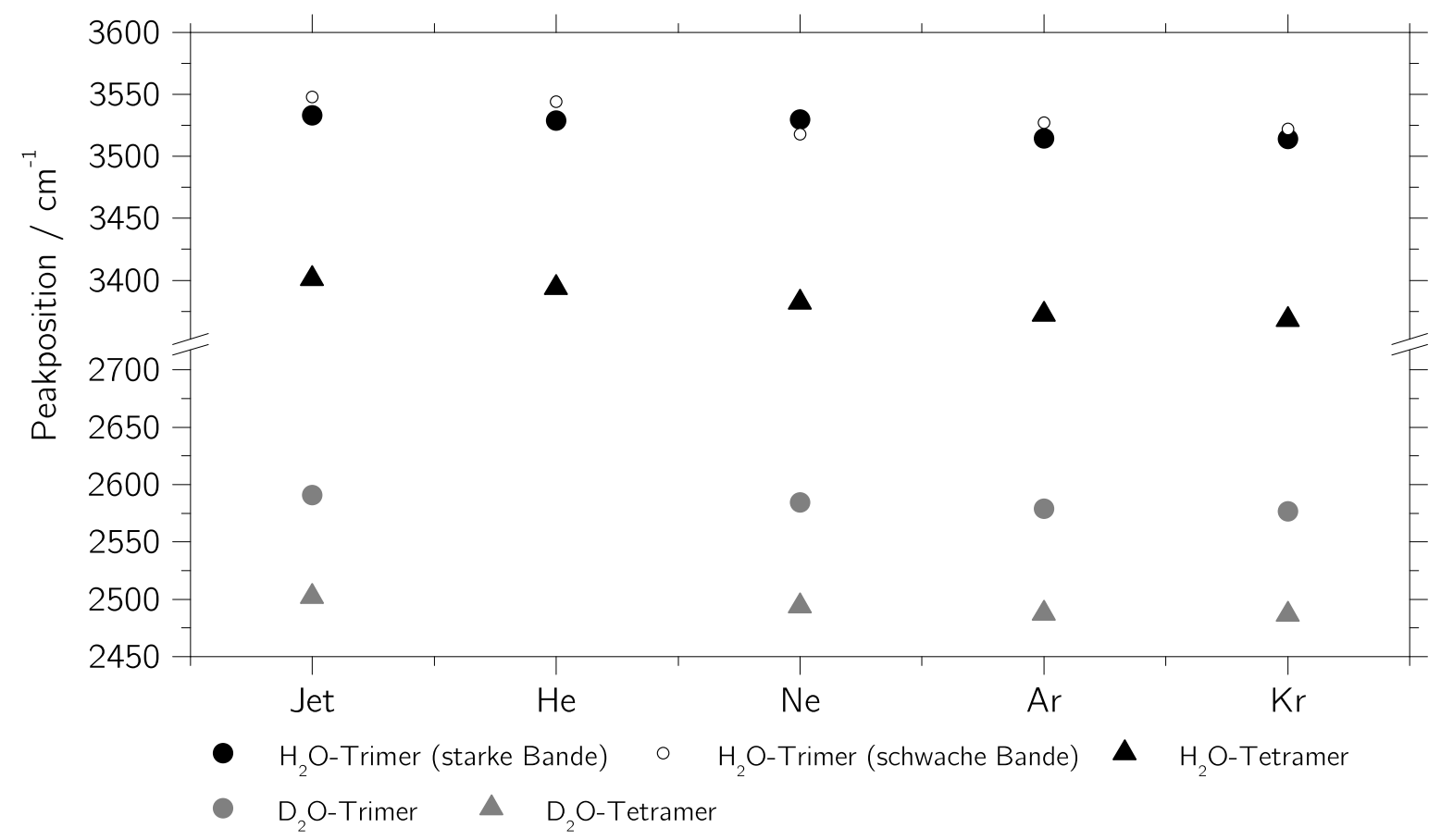

Abbildung A.6.1: Peakpositionen der IR-Trimer- und -Tetramerbanden in verschiedenen Umgebungen. Die Peakpositionen von Trimer und Tetramer verschieben sich linear zu niedrigerer Wellenzahl beim Übergang von der Gasphase ${ }^{21}$ über He-Tröpfchen ${ }^{110}$ zu Ne-, Ar- und Kr-Matrizen ${ }^{111}$ sowohl für $\mathrm{H}_{2} \mathrm{O}$ als auch für $\mathrm{D}_{2} \mathrm{O}$. Besonders auffällig ist, dass die Schulter, die für das $\mathrm{H}_{2} \mathrm{O}$-Trimer gefunden wurde, außer in NeMatrixspektren grundsätzlich blauverschoben zum Hauptpeak auftaucht. 


\begin{tabular}{ccc}
\hline$\widetilde{\nu} / \mathrm{cm}^{-1}$ & Zuordnung & $\begin{array}{c}\text { Übergang } \\
\left(J^{\prime} K_{a}^{\prime} K_{c}^{\prime}\right) \leftarrow\left(J K_{a} K_{c}\right)\end{array}$ \\
\hline 1561 & 1 & $(110) \leftarrow(212)$ \\
1595 & 1 & $(n 0 n) \leftarrow(n 0 n)$ \\
1598 & 1 & $(212) \leftarrow(212) ;(111) \leftarrow(111)$ \\
& & $(110) \leftarrow(110) ;(211) \leftarrow(211)$ \\
1636 & 1 & $(212) \leftarrow(110)$ \\
1645 & 1 & $(313) \leftarrow(211)$ \\
1666 & 1 & $(202) \leftarrow(000)$ \\
1675 & 1 & $(313) \leftarrow(111)$ \\
1703 & 1 & $(303) \leftarrow(101)$ \\
1709 & 1 & $(221) \leftarrow(101)$ \\
1719 & 1 & $(40) \leftarrow(110)$ \\
1732 & 1 & $(321) \leftarrow(101)$ \\
1744 & 1 & $(330) \leftarrow(212)$ \\
1748 & 1 & $(330) \leftarrow(110)$ \\
1796 & 1 & $(331) \leftarrow(111)$ \\
1830 & 1 & \\
1867 & 1 & \\
1871 & 1 &
\end{tabular}

Tabelle A.6.1: Zuordnung der OH-Knickschwingungsübergänge des Wasser-Monomers in Abbildung 5.4 gemäß Ref. 121. Für die Monomerübergänge sind die Rotationsquantenzahlen $\left(J, K_{a}, K_{c}\right)$ des Ausgangs- und Endniveaus angegeben. Außerdem vorsichtige Zuordnung eines Dimerpeaks (2) sowie eines Bereichs, in dem größere Cluster $(>2)$ auftreten. 


\section{A.7 Isomerisierungen}

\section{A.7.1 2,2,2-Trifluorethanol}

\begin{tabular}{|c|c|c|c|c|c|c|}
\hline \multicolumn{2}{|c|}{ Experiment } & \multirow{3}{*}{$\begin{array}{c}\text { B3LYP/aVTZ } \\
\text { harmonisch } \\
\omega_{i}\left(2 E_{0, i}\right)\end{array}$} & \multicolumn{2}{|c|}{ B2PLYP-D3-BJ/def2-TZVP } & \multicolumn{2}{|c|}{ MP2/aVTZ } \\
\hline & & & harmonisch & $\begin{array}{l}\text { anharm. } \\
\text { (VPT2) }\end{array}$ & harmonisch & $\begin{array}{l}\text { anharm. } \\
\text { (VPT2) }\end{array}$ \\
\hline$\widetilde{\nu}_{i}$ & $\widetilde{\nu}_{i}^{\text {entstört }}$ & & $\omega_{i}\left(2 E_{0, i}\right)$ & $\widetilde{\nu}_{i}$ & $\omega_{i}\left(2 E_{0, i}\right)$ & $\widetilde{\nu}_{i}$ \\
\hline 3657 & & $3806(3769)$ & $3811(3772)$ & 3635 & $3831(3787)$ & 3655 \\
\hline 3002 & & $3115(3087)$ & $3150(3113)$ & 3010 & $3182(3137)$ & 3045 \\
\hline 2948 & 2937 & $3031(3007)$ & $3060(3029)$ & 2896 & $3092(3053)$ & 2992 \\
\hline 2897 & 2921 & & & 2970 & & 2907 \\
\hline 1458 & & $1489(1481)$ & $1505(1494)$ & 1469 & $1510(1497)$ & 1469 \\
\hline 1415 (IR) & & $1430(1426)$ & $1450(1441)$ & 1412 & $1453(1443)$ & 1414 \\
\hline 1366 & & $1387(1382)$ & $1399(1391)$ & 1363 & $1398(1390)$ & 1363 \\
\hline 1293 & & $1280(1283)^{*}$ & $1307(1303)$ & 1277 & $1320(1313)$ & 1289 \\
\hline 1264 & & $1261(1261)$ & $1279(1275)$ & 1250 & $1284(1279)$ & 1255 \\
\hline 1183 & & $1152(1160)^{*}$ & $1183(1183)$ & 1156 & $1197(1194)$ & 1170 \\
\hline 1142 & & $1134(1136)^{*}$ & $1155(1152)$ & 1131 & 1162 (1157) & 1137 \\
\hline 1088 & & $1090(1089)$ & 1105 (1101) & 1078 & 1112 (1106) & 1084 \\
\hline 940 & & 938 (938) & $955(951)$ & 937 & $958(953)$ & 939 \\
\hline 835 & 833 & $823(825)^{*}$ & $834(834)$ & 819 & $844(841)$ & 833 \\
\hline 828 & 831 & & & 823 & & 821 \\
\hline 665 & & $657(659)^{*}$ & $666(665)$ & 659 & $668(667)$ & 661 \\
\hline 547 & & $538(540)^{*}$ & $547(547)$ & 542 & $549(549)$ & 544 \\
\hline 541 & & $527(530)^{*}$ & $534(536)^{*}$ & 531 & $534(535)^{*}$ & 528 \\
\hline 416 & & $413(413)$ & 419 (418) & 411 & $420(419)$ & 412 \\
\hline 364 & & $371(370)$ & $383(378)$ & 366 & $377(374)$ & 363 \\
\hline 283 & & $301(297)$ & $317(308)$ & 302 & $308(302)$ & 290 \\
\hline 235 & 227 & & & 237 & & 236 \\
\hline 218 & 221 & 219 (219) & $224(223)$ & 220 & $222(222)$ & 221 \\
\hline 116 & & $113(114)^{*}$ & 117 (117) & 119 & $120(119)$ & 117 \\
\hline $\mathrm{ZPE} / \mathrm{k}$ & $\mathrm{mol}^{-1}$ & $150.0(149.5)$ & $151.9(150.9)$ & 150.1 & $152.8(151.6)$ & 151.0 \\
\hline
\end{tabular}

Tabelle A.7.1: Experimentelle und ggf. entstörte Bandenpositionen von 2,2,2Trifluorethanol im Vergleich mit harmonischen Frequenzen $\left(\omega_{i}\right)$ sowie den nach Gleichung 6.1 korrigierten Nullpunktsenergiebeiträgen $\left(E_{0, i}\right)$ und im Vergleich mit anharmonischen quantenchemischen Rechnungen jeweils in $\mathrm{cm}^{-1}$. Um die Auswirkung der Korrektur besser sichtbar zu machen ist jeweils der doppelte Nullpunktsenergiebeitrag $\left(2 E_{0, i}\right)$ angegeben. Außerdem sind die berechneten experimentell korrigierten Nullpunktsenergien (ZPE) in $\mathrm{kJ} / \mathrm{mol}$ angegeben. Diejenigen experimentell korrigierten Banden, deren Wellenzahlwerte größer als die harmonischen Werte sind, sind mit einem * markiert. Es zeigt sich, dass die Korrektur für B3LYP nicht unbedingt aussagekräftig ist und die Nullpunktsenergie unterschätzt wird, während sich für die beiden anderen Methoden ein konsistentes Bild ergibt. 


\section{A.7.2 3,3,3-Trifluorpropanol}

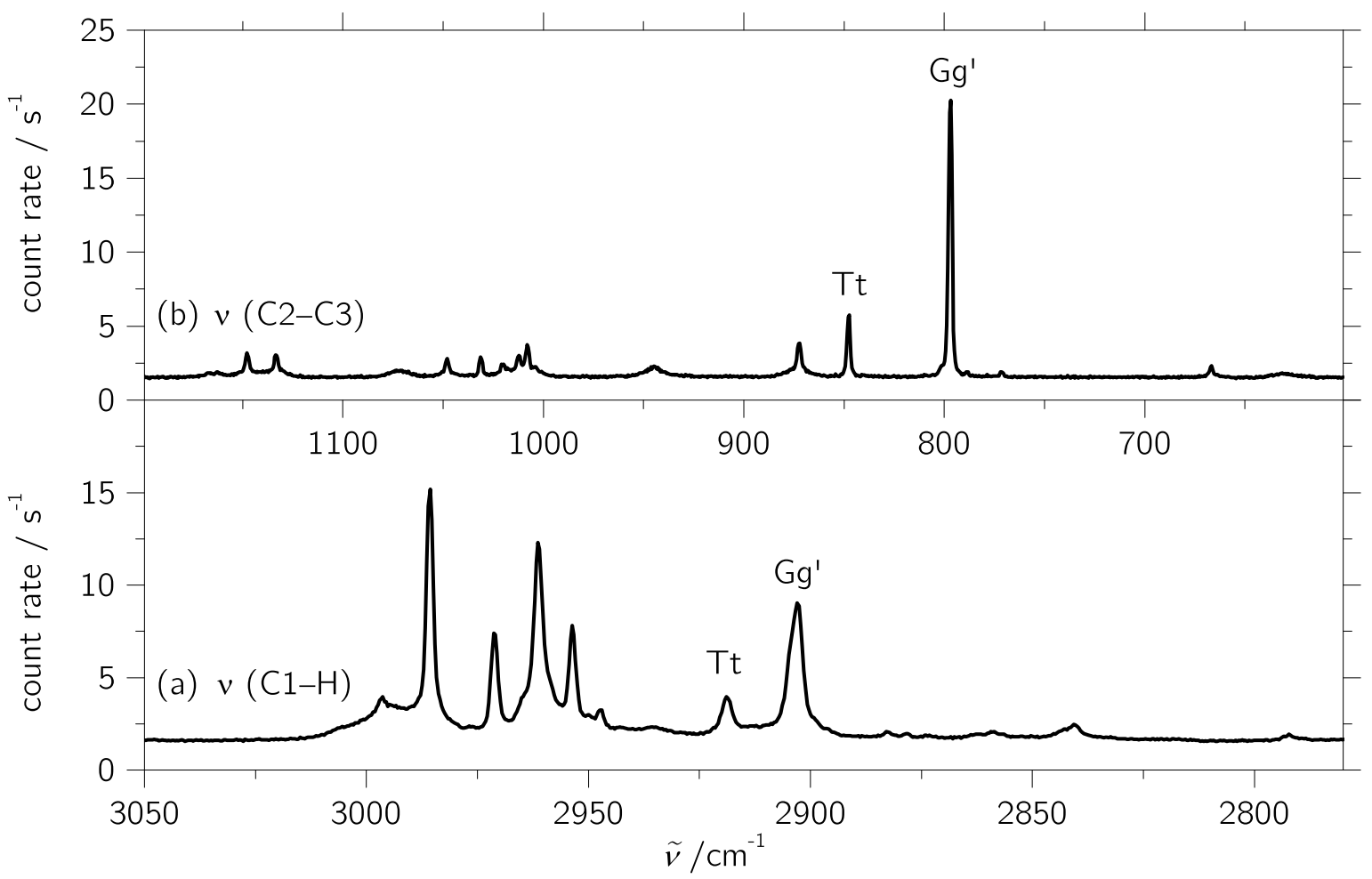

Abbildung A.7.1: Spektren von 3,3,3-Trifluorpropanol (a) im CH-Streckschwingungsbereich und (b) im CC-Streckschwingungsbereich. Die mit Tt und $\mathrm{Gg}^{\prime}$ markierten Banden wurden zur Abschätzung des Energieunterschiedes der beiden Konformere genutzt. Die Zuordnung der weiteren Banden ist in Ref. 18 aufgeführt. Details zu den Messbedingungen finden sich im Anhang A.3. 


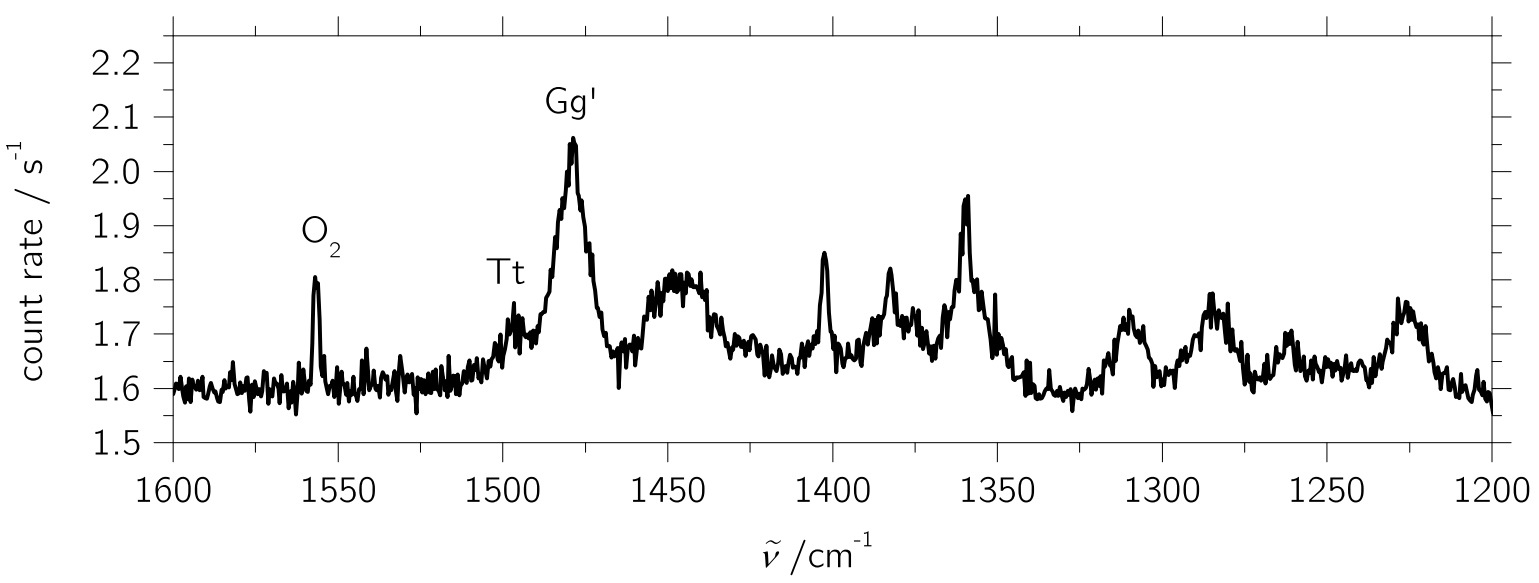

Abbildung A.7.2: Spektrum von 3,3,3-Trifluorpropanol im Bereich von $1600-1200 \mathrm{~cm}^{-1}$. Die zwei CH-Knickschwingungsbanden, deren Obertöne besonders für eine Fermiresonanz mit den CH-Streckschwingungen infrage kämen, sind mit Tt und $\mathrm{Gg}^{\prime}$ markiert. Bei $1556 \mathrm{~cm}^{-1}$ ist außerdem eine geringe Verunreinigung mit Luftsauerstoff $\mathrm{zu}$ erkennen. Details zu den Messbedingungen finden sich im Anhang A.3.

\begin{tabular}{|c|c|c|c|c|c|c|c|}
\hline & $\begin{array}{l}\text { Lorentz- } \\
\text { fit }\end{array}$ & $\begin{array}{l}\text { Höhe } \times \\
\text { FWHM }\end{array}$ & $\begin{array}{l}\ln (F) \\
\text { Integration } \\
(-10 \% \text { Sockel })\end{array}$ & $\begin{array}{c}\text { Integration } \\
(3 \times \text { FWHM })\end{array}$ & $\ln (\bar{F})$ & $F$ & $C$ \\
\hline $\begin{array}{c}\mathrm{C} 2-\mathrm{C} 3 \\
\vartheta_{\mathrm{D}} /{ }^{\circ} \mathrm{C}\end{array}$ & & & & & & & \\
\hline $\begin{array}{c}25 \\
80 \\
150\end{array}$ & $\begin{array}{l}-1.4786 \\
-1.4522 \\
-1.3196\end{array}$ & $\begin{array}{l}-1.4989 \\
-1.4745 \\
-1.3233\end{array}$ & $\begin{array}{l}-1.7631 \\
-1.6513 \\
-1.5847\end{array}$ & $\begin{array}{l}-1.5636 \\
-1.5360 \\
-1.3761\end{array}$ & $\begin{array}{l}-1.6(1) \\
-1.53(7) \\
-1.4(1)\end{array}$ & $\begin{array}{l}0.21(2) \\
0.22(2) \\
0.25(3)\end{array}$ & $0.723(4)$ \\
\hline $\begin{array}{l}\mathrm{C} 1-\mathrm{H} \\
\vartheta_{\mathrm{D}} /{ }^{\circ} \mathrm{C}\end{array}$ & & & & & & & \\
\hline $\begin{array}{c}25 \\
80 \\
150\end{array}$ & $\begin{array}{l}-1.6390 \\
-1.5068 \\
-1.4635\end{array}$ & $\begin{array}{l}-1.5561 \\
-1.4940 \\
-1.4479\end{array}$ & $\begin{array}{l}-1.7787 \\
-1.6569 \\
-1.6204\end{array}$ & $\begin{array}{l}-1.5692 \\
-1.5137 \\
-1.3598\end{array}$ & $\begin{array}{l}-1.64(9) \\
-1.54(6) \\
-1.47(9)\end{array}$ & $\begin{array}{l}0.19(2) \\
0.21(1) \\
0.23(2)\end{array}$ & $1.06(2)$ \\
\hline $\begin{array}{c}\mathrm{O}-\mathrm{H} \\
\vartheta_{\mathrm{D}} /{ }^{\circ} \mathrm{C} \\
25 \\
80 \\
150\end{array}$ & $\begin{array}{l}-0.6226 \\
-0.5845 \\
-0.3714\end{array}$ & $\begin{array}{l}-0.5281 \\
-0.4705 \\
-0.2615\end{array}$ & $\begin{array}{l}-0.8970 \\
-0.8248 \\
-0.5704\end{array}$ & $\begin{array}{l}-0.7018 \\
-0.7093 \\
-0.4291\end{array}$ & $\begin{array}{l}-0.7(1) \\
-0.6(1) \\
-0.4(1)\end{array}$ & $\begin{array}{l}0.50(7) \\
0.52(7) \\
0.66(7)\end{array}$ & $2.16(3)$ \\
\hline
\end{tabular}

Tabelle A.7.2: Mittels verschiedener Methoden bestimmte logarithmierte Intensitätsverhältnisse $\ln (F)$ sowie deren Mittelwert $\ln (\bar{F})$ und die für die Auftragung verwendeten mittleren Werte von $F$ und dem berechneten Verhältnis der Streuquerschnitte $C{ }^{18}$ Die Fehlergrenzen sind in Klammern angegeben. Die absoluten $\ln (F)$-Werte können recht stark voneinander abweichen, da die aus den Auftragungen bestimmten $\Delta H^{\ominus}$-Werte jedoch im jeweiligen Spektralbereich sehr ähnlich sind, ist die Verwendung der Mittelwerte gerechtfertigt. 


\section{A.7.3 NMF}

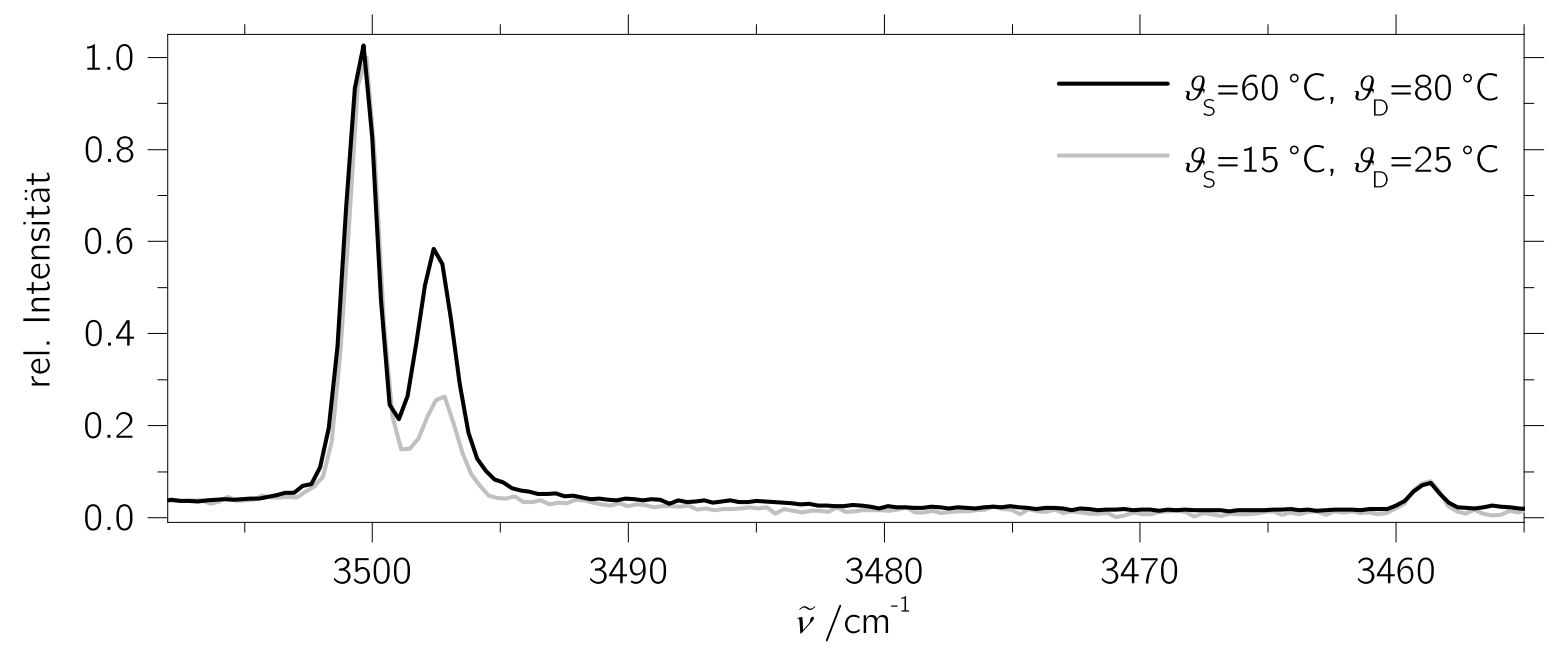

Abbildung A.7.3: Raman-Jetspektren von NMF für eine hohe (schwarz) und eine niedrige Konzentration (hellgrau), die auf die NH-Streckschwingungsfundamentale des transMonomers normiert sind. Im hochkonzentrierten Spektrum kommt es vermehrt zu Clusterbildung, wodurch die Monomertemperatur ansteigt, da die Kondensationswärme abgeführt werden muss. Die Bande bei $3497 \mathrm{~cm}^{-1}$ steigt vom niedrig- zum hochkonzentrierten Jetspektrum stark an und ist somit eine nützliche Sonde für die trans-Monomerschwingungstemperatur. Details zu den Messbedingungen finden sich im Anhang A.3. 


\begin{tabular}{lcccc}
\hline & $\begin{array}{c}\text { B3LYP/ } \\
6-311++\mathrm{G}(\mathrm{d}, \mathrm{p})\end{array}$ & $\begin{array}{c}\text { B3LYP/ } \\
\text { aVTZ }\end{array}$ & $\begin{array}{c}\text { MP2/ } \\
\text { aVTZ }\end{array}$ & \\
\hline$\sigma^{\prime}(t) / 10^{-35} \mathrm{~m}^{2} / \mathrm{sr}$ & 10.92 & 10.76 & 9.71 & \\
$\sigma^{\prime}(c) / 10^{-35} \mathrm{~m}^{2} / \mathrm{sr}$ & 9.59 & 9.21 & 8.24 & \\
$C_{\mathrm{c} / \mathrm{t}}$ & 0.88 & 0.86 & 0.85 & \\
$\bar{C}_{\mathrm{c} / \mathrm{t}}$ & & & & $0.86(1)$ \\
$\sigma^{\prime}(t t) / 10^{-35} \mathrm{~m}^{2} / \mathrm{sr}$ & 35.41 & 36.33 & - & \\
$\sigma^{\prime}(c c) / 10^{-35} \mathrm{~m}^{2} / \mathrm{sr}$ & 54.42 & 53.75 & - & \\
$C_{\mathrm{cc} / \mathrm{tt}}$ & 1.54 & 1.48 & - & \\
$\bar{C}_{\mathrm{cc} / \mathrm{tt}}$ & & & & 1.51 \\
\hline
\end{tabular}

Tabelle A.7.3: Auf verschiedenen Niveaus berechnete differentielle Streuquerschnitte $\sigma^{\prime}$ für die NH-Streckschwingung von cis- (c) und trans-NMF (t), sowie für die Streckschwingung der gebundenen NH-Gruppe im trans-trans- (tt) und die stark Raman-aktive NH-Streckschwingung im cis-cis-Dimer (cc). Außerdem die Verhältnisse der Streuquerschnitte $C$ jeweils für die Monomere $\left(C_{\mathrm{c} / \mathrm{t}}\right)$ und die Dimere $\left(C_{\mathrm{cc} / \mathrm{tt}}\right)$ sowie jeweils deren Mittelwert $(\bar{C})$.

\begin{tabular}{lcccccc}
\hline Spektrum & $\vartheta_{\mathrm{S}}, d$ & $T_{\mathrm{D}} / \mathrm{K}$ & $I(t)$ & $I(c)$ & $F$ & $\Delta G^{\ominus} / \mathrm{kJ} \mathrm{mol}^{-1}$ \\
\hline $130718 \_\mathrm{a}$ & $18^{\circ} \mathrm{C}, 1 \mathrm{~mm}$ & 298 & 10.2 & 0.40 & $0.039(4)$ & $7.6(5)$ \\
$130718 \_\mathrm{b}$ & $18^{\circ} \mathrm{C}, 1 \mathrm{~mm}$ & 323 & 10.5 & 0.41 & $0.039(4)$ & $8.3(6)$ \\
$130718 \_\mathrm{c}$ & $18^{\circ} \mathrm{C}, 1 \mathrm{~mm}$ & 373 & 8.3 & 0.41 & $0.049(5)$ & $8.9(6)$ \\
\hline $130717 \_\mathrm{a}$ & $12^{\circ} \mathrm{C}, 2 \mathrm{~mm}$ & 298 & 2.6 & 0.13 & $0.049(5)$ & $7.1(5)$ \\
$130717 \_\mathrm{b}$ & $12^{\circ} \mathrm{C}, 2 \mathrm{~mm}$ & 353 & 2.4 & 0.13 & $0.056(6)$ & $8.0(6)$ \\
$130717 \_\mathrm{c}$ & $12^{\circ} \mathrm{C}, 2 \mathrm{~mm}$ & 423 & 1.1 & 0.09 & $0.081(8)$ & $8.3(6)$ \\
$130717 \_\mathrm{e}$ & $12^{\circ} \mathrm{C}, 2 \mathrm{~mm}$ & 423 & 2.5 & 0.14 & $0.057(6)$ & $9.5(6)$ \\
\hline $130724 \_\mathrm{a}$ & $5^{\circ} \mathrm{C}, 1 \mathrm{~mm}$ & 298 & 3.6 & 0.19 & $0.052(5)$ & $6.9(5)$ \\
$130724 \_\mathrm{b}$ & $5{ }^{\circ} \mathrm{C}, 1 \mathrm{~mm}$ & 433 & 2.0 & 0.09 & $0.048(5)$ & $10.4(6)$ \\
$130724 \_\mathrm{c}$ & $5{ }^{\circ} \mathrm{C}, 1 \mathrm{~mm}$ & 433 & 2.9 & 0.13 & $0.045(5)$ & $10.6(6)$ \\
\hline $130726 \_\mathrm{a}$ & $5^{\circ} \mathrm{C}, 1 \mathrm{~mm}$ & 298 & 2.3 & 0.12 & $0.056(6)$ & $6.8(5)$ \\
$130726 \_\mathrm{b}$ & $5^{\circ} \mathrm{C}, 1 \mathrm{~mm}$ & 433 & 2.1 & 0.17 & $0.079(8)$ & $8.6(6)$ \\
$130726 \_\mathrm{c}$ & $5^{\circ} \mathrm{C}, 1 \mathrm{~mm}$ & 433 & 2.5 & 0.18 & $0.073(7)$ & $8.9(6)$ \\
\hline $130730 \_\mathrm{b}$ & $5^{\circ} \mathrm{C}, 1 \mathrm{~mm}$ & 298 & 3.3 & 0.16 & $0.049(5)$ & $7.1(5)$ \\
$130730 \_\mathrm{d}$ & $5{ }^{\circ} \mathrm{C}, 1 \mathrm{~mm}$ & 433 & 3.4 & 0.17 & $0.050(5)$ & $10.2(6)$ \\
$130730 \_\mathrm{e}$ & $5{ }^{\circ} \mathrm{C}, 1 \mathrm{~mm}$ & 433 & 3.1 & 0.20 & $0.065(6)$ & $9.3(6)$ \\
\hline
\end{tabular}

Tabelle A.7.4: Relative Intensitäten der NH-Streckschwingungsbande von der trans- $(I(t))$ und cis-NMF $(I(c))$, sowie deren Verhältnis $F$ und die aus den Spektren bestimmte freie Standardenthalpie $\Delta G^{\ominus}(T)$ für verschiedene Düsentemperaturen $T_{\mathrm{D}}$. 


\begin{tabular}{|c|c|c|c|c|c|c|}
\hline \multirow[t]{2}{*}{$T / K$} & \multicolumn{2}{|c|}{$\begin{array}{c}\text { B3LYP/ } \\
6-311++\mathrm{G}(\mathrm{d}, \mathrm{p})\end{array}$} & \multicolumn{2}{|c|}{$\begin{array}{c}\text { B3LYP/ } \\
\text { aVTZ }\end{array}$} & \multicolumn{2}{|c|}{$\begin{array}{l}\text { MP2/ } \\
\text { aVTZ }\end{array}$} \\
\hline & $\begin{array}{c}\Delta H^{\ominus} / \\
\mathrm{kJ} \mathrm{mol}^{-1}\end{array}$ & $\begin{array}{c}\Delta S^{\ominus} / \\
\mathrm{J} \mathrm{mol}\end{array}$ & $\begin{array}{c}\Delta H^{\ominus} / \\
\mathrm{kJ} \mathrm{mol}\end{array}$ & $\begin{array}{c}\Delta S^{\ominus} / \\
\mathrm{J} \mathrm{mol}^{-1} \mathrm{~K}^{-1}\end{array}$ & $\begin{array}{c}\Delta H^{\ominus} / \\
\mathrm{kJ} \mathrm{mol}^{-1}\end{array}$ & $\begin{array}{c}\Delta S^{\ominus} / \\
\mathrm{J} \mathrm{mol}^{-1} \mathrm{~K}^{-1}\end{array}$ \\
\hline 298 & 4.17 & -8.46 & 4.01 & -7.41 & 6.32 & -8.01 \\
\hline 323 & 4.18 & -8.43 & 4.02 & -7.39 & 6.31 & -8.06 \\
\hline 353 & 4.18 & -8.43 & 4.02 & -7.38 & 6.29 & -8.12 \\
\hline 373 & 4.18 & -8.43 & 4.02 & -7.38 & 6.28 & -8.15 \\
\hline 423 & 4.18 & -8.43 & 4.02 & -7.37 & 6.25 & -8.22 \\
\hline 433 & 4.18 & -8.43 & 4.02 & -7.37 & 6.25 & -8.23 \\
\hline
\end{tabular}

Tabelle A.7.5: Auf verschiedenen Niveaus berechnete Standardenthalpien $\left(\Delta H^{\ominus} / \mathrm{kJ}\right.$ $\left.\mathrm{mol}^{-1}\right)$ und -entropien $\left(\Delta S^{\ominus} / \mathrm{J} \mathrm{mol}^{-1} \mathrm{~K}^{-1}\right)$ von NMF für verschiedene Temperaturen. Die Änderung beider Größen mit der Temperatur ist im betrachteten Temperaturbereich vernachlässigbar klein. Der Betrag der Entropie wird im Vergleich zum Experiment von den harmonischen Rechnungen deutlich kleiner vorhergesagt.

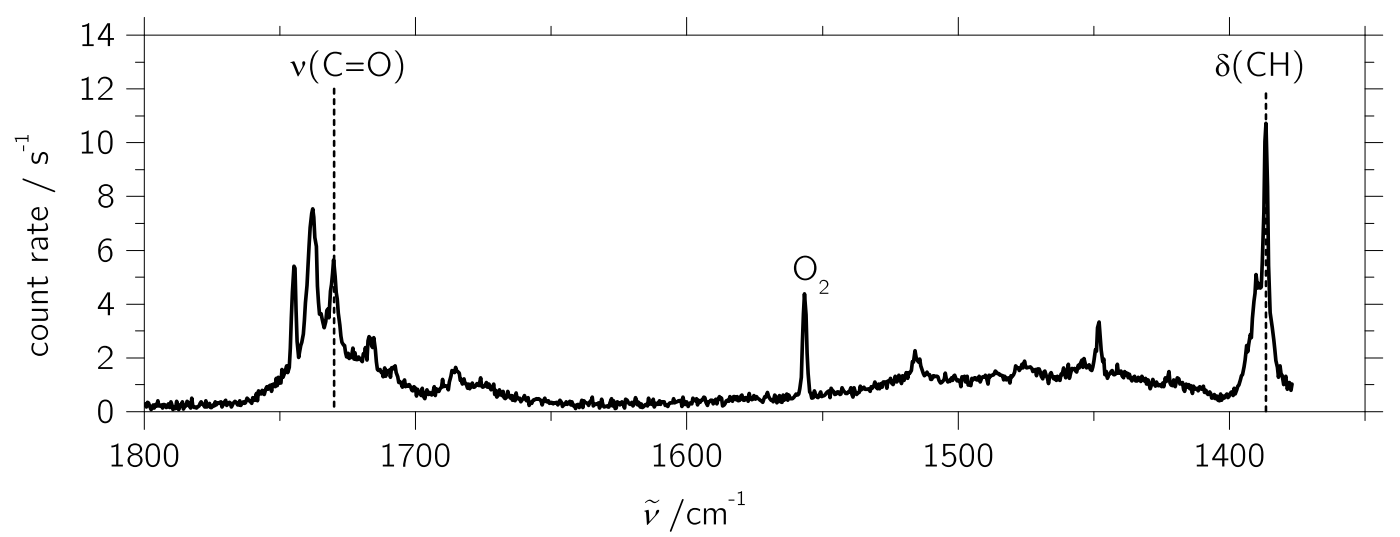

Abbildung A.7.4: Raman-Jetspektrum von NMF im Bereich von $1800-1380 \mathrm{~cm}^{-1}$. Die Carbonylstreckschwingungsbande $(\nu(\mathrm{C}=\mathrm{O}))$ und die $\mathrm{CH}-$ Knickschwingungsbande $(\delta(\mathrm{CH}))$ von trans-NMF, die für eine Kombinationsbande infrage kommen, sind markiert. Außerdem ist eine geringe Verunreinigung mit Luftsauerstoff zu erkennen. Details zu den Messbedingungen finden sich im Anhang A.3. 


\section{A.8 Methanol}

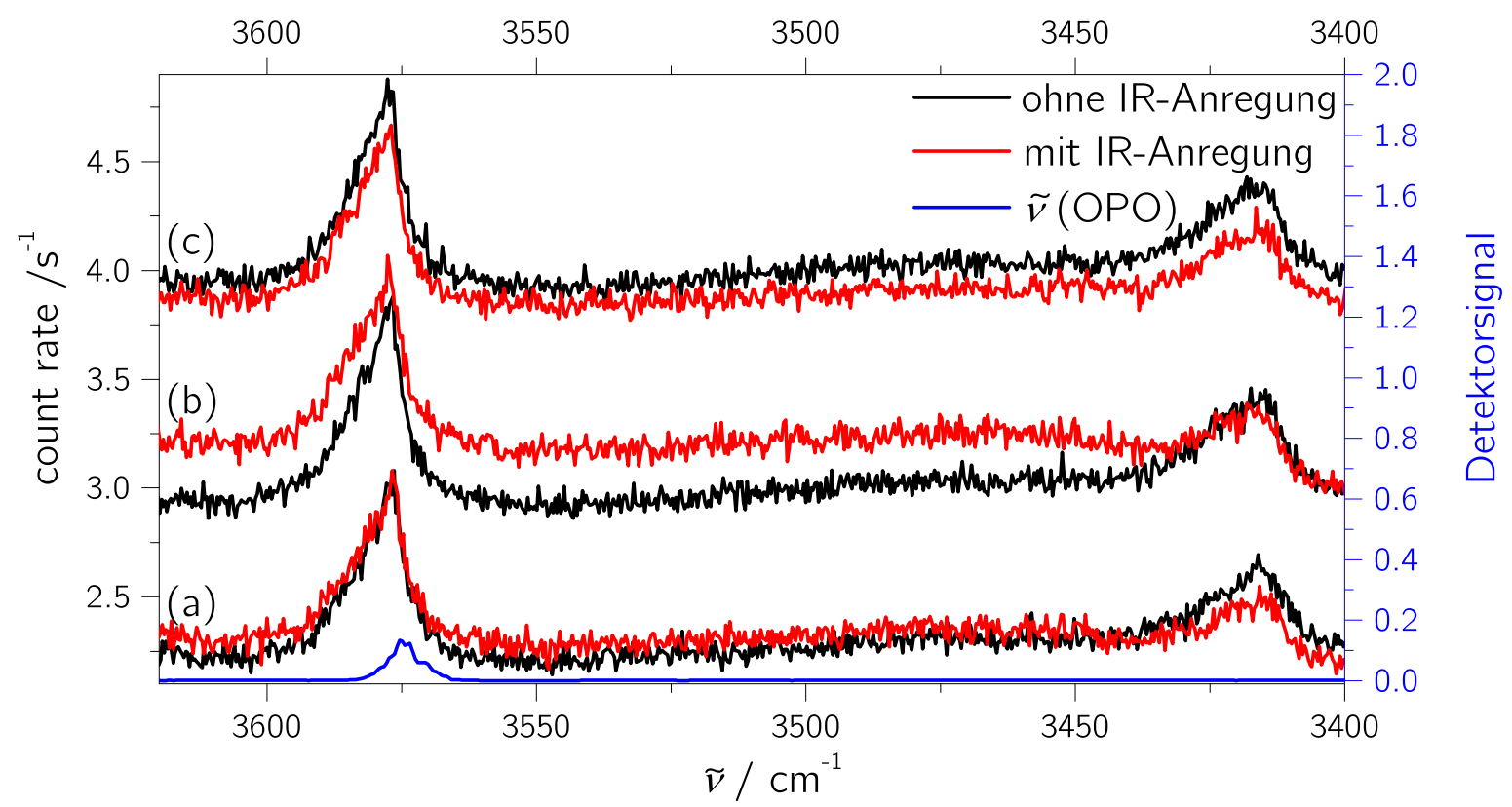

Abbildung A.8.1: Einfluss der Justierung auf die Basislinienänderung bei Nutzung des OPO-Lasers. Bei (a) akzeptabler, (b) schlechter und (c) besonders guter Justierung. In Spektrum (a) ist eine leichte Basislinienerhöhung durch ein schwaches Leuchten der Düse zu erkennen, in (b) führt das Glühen zur Überstrahlung der eigentlichen Signale, die im Extremfall die Auswertung der Spektren unmöglich macht. In Spektrum (c) ist die Basislinie bei IR-Anregung sogar erniedrigt, was wahrscheinlich auf das Aufheizen der Düse, ohne dass diese glüht, zurückzuführen ist, da hierdurch die Bildung größerer insbesondere Ar-umhüllter Spezies, die starke Rayleighstreuer sind, unterdrückt wird. Details zu den Messbedingungen finden sich im Anhang A.3. 


\section{Literaturverzeichnis}

[1] U. Buck, F. Huisken, Infrared Spectroscopy of Size-Selected Water and Methanol Clusters, Chem. Rev. 2000, 100, 3863-3890.

[2] M. Herman, R. Georges, M. Hepp, D. Hurtmans, High Resolution Fourier Transform Spectroscopy of Jet-cooled Molecules, Int. Rev. Phys. Chem. 2000, 19, $277-325$.

[3] T. Häber, U. Schmitt, M. A. Suhm, FTIR-spectroscopy of molecular clusters in pulsed supersonic slit-jet expansions, Phys. Chem. Chem. Phys. 1999, 1, 55735582.

[4] J. B. Paul, R. A. Provencal, C. Chapo, A. Petterson, R. J. Saykally, Infrared cavity ringdown spectroscopy of water clusters: O-D stretching bands, J. Chem. Phys. 1998, 109, 10201-10206.

[5] I. F. Silvera, F. Tommasini, Intracavity Raman Scattering from Molecular Beams: Direct Determination of Local Properties in an Expanding Jet Beam, Phys. Rev. Lett. 1976, 37, 136-140.

[6] H. P. Godfried, I. F. Silvera, Raman studies of argon dimers in a supersonic expansion. I. Spectroscopy, Phys. Rev. A 1983, 27, 3008-3018.

[7] A. Ramos, G. Tejeda, J. M. Fernández, S. Montero, Rotational-translational state-to-state collisional rate constants of $\mathrm{N}_{2}$ at low temperatures $(3<T<16 \mathrm{~K})$, Phys. Rev. A 2002, 66, 022702.

[8] P. Zielke, Ramanstreuung am Überschallstrahl: Wasserstoffbrückendynamik aus neuer Perspektive, Dissertation, Universität Göttingen, 2006.

[9] T. N. Wassermann, Umgebungseinflüsse auf die $C-C$ und $C-O$-Torsionsdynamik in Molekülen und Molekülaggregaten: Schwingungsspektroskopie bei tiefen Temperaturen, Dissertation, Universität Göttingen, 2009.

[10] Z. Xue, Raman spectroscopy of carboxylic acid and water aggregates, Dissertation, Universität Göttingen, 2010.

[11] N. O. B. Lüttschwager, Raman Spectroscopy of Conformational Rearrangements at Low Temperature: Folding and Stretching of Alkanes in Supersonic Jets, Dissertation, Universität Göttingen, 2013. 
[12] R. Wugt Larsen, P. Zielke, M. A. Suhm, Hydrogen-bonded OH stretching modes of methanol clusters: A combined IR and Raman isotopomer study, J. Chem. Phys. 2007, 126, 194307.

[13] P. M. Felker, A. H. Zewail, Ultrafast dynamics of IVR in molecules and reactions, in J. M. Hollas, D. Phillips (Hg.), Jet Spectroscopy and Molecular Dynamics, Kap. 7, Blackie Academic and Professional, Glasgow, UK, 1995, 222-224.

[14] R. M. Balabin, Conformational Equilibrium in Glycine: Experimental Jet-Cooled Raman Spectrum, J. Phys. Chem. Lett. 2010, 1, 20-23.

[15] K. E. Otto, Z. Xue, P. Zielke, M. A. Suhm, The Raman spectrum of isolated water clusters, Phys. Chem. Chem. Phys. 2014, 16, 9849-9858.

[16] T. N. Wassermann, M. A. Suhm, Ethanol Monomers and Dimers Revisited: A Raman Study of Conformational Preferences and Argon Nanocoating, J. Phys. Chem. A 2010, 114, 8223-8233.

[17] M. Heger, Conformational Switching of Weak Hydrogen Bonds: Jet-Cooled Torsional States of Short-Chain Molecules, Masterarbeit, Universität Göttingen, 2012.

[18] M. Heger, K. E. Otto, R. A. Mata, M. A. Suhm, Bracketing subtle conformational energy differences between self-solvated and stretched trifluoropropanol, Phys. Chem. Chem. Phys. 2015, 17, 9899-9909.

[19] J. B. Paul, R. A. Provencal, R. J. Saykally, Characterization of the $\left(\mathrm{D}_{2} \mathrm{O}\right)_{2}$ Hydrogen-Bond-Acceptor Antisymmetric Stretch by IR Cavity Ringdown Laser Absorption Spectroscopy, J. Phys. Chem. A 1998, 102, 3279-3283.

[20] L. M. Goss, S. W. Sharpe, T. A. Blake, V. Vaida, J. W. Brault, Direct Absorption Spectroscopy of Water Clusters, J. Chem. Phys. A 1999, 103, 8620-8624.

[21] D. J. Nesbitt, T. Häber, M. A. Suhm, General Discussion, Faraday Discuss. 2001, 118, 304-309.

[22] A. Moudens, R. Georges, M. Goubet, J. Makarewicz, S. E. Lokshtanov, A. A. Vigasin, Direct absorption spectroscopy of water clusters formed in a continuous slit nozzle expansion, J. Chem. Phys. 2009, 131, 204312.

[23] F. J. Lovas, S. P. Belov, M. Y. Tretyakov, W. Stahl, R. D. Suenram, The a-Type $K=0$ Microwave Spectrum of the Methanol Dimer, J. Mol. Spectrosc. 1995, 170, 478-492.

[24] R. A. Provencal, J. B. Paul, K. Roth, C. Chapo, N. Casaes, R. J. Saykally, G. S. Tschumper, H. F. Schaefer III, Infrared cavity ringdown spectroscopy of methanol clusters: Single donor hydrogen bonding, J. Chem. Phys. 1999, 110, 4258-4267. 
[25] S. Coussan, Y. Bouteiller, A. Loutellier, J. P. Perchard, S. Racine, A. Peremans, W. Q. Zheng, A. Tadjeddine, Infrared photoisomerization of the methanol dimer trapped in argon matrix: monochromatic irradiation experiments and DFT calculations, Chem. Phys. 1997, 219, 221-234.

[26] D. A. Long, The Raman Effect - A Unified Treatment of the Theory of Raman Scattering by Molecules, John Wiley \& Sons, Chichester UK, 2002.

[27] M. D. Morse, Supersonic Beam Sources, in F. Dunning, R. G. Hulet (Hg.), Atomic, Molecular, and Optical Physics: Atoms and Molecules, Bd. 29, Part B von Experimental Methods in the Physical Sciences, Academic Press, 1996, 21 - 47.

[28] J. M. Hayes, Analytical Spectroscopy in Supersonic Expansions, Chem. Rev. 1987, 87, 745-760.

[29] R. E. Smalley, L. Wharton, D. H. Levy, Molecular Optical Spectroscopy with Supersonic Beams and Jets, Acc. Chem. Res. 1977, 10, 139-145.

[30] O. F. Hagena, Nucleation and growth of clusters in expanding nozzle flows, Surf. Sci. 1981, 106, 101-116.

[31] R. S. Ruoff, T. D. Klots, T. Emilsson, H. S. Gutowsky, Relaxation of Conformers and Isomers in Seeded Supersonic Jets of Inert Gases, J. Chem. Phys. 1990, 93, $3142-3150$.

[32] C. Manneback, Die Intensität und Polarisation der von zweiatomigen Molekülen gestreuten kohärenten und inkohärenten Strahlung, Z. Phys. A 1930, 62, 224252.

[33] C. Manneback, Berichtigung zu meiner Arbeit "Die Intensität und Polarisation der von zweiatomigen Molekülen gestreuten kohärenten und inkohärenten Strahlung", Z. Phys. A 1930, 65, 574.

[34] M. J. Frisch, G. W. Trucks, H. B. Schlegel, G. E. Scuseria, M. A. Robb, J. R. Cheeseman, G. Scalmani, V. Barone, B. Mennucci, G. A. Petersson, H. Nakatsuji, M. Caricato, X. Li, H. P. Hratchian, A. F. Izmaylov, J. Bloino, G. Zheng, J. L. Sonnenberg, M. Hada, M. Ehara, K. Toyota, R. Fukuda, J. Hasegawa, M. Ishida, T. Nakajima, Y. Honda, O. Kitao, H. Nakai, T. Vreven, J. J. A. Montgomery, J. E. Peralta, F. Ogliaro, M. Bearpark, J. J. Heyd, E. Brothers, K. N. Kudin, V. N. Staroverov, R. Kobayashi, J. Normand, K. Raghavachari, A. Rendell, J. C. Burant, S. S. Iyengar, J. Tomas, M. Cossi, N. Rega, J. M. Millam, M. Klene, J. E. Knox, J. B. Cross, V. Bakken, C. Adamo, J. Jaramillo, R. Gomperts, R. E. Stratmann, O. Yazyev, A. J. Austin, R. Cammi, C. Pomelli, J. W. Ochterski, R. L. Martin, K. Morokuma, V. G. Zakrzewski, G. A. Voth, P. Salvador, J. J. Dannenberg, S. Dapprich, A. D. Daniels, O. Farkas, J. B. Foresman, J. V. Ortiz, J. Cioslowski, D. J. Fox, Gaussian 09, Revision A.02, 2009. 
[35] TURBOMOLE V6.5 2013, a development of University of Karlsruhe and Forschungszentrum Karlsruhe GmbH, 1989-2007, TURBOMOLE GmbH, since 2007; available from

http://www. turbomole.com.

[36] P. W. Atkins, Physikalische Chemie, 3. Aufl., Wiley-VCH, Weinheim, 2004.

[37] G. Wedler, Lehrbuch der Physikalischen Chemie, 5. Aufl., Wiley-VCH, Weinheim, 2004.

[38] S. Graf, W. Mohr, S. Leutwyler, An ab initio study of the torsional-puckering pseudorotation in the cyclic water petamer, J. Chem. Phys. 1999, 110, 78937908 .

[39] W. Demtröder, Laserspektroskopie, 5. Aufl., Springer, Berlin, 2007.

[40] G. A. Reider, Photonik - Eine Einführung in die Grundlagen, Springer, Wien, 2012.

[41] J. Eichler, H. J. Eichler, Laser - Bauformen, Strahlführung, Anwendungen, Springer, Berlin, 2010.

[42] H. J. Eichler, A. Fleischer, J. Kross, M. Krystek, H. Lang, H. Niedrig, H. Rauch, G. Schmahl, H. Schoenebeck, E. Sedlmayr, H. Weber, K. Weber, Lehrbuch der Experimentalphysik, Band 3 Optik, 9. Aufl., Walter de Gruyter, Berlin, 1993.

[43] Lockheed Martin Aculight, 22121 -20th Avenue SE Bothell, WA 98021, Argos Model 2400 CW OPO User Manual.

[44] D. Meschede, Optics, Light and Lasers, 2. Aufl., Wiley-VCH, Weinheim, 2007.

[45] D. S. Hum, M. M. Fejer, Recent advances in crystal optics: Quasi-phasematching, C. R. Physique 2007, 8, 180-198.

[46] L. E. Myers, R. C. Eckardt, M. M. Fejer, R. L. Byer, W. R. Rosenberg, J. W. Pierce, Quasi-phase-matched optical parametric oscillators in bulk periodically poled $\mathrm{LiNbO}_{3}$, J. Opt. Soc. Am. B 1995, 12, 2102-2116.

[47] R. Hildebrandt, V. Meyer, Technische Zeichnung: Montage Vakuumkammer, Zeichenbüro, Institut für Physikalische Chemie der Universität Göttingen, 2013.

[48] V. Meyer, Persönliche Mitteilung, 2014.

[49] K.-H. Bernhardt, (Pfeiffer Vacuum GmbH), Vacuum Technology Compendium, 2010.

[50] Leybold AG, Grundlagen der Vakuumtechnik, Berechnungen und Tabellen, 1987. 
[51] A. M. Morrison, J. Agarwal, H. F. Schaefer III, G. E. Douberly, Infrared Laser Spectroscopy of the $\mathrm{CH}_{3} \mathrm{OO}$ Radical Formed from the Reaction of $\mathrm{CH}_{3}$ and $\mathrm{O}_{2}$ within a Helium Nanodroplet, J. Phys. Chem. A 2012, 116, 5299-5304.

[52] A. M. Morrison, T. Liang, G. E. Douberly, Automation of an "Aculight"continuous-wave optical parametric oscillator, Rev. Sci. Instrum. 2013, 84, 03102.

[53] C. C. Pradzynski, R. M. Forck, T. Zeuch, P. Slavícek, U. Buck, A Fully SizeResolved Perspective on the Crystallization of Water Clusters, Science 2012, 337, 1529-1532.

[54] D. J. Nesbitt, R. W. Field, Vibrational Energy Flow in Highly Excited Molecules: Role of Intramolecular Vibrational Redistribution, J. Phys. Chem. 1996, 100, $12735-12756$.

[55] K. K. Lehmann, G. Scoles, B. H. Pate, Intramolecular Dynamics from EigenstateResolved Infrared Spectra, Annu. Rev. Phys. Chem. 1994, 45, 241-74.

[56] R. E. Miller, Infrared Laser Photodissociation and Spectroscopy of van der Waals Molecules, J. Phys. Chem. 1986, 90, 3301-3313.

[57] J. Zischang, Strukturdynamik wasserhaltiger Aggregate in Überschallexpansionen, Dissertation, Universität Göttingen, 2014.

[58] J. Zischang, M. A. Suhm, The OH stretching spectrum of warm water clusters, J. Chem. Phys. 2014, 140, 064312.

[59] T. Kalkowski, Aggregation von fluorierten Alkoholen, Diplomarbeit, Universität Göttingen, 2004.

[60] T. Scharge, Schwache Bindungen mit starker Wirkung: Konformation und Aggregation von halogenierten Alkoholen, Dissertation, Universität Göttingen, 2007.

[61] NKT Photonics GmbH, Quotation Quote No Q200080-1 Argos Model 2400-BB30 (B), 2012.

[62] A. Henderson, Persönliche Mitteilung, 2012.

[63] P. R. Griffiths, J. A. de Haseth, Fourier Transform Infrared Spectrometry, 2. Aufl., John Wiley \& Sons, Hoboken, 2007.

[64] http://www.spectralcalc.com/blackbody_calculator/blackbody.php, Stand 18. August 2014.

[65] B. Maté, G. Tejeda, S. Montero, Raman spectroscopy of supersonic jets of $\mathrm{CO}_{2}$ : Density, condensation, and translational, rotational, and vibrational temperatures, J. Chem. Phys. 1998, 108, 2676-2685. 
[66] A. Ramos, B. Maté, G. Tejeda, J. M. Fernández, S. Montero, Raman spectroscopy of hypersonic shock waves, Phys. Rev. E 2000, 62, 4940-4945.

[67] S. Hesse, T. N. Wassermann, M. A. Suhm, Brightening and Locking a Weak and Floppy N-H Chromophor: The Case of Pyrrolidine, J. Phys. Chem. A 2010, 114, 10492-10499.

[68] G. Tejeda, F. Thibault, J. M. Fernández, S. Montero, Low-temperature inelastic collisions between hydrogen molecules and helium atoms, J. Chem. Phys. 2008, 128, 224308.

[69] B. J. Kip, R. J. Meier, Determination of the Local Temperature at a Sample During Raman Experiments Using Stokes and Anti-Stokes Raman Bands, Appl. Spectrosc. 1990, 44, 707-711.

[70] A. T. Hartlieb, B. Atakan, K. Kohse-Höinghaus, Temperature measurement in fuel-rich non-sooting low-pressure hydrocarbon flames, Appl. Phys. B 2000, 70, 435-445.

[71] S. D. Rassat, E. J. Davis, Temperature Measurement of Single Levitated Microparticles Using Stokes/Anti-Stokes Raman Intensity Ratios, Appl. Spectrosc. 1994, 48, 1498-1505.

[72] S. P. Nikitin, C. Manka, J. Grun, J. Bowles, A technique for contactless measurement of water temperature using Stokes and anti-Stokes comparative Raman spectroscopy, Rev. Sci. Instrum. 2012, 83, 033105.

[73] H. Tsuji, A. Oda, J. Kido, T. Sugiyama, Y. Furukawa, Temperature Measurements of Organic Light-Emitting Diodes by Stokes and Anti-Stokes Raman Scattering, Jap. J. Appl. Phys. 2008, 47, 2171.

[74] J. Bendtsen, The Rotational and Rotational-Vibrational Raman Spectra of ${ }^{14} \mathrm{~N}_{2}$, ${ }^{14} \mathrm{~N}^{15} \mathrm{~N}$ and ${ }^{15} \mathrm{~N}_{2}$, J. Raman Spectrosc. 1974, 2, 133-145.

[75] F. LaPlant, G. Laurence, D. Ben-Amotz, Theoretical and Experimental Uncertainty in Temperature Measurement of Materials by Raman Spectroscopy, Appl. Spectrosc. 1996, 50, 1034-1038.

[76] P. H. Krupenie, The Spectrum of Molecular Oxygen, J. Phys. Chem. Ref. Data 1972, 1, 425-534.

[77] G. Avila, G. Tejeda, J. M. Fernández, S. Montero, The rotational Raman spectra and cross sections of $\mathrm{H}_{2} \mathrm{O}, \mathrm{D}_{2} \mathrm{O}$ and HDO, J. Mol. Spectrosc. 2003, 220, 259-275.

[78] G. Tejeda, M. Fernández, S. Montero, Diagnostics of $\mathrm{H}_{2} \mathrm{O}$ and $\mathrm{H}_{2} \mathrm{O}+\mathrm{He}$ Supersonic Jets by Raman Spectroscopy, AIP Conf. Proc. 2012, 1501, 1305. 
[79] G. Avila, J. M. Fernández, G. Tejeda, S. Montero, The Raman spectra and crosssections of $\mathrm{H}_{2} \mathrm{O}, \mathrm{D}_{2} \mathrm{O}$ and $\mathrm{HDO}$ in the $\mathrm{OH} / \mathrm{OD}$ stretching regions, J. Mol. Spectrosc. 2004, 228, 38-65.

[80] F. Kollipost, Schwingungsdynamik in O-H*O-verbrückten Aggregaten: FTIRSpektroskopie vom Nah- bis zum Ferninfraroten, Dissertation, Universität Göttingen, 2015.

[81] R. Ludwig, Wasser: von Clustern in die Flüssigkeit, Angew. Chem. 2001, 113, $1856-1876$.

[82] H. J. Bakker, J. L. Skinner, Vibrational Spectroscopy as a Probe of Structure and Dynamics in Liquid Water, Chem. Rev. 2010, 110, 1498-1517.

[83] S. Woutersen, H. J. Bakker, Resonant intermolecular transfer of vibrational energy in liquid water, Nature 1999, 402, 507-509.

[84] M. L. Cowan, B. D. Bruner, N. Huse, J. R. Dwyer, B. Chugh, E. T. J. Nibbering, T. Elsaesser, R. J. D. Miller, Ultrafast memory loss and energy redistribution in the hydrogen bond network of liquid $\mathrm{H}_{2} \mathrm{O}$, Nature 2005, 434, 199-202.

[85] H. Torii, Time-Domain Calculations of the Polarized Raman Spectra, the Transient Infrared Absorption Anisotropy, and the Extent of Delocalization of the $\mathrm{OH}$ Stretching Mode of Liquid Water, J. Phys. Chem. A 2006, 110, 9469-9477.

[86] A. Paarmann, T. Hayashi, S. Mukamel, R. J. D. Miller, Probing intermolecular couplings in liquid water with two-dimensional infrared photon echo spectroscopy, J. Chem. Phys. 2008, 128, 191103.

[87] F. N. Keutsch, R. J. Saykally, Water clusters: Untangling the mysteries of the liquid, one molecule at a time, Proc. Natl. Acad. Sci. 2001, 98, 10533-10540.

[88] G. E. Walrafen, Raman Spectral Studies of Water Structure, J. Chem. Phys. 1964, 40, 3249-3256.

[89] J. Ceponkus, P. Uvdal, B. Nelander, On the structure of the matrix isolated water trimer, J. Chem. Phys. 2011, 134, 064309.

[90] B. Tremblay, B. Madebène, M. E. Alikhani, J. P. Perchard, The vibrational spectrum of the water trimer: Comparison between anharmonic ab initio calculations and neon matrix infrared data between 11,000 and $90 \mathrm{~cm}^{-1}$, Chem. Phys. 2010, $378,27-36$.

[91] S. Y. Fredericks, J. M. Pedulla, K. D. Jordan, T. S. Zwier, OH stretch IR spectra of $\left(\mathrm{H}_{2} \mathrm{O}\right)_{3}$ and benzene- $\left(\mathrm{H}_{2} \mathrm{O}\right)_{3}$, Theor. Chem. Acc. 1997, 96, 51-55. 
[92] G. C. Groenenboom, E. M. Mas, R. Bukowski, K. Szalewicz, P. E. S. Wormer, A. van der Avoird, Water Pair and Three-Body Potential of Spectroscopic Quality from Ab Initio Calculations, Phys. Rev. Lett. 2000, 84, 4072-4075.

[93] C. Pérez, M. T. Muckle, D. P. Zaleski, N. A. Seifert, B. Temelso, G. C. Shields, Z. Kisiel, B. H. Pate, Structures of Cage, Prism, and Book Isomers of Water Hexamer from Broadband Rotational Spectroscopy, Science 2012, 336, 897-901.

[94] S. Wuelfert, D. Herren, S. Leutwyler, Supersonic jet CARS spectra of small water clusters, J. Chem. Phys. 1987, 86, 3751-3753.

[95] B. Nelander, The intramolecular fundamentals of the water dimer, J. Chem. Phys. 1988, 88, 5254-5256.

[96] S. Wuelfert, D. Herren, S. Leutwyler, Reply to "The intramolecular fundamentals of the water dimer", J. Chem. Phys. 1988, 88, 5256-5257.

[97] G. Firanescu, D. Luckhaus, R. Signorell, Phase, shape, and architecture of $\mathrm{SF}_{6}$ and $\mathrm{SF}_{6} / \mathrm{CO}_{2}$ aerosol particles: Infrared spectra and modeling of vibrational excitons, J. Chem. Phys. 2008, 128, 184301.

[98] Y. Wang, V. Babin, J. M. Bowman, F. Paesani, The Water Hexamer: Cage, Prism, or Both. Full Dimensional Quantum Simulations Say Both, J. Am. Chem. Soc. 2012, 134, 11116-11119.

[99] K. Kuyanov-Prozument, M. Y. Choi, A. F. Vilesov, Spectrum and infrared intensities of OH-stretching bands of water dimers, J. Chem. Phys. 2010, 132, 014304 .

[100] S. S. Xantheas, T. H. Dunning, Ab initio studies of cyclic water clusters $\left(\mathrm{H}_{2} \mathrm{O}\right)_{n}$, $\mathrm{n}=1-6$. Optimal structures and vibrational spectra, J. Chem. Phys. 1993, 99, $8774-8792$.

[101] J. Kim, K. S. Kim, Structures, binding energies, and spectra of isoenergetic water hexamer clusters: Extensive ab initio studies, J. Chem. Phys. 1998, 109, 58865895 .

[102] M. A. Suhm, How Broad Are Water Dimer Bands? (Letter to the Editor), Science 2004, 304, 823 .

[103] M. Y. Tretyakov, E. A. Serov, M. A. Koshelev, V. V. Parshin, A. F. Krupnov, Water Dimer Rotationally Resolved Millimeter-Wave Spectrum Observation at Room Temperature, Phys. Rev. Lett. 2013, 110, 093001.

[104] Y. Wang, B. C. Shepler, B. J. Braams, J. M. Bowman, Full-dimensional, ab initio potential energy and dipole moment surfaces for water, J. Chem. Phys. 2009, 131, 054511. 
[105] H. Cybulski, J. Sadlej, On the calculations of the vibrational Raman spectra of small water clusters, Chem. Phys. 2007, 342, 163-172.

[106] Y. Watanabe, S. Maeda, K. Ohno, Intramolecular vibrational frequencies of water clusters $\left(\mathrm{H}_{2} \mathrm{O}\right)_{n}(\mathrm{n}=2-5)$ : Anharmonic analyses using potential functions based on the scaled hypersphere search method, J. Chem. Phys. 2008, 129, 074315.

[107] Y. Wang, J. M. Bowman, Coupled-monomers in molecular assemblies: Theory and application to the water tetramer, pentamer, and ring hexamer, J. Chem. Phys. 2012, 136, 144113.

[108] E. Miliordos, E. Aprà, S. S. Xantheas, Optimal geometries and harmonic vibrational frequencies of the global minima of water clusters $\left(\mathrm{H}_{2} \mathrm{O}\right)_{n}, \mathrm{n}=2-6$, and several hexamer local minima at the CCSD(T) level of theory, J. Chem. Phys. 2013, 139, 114302.

[109] Y. Wang, X. Huang, B. C. Shepler, B. J. Braams, J. M. Bowman, Flexible, ab initio potential, and dipole moment surface for water. I. Tests and applications for clusters up to the 22-mer, J. Chem. Phys. 2011, 134, 094509.

[110] C. J. Burnham, S. S. Xantheas, M. A. Miller, B. E. Applegate, R. E. Miller, The formation of cyclic water complexes by sequential ring insertion: Experiment and theory, J. Chem. Phys. 2002, 117, 1109-1122.

[111] J. Ceponkus, G. Karlström, B. Nelander, Intermolecular Vibrations of the Water Trimer, a Matrix Isolation Study, J. Phys. Chem. A 2005, 109, 7859-7864.

[112] J. Ceponkus, P. Uvdal, B. Nelander, Water Tetramer, Pentamer, and Hexamer in Inert Matrices, J. Phys. Chem. A 2012, 116, 4842-4850.

[113] Y. Wang, J. M. Bowman, Flexible, ab initio potential, and dipole moment surface for water. II. Local-monomer calculations of the infrared spectra of water clusters, J. Chem. Phys. 2011, 134, 154510.

[114] H. Q. Harker, M. R. Viant, F. N. Keutsch, E. A. Michael, R. P. McLaughlin, R. J. Saykally, Water Pentamer: Characterization of the Torsional-Puckering Manifold by Terahertz VRT Spectroscopy, J. Phys.Chem. A 2005, 109, 6483-6497.

[115] C. Steinbach, P. Andersson, M. Melzer, J. K. Kazimirski, U. Buck, V. Buch, Detection of the book isomer from the OH-stretch spectroscopy of size selected water hexamers, Phys. Chem. Chem. Phys. 2004, 6, 3320-3324.

[116] J. B. Paul, R. A. Provencal, C. Chapo, K. Roth, R. Casaes, R. J. Saykally, Infrared Cavity Ringdown Spectroscopy of the Water Cluster Bending Vibrations, J. Phys. Chem. A 1999, 103, 2972-2974.

\section{XXXVIII}


[117] S. J. Daunt, H. F. Shurvell, The Gas Phase Infrared Band Contour of $s$-Triazine and $s$-Triazine- $d_{3}$ The Fundamental of $\mathrm{C}_{3} \mathrm{~N}_{3} \mathrm{H}_{3}$ and $\mathrm{C}_{3} \mathrm{~N}_{3} \mathrm{D}_{3}$ and Some Overtone and Combination Bands of $\mathrm{C}_{3} \mathrm{~N}_{3} \mathrm{H}_{3}$, J. Mol. Spectrosc. 1976, 62, 373-395.

[118] Y. Wang, J. M. Bowman, IR Spectra of the Water Hexamer: Theory, with Inclusion of the Monomer and Overtone, and Experiment Are in Agreement, J. Phys. Chem. Lett. 2013, 4, 1104-08.

[119] E. G. Diken, W. H. Robertson, M. A. Johnson, The Vibrational Spectrum of the Neutral $\left(\mathrm{H}_{2} \mathrm{O}\right)_{6}$ Precursor to the "Magic" $\left(\mathrm{H}_{2} \mathrm{O}\right)_{6}^{-}$Cluster Anion by ArgonMediated, Population-Modulated Electron Attachment Spectroscopy, J. Phys. Chem. A 2004, 108, 64-68.

[120] D. Forney, M. E. Jacox, W. E. Thompson, The Mid- and Near-Infrared Spectra of Water and Water Dimer Isolated in Solid Neon, J. Mol. Spectrosc. 1993, 15\%, 479-493.

[121] G. Avila, G. Tejeda, J. M. Fernández, S. Montero, The Raman spectra and crosssections of the $\nu_{2}$ band of $\mathrm{H}_{2} \mathrm{O}, \mathrm{D}_{2} \mathrm{O}$, and HDO, J. Mol. Spectrosc. 2004, 223, $166-180$.

[122] R. M. Bentwood, A. J. Barnes, W. J. Orville-Thomas, Studies of Intermolecular Interactions by Matrix Isolation Vibrational Spectroscopy, J. Mol. Spectrosc. 1980, 84, 391-404.

[123] Y. Bouteiller, J. P. Perchard, The vibrational spectrum of $\left(\mathrm{H}_{2} \mathrm{O}\right)_{2}$ : comparison between anharmonic ab initio calculations and neon matrix infrared data between 9000 and $90 \mathrm{~cm}^{-1}$, Chem. Phys. 2004, 305, 1-12.

[124] S. A. Nizkorodov, M. Ziemkiewicz, D. J. Nesbitt, A. E. W. Knight, Overtone spectroscopy of $\mathrm{H}_{2} \mathrm{O}$ clusters in the $v_{\mathrm{OH}}=2$ manifold: Infrared-ultraviolet vibrationally mediated dissociation studies, J. Chem. Phys. 2005, 122, 194316.

[125] T. Földes, T. Vanfleteren, M. Herman, Communication: A rotationally resolved $(2 \mathrm{OH})$ overtone band in the water dimer $\left(\mathrm{H}_{2} \mathrm{O}\right)_{2}, J$. Chem. Phys. 2014, 141, 111103 .

[126] T. Scharge, D. Luckhaus, M. A. Suhm, Observation and quantification of the hydrogen bond effect on $\mathrm{O}-\mathrm{H}$ overtone intensities in an alcohol dimer, Chem. Phys. 2008, 346, 267-275.

[127] F. Kollipost, K. Papendorf, Y.-F. Lee, Y.-P. Lee, M. A. Suhm, Alcohol dimers - how much diagonal OH anharmonicity?, Phys. Chem. Chem. Phys. 2014, 16, 15948. 
[128] H. G. Kjaergaard, A. L. Garden, G. M. Chaban, R. B. Gerber, D. A. Matthews, J. F. Stanton, Calculation of Vibrational Transition Frequencies and Intensities in Water Dimer: Comparison of Different Vibrational Approaches, J. Chem. Phys. A 2008, 112, 4324-4335.

[129] M. A. Suhm, Hydrogen Bond Dynamics in Alcohol Clusters, in S. A. Rice (Hg.), Advances in Chemical Physics, Volume 142, Bd. 142 von Advances in Chemical Physics, Kap. 1, Wiley-VCH, 2009, 1-57.

[130] M. Fárník, D. J. Nesbitt, Intramolecular energy transfer between oriented chromophores: High-resolution infrared spectroscopy of $\mathrm{HCl}$ trimer, J. Chem. Phys. 2004, 121, 12386-12395.

[131] C. J. Tainter, J. L. Skinner, The water hexamer: Three-body interactions, structures, energetics, and $\mathrm{OH}$-stretch spectroscopy at finite temperature, J. Chem. Phys. 2012, 137, 104304.

[132] J. Lindner, P. Vöhringer, M. S. Pshenichnikov, D. Cringus, D. A. Wiersma, M. Mostovoy, Vibrational relaxation of pure liquid water, Chem. Phys. Lett. 2006, 421, $329-333$.

[133] Y. L. Niu, R. Pang, C. Y. Zhu, M. Hayashi, Y. Fujimura, S. H. Lin, Y. R. Shen, Quantum chemical calculation of intramolecular vibrational redistribution and vibrational energy transfer of water clusters, Chem. Phys. Lett. 2013, 586, 153 158.

[134] M. Perttilä, Vibrational spectra and normal coordinate analysis of 2,2,2trichloroethanol and 2,2,2-trifluoroethanol, Spectrochim. Acta Part A 1979, 35, 585-592.

[135] V. F. Kalasinsky, H. V. Anjarla, Vibrational Spectra and Conformations of 2,2,2Trifluoroethylamine and 2,2,2-Trifluoroethanol, J. Phys. Chem. 1980, 84, 19401944.

[136] J. R. Durig, R. A. Larsen, Torsional Vibrations and Barriers to Internal Rotation for Ethanol and 2,2,2-Trifluoroethanol, J. Mol. Struct. 1989, 238, 195-222.

[137] J. Marco, J. M. Orza, Vapor phase FTIR study of H-bonded complexes of 2,2,2Trifluoroethanol and its deuterated derivatives, J. Mol. Struct. 1992, 267, 33-38.

[138] L.-H. Xu, G. T. Fraser, F. J. Lovas, R. D. Suenram, C. W. Gillies, H. E. Warner, J. Z. Gillies, The microwave spectrum and $\mathrm{OH}$ internal rotation dynamics of gauche-2,2,2-trifluoroethanol, J. Chem. Phys. 1995, 103, 9541-9548.

[139] I. Bakó, T. Radnai, M.-C. Bellisent-Funel, Investigation of structure of liquid 2,2,2-trifluoroethanol: Neutron diffraction, molecular dynamics, and ab initio quantum chemical study, J. Chem. Phys. 2004, 121, 12472-12480. 
[140] T. Scharge, T. Häber, M. A. Suhm, Quantitative chirality synchronization in trifluoroethanol dimers, Phys. Chem. Chem. Phys. 2006, 8, 4664-4667.

[141] T. Scharge, C. Cézard, P. Zielke, A. Schütz, C. Emmeluth, M. A. Suhm, A peptide co-solvent under scrutiny: self-aggregation of 2,2,2-triflouroethanol, Phys. Chem. Chem. Phys. 2007, 9, 4472-4490.

[142] K.-M. Marstokk, H. Møllendal, Microwave Spectrum, Intramolecular Hydrogen Bonding, Conformational Properties and Quantum Chemical Calculations for 3,3,3-Trifluoropropanol, Acta Chem. Scand. 1999, 53, 202-208.

[143] P. Felder, H. H. Günthard, Conformational interconversions in supersonic jets: Matrix IR spectroscopy and model calculations, Chem. Phys. 1982, 71, 9-25.

[144] N. O. B. Lüttschwager, M. A. Suhm, Stretching and folding of 2-nanometer rods, Soft Matter 2014, 10, 4885-4901.

[145] M. Albrecht, C. A. Rice, M. A. Suhm, Elementary Peptide Motifs in the Gas Phase: FTIR Aggregation Study of Formamide, Acetamide, $N$-Methylformamide and N-Methylacetamide, J. Phys. Chem. A 2008, 112, 7530-7542.

[146] M. Albrecht, Biologisch relevante Wasserstoffbrückensysteme im Überschallstrahl: Steuerung der Aggregation durch Substitution, Dissertation, Universität Göttingen, 2009.

[147] J. J. Lee, Adaptive Aggregation über starke Wasserstoffbrücken in der Gasphase, Dissertation, Universität Göttingen, 2012.

[148] A. C. Fantoni, W. Caminati, Rotational spectrum and ab initio calculations of $N$-methylformamide, J. Chem. Soc. Faraday Trans. 1996, 92, 343-346.

[149] S. Ataka, H. Takeuchi, M. Tasumi, Infrared studies of the less stable cis form of $N$-methylformamide and $N$-methylacetamide in low-temperature nitrogen matrices and vibrational analyses of the trans and cis forms of these molecules, $J$. Mol. Struct. 1984, 113, 147-160.

[150] S. Shin, A. Kurawaki, Y. Hamada, K. Shinya, K. Ohno, A. Tohara, M. Sato, Conformational behavior of $N$-methylformamide in the gas, matrix and solution states as revealed by IR and NMR spectroscopic measurements and by theoretical calculations, J. Mol. Struct. 2006, 791, 30-40.

[151] Y. Sugawara, A. Y. Hirakawa, M. Tsuboi, S. Kato, K. Morokuma, Force constants of trans and cis $N$-methylformamide from ab initio SCF MO Calculations, Chem. Phys. 1981, 62, 339-351. 
[152] A. García Martínez, E. Teso Vilar, A. García Fraile, P. Martínez-Ruiz, A Computational and Experimental Study on the Relative Stabilities of the Cis and Trans Conformers of $N$-Alkylamides in Gas Phase and in Solution, J. Phys. Chem. A 2002, 106, 4942-4950.

[153] R. Crespo-Otero, A. Mardyukov, E. Sanchez-Garcia, M. Barbatti, W. Sander, Photochemistry of $N$-Methylformamide: Matrix Isolation and Nonadiabatic Dynamics, Chem. Phys. Chem. 2013, 14, 827-836.

[154] A. F. Fantoni, W. Caminati, H. Hartwig, W. Stahl, The very low methyl group $V_{3}$ barrier of cis $N$-methylformamide: A-E doubling from the free jet rotational spectrum, J. Mol. Struct. 2002, 6, 305-307.

[155] G. Nandini, D. N. Sathyanarayana, Ab initio studies on geometry and vibrational spectra of $N$-Methylformamide and $N$-Methylacetamide, J. Mol. Struct. (Theochem) 2002, 579, 1-9.

[156] T. L. Teuteberg, Das Dimer des Acetons, Bachlorarbeit, Universität Göttingen, 2011.

[157] M. Sałdyka, Z. Mielke, K. Mierzwicki, S. Coussan, P. Roubin, CH stretching vibration of $N$-Methylformamide as a sensitive probe of its complexation: infrared matrix isolation and computational study, Phys. Chem. Chem. Phys. 2011, 13, 13992-14002.

[158] R. H. Hunt, W. N. Shelton, F. A. Flaherty, W. B. Cook, Torsion-Rotation Energy Levels and the Hindering Potential Barrier for the Excited Vibrational State of the OH-Stretch Fundamental Band $\nu_{1}$ of Methanol, J. Mol. Spectrosc. 1998, 192, $277-293$.

[159] F. Huisken, A. Kulcke, C. Laush, J. M. Lisy, Dissociation of small methanol clusters after excitation of the $\mathrm{O}-\mathrm{H}$ stretch vibration at $2.7 \mu, \mathrm{J}$. Chem. Phys. 1991, 95, 3924-3929.

[160] F. J. Lovas, H. Hartwig, The Microwave Spectrum of the Methanol Dimer for $K=0$ and 1 States, J. Mol. Spectrosc. 1997, 185, 98-109.

[161] S. Coussan, A. Loutellier, J. P. Perchard, S. Racine, A. Peremans, A. Tadjeddine, W. Q. Zheng, IR-induced interconversions between five conformers of methanol dimers trapped in nitrogen matrix, Chem. Phys. 1997, 223, 279-292.

[162] H.-L. Han, C. Camacho, H. A. Witek, Y.-P. Lee, Infrared absorption of methanol clusters $\left(\mathrm{CH}_{3} \mathrm{OH}\right)_{n}$ with $n=2-6$ recorded with a time-of-flight mass spectrometer using infrared depletion and vacuum-ultraviolet ionization, J. Chem. Phys. 2011, 134, 144309. 
[163] A. Bizzarri, S. Stolte, J. Reuss, J. C. G. M. van Duijneveldt-van de Rijdt, F. B. van Duijneveldt, Infrared Excitation and Dissociation of Methanol Dimers and Trimers, Chem. Phys. 1990, 143, 423-435.

[164] M. Heger, M. A. Suhm, R. A. Mata, Communication: Towards the binding energy and vibrational red shift of the simplest organic hydrogen bond: Harmonic constraints for methanol dimer, J. Chem. Phys. 2014, 141, 101105.

[165] M. Hippler, L. Oeltjen, M. Quack, High-Resolution Continuous-Wave-Diode Laser Cavity Ring-Down Spectroscopy of the Hydrogen Fluoride Dimer in a Pulsed Slit Jet Expansion: Two Components of the $N=2$ Triad near $1.3 \mu \mathrm{m}, J$. Phys. Chem. A 2007, 111, 12659-12668.

[166] R. Jiang, E. L. Sibert III, Surface hopping simulation of vibrational predissociation of methanol dimer, J. Chem. Phys. 2012, 136, 224104.

[167] H. Schaal, T. Häber, M. A. Suhm, Hydrogen Bonding in 2-Propanol. The Effect of Fluorination, J. Phys. Chem. A 2000, 104, 265-274.

[168] F. Kollipost, R. Wugt Larsen, A. V. Domanskaya, M. A. Suhm, Communication: The highest frequency hydrogen bond vibration and an experimental value for the dissociation energy of formic acid dimer, J. Chem. Phys. 2012, 136, 151101.

[169] P. J. Linstrom, W. G. Mallard (Hg.), NIST Chemistry WebBook, NIST Standard Reference Database Number 69, National Institute of Standards and Technology, Gaithersburg MD, 20899.

[170] R. Hildebrandt, V. Meyer, Technische Zeichnung: Montage beheizbarer Sättiger, Zeichenbüro, Institut für Physikalische Chemie der Universität Göttingen, 2013.

[171] R. Hildebrandt, V. Meyer, Technische Zeichnung: Paraboldüse $4 \times 0.15 \mathrm{~mm}$, Zeichenbüro, Institut für Physikalische Chemie der Universität Göttingen, 2013. 

Science is so much easier than real life.

J. M. B. 



\section{Lebenslauf}

\section{Persönliches}

Name: Katharina Otto

Geburtsdatum und -ort: 27.11.1984, Göttingen

Staatsangehörigkeit: deutsch

\section{Ausbildung}

1991-1994 Johann-Wolf Grundschule, Nörten-Hardenberg

1994-1997 Orientierungsstufe, Novalisschule Nörten-Hardenberg

1997-2004 Gymnasium Corvinianum Northeim

06/2004 Allgemeine Hochschulreife (Abitur)

Gymnasium Corvinianum Northeim

10/2005-11/2010 Diplomstudiengang Chemie

Georg-August-Universität Göttingen

10/2007 Vordiplom Chemie

Georg-August-Universität Göttingen

03/2009-08/2009 Auslandssemester im Bereich Organische Synthese

Universidade de Vigo (Prof. Dr. A. R. de Lera)

Vigo, Spanien

03/2010-09/2010 Diplomarbeit, Aminosäureester und ihre Aggregate

Institut für Physikalische Chemie (Prof. Dr. M. A. Suhm)

Georg-August-Universität Göttingen

11/2010 Diplom Chemie

Georg-August-Universität Göttingen

Seit 03/2011 Promotion Raman-Spektroskopie kleiner Moleküle und Molekülaggregate im Überschallstrahl nach thermischer Anregung Institut für Physikalische Chemie (Prof. Dr. M. A. Suhm) Georg-August-Universität Göttingen 